Florida International University

FIU Digital Commons

FIU Electronic Theses and Dissertations

University Graduate School

$11-13-2020$

\title{
Photo-production of Eta Mesons
}

Mahmoud Mohamed Kamel

Florida International University, mkame006@fiu.edu

Follow this and additional works at: https://digitalcommons.fiu.edu/etd

Part of the Nuclear Commons

\section{Recommended Citation}

Kamel, Mahmoud Mohamed, "Photo-production of Eta Mesons" (2020). FIU Electronic Theses and Dissertations. 4556.

https://digitalcommons.fiu.edu/etd/4556

This work is brought to you for free and open access by the University Graduate School at FIU Digital Commons. It has been accepted for inclusion in FIU Electronic Theses and Dissertations by an authorized administrator of FIU Digital Commons. For more information, please contact dcc@fiu.edu. 


\section{FLORIDA INTERNATIONAL UNIVERSITY}

Miami, Florida

\section{PHOTO-PRODUCTION OF $\eta$ MESONS}

A dissertation submitted in partial fulfillment of the requirements of the degree of DOCTOR OF PHILOSOPHY

in PHYSICS

by

Mahmoud Kamel 
To: Dean Michael R. Heithaus

College of Arts, Sciences and Education

This dissertation, written by Mahmoud Kamel, and entitled Photo-production of $\eta$ Mesons, having been approved in respect to style and intellectual content, is referred to you for judgment.

We have read this dissertation and recommend that it be approved.

Misak Sargsian

Joerg Reinhold

$\begin{array}{r}\text { Lei Guo } \\ \hline \text { Jose Allmirall }\end{array}$

Werner Boeglin, Major Professor

Date of Defense: November 13, 2020

The dissertation of Mahmoud Kamel is approved.

Dean Michael R. Heithaus

College of Arts, Sciences and Education

Andrés G. Gil

Vice President for Research and Economic Development and Dean of the University Graduate School

Florida International University, 2020 
(c) Copyright 2020 by Mahmoud Kamel All rights reserved. 


\section{DEDICATION}

To my mother Soad Shalaby, my wife Ashgan Aboutaleb, and my life long teacher and brother Ahmed Kamel 


\section{ACKNOWLEDGMENTS}

First and foremost, I'm very grateful to my major adviser Werner Boeglin. I cannot find enough words to thank him for all his time and effort through out this work. Without his guidance, support, and encouragement, this work would have never been possible to achieve. I appreciate my committee members Joerg Reinhold, Lei Guo, Misak Sargsian, and Jose Allmirall for their useful discussions and suggestions.

I would like to thank Jefferson lab staff and the GlueX members for the team work during the experiment and constructive comments through data taking, detector calibration, and analysis work. I would like to mention some names although there is not enough room to mention all of them. Mark Ito, Simon Taylor, and Beni Zihlmann were dedicated to help during the start counter work. Many thanks to Simon for providing me the theoretical predictions for the $\eta$ photo-production differential cross sections by J.M Laget. The private communications with Dave Mack, Justin Stevens and Sean Dobbs were very helpful during analysis.

Thanks to Volker Crede, who provided me with the CLAS2020 experimental data, I was able to provide thorough comparisons of the $\eta$ photo-production differential cross sections between The CLAS and the GlueX experiments.

I will never forget my colleagues at Florida International University and specifically the nuclear group friends. Among so many I remember useful conversations with Adam Freez, Rafael Badui, Jonathan Castellanos, Frank Vera, Christopher Leon, Carlos Yero, Rupesh Dotel, and Tolga Erbora. 


\title{
ABSTRACT OF THE DISSERTATION \\ PHOTO-PRODUCTION OF $\eta$ MESONS
}

\author{
by \\ Mahmoud Kamel \\ Florida International University, 2020 \\ Miami, Florida \\ Professor Werner Boeglin, Major Professor
}

In this work we present the GlueX experiment extensive high statistics measurements of the $\eta$ meson photo-production differential cross sections at beam energies between 3 $\mathrm{GeV}$ and 11.6 GeV. We compared the low energy $\left(2.9 \mathrm{GeV}<E_{\gamma}<5.9 \mathrm{GeV}\right)$ differential cross section $(d \sigma / d \Omega)$ results to previous experimental results from the Continuous Electron Beam Accelerator Facility (CEBAF) Large Acceptance Spectrometer (CLAS) collaboration. These $(d \sigma / d \Omega)$ results are also compared to the theoretical isobar model EtaMAID2018 by Tiator et. al., and the Regge model by Nys et. al. The differential cross section $d \sigma / d t$ is also presented and compared to the previous experimental results, and to the above models, in addition to a model by J. M. Laget. The high energy $\left(6.2 \mathrm{GeV}<E_{\gamma}<11.6 \mathrm{GeV}\right)$ cross section results $(d \sigma / d \Omega$, and $d \sigma / d t)$ are compared to the isobar model EtaMAID2018. These results will provide new constraints on $\eta$ photo-production mechanisms and high energy tchannel processes. 


\section{TABLE OF CONTENTS}

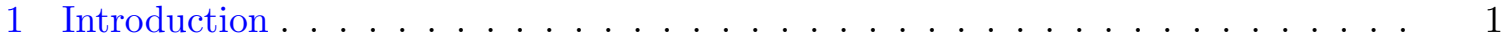

1.1 Dissertation Structure . . . . . . . . . . . . . . . . 2

1.2 Constituent Quark Model . . . . . . . . . . . . . . . . . . 3

1.3 Introduction to Scattering and Cross Section . . . . . . . . . . . 6

1.4 Two Body Kinematics and Regge Formalism . . . . . . . . . . . . . . . . 9

2 The GlueX Experiment . . . . . . . . . . . . . . . . . . . . . . . . 14

2.1 CEBAF Electron Beam . . . . . . . . . . . . . . . . . 14

2.2 Coherent bremsstrahlung . . . . . . . . . . . . . . . 15

2.3 The GlueX Beam Line and Tagger Hall . . . . . . . . . . . . . . . 17

2.4 The Gluex Detector Components . . . . . . . . . . . . . . . . . 20

2.5 Start Counter $(\mathrm{SC}) \ldots \ldots \ldots \ldots \ldots \ldots \ldots \ldots$

2.6 Central Drift Chamber . . . . . . . . . . . . . . . . . . . 33

2.7 Forward Drift Chamber . . . . . . . . . . . . . . . 34

2.8 Barrel Calorimeter . . . . . . . . . . . . . . . . . . 35

2.9 Time of Flight . . . . . . . . . . . . . . . . . . . . 37

2.10 Forward Calorimeter . . . . . . . . . . . . . . . . 37

3 Event Selection for $\eta$ Photo-production for $2.9 \mathrm{GeV} \leq E_{\gamma} \leq 5.9 \mathrm{GeV} \ldots \ldots$

3.1 Event Selection of $\eta$ Mesons . . . . . . . . . . . . . . . . . . 39

3.2 Data Monte-Carlo comparisons . . . . . . . . . . . . . . . . . 45

3.3 Differential Cross Section Determination . . . . . . . . . . . . . 51

4 Differential Cross Sections For $\eta$-Photo-production At Low Beam Energies 2.9 $\mathrm{GeV} \leq E_{\gamma} \leq 5.9 \mathrm{GeV} \ldots \ldots \ldots \ldots \ldots \ldots \ldots$

4.1 Differential Cross Section $(\mathbf{d} \sigma / \mathbf{d} \Omega)$ Results . . . . . . . . . . . . . . 57

4.2 Differential Cross Section $(\mathbf{d} \sigma / \mathbf{d t})$ Results . . . . . . . . . . . . . 60

4.3 Systematic Studies . . . . . . . . . . . . . . . . 73

5 Differential Cross Sections For $\eta$-Photo-production at High Beam Energies 6.2 $\mathrm{GeV} \leq E_{\gamma} \leq 11.6 \mathrm{GeV} \ldots \ldots \ldots \ldots \ldots \ldots$

5.1 Data and Monte-Carlo (MC) Comparisons . . . . . . . . . . . . 85

5.2 Raw Yield Extraction and Acceptance Calculations . . . . . . . . . . . . . 88

5.3 Differential Cross Section Results $(d \sigma / d \Omega)$ for the High Energy Data . . . . 95

5.4 Differential cross sections $(d \sigma / d t)$ for High Energy Data . . . . . . . . . . 102

5.5 Combined $d \sigma / d t$ discussion for $2.54<W<4.8 \mathrm{GeV} \ldots \ldots \ldots$. . . . . 107

6 Summary and Conclusion . . . . . . . . . . . . . . . . . . 111

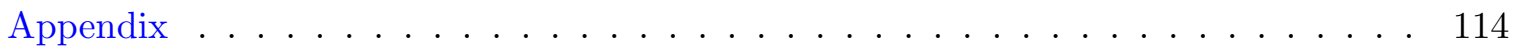

Bibliography . . . . . . . . . . . . . . . . . 127 


\section{LIST OF TABLES}

TABLE

PAGE

3.1 The value of constants used in equation $3.2 \ldots \ldots \ldots \ldots$

3.2 The central values of each of the center of mass energy bins and the corresponding beam energy. . . . . . . . . . . . . . . . 53

3.3 The beginning of the $\cos (\theta)$ bin and its width. . . . . . . . . . . . 54

5.1 The central values of each center of mass energy bins and the corresponding beam energy for the high energy data. . . . . . . . . . . . . 86

$1 \quad \eta$ differential cross section for center of mass energy $=2542 \mathrm{MeV} \ldots \ldots . .120$

$2 \quad \eta$ differential cross section for center of mass energy $=2580 \mathrm{MeV} \ldots \ldots . . .121$

$3 \quad \eta$ differential cross section for center of mass energy $=2620 \mathrm{MeV} \ldots \ldots . . \quad 121$

$4 \quad \eta$ differential cross section for center of mass energy $=2660 \mathrm{MeV} \ldots \ldots$

$5 \quad \eta$ differential cross section for center of mass energy $=2700 \mathrm{MeV} \ldots \ldots . . .122$

$6 \quad \eta$ differential cross section for center of mass energy $=2750 \mathrm{MeV} \ldots \ldots . .123$

$7 \quad \eta$ differential cross section for center of mass energy $=2810 \mathrm{MeV} \ldots \ldots$

$8 \quad \eta$ differential cross section for center of mass energy $=2875 \mathrm{MeV} \ldots \ldots . .124$

$9 \quad \eta$ differential cross section for center of mass energy $=2955 \mathrm{MeV} \ldots \ldots . .124$

$10 \eta$ differential cross section for center of mass energy $=3053 \mathrm{MeV} \ldots \ldots . .125$

$11 \eta$ differential cross section for center of mass energy $=3155 \mathrm{MeV} \ldots \ldots . .125$

$12 \eta$ differential cross section for center of mass energy $=3277 \mathrm{MeV} \ldots \ldots . .126$ 


\section{LIST OF FIGURES}

FIGURE

PAGE

1.1 Contributions of t-channel processes to $\eta$ photoproduction from (a) single poles, (b) Regge poles, and (c) Regge cuts with example of two Reggeions' rescattering of $(\rho, \omega)$ mseons, and (Pomeron, $\left.f_{2}\right)$ mesons. Modified from [10]

1.2 Center of mass energy $(\mathrm{W})$ as a function of the photon beam energy $E_{\gamma} \ldots$

1.3 (a) The incoming quarks from a pion beam have $L=0$, and $S=0$. The scattering process results in a hybrid mesons with non exotic quantum numbers. (b) In photoproduction the incoming quarks have $L=0$ and $S=1$, and therefore hybrid mesons with exotic quantum numbers are more probable than pion

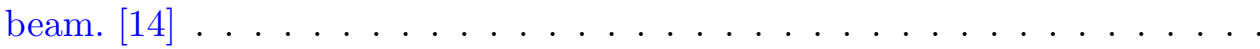

1.4 Pseudoscalar mesons with $\mathrm{L}=0$, and $\mathrm{S}=0$, and vector mesons with $L=0$, and

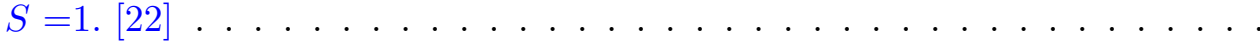

1.5 The minimum momentum transfer as a function of W. . . . . . . . . 11

1.6 $\cos \theta_{c m}^{\eta}$ as a function of $\mathrm{W}$ for three values of momentum transfer $(\mathrm{t}) . \quad \ldots$

1.7 Particle spins vs their squared masses t. Confirmed four degenerate Regge trajectories are shown. There is some doubt about the particles in the square bracket. The straight line is $\alpha(t)=0.5+0.9 t[27] \ldots \ldots \ldots$

2.1 Schematic diagram for accelerator site, the injector in green, Hall D and tagger hall are in yellow. Halls A, B, and $\mathrm{C}$ in the opposite side. . . . . . .

2.2 Uncollimated and collimated coherent bremsstrahlung spectrum, calculated for diamond radiator $15 \mu \mathrm{m}$ thick and a $1 \mu \mathrm{A}$ electron beam of $12 \mathrm{GeV}$ [35].

2.3 Tagger hall view, not to scale . . . . . . . . . . .

2.4 Photon flux versus energy as determined by PS (a). TPOL polarization determination as a function of energy (b). The polarization planes that are parallel and perpendicullar to the horizontal are denoted by PARA and PERP [37]. . . . . . . . . . . . . . . . . . .

2.5 Schematic diagram for the Gluex detector and beam line. The distance between the diamond wafer and the liquid hydrogen target is $70 \mathrm{~m}$ in reality. .

2.6 SC mounted the GlueX target. The beam travels down the central axis of the SC from right to left. During run operations the SC inserted in the bore of the central drift chamber shown at the top left corner. . . . . . . . . 
2.7 SC paddle geometry. Straight, bend, and nose sections . . . . . . . . .

2.8 Sketch to illustrate the $\mathrm{SC}$ propagation time . . . . . . . . . . .

2.9 Propagation time $(\mathrm{ns})$ vs $\mathrm{z}(\mathrm{cm})$. The red dotted line denotes the first 10 $\mathrm{cm}$ of the straight section. The length from the red dotted line to the first solid line denotes the rest of the straight section. The bend section is located between the red solid lines. The nose section starts right after the second solid red line. The magenta line is at the beginning of the last nose section interval.

2.10 Gaussian fits for SC propagation time peaks for four selected intervals in the nose section. . . . . . . . . . . . . . . . .

2.11 Propagation time $(\mathrm{ns}) \mathrm{vs} \mathrm{z}(\mathrm{cm})$. Three linear fitted regions . . . . . . .

2.12 $\mathrm{SC}$ propagation time (ns) vs $\mathrm{z}(\mathrm{cm})$. The main $\mathrm{RF}$ bunch band is aligned at about 0 ns after correction. . . . . . . . . . . . .

2.13 The total number of the SC recorded hit is determined within the two solid lines. The dashed line in both sides is the end of each side band intervals used for accidental calculations. . . . . . . . . . . . . . .

2.14 The overall SC efficiency (a). The Straight (brown), bend (violet), and nose (red) section efficiencies $(\mathrm{b}) \ldots \ldots \ldots \ldots \ldots$

2.15 The SC efficiency as a function $\mathrm{z}$ coordinate of the charged particle track intersection with the $\mathrm{SC}$ paddle. . . . . . . . . . . . .

2.16 Probability distribution for efficiency calculation for total number of events $\mathrm{N}=5$, and $\mathrm{N}=100 . \ldots \ldots \ldots \ldots \ldots$

2.17 Efficiency error estimation using the Baysian method for $99 \%$ efficiency of

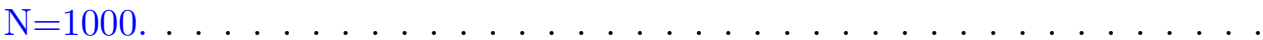

2.18 Predicted $\left(N_{p}\right)$ and recorded tracks $\left(N_{r}\right)$ to the SC. The accidental tracks $\left(N_{a}\right)$ and the difference $N_{r}-N_{a}$ are shown in the bottom plots. . . . . .

2.19 The SC Time resolution during Spring 2018 run period as a function of the run number. These runs started with $41 \mathrm{NNN}$, where $\mathrm{NNN}$ is the $\mathrm{x}$-axis number. 33

2.20 CDC stereo straw tubes. Partially completed layer of stereo straw tubes, during $\mathrm{CDC}$ construction, surrounding a layer of straw tubes at the opposite

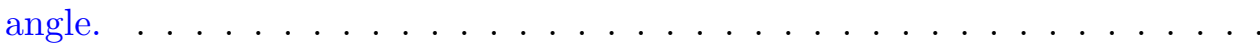

2.21 FDC cell view. Artistic view of one detector cell from left to right: upstream cathode, spacer ring, wire plane, downstream cathode, ground plane (black disk) glued to the back of the downstream cathode, end window (gray) only at the end of the package. . . . . . . . . . . . . 
2.22 Sketch of the Barrel Calorimeter and readout. (a) A three-dimensional BCAL view; (b) top-half cutaway (partial side view) of a BCAL module showing its polar angle coverage and location with respect to the GlueX target; (c) end view of the BCAL depicting all 48 azimuthal modules and (d) wedge-shaped end view of a single module. . . . . . . . . . . . . . . . . . . .

2.23 TOF wall on its support frame. . . . . . . . . . . . . . . . .

2.24 The energy resolution $\sigma_{\gamma} / E_{\gamma}$ for single photons in the a) BCAL and b)FCAL calculated from $\eta$ mass distribution with the assumption that only the energy resolution contribute to its width. Black solid circles are data, and red open squares are simulation.

3.1 Proton identification timing cut in the BCAL . . . . . . . . . . .

3.2 Time difference between tagged beam photons and RF time. The peak in between the red lines indicate the in-time beam photons. Outside these red lines show the out-of-time beam photons that will be used for accidental subtraction. . . . . . . . . . . . . . . . .

$3.3 \chi^{2}$ distributions scaling from kinematic fitting of data event (blue) and simulation events (red). A nominal cut is applied at $\chi^{2}=30 . \ldots \ldots$. . . .

3.4 Kinematic fitting confidence level for data (left), and simulation (right) after $\chi^{2}$ cut has been applied. . . . . . . . . . . . . . . . .

3.5 The production vertex $\mathrm{z}$ coordinate. The center of the target is located at $\mathrm{z}$ $=65 \mathrm{~cm}$ (dashed dotted blue). The selected events (between red lines) have their vertices within the target and are avoiding the target wall (magenta lines). . . . . . . . . . . . . . . . . .

$3.6\left|M_{M}\right|^{2}$ distribution with cut (red lines) . . . . . . . . . . .

3.7 The $2 \gamma$ invariant mass spectrum for events passing all the selection cuts. . .

$3.8 \eta$ polar angular distribution comparison between data (blue) and simulation (red) in the lab frame for all center of mass energy bins, $\mathrm{W}=2.542 \mathrm{GeV}$ (top left) up to $\mathrm{W}=3.277 \mathrm{GeV}$ (bottom right) . . . . . . . . . . . . .

3.9 Recoil proton angular distribution comparison between data (blue) and simulation (red) in the lab frame for $\mathrm{W}=2.542 \mathrm{GeV}$ (top left) up to $\mathrm{W}=3.277$ $\mathrm{GeV}$ (bottom right). . . . . . . . . . . . . . . . .

3.10 Recoil proton momentum distribution comparison between data (blue) and simulation (red) in the lab frame for $\mathrm{W}=2.542 \mathrm{GeV}$ (top left) up to $\mathrm{W}=$ $3.277 \mathrm{GeV}$ (bottom right). . . . . . . . . . . . . . . . 
$3.11 \eta$ momentum distribution comparison between data (blue) and simulation (red) in the lab frame. . . . . . . . . . . . . . . . . . . 50

$3.12 \gamma \gamma$ invariant mass distribution fit to extract the yield for several bins at the center of mass energy $\mathrm{W}=3.053 \mathrm{GeV} \ldots \ldots \ldots \ldots . \ldots \ldots$

3.13 Photon flux at each of the center of mass energy bin . . . . . . . . 54

3.14 Acceptance as a function of $\cos \left(\theta_{c m}^{\eta}\right)$ from $\mathrm{W}=2.542$ up to $2.750 \mathrm{GeV} \ldots . \quad 55$

3.15 Acceptance as a function of $\cos \left(\theta_{c m}^{\eta}\right)$ from $\mathrm{W}=2.810$ up to $3.277 \mathrm{GeV} \ldots . \quad 56$

4.1 Differential cross section results in both linear (left), and log scale (right) for $\mathrm{W}=2542,2580$, and $2620 \mathrm{MeV} \ldots \ldots \ldots \ldots \ldots \ldots$

4.2 Same as Fig. 4.1, but $\mathrm{W}=2660,2700$, and $2750 \mathrm{MeV} \ldots \ldots \ldots$. . . . 59

4.3 Same as Fig. 4.1, but $\mathrm{W}=2810,2875$, and $2955 \mathrm{MeV} \ldots \ldots \ldots$. . . . 61

4.4 Same as in Fig. 4.1, but for $\mathrm{W}=3.053$ up to $3.277 \mathrm{GeV} . \quad \ldots \ldots \ldots$. . . 62

4.5 Differential cross section of $\eta$ mesons in a linear scale as a function of -t. W $=2.542 \mathrm{GeV}$ up to $\mathrm{W}=2.750 \mathrm{GeV} \ldots \ldots \ldots \ldots$. . . . . . . 64

4.6 Same as 4.5 but for $\mathrm{W}=2.810 \mathrm{GeV}$ up to $\mathrm{W}=3.277 \mathrm{GeV} \ldots \ldots \ldots$

4.7 Weighted average differential cross section ratios between GlueX and EtaMAID2018 (blue), and CLAS2020 and EtaMAID2018 (black), as a function of $-\mathrm{t} . \mathrm{W}=2.542 \mathrm{GeV}$ up to $\mathrm{W}=2.750 \mathrm{GeV} \ldots \ldots \ldots$. . . . . . . . 66

4.8 Same as 4.7, but for $\mathrm{W}=2.810 \mathrm{GeV}$ up to $\mathrm{W}=3.277 \mathrm{GeV} \ldots \ldots \ldots$. . . . 67

4.9 Differential cross section of $\eta$ mesons in a log-scale as a function of - t. $\mathrm{W}=$ $2.542 \mathrm{GeV}$ up to $\mathrm{W}=2.750 \mathrm{GeV} \ldots \ldots \ldots \ldots$. . . . . . . . . 68

4.10 Same as Fig. 4.9, but for $\mathrm{W}=2.810 \mathrm{GeV}$ up to $\mathrm{W}=3.277 \mathrm{GeV}$. . . . . . 69

4.11 Differential cross section of $\eta$ mesons for $-\mathrm{t}<1.2 \mathrm{GeV}^{2}$ at $\mathrm{W}=2.542 \mathrm{GeV}$ up to $\mathrm{W}=2.750 \mathrm{GeV}$. The red line is the linear fit in the range of $0.14<-t<$

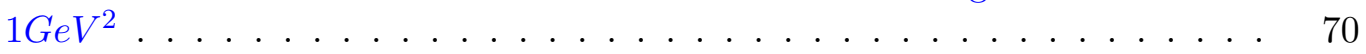

4.12 Same as Fig. 4.11, but for $\mathrm{W}=2.810 \mathrm{GeV}$ up to $\mathrm{W}=3.277 \mathrm{GeV}$. . . . . 72

4.13 Normalized differential cross section difference using genr8 events in addition to genEtaRegge. . . . . . . . . . . . . . . . . .

4.14 Normalized differential cross section difference using genr8 events in addition to genEtaRegge for $\mathrm{W}=2810 \mathrm{MeV}$ up to $3277 \mathrm{MeV} \ldots \ldots \ldots \ldots$ 
4.15 Normalized differential cross section difference for $\chi^{2}$ cuts. Magenta points are the cut of $\chi^{2}<20$, while the green points are $\chi^{2}<40 \ldots \ldots 77$

4.16 Same as 4.15 , but $\mathrm{W}=2.810 \mathrm{GeV}$ up to $\mathrm{W}=3.277 \mathrm{GeV} \ldots \ldots \ldots$

4.17 Normalized differential cross section differences for missing mass squared cuts. $\left|M_{M}^{2}\right|<0.020 \mathrm{GeV}^{2}$ in magenta, and $\left|M_{M}^{2}\right|<0.040 \mathrm{GeV}^{2}$ in green. . . 79

4.18 Same as 4.17 , but $\mathrm{W}=2.810 \mathrm{GeV}$ up to $\mathrm{W}=3.277 \mathrm{GeV} \ldots \ldots \ldots$

4.19 Normalized differential cross section differences for z-vertex cuts for $52<z<$ $78 \mathrm{~cm}$ in magenta, and for $50<z<80 \mathrm{~cm}$ in green. . . . . . . . . 81

4.20 Same as 4.19 , but $\mathrm{W}=2.810 \mathrm{GeV}$ up to $\mathrm{W}=3.277 \mathrm{GeV} \ldots \ldots$. . . . . 82

4.21 Normalized differential cross section differences for z-vertex cuts for proton momentum $<400 \mathrm{MeV}$ in green and for proton momentum $<300 \mathrm{MeV}$ in magenta. . . . . . . . . . . . . . . . .

4.22 Same as 4.19 , but $\mathrm{W}=2.810 \mathrm{GeV}$ up to $\mathrm{W}=3.277 \mathrm{GeV}$. . . . . . . .

5.1 Photon flux in 2017 data set f . . . . . . . . . . . . 86

$5.2\left|M_{\text {missing }}^{2}\right|$ distribution with cut (red line) $\ldots \ldots \ldots \ldots . \ldots . \ldots$

5.3 $\gamma \gamma$ mass distribution after applying all the event selection cuts. The red lines denote the mass interval over which the fit has been performed to extract

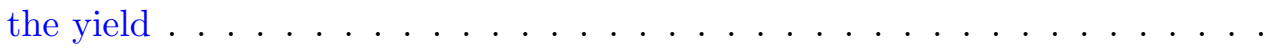

5.4 Momentum comparison of $\eta$ photo-production between data (blue) and simulation $($ red $)$ in the lab frame. . . . . . . . . . . . . .

5.5 Polar angle comparison of $\eta$ photo-production between data (blue) and simulation $($ red) in the lab frame. . . . . . . . . . . . . . . . . 90

5.6 Raw $\eta 2017$ yield for 6 center of mass energy bins 3.60, 3.71, 3.82, 3.93, 4.04, and $4.14 \mathrm{GeV}$. The inset shows zoom in yield for $\cos \theta_{c m}^{\eta}>0.6$ values. . . . .

5.7 Same as Fig. 5.6 but for $\mathrm{W}$ values of 4.24, 4.34, 4.44, 4.53, 4.62 and $4.71 \mathrm{GeV} .92$

5.8 GlueX detector acceptance for 6 center of mass energy bins 3.60, 3.71, 3.82, $3.93,4.04$, and $4.14 \mathrm{GeV} . \ldots \ldots \ldots . \ldots \ldots$

5.9 Same as 5.8 , but for $W=3.60,3.71,3.82,3.93,4.04$, and $4.14 \mathrm{GeV} \ldots \ldots 9$

5.10 Differential cross section $\left(\frac{d \sigma}{d \Omega}\right)$ for 6 center of mass energy bins 3.60, 3.71, 3.82, 3.93, 4.04, and 4.14 GeV. Gluex data(blue), EtaMAID2018, and Regge theoretical models are in grey and green lines, respectively. . . . . . . . . 
5.11 Same as 5.10 but for center of mass energy bins 4.24, 4.34, 4.44, 4.53, 4.62, and $4.71 \mathrm{GeV}$

5.12 Differential cross section $\left(\frac{d \sigma}{d \Omega}\right)$ for $\cos \theta_{c m}^{\eta}>0.6$ in a linear scale for 6 center of mass energy bins 3.60, 3.71, 3.82, 3.93, 4.04, and $4.14 \mathrm{GeV}$. Gluex data(blue), EtaMAID2018, and Regge theoretical models are in grey and green lines, respectively. . . . . . . . . . . . . . . . . .

5.13 Same as 5.12 but for center of mass energy bins 4.24, 4.34, 4.44, 4.53, 4.62, and $4.71 \mathrm{GeV} \ldots \ldots \ldots \ldots \ldots \ldots$

5.14 Weighted average differential cross section ratios between GlueX data and EtaMAID2018 model at $\cos \theta_{c m}^{\eta}>0.8$ for 6 center of mass energy bins 3.60, $3.71,3.82,3.93,4.04$, and $4.14 \mathrm{GeV} \ldots \ldots \ldots \ldots$

5.15 Same as Fig. 5.14. . . . . . . . . . . . . . . . . . . . . 101

5.16 Differential cross section $\left(\frac{d \sigma}{d t}\right)$ for 6 center of mass energy bins 3.60, 3.71, $3.82,3.93,4.04$, and $4.14 \mathrm{GeV}$ as a function of (-t). Gluex data(blue), EtaMAID2018 and Regge theoretical models are in grey and green lines, respectively. . . . . . . . . . . . . . . . . . .

5.17 Same as Fig. 5.16 but for 6 center of mass energy bins 4.24, 4.34, 4.44, 4.53, 4.62 , and $4.71 \mathrm{GeV}$.

5.18 Differential cross section $\left(\frac{d \sigma}{d t}\right)$ for 6 center of mass energy bins 3.60, 3.71, 3.82, $3.93,4.04$, and $4.14 \mathrm{GeV}$ as a function of (-t) for $-t<1.2 \mathrm{GeV}^{2}$. The red line is a linear fit for the GlueX data at $0.14<-t<1.0 \mathrm{GeV}^{2} \ldots \ldots$. . .

5.19 Same as Fig. 5.18 but for 6 center of mass energy bins 4.24, 4.34, 4.44, 4.53, 4.62 , and $4.71 \mathrm{GeV}$

5.20 Absolute value of the slopes and intercepts extracted from each energy bin

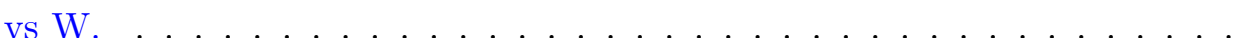

5.21 Combined total cross section in a linear (left), and log (right) scales from the low and high energy data sets Vs center of mass energy W.

5.22 The ratios of the extrapolated cross section (blue) and $\sigma_{t}$ (green) with respect to the IC. The yellow points are the $\log (s)$ values. . . . . . . . . . . .

5.23 The ratios of the extrapolated cross section (blue), $\sigma_{t}$ (green), and IC (magenta) with respect to the EtaMAID2018 total cross section. . . . . . . . 109

5.24 The values of $\alpha^{\prime}$ for all energy bins. The blue line is a linear fit of $0.50 \pm 0.01110$

$1 \pi^{+} \pi^{-} \gamma$ invariant mass before applying Dalitz cut. . . . . . . . . . . 115 
2 Invariant mass squared of $\pi^{-} \gamma \mathrm{vs} \pi^{+} \gamma$ for data (top) and simulation (bottom). 116

3 Invariant mass squared of $\pi^{-} \gamma \mathrm{vs} \pi^{+} \gamma$ for data (top) and simulation (bottom) after 2D Dalitz cut. . . . . . . . . . . . . . . . 117

$4 \quad \pi^{+} \pi^{-} \gamma$ invariant mass after applying Dalitz cut. . . . . . . . . . 118

5 (Top) $\pi^{+} \pi^{-} \gamma$ yield with linear (quadratic) background in red (magenta). The yield of $\pi^{+} \pi^{-} \eta$ is in blue. (bottom) Experimental branching ratio fit(yellow box) and comparison to the PDG value with its error (blue solid and dashed lines). . . . . . . . . . . . . . . . . . . . . . 119 


\section{CHAPTER 1}

\section{Introduction}

The primary goal of the GlueX experiment is to study the light meson spectrum and search for exotic and hybrid mesons. Studying the light meson spectrum and confirming the existence of exotic and hyprid mesons will provide a better understanding of the theory of the strong force, Quantum Chromo Dynamics (QCD), in the non perturbative regime. The production of the lightest multiplet of exotic mesons with $J^{P C}=1^{-+}$(defined in section 1.2) involves the same Regge exchanges that appear in production of ordinary pseudoscalar mesons like the $\eta$, and the $\eta^{\prime}$ [1]. The reaction $\gamma p \rightarrow \eta p$ leads to one of the simplest final states in the GlueX experiment to test the Regge theory for s (total energy), and t (momentum transfer) dependence (an exact definition is presented later in this chapter). The photo-production of $\eta / \eta^{\prime}$ mesons has been studied extensively in the resonance region near the threshold in the last two decades [2-7]. Pseudoscalar mesons like $\eta$ and $\eta^{\prime}$ have isospin $\mathrm{I}=0$, and therefore any final $\mathrm{N} \eta$ or $\mathrm{N} \eta^{\prime}$ state can only originate from intermediate nucleon states with $\mathrm{I}=1 / 2$. Thus they act as an isospin filter for nucleon resonance states. The $\eta$ photo-production near threshold is dominated by the nucleon resonance $S_{11}(1535)$. Other resonances like the $S_{11}(1650)$ make only minor contributions to the photo-production cross section near threshold.

The differential cross section for $\eta$ and $\eta^{\prime}$ photoproduction was measured up to a center of mass energy $\mathrm{W}=\sqrt{s}=3.16 \mathrm{GeV}$ by the CLAS collaboration $[8,9]$. For this intermediate energy range $\eta$ photoproduction contains s-channel contributions from other resonances as well as strong t-channel contributions in the forward direction. Several t-channel exchange mechanisms have been theoretically considered in the $\eta$ mesons photoproduction process such as a single pole, multiple Regge poles, and Regge cut exchanges [10], as shown in Fig. 1.1. A Regge cut can be understood as a production of $\eta$ meson via a vector or axial vector exchange at high energy, followed by a re-scattering through a pomeron or tensor exchange 1.1(c). A more detailed presentation of the Regge terminology will be discussed in section 1.4. Understanding meson photo-production mechanisms at photon energies $E_{\gamma}>4 \mathrm{GeV}$ 
$(\mathrm{W}>2.9 \mathrm{GeV})$ is essential for the search for exotic mesons in the GlueX program. The relationship between the photon beam energy $E_{\gamma}$ and the center of mass energy $(\mathrm{W})$ is derived in section 1.4, and shown in Fig. 1.2.

\section{t-channel processes to $\eta$ photo-production}
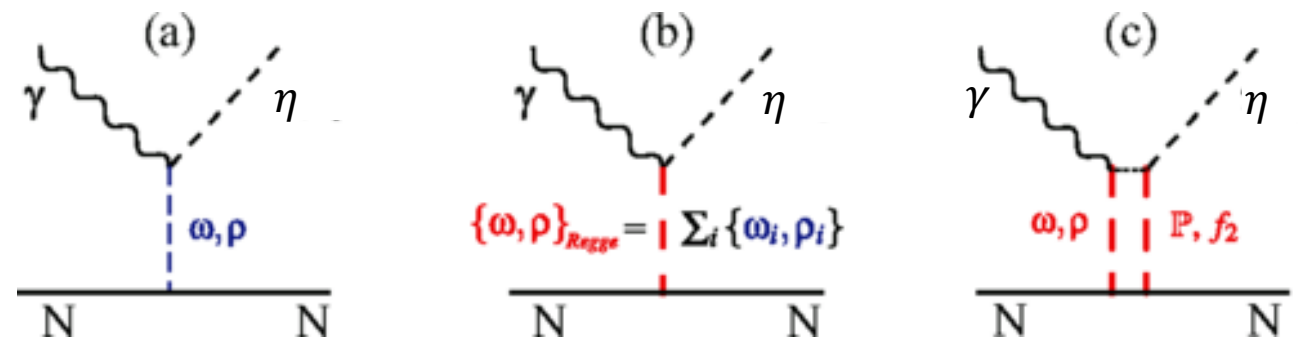

Figure 1.1: Contributions of t-channel processes to $\eta$ photoproduction from (a) single poles, (b) Regge poles, and (c) Regge cuts with example of two Reggeions' rescattering of $(\rho, \omega)$ mseons, and (Pomeron, $f_{2}$ ) mesons. Modified from [10]

In my thesis $\eta$ differential cross sections have been determined and compared to previous experimental results as well as several theoretical model predictions. One of these models is the new isobar model EtaMAID2018 [11]. In the new model $\eta$ photo-production has contributions from $21 \mathrm{~N}^{*}$ nucleon resonance states, and a background that is modeled using a Regge-cut. EtaMAID2018 model provides differential cross section predictions for both $\eta$, and $\eta^{\prime}$ photo-production off the proton up to $5 \mathrm{GeV}$ center of mass energy (W). The $\eta$ cross sections will be presented for photon energies $2.9 \mathrm{GeV} \leq E_{\gamma} \leq 5.9 \mathrm{GeV}$, that overlap with the existing data, and new data for a beam energy range of $6.2 \mathrm{GeV}$ to $11.6 \mathrm{GeV}$. The combined beam energy range of $2.9 \mathrm{GeV} \leq E_{\gamma} \leq 11.6 \mathrm{GeV}$ from the GlueX experiment will permit a comparison between previously measured cross sections and model predictions over a broad range of center of mass energies (W) between $2.54 \mathrm{GeV}$, and $4.71 \mathrm{GeV}$.

\subsection{Dissertation Structure}

A brief explanation of mesons multiplets, their composition, and nomenclature will be explained within the framework of the constituent quark model in the next section. The rest of chapter 1 contains an introduction to scattering and cross section as well as two body kinematics and Regge formalism. A detailed description of the GlueX experiment 
$\mathbf{W}$ vs $\mathbf{E}_{\gamma}$

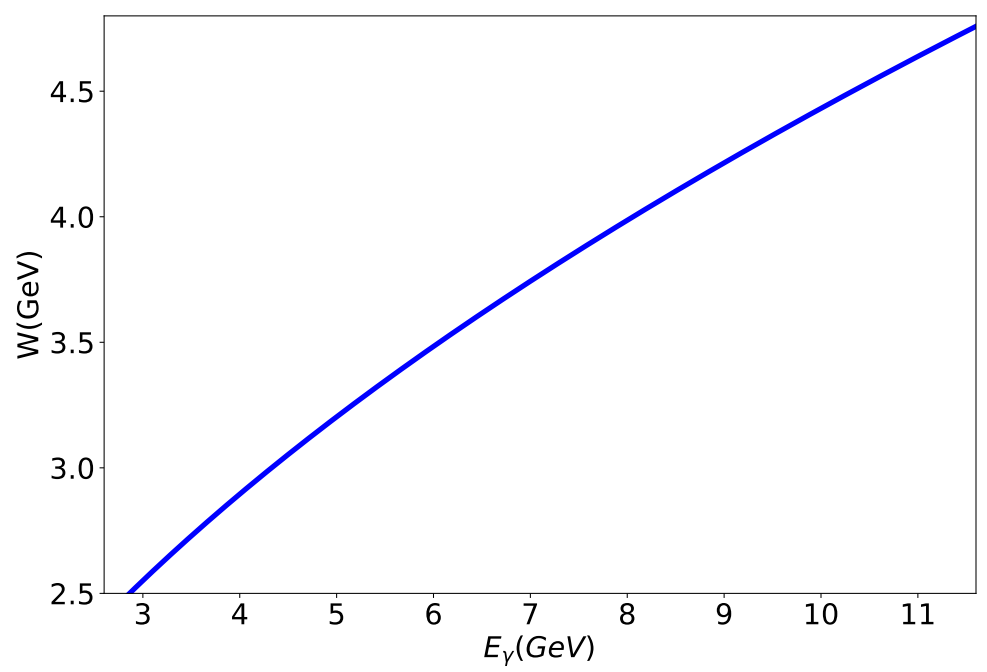

Figure 1.2: Center of mass energy $(\mathrm{W})$ as a function of the photon beam energy $E_{\gamma}$.

including its detector is presented in Chapter 2. Data selection for $\eta$ photo-production at low energy as well as differential cross section determination steps are described in Chapter 3. Differential cross sections from the low energy data set are shown in Chapter 4. Photoproduction differential cross section results of $\eta$ mesons at the nominal GlueX photon energy are reported in Chapter 5. A summary and conclusions are reported in Chapter 6. A short summary of $\eta^{\prime}$ analysis will be presented in Appendix A.1. Differential cross sections from the low energy data are tabulated in Appendix A.2.

\subsection{Constituent Quark Model}

In the constituent quark model a meson is described as a bound state of a quark and an antiquark $q \bar{q}[12]$. Mesons are charcterized by a unique set of quantum numbers $J^{P C}$, where $J$ is the total angular momentum, and $P$, and $C$ are parity and charge conjugation quantum numbers, respectively [13]. For any fermion anti-fermion system with total spin

$\vec{S}$, and relative angular momentum $\vec{L}$, the allowed $J^{P C}$ quantum number contributions are determined by the following rules: 


$$
\vec{J}=\vec{L}+\vec{S}, P=(-1)^{L+1} \text {, and } C=(-1)^{L+S} .
$$

\section{Photo-production vs $\pi$ probe}

(a)

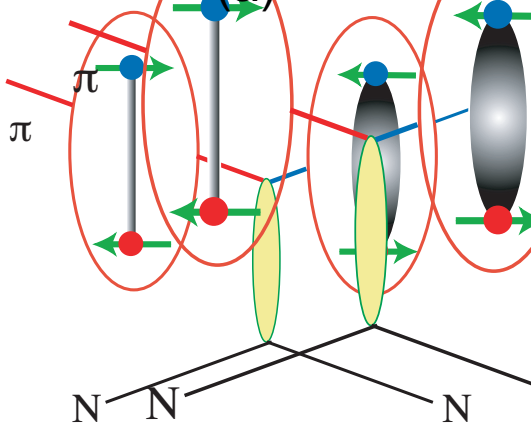

(b)

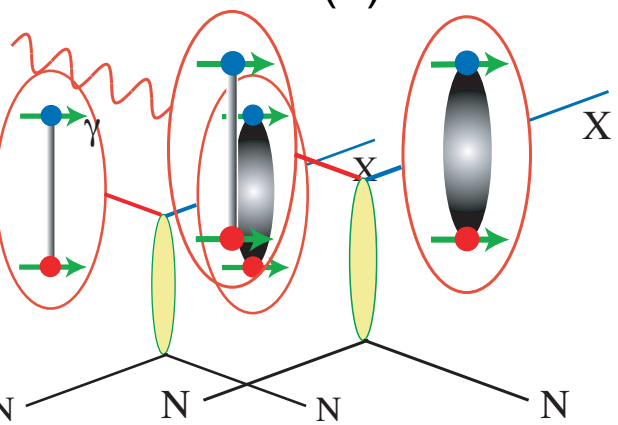

Figure 1.3: (a) The incoming quarks from a pion beam have $L=0$, and $S=0$. The scattering process results in a hybrid mesons with non exotic quantum numbers. (b) In photoproduction the incoming quarks have $L=0$ and $S=1$, and therefore hybrid mesons with exotic quantum numbers are more probable than pion beam. [14]

Exotic mesons can have $J^{P C}$ quantum numbers that do not follow these rules. Exotic mesons can be multiquark states $q \bar{q} q \bar{q}$, states containing only gluons (glueballs) or hybrid states [12]. Hybrid mesons are $q \bar{q} g$ states where the gluon (g) explicitly contributes its own degree of freedom. Hybrid mesons can have regular or exotic quantum numbers. Direct production of glueballs in photoproduction is supposed to be suppressed [15]. However, some models suggest that decays of hybrid mesons via a glueball component of lighter mesons may be enhanced [15].

The first reported evidence of an exotic meson came in 1988 from the GAMS collaboration at CERN using $\pi^{-} p \rightarrow \eta \pi^{0} n$ reaction data [13]. In 2010, the E852 colaboration published evidence for a $J^{P C}=1^{-+}$exotic meson decaying into $\eta^{\prime} \pi^{-}[12,13,16,17]$. More recently, the COMPASS experiment also observed an exotic signal and reported on the potentially exotic state $\pi_{1}(1600)$ [18]. Lattice Quantum Chromodynamics (LQCD) calculations predict many mesons with exotic quantum numbers such as $0^{--}, 0^{+-}, 1^{-+}$, ...etc [19]. A review of the theoretical and experimental status of hybrid hadrons can be found in [20]

Photo-production is expected to provide clear evidence for the existence of exotic mesons especially $1^{-+}$candidates as a result of the spin of the photon is the same as that of the exotic state. The flux tube model predicts that exotic hybrids are enhanced in 
photo-production (Fig. 1.3), while they are supressed in pion production reactions [14,21].
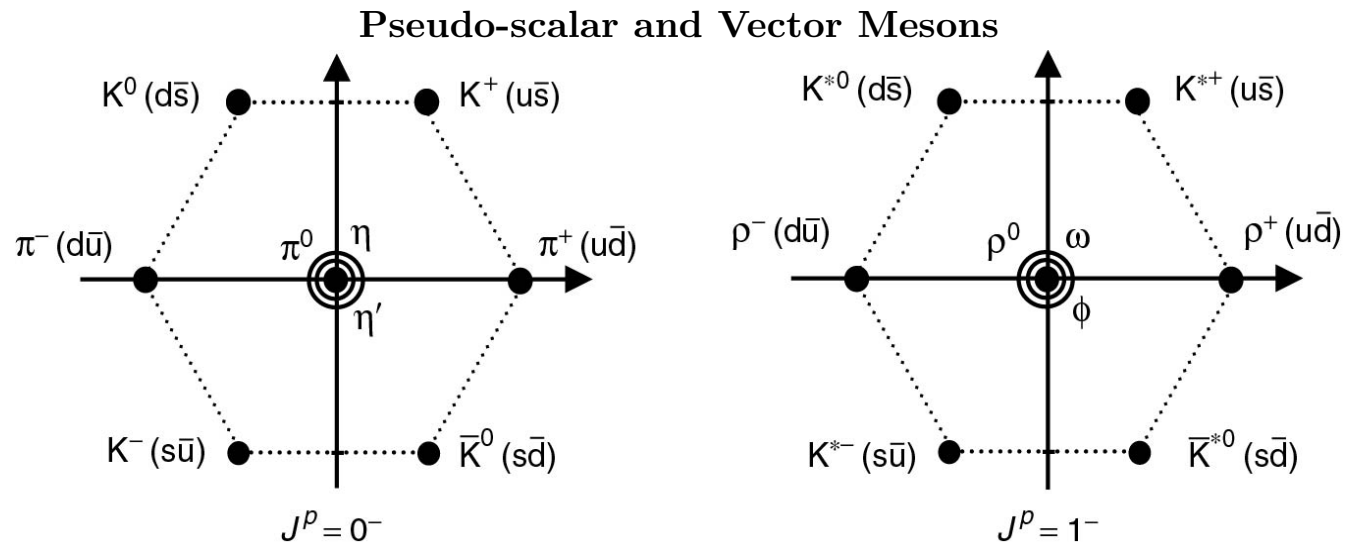

Figure 1.4: Pseudoscalar mesons with $\mathrm{L}=0$, and $\mathrm{S}=0$, and vector mesons with $L=0$, and $S$ $=1$. $[22]$

Following the SU(3) flavor symmetry, the $q \bar{q}$ flavor states are decomposed into octet and a singlet states. Figure 1.4 shows the lightest meson states $(\mathrm{L}=0)$ plotted in terms of the third component of isospin $I_{3}$ and the hypercharge Y. The isospin is analogous to the spin in its $\mathrm{SU}(2)$ symmetry, where the isospin doublet is formed of the up (u) and down (d) quark. Similarly the anti-up $(\bar{u})$ and anti-down $(\bar{d})$ quarks follow the same $\mathrm{SU}(2)$ symmetry as the quarks. The $\mathrm{SU}(3)$ flavor symmetry is an extension of $\mathrm{SU}(2)$ by adding the strange quark (s). $I_{3}$ and $\mathrm{Y}$ are given by

$$
I_{3}=\frac{1}{2}\left(n_{u}-n_{d}\right)
$$

where $n_{u}\left(n_{d}\right)$ is the total number of up(down) quark in the state

$$
Y=\frac{1}{3}\left(n_{u}+n_{d}-n_{s}\right)
$$

where $n_{s}$ is the total number of $\mathrm{s}$ quark in the state.

The $\pi^{0}$, and $\eta$ can be associated with the two $I_{3}=Y=0$ octet states. The $\eta^{\prime}$ can be considered as the $I_{3}=Y=0$ singlet state [22] as it has the largest mass of the three particles $(0.958 \mathrm{GeV})$. If the $\mathrm{SU}(3)$ flavor symmetry were exact, the two $I_{3}=Y=0$ octet states 
would have the exact same mass [22]. Since the mass of the strange quark $\left(m_{s} \sim 0.5 G e V\right)$ is much larger than the mass of the up and down quarks $\left(m_{u / d} \sim 0.3 \mathrm{GeV}\right)$, the $\mathrm{SU}(3)$ flavor symmetry is only approximate [22]. Therefore the physical isoscalar mesons $\left(\eta, \eta^{\prime}\right)$ are mixtures of the underlying $\mathrm{SU}(3)$ singlet " $\psi_{1}$ " and octet " $\psi_{8}$ " flavor wave functions, where $\psi_{1}$ is given by

$$
\psi_{1}=\frac{1}{\sqrt{3}}(u \bar{u}+d \bar{d}+s \bar{s})
$$

and $\psi_{8}$ is given by

$$
\psi_{8}=\frac{1}{\sqrt{6}}(u \bar{u}+d \bar{d}-2 s \bar{s})
$$

The physical $\eta^{\prime}$ meson wave function can be written as $\eta^{\prime}=\psi_{8} \sin \theta+\psi_{1} \cos \theta$, where $\theta$ is the mixing angle [23]. The wave function of the physical $\eta$, which has a mass of 0.547 GeV, can be written as $\eta=\psi_{8} \cos \theta-\psi_{1} \sin \theta . \eta$ and $\eta^{\prime}$ could also mix with other states, in particular with a pseudoscalar glueball [24], which led to speculations that the $\eta^{\prime}$ (and to a lesser extent the $\eta$ ) may contain a large fraction of glue [24]. Therefore measuring the differential cross section of $\eta$ and $\eta^{\prime}$ is a necessary step for understanding the production mechanism of both mesons.

\subsection{Introduction to Scattering and Cross Section}

In quantum mechanics, a beam of particles traveling in the z-direction and incident on a fixed target (scattering center), can be represented by a plane wave traveling in the zdirection with an amplitude of 1 as defined in equation 1.5

$$
\psi_{i}=e^{i k z}
$$

where $k=2 \pi / \lambda$, and $\lambda$ is the de Broglie wavelength, and the time dependence term $e^{-i \omega t}$ is omitted for simplicity. Any plane wave can be described by a superposition of spherical incoming and outgoing waves. At a radial distance $r$ from the scattering center the expansion 
of the plane wave when $\mathrm{kr} \gg 1$ is given by 1.6

$$
\psi_{i}=\frac{i}{2 k r} \sum_{l}(2 l+1)\left[(-1)^{l} e^{-i k r}-e^{i k r}\right] P_{l}(\cos \theta)
$$

where $P_{l}(\cos \theta)$ are the Legendre polynomials, $(-1)^{l} e^{-i k r}$ is an incoming wave, and $e^{i k r}$ is an outgoing wave. The scattering center can not change the incoming wave, but it will change the phase and amplitude of the outgoing wave. Therefore the total wave has the form:

$$
\psi_{\text {total }}=\frac{i}{2 k r} \sum_{l}(2 l+1)\left[(-1)^{l} e^{-i k r}-\eta_{l} e^{2 i \delta_{l}} e^{i k r}\right] P_{l}(\cos \theta)
$$

where

- $\eta_{l}$ is the amplitude of the $l$ th partial wave $\left(0<\eta_{l}<1\right)$.

- $2 \delta_{l}$ is the phase shift of the $l$ th partial wave.

The scattered wave $\left(\psi_{\text {scat }}\right)$ is given by the difference $\psi_{\text {total }}-\psi_{i}$.

$$
\psi_{\text {scat }}=\frac{e^{i k r}}{r} F(\theta)
$$

where $F(\theta)$ is the scattering amplitude, which is given by

$$
F(\theta)=\frac{1}{k} \sum_{l}(2 l+1)\left(\frac{\eta_{l} e^{2 i \delta_{l}}-1}{2 i}\right) P_{l}(\cos \theta)
$$

Equation 1.9 is an elastic scattering case since the wave number $k$ is the same before and after scattering. In the center of mass frame, $k$ and $\lambda$ represent the wave of the incident and target particles, and thus will not change in an elastic collision. The outgoing scattered flux in a solid angle $d \Omega$ in a sphere of radius $\mathrm{r}$ is given by

$$
v_{0} \psi_{\text {scat }} \psi_{\text {scat }}^{*} r^{2} d \Omega=v_{0}|F(\theta)|^{2} d \Omega
$$

where $v_{0}$ is the relative velocity of the outgoing particles with respect to the scattering center. The scattered flux is also defined as the product of the incident flux $\left(v_{i} \psi_{\text {scat }} \psi_{\text {scat }}^{*}\right)$ and the scattering cross section. Therefore for elastic scattering where $v_{i}=v_{0}$, one can 
write

$$
v_{0} d \sigma=v_{0}|F(\theta)|^{2} d \Omega
$$

Therefore,

$$
\left(\frac{d \sigma}{d \Omega}\right)_{\text {elastic }}=|F(\theta)|^{2}
$$

The total elastic cross section can be obtained by integrating over all angles to get

$$
\sigma_{e l}=4 \pi \lambda^{2} \sum_{l}(2 l+1)\left|\frac{\eta_{l} e^{2 i \delta_{l}}-1}{2 i}\right|^{2}
$$

The reaction cross section $\sigma_{r}$ is obtained from the conservation of probability (unitarity):

$$
\sigma_{r}=\int\left(\left|\psi_{\text {in }}\right|^{2}-\left|\psi_{\text {out }}\right|^{2}\right) r^{2} d \Omega
$$

where $\psi_{i n}$ is the first term in equation 1.6, while $\psi_{\text {out }}$ is the second term in equation 1.7. Therefore, the reaction cross section can be written as

$$
\sigma_{r}=\pi \lambda^{2} \sum_{l}(2 l+1)\left(1-\left|\eta_{l}\right|^{2}\right)
$$

The total cross section $\sigma_{\text {total }}$ is the sum of the elastic cross section and the reaction cross section, which takes the form:

$$
\sigma_{\text {total }}=\pi \lambda^{2} \sum_{l}(2 l+1) 2\left(1-\eta_{l} \cos 2 \delta_{l}\right)
$$

For the polar angle $\theta=0, P_{l}(1)=1$ for all $l$, and equation 1.9 gives the following

$$
\operatorname{Im} F(0)=\frac{1}{2 k} \sum_{l}(2 l+1)\left(1-\eta_{l} \cos 2 \delta_{l}\right)
$$

By comparing the last two equations, the optical theorem is obtained that relates the imaginary part of the forward elastic scattering amplitude to the total cross section.

$$
\operatorname{Im} F(0)=\frac{k}{4 \pi} \sigma_{\text {total }}
$$


Using experimental differential cross section results, one will be able to determine the $d \sigma / d t$ at $t=t_{\text {min }}$, by fitting a straight line to the log of the differential cross section $\left(\frac{d \sigma}{d t}\right)$ vs $(-\mathrm{t})$ over a small range of (-t) from 0.14 to $1.0 \mathrm{GeV}^{2}$, then extrapolate this line to $t=t_{\text {min }}$. The quantity $d \sigma / d t$ at $t=t_{\text {min }}$ is proportional to the total cross section as shown before. Differential cross sections of pseudoscalar mesons at high energy, $E_{\gamma}>4 \mathrm{GeV}$, show a forawrd peak at low (-t), $-t<2 \mathrm{GeV}^{2}$ and a backward peak at $u<1 \mathrm{GeV}^{2}$ [25]. The forward peak is caused by mesons exchange, while the backward peak is caused by baryons exchange [25]. Regge theory provides explainations in terms of energy and angluar dependence as $S^{2 \alpha(t)-2}$ in the forward peak, and $S^{2 \alpha(u)-2}$ in the backward peak, where $\alpha(t)$, and $\alpha(u)$ are the so-called Regge trajectories [25].

\subsection{Two Body Kinematics and Regge Formalism}

Kinematics of $1+2 \rightarrow 3+4$, where the particle $i=1, \ldots, 4$ has a mass $m_{i}$, and fourmomentum $P_{i}=\left(E_{i}, \mathbf{P}_{i}\right)$ are presented. The Lorentz-invariant Mandelstam variables $s, t$, and $u$ are defined as:

$$
\begin{aligned}
& s=\left(P_{1}+P_{2}\right)^{2} \\
& t=\left(P_{1}-P_{3}\right)^{2} \\
& u=\left(P_{1}-P_{4}\right)^{2}
\end{aligned}
$$

In the center of mass frame of the initial particles $(1,2)$, the four momenta are given by $P_{1}=\left(E_{1}, \mathbf{P}_{1}\right), P_{2}=\left(E_{2},-\mathbf{P}_{1}\right), P_{3}=\left(E_{3}, \mathbf{P}_{3}\right)$, and $P_{4}=\left(E_{4},-\mathbf{P}_{3}\right)$, where $\mathbf{P}_{1}$, and $\mathbf{P}_{3}$ are

the 3 momenta for particles 1 , and 3 in the center of mass frame. Therefore equation 1.20 can be written as:

$$
t=m_{1}^{2}+m_{3}^{2}-2\left(E_{1} E_{3}-\left|\mathbf{P}_{1}\right|\left|\mathbf{P}_{3}\right| \cos \theta\right)
$$

where $\theta$ is the angle between the three-momenta of particles 1 , and 3 . In case of photoproduction of $\eta$ mesons off the proton target, $s$, and $t$ are given by

$$
t=m_{\eta}^{2}-2\left(E_{\gamma} E_{\eta}-\left|\mathbf{P}_{\gamma}\right|\left|\mathbf{P}_{\eta}\right| \cos \theta_{c m}^{\eta}\right)
$$




$$
s=m_{p}^{2}+2 E_{\gamma} m_{p}
$$

where $m_{p}=0.938 G e V$ is the proton mass.

At fixed center of mass energy $\mathrm{W}=\sqrt{s}$, one can write

$$
t=a \cos \theta_{c m}^{\eta}+b
$$

where $a=2\left|\mathbf{P}_{\gamma}\right|\left|\mathbf{P}_{\eta}\right|$, and $b=m_{\eta}^{2}-2 E_{\gamma} E_{\eta}$. The relationship between the Mandelstam variable $\mathrm{t}$ and $\cos \theta_{c m}^{\eta}$ can also be witten as [26],

$$
t=\frac{\cos \theta_{c m}^{\eta}}{2 W^{2}} \lambda^{\frac{1}{2}}\left(s, 0, m_{p}^{2}\right) \lambda^{\frac{1}{2}}\left(s, m_{\eta}^{2}, m_{p}^{2}\right)+m_{\eta}^{2}-\frac{1}{2 W^{2}}\left(s-m_{p}^{2}\right)\left(s+m_{\eta}^{2}-m_{p}^{2}\right)
$$

where the function $\lambda(x, y, z)$ is given by

$$
\lambda(x, y, z)=(x-y-z)^{2}-4 y z
$$

For a given $\mathrm{W}$ value, one can write

$$
\frac{d \cos \theta_{c m}^{\eta}}{d t}=\frac{2 W^{2}}{\lambda^{\frac{1}{2}}\left(s, 0, m_{p}^{2}\right) \lambda^{\frac{1}{2}}\left(s, m_{\eta}^{2}, m_{p}^{2}\right)}
$$

Therefore for any differential cross section value $\frac{d \sigma}{d \Omega}$, one can obtain the differential cross section $d \sigma / d t$ as follows:

$$
\begin{aligned}
\frac{d \sigma}{d t} & =\frac{d \sigma}{d \Omega} \frac{d \Omega}{d t} \\
& =\frac{d \sigma}{d \Omega} 2 \pi \frac{d \cos \theta_{c m}^{\eta}}{d t}
\end{aligned}
$$

The differential cross section $\left(\frac{d \sigma}{d t}\right)$ is more relevant for $\eta$ photo-production at $E_{\gamma}>4 \mathrm{GeV}$, where the production mechanisms especially in the forward direction is a t-channel process. As $\mathrm{W}$ increases the minimum momentum transfer $\left(t_{\min }\right)$ is going to zero, see Fig. 1.5. The relationship between $\mathrm{W}$ and $\cos \theta_{c m}^{\eta}$ is shown in Fig. 1.6 for several momentum transfer 
$t_{\text {min }}$ vs $\mathbf{W}$

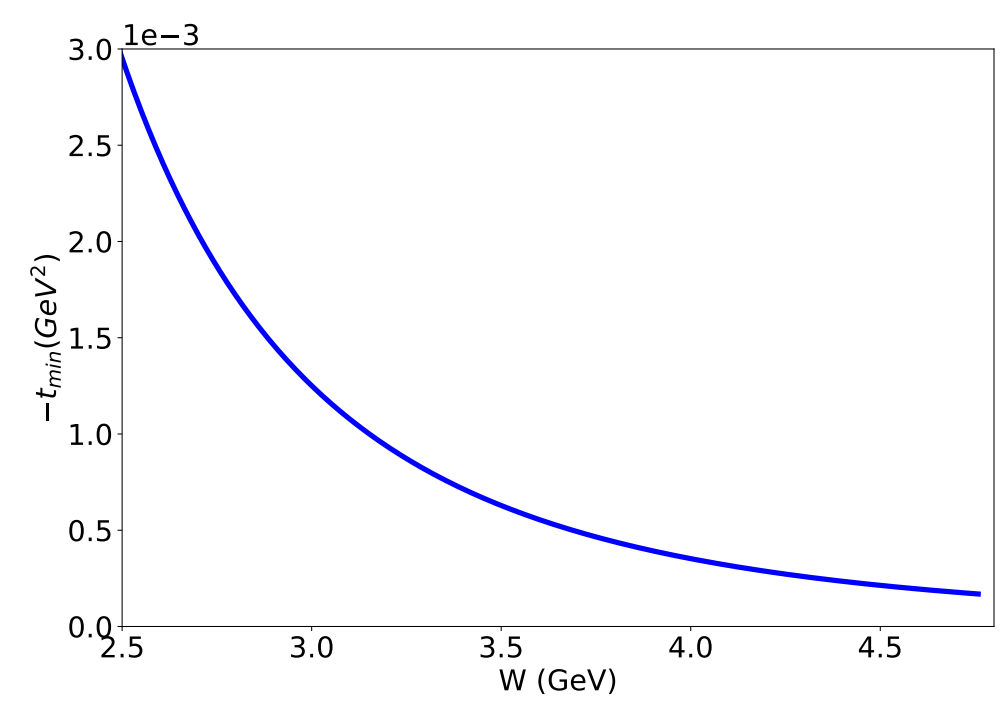

Figure 1.5: The minimum momentum transfer as a function of $\mathrm{W}$.

(t) values. As $|t|$ decreases, the production polar angle $\theta_{c m}^{\eta}$ decreases, and becomes more forward as $\mathrm{W}$ increases.

The Mandelstam variables are related by the equation 1.31

$$
s+t+u=\sum_{i=1}^{4} m_{i}^{2}
$$

Equation 1.31 indicates that a two body amplitude can only be a function of two independent variables. In this thesis as well as Regge theory one takes the amplitude as a function of s, and $\mathrm{t}[\mathrm{A}(\mathrm{s}, \mathrm{t})]$.

\subsubsection{Regge Formalism}

Regge formalism uses continuous complex anglular momentum values $(\ell)$, instead of the non-negative integers values $(l)$ [27]. A partial wave amplitude $A_{l}(t)$ can be expressed a functions $A(\ell, t)$ of complex $\ell$, such that

$$
A(\ell, t)=A_{l}(t), \quad l=0,1,2, \ldots
$$




\section{$\cos \theta_{\mathbf{c m}}^{\eta} \mathbf{v s} \mathbf{W}$}

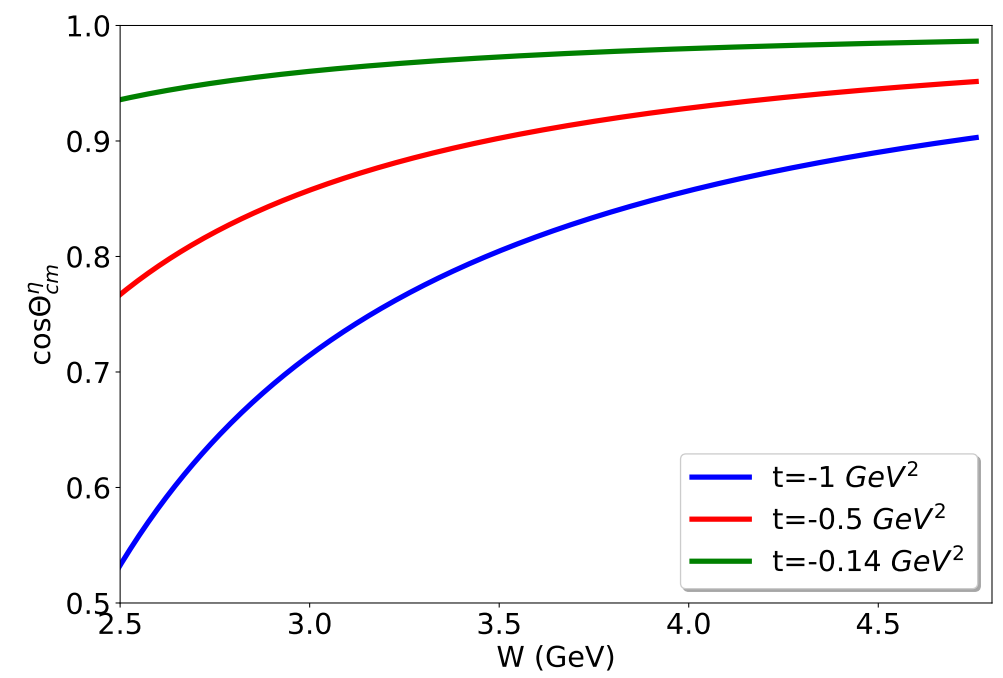

Figure 1.6: $\cos \theta_{c m}^{\eta}$ as a function of $\mathrm{W}$ for three values of momentum transfer $(\mathrm{t})$.

Certain assumptions are usually made for the complex amplitudes:

- Any singularity has a dynamic origin.

- Bound states are associated with poles.

- Thresholds are associated with cuts.

Regge found that the singularities of $A(\ell, t)$ in the complex $\ell$ plane are poles whose locations vary with $\mathrm{t}$ as $\ell=\alpha(t)$. These poles are called Regge poles, or reggeons (1.1a). Regge trajectories are the functions $\alpha(t)$. These Regge trajectories are associated with the exchange of families of particles. If $\alpha(t)$ is a non negative integer, values of $t$ correspond to the squared mass of a bound state or resonance having that spin $l$ as shown in Fig. 1.7.

The linear Regge trajectory, in general, can be written as

$$
\alpha(t)=\alpha_{0}+\alpha^{\prime} t
$$

where the pole in the lth partial wave takes the form

$$
A_{l}(t)=\frac{\beta(t)}{l-\alpha(t)}=\frac{\beta t}{\alpha^{\prime}\left(m_{l}^{2}-t\right)}
$$




\section{Regge trajectories}

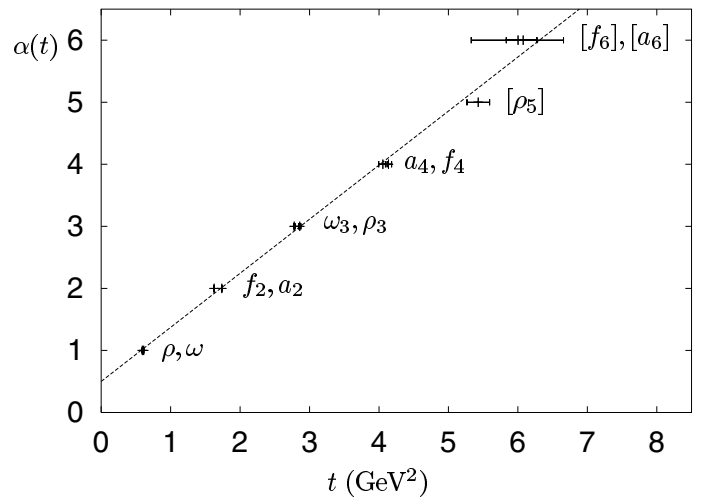

Figure 1.7: Particle spins vs their squared masses t. Confirmed four degenerate Regge trajectories are shown. There is some doubt about the particles in the square bracket. The straight line is $\alpha(t)=0.5+0.9 t[27]$.

Equation 1.34 indicates that there is a pole at $l=\alpha(t)$. The variable $\beta(t)$ is the residue function that represent the coupling of the pole to external particles. In a two body interaction, the differential cross section $\left(\frac{d \sigma}{d t}\right)$ predicted by Regge theory is proportional to $\left(\frac{s}{s_{0}}\right)^{2 \alpha(t)-2}$, where $S_{0}$ is the hadron mass scale $\left(1 \mathrm{GeV}^{2}\right)$, and $\alpha(t)$ is the leading Regge trajectory which can be exchanged.

$$
\frac{d \sigma}{d t} \propto\left(\frac{s}{s_{0}}\right)^{2\left(\alpha^{\prime} t+\alpha_{0}\right)-2}
$$

which implies

$$
\begin{gathered}
\log \left(\frac{d \sigma}{d t}\right) \propto C_{1}\left(\alpha^{\prime} t+\alpha_{0}-1\right] \\
\log \left(\frac{d \sigma}{d t}\right) \propto C_{0}+C_{1} \alpha^{\prime} t
\end{gathered}
$$

where $C_{1}=2 \log \left(\frac{s}{s_{0}}\right)$, and $C_{0}=C_{1}\left(\alpha_{0}-1\right)$. In the forward peak, one can write the expression

$$
\frac{d \sigma}{d t} \propto A \sigma_{t o t a l} e^{-\beta t}
$$

where A is a proportionality constant. 


\section{CHAPTER 2}

\section{The GlueX Experiment}

The GlueX experiment is part of the $12 \mathrm{GeV}$ upgrade project at the Thomas Jefferson National Accelerator Facility (TJNAF) in Newport News, Virginia. The primary physics goal of the experiment is to study the light meson spectrum and to search for exotic and hybrid mesons. The GlueX experiment is designed to collect high statistics and high quality data on the photoproduction of light mesons [13]. The quantum numbers of produced mesons will be determined via an amplitude analysis. A successful amplitude analysis requires high statistics and a hermetic detector that permits the determination of the fourmomenta of all decay products. The GlueX detector has been built in Hall D at Jefferson Lab and has been optimized for producing data specifically for amplitude analysis.

The GlueX experiment has been commissioned in late 2014 and through early 2015. The first physics quality beam was delivered in 2016. GlueX has collected 5 PB of physics data until the end of phase 1 in December of 2018. Currently we are collecting physics data in phase 2 of the GlueX experiment, which will continue for about five additional years.

In the GlueX experiment the Continuous Electron Beam Accelerator Facility (CEBAF) provides a $12 \mathrm{GeV}$ electron beam to the GlueX tagger hall. The electron beam is used to create tagged, linearly polarized photons via coherent bremsstrahlung in a diamond radiator. The photon beam subsequently interacts with a $30 \mathrm{~cm}$ long liquid hydrogen target at the center of the Gluex detector. In the next sections, the GlueX experimental setup will be described in summarized form from the start of the electron beam at CEBAF to the detectors used to measure the final state decay particles at Hall D.

\subsection{CEBAF Electron Beam}

Electrons are released from a gallium arsenide photo-cathode by a Laser beam that is pulsed with a frequency of either $249.5 \mathrm{MHz}$, or $499 \mathrm{MHz}$. The photo-electrons are then accelerated using two linear accelerators (linacs) in a race track configuration as shown in Fig. 2.1. Each accelerator contains super conducting Niobium radio frequency cavities. Niobium is 
a superconductor at very low temperature (2 Kelvin). The cavities are immersed in liquid helium and insulated from the outside temperature in so called cryomodules [28]. The electrons in the cavities ride the crest of electomagetic waves travelling the length of the cavities to acheive the desired energy with minimum heat loss. An electron bunch passes 5.5 times through the two linacs and the recirculating arcs before being delivered to the Hall D tagger with the desired energy. The CEBAF delivered the beam to Hall D at 249.5 $\mathrm{MHz}$ during the GlueX experiment. The accelerator precisely measures and controls the timing of the beam bunches. The timing information is made available to the experiment to determine the time at which the individual tagged photons interact with the target. For more details on the accelerator regarding beam production and delivery see ref. [29,30].

\section{CEBAF electron beam track and halls location}

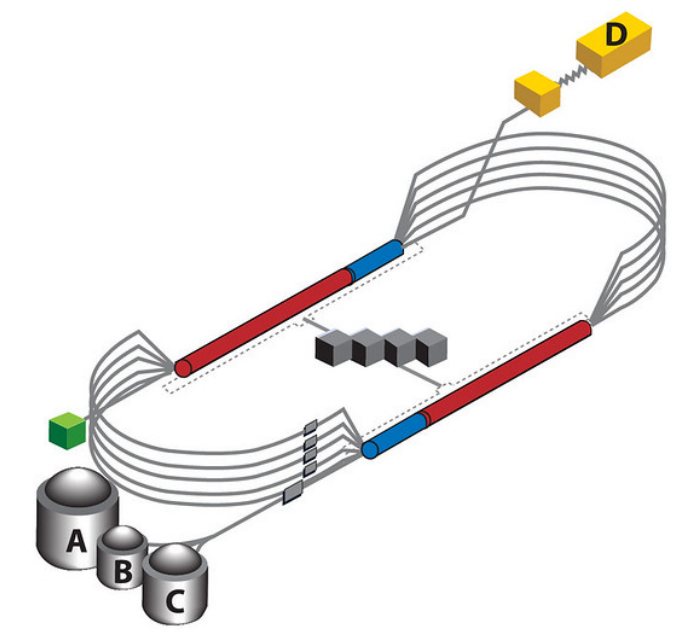

Figure 2.1: Schematic diagram for accelerator site, the injector in green, Hall D and tagger hall are in yellow. Halls A, B, and C in the opposite side.

\subsection{Coherent bremsstrahlung}

High energy, linearly polarized photons are produced by coherent Bremsstrahlung (CB) using a diamond radiator. Coherent Bremsstrahlung produces intensity enhancements (coherent peaks) in the normal bremsstrahlung spectrum because of the match between the incident electron momentum transfer and the reciprocal lattice vector calculated from the Fourier transform of the space crystal lattice [31]. Coherent bremsstrahlungs photons are 
linearly polarized and lead to overall linear polarization in the coherent peak regions. The CB is well descriped in the framework of Quantum Electro Dynamics (QED). Most of the emitted photons have very small angles relative to the incident electron beam according to the relation $\theta_{\gamma}=m / E$, where $\mathrm{m}$ and $\mathrm{E}$ are the mass and the initial energy of the electrons respectively [32]. The most energetic photons are emitted at $0^{\circ}$. More details about coherent bremsstrahlung, diamond fabrication, and characterization of the diamond radiator can be found in [32-34].

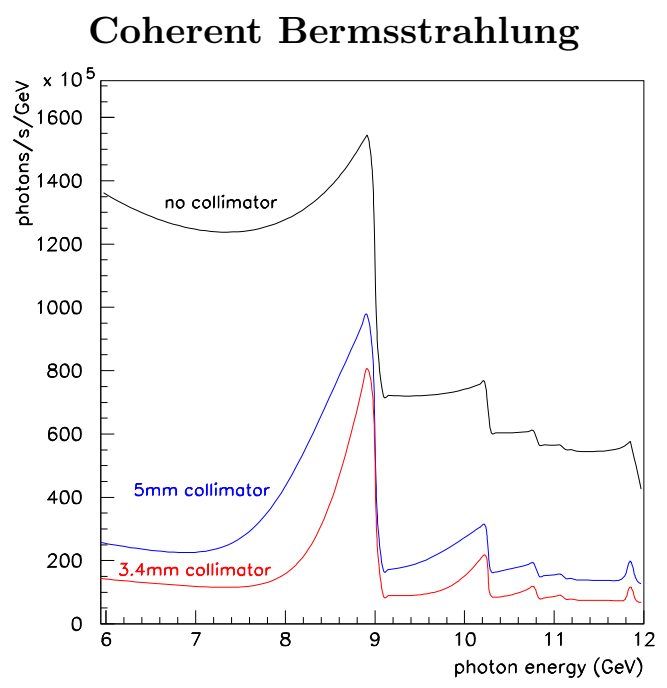

Figure 2.2: Uncollimated and collimated coherent bremsstrahlung spectrum, calculated for diamond radiator $15 \mu \mathrm{m}$ thick and a $1 \mu \mathrm{A}$ electron beam of $12 \mathrm{GeV}$ [35].

During the GlueX experiment an electron beam with an energy $\left(E_{\gamma}\right)$ of $6 \mathrm{GeV}<$ $E_{\gamma}<11.6 \mathrm{GeV}$, and intensities of $50 \mathrm{nA}<I<150 \mathrm{nA}$ was used. An optimum thickness for the GlueX diamond radiator was found to be $50 \mu \mathrm{m}$. Figure. 2.2 shows a calculated coherent bremsstrahlung spectrum for a $12 \mathrm{GeV}$ electron incident on a $15 \mu \mathrm{m}$ radiator, with a strong intensity enhancement near $9 \mathrm{GeV}$ as well as smaller enhancements at other energies [35]. The degree of polarization as well as the locations of the coherent photon peaks depend on the orientation of the crystal with respect to the incident electron beam. Therefore, the diamond crystal is mounted on a goniometer which can be adjusted to change the polarization direction of the linearly polarized photons. Four polarization angles have been used; each set of two angles are perpendicular to each other. The linearly polarized 


\section{Schematic Tagger Diagram}

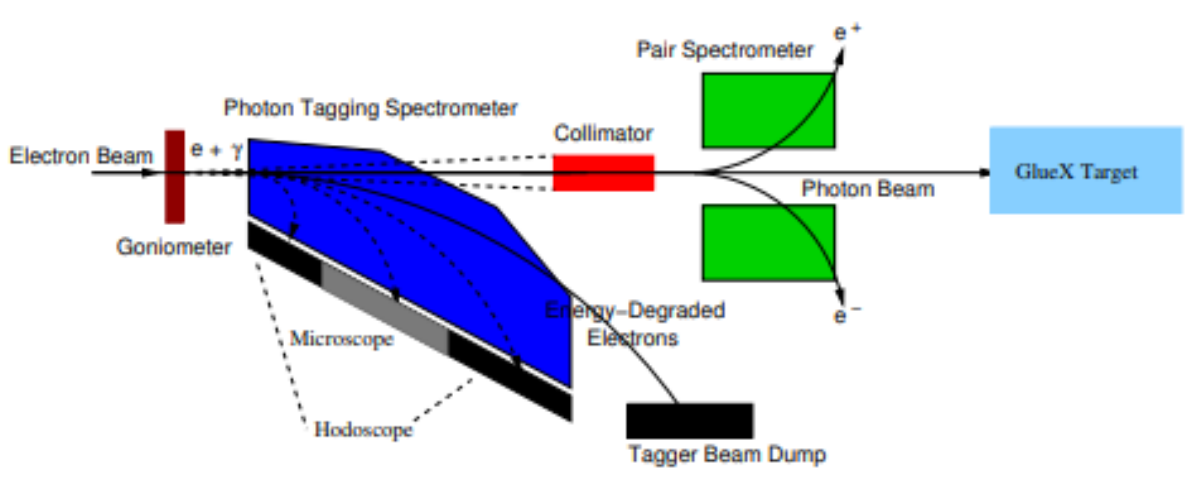

Figure 2.3: Tagger hall view, not to scale

photons are then tagged and transported through the GlueX beam line to interact with the target in the center of the GlueX detector. The tagging procedure, flux determination and polarization measurements are discussed in the next section.

\subsection{The GlueX Beam Line and Tagger Hall}

The linearly polarized photon beam travels $70 \mathrm{~m}$ until it reaches the Hall D beam collimator in the collimator cave. The collimation of the beam is essential in order to significantly enhance the photon polarization. The polarization for the coherent bremsstrahlung photons is maximal for a small photon angles along the beam line while incoherent photons have a broader angular distribution. Consequently the photon beam is collimated by a 5.0 mm collimator to select photons with angles smaller than $4 \mu \mathrm{rad}$ relative to the beam line. During part of the experiment, a $20 \mu \mathrm{m}$ thick diamond radiator has been used in combination with a $3.4 \mathrm{~mm}$ collimator. However this configuration was not sustainable for long period of times, specially at larger beam currents, because of radiation damage to the diamond.

Photons are tagged by measuring the energy of the electrons after they have interacted with the diamond. The electron beam passes through a dipole magnet located after the radiator. The magnetic field makes it possible to measure the final electron energy and thus determine the energy of the associated bremsstrahlung photon. A schematic diagram of the 
tagger hall is shown in Fig. 2.3 from [36]. Electrons that have not emitted a photon are swept out of the beam line by the dipole field and are redirected to an electron beam dump in the tagger hall. Electrons that emitted a bremsstrahlung photon are detected by the Tagger Hodoscope, TAGH (a highly segmented array of scintillators). From the location of the detector in the tagger spectrometer focal plane the electron energy and consequently the photon energy can be determined with a resolution ranging from 8.5 to $30 \mathrm{MeV}$. The TAGH scintellators can determine electron energies of $15 \%$ to $97 \%$ of the full electron beam energy range with a gap that corresponds to the energy range of the primary coherent peak. A high resolution detector, the Tagger Microscope (TAGM), is used to measure the energy range in the gap between TAGH and TAGM. In nominal GlueX operating conditions the TAGM covers a $1 \mathrm{GeV}$ energy range between $8.2 \mathrm{GeV}$ and $9.2 \mathrm{GeV}$. Given the initial electron energy from the accelerator $E_{o}$, and measured final electron energy (by the tagger) $E_{t a g}$, the tagged photon energy is given by $E_{\gamma}=E_{o}-E_{t a g}$.

The photon flux is measured using a Pair Spectrometer (PS). Its fundamental operating principle relies on the conversion of a photon to an electron positron pair. The produced electron and positron are swept out of the beam line by a dipole magnet and then detected by scintillator detectors. The PS is also used to determine the fraction of linearly polarized photons in the energy region of the coherent peak and for the energy calibration of the tagger detectors. The degree of polarization of the photon beam is measured by the Triplet Polarimeter (TPOL), where a polarized photon produces an electron positron pair when interacting with an atomic electron. The electron positron pair as well as the recoiling electron are detected. The PS detects $e^{+} e^{-}$pair in coincidence with hits in the recoil detector in Hall D. The recoiling electron is detected by the TPOL silicon strip detector (SSD). The photon beam polarization is determined from the azimuthal angular distribution of the recoil electron.

The determination of the photon beam intensity is crucial for the determination of absolute cross sections. For photon beam intensity determination purpose, a known fraction of the photon beam is converted to $e^{+} e^{-}$pairs and counted in the PS as a function of energy. The energy of the $e^{+} e^{-}$pairs can be reconstructed by measuring their momenta in the PS 


\section{Flux and polarization determination}

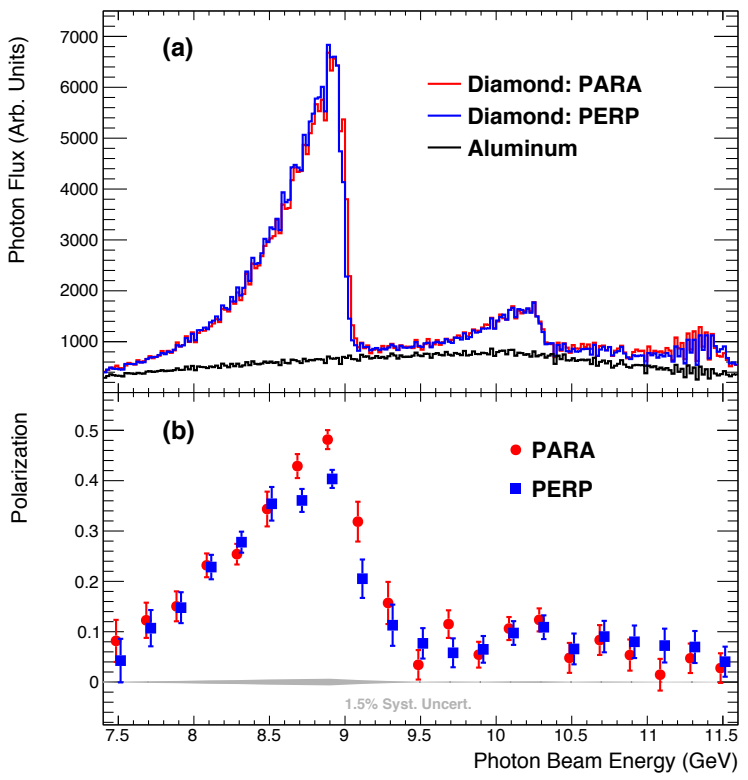

Figure 2.4: Photon flux versus energy as determined by PS (a). TPOL polarization determination as a function of energy (b). The polarization planes that are parallel and perpendicullar to the horizontal are denoted by PARA and PERP [37].

arms after they were deflected by the $1.8 \mathrm{~T}$ magnetic field [38]. The integrated number of photons over the run period is obtained as a function of beam energy for each counter in TAGM and TAGH. The PS calibration parameter used in the flux measurements is determined using special calibration runs, where the Total Absorption Counter (TAC) is used. It is a small calorimeter that can be inserted directly in the photon beam to count the number of beam photons as a function of energy. The special TAC runs used for absolute flux determination are performed at much reduced beam intensity to reduce the accidental tagging coincident rates. Data from both TAC and PS are used for the absolute flux calibration by counting the number of pairs in the PS with the same energy as seen by the TAC. The expected uncertainty in flux determination is $2 \%-5 \%$. The exact value of the systematic error associated with flux determination is currently being evaluated by the collaboration. An example of flux and polarization determination as a function of energy is shown in Fig. 2.4. The measured photon flux spectrum by the PS with the coherent peaks enhancements is shown in Fig. 2.4(a) as a function of the photon energy for two orientations of the diamond radiator, and an amorphous one where CB does not occur. The measured 
polarization by TPOL for the two diamond orientations is shown in Fig. 2.4(b), with an average polarization of $45 \%$ in the coherent peak.

For the low energy data set, the Pair Spectrometer magnet was adjusted to measure the photon flux in the energy range $2.9 \mathrm{GeV} \leq E_{\gamma} \leq 5.9 \mathrm{GeV}$ while for standard GlueX measurements the Pair Spectrometer determines the photon flux for photon energies between 6.2 and $11.6 \mathrm{GeV}$.

\subsection{The Gluex Detector Components}

The GlueX detector is a hermetic solenoid-based detector. It detects the final state neutral and charged particles in a nearly $4 \pi$ solid angle. A schematic diagram for the GlueX detector, and the beam line is shown in Fig. 2.5. A 2-Tesla solenoid magnet is the outer most layer of the detector surrounding the proton target. The magnetic field is needed for charge and momentum determination.

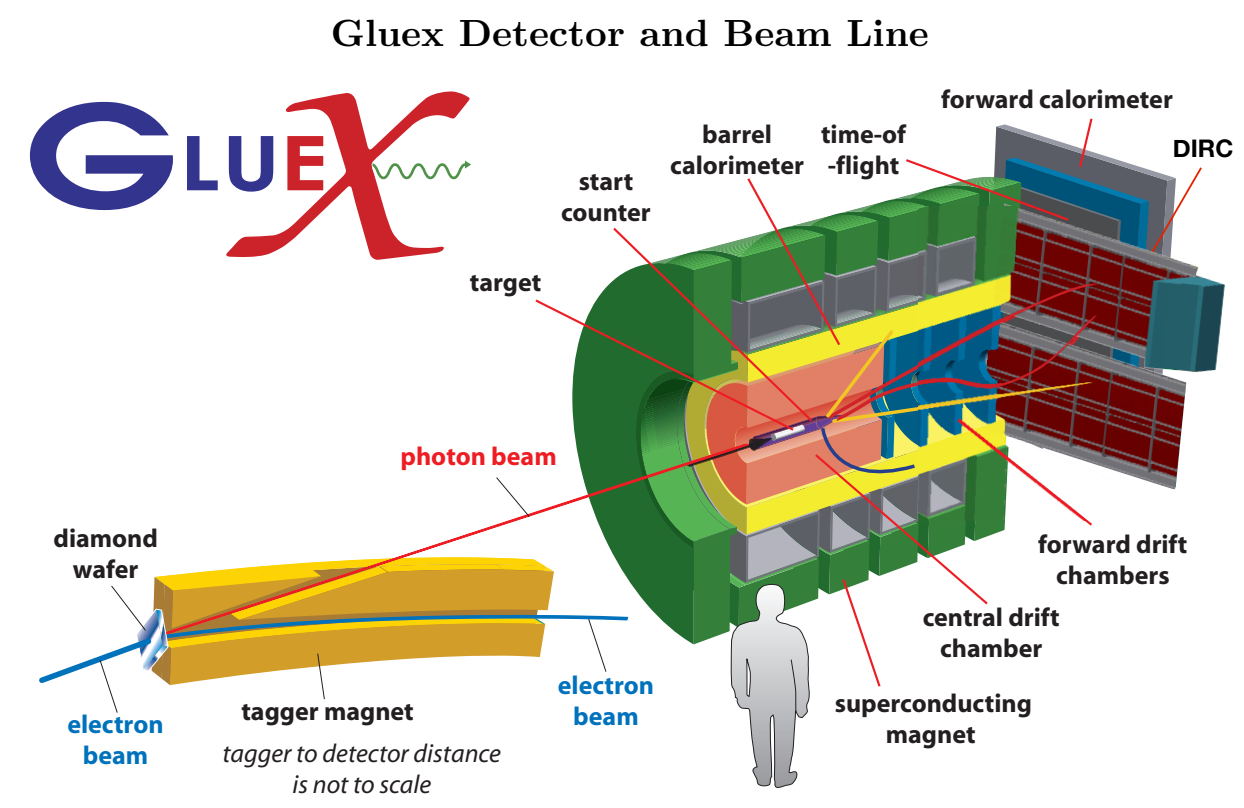

Figure 2.5: Schematic diagram for the Gluex detector and beam line. The distance between the diamond wafer and the liquid hydrogen target is $70 \mathrm{~m}$ in reality.

The Start Counter (SC) is the closest detector to the target. The main purpose of the SC is to identify the electron beam bucket associated with the detected particles. The Central Drift Chamber (CDC) is surrounding the SC. It is used for charged particle tracking 
and particle identification. It covers a wide range of angles from $11^{\circ}$ to $126^{\circ}$ with respect to the photon beam. The tracks of forward going charged particle with polar angles below $11^{\circ}$ are determined using the Forward Drift Chambers (FDC). The Barrel Calorimeter (BCAL) encloses the CDC and is used to determine the energies of decay photons. The energy of forward going photons is measured using the Forward Calorimeter (FCAL). The Time Of Flight (TOF) detector in front of the FCAL provides timing information for forward going charged particles. It is complemented by time-of-flight measurements by the BCAL and $\frac{d E}{d x}$ measurements from the CDC. The combined time-of-flight and $\frac{d E}{d x}$ information is used for particle identification (PID).

\subsection{Start Counter (SC)}

The SC surrounds the target and consists of an array of 30 scintillators arranged around the target as shown in Fig. 2.6. Each scintillator has three distinguished geometrical sections, straight, bend, and nose as shown in figure 2.7. Tedlar wrapping, attached to a plastic collar at the upstream end of the SC assembly is used to make it light-tight. Figure 2.6 shows the SC ready to be inserted into the GlueX detector. It was designed to operate at tagged photon beam intensities of up to $10^{8} \mathrm{\gamma} / \mathrm{s}$ in the primary coherent peak where the photon energy ranges from 8.2 to $9.2 \mathrm{GeV}$. During the GlueX phase I operation, the photon intensities were up to $0.5 \times 10^{7} \gamma / s$, while in Phase II operation, the photon intensities increased to $\sim 1 \times 10^{7} \gamma / \mathrm{s}$. At these photon intensities each scintillator measured a particle rate of about $200 \mathrm{KHz}$.

The overall time resolution for the start counter is less than 300 ps which is well below the 350 ps design specification. The time resolution is sufficient to identify individual electron beam bunches and the coincident photon from which the event is created. The SC timing signal is independent of the particle type and trajectory because of its location very close to the target. The overall start counter efficiency is $97.2 \pm 0.1 \%$. The efficiency can vary depending on which start counter geometrical section was hit ( straight, bend, or nose geometry). The SC light sensors are magnetic field insensitive Silicon PhotoMultiplier detectors $(\mathrm{SiPM})$. The electronics provides two signals per scintillator paddle, one for the 


\section{SC mounted the GlueX target}

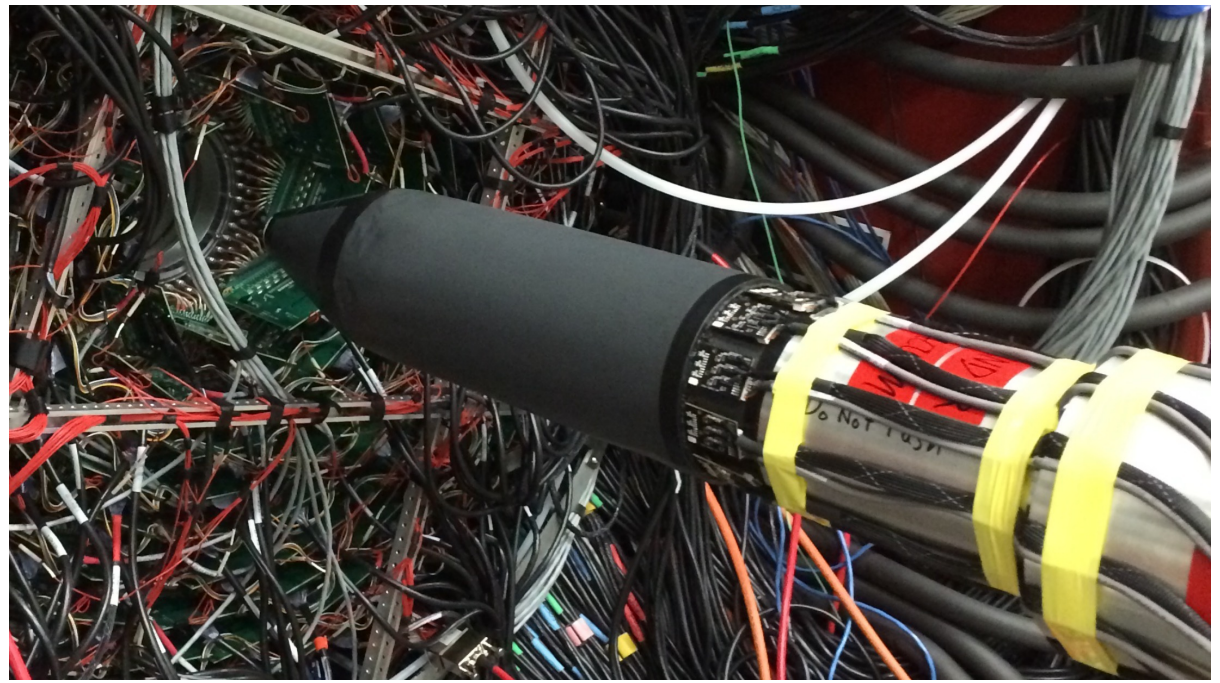

Figure 2.6: SC mounted the GlueX target. The beam travels down the central axis of the SC from right to left. During run operations the SC inserted in the bore of the central drift chamber shown at the top left corner.

Flash Analogue to Digital Converter (FADC), and the other is sent to a discriminator and then to a Time to Digital Converter (TDC). The SC timing signal must be calibrated to determine time-walk, light propagation time, and light attenuation corrections. For more details about design, simulation, fabrication, calibration, and performance of the SC see reference [39].

\subsubsection{SC Calibration}

The first calibration step is to correct the TDC time for time shifts that result from variations in the pulse amplitude (time walk). Time walk occurs because of the time difference when small and large amplitudes pulses cross a fixed threshold. The time information provided by the FADC is independent of the pulse amplitude and therefore can be used as a reference. The methods used to correct for the time walk and light attenuation are described in details at reference [39]. The time interval between a particle crossing the scintillator and the scintillation light detection at the $\mathrm{SiPM}$ is called the propagation time (PT), see 


\section{SC paddle geometry}

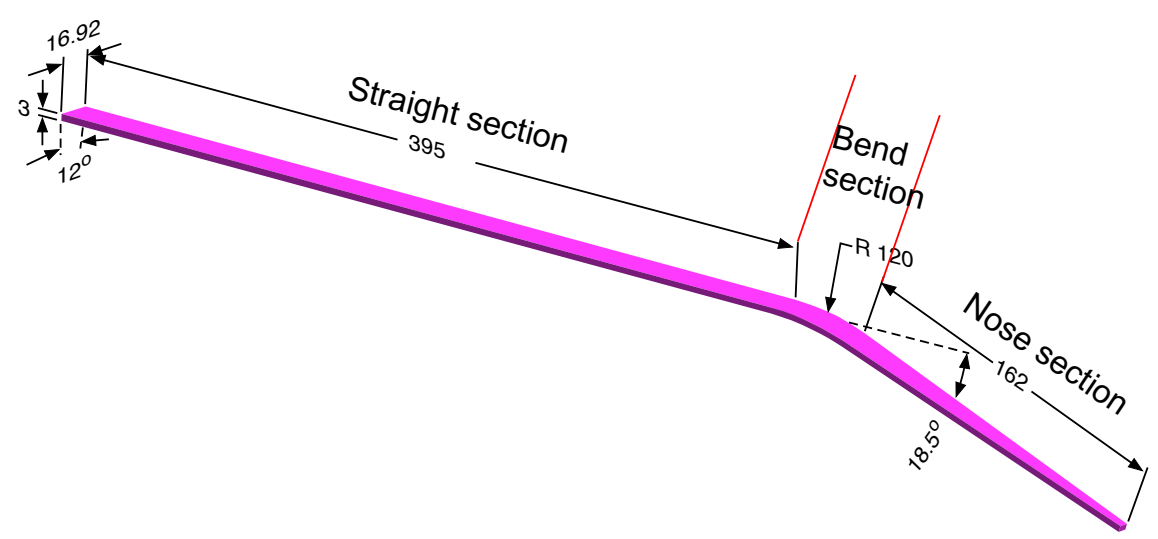

Figure 2.7: SC paddle geometry. Straight, bend, and nose sections

Fig. 2.8. It is defined by equation 2.1 .

$$
P T=T_{s c}^{w c}-T_{s c}^{f t}-T_{r f}
$$

Where:

- $T_{s c}^{w c}$ is the SC time corrected for time walk.

- $T_{s c}^{f t}$ is the flight time from the vertex to the SC, corrected to the target center.

- $T_{r f}$ is the RF time given at the center of the target. It defines the start time of a physics trigger event.

The SC propagation time correction has been updated since the SC article [39] has been published. It now uses a fit of the SC time versus the $\mathrm{z}$ coordinate of the intersection of the charged particle with the SC. Since the purpose of the SC is to determine the start time of an event, the calibration procedure must not depend on the SC time itself. Therefore, the RF time $\left(T_{r f}\right)$ in eqation 2.1, is determined by a good track based on timing and PID information from the TOF detector and does not involve the SC. A track confidence level (CL) is calculated for the model applied to the fitted data from the drift tracking chambers (CDC/FDC). It is defined as the integral of the $\chi^{2}$ probability distributions from $\left[\chi^{2}, \infty\right]$, and represents the goodness of the fit of the tracks. For detailed information about track reconstruction steps from the tracking detectors, see [37]. The selection of a good 


\section{SC Propagation Time}

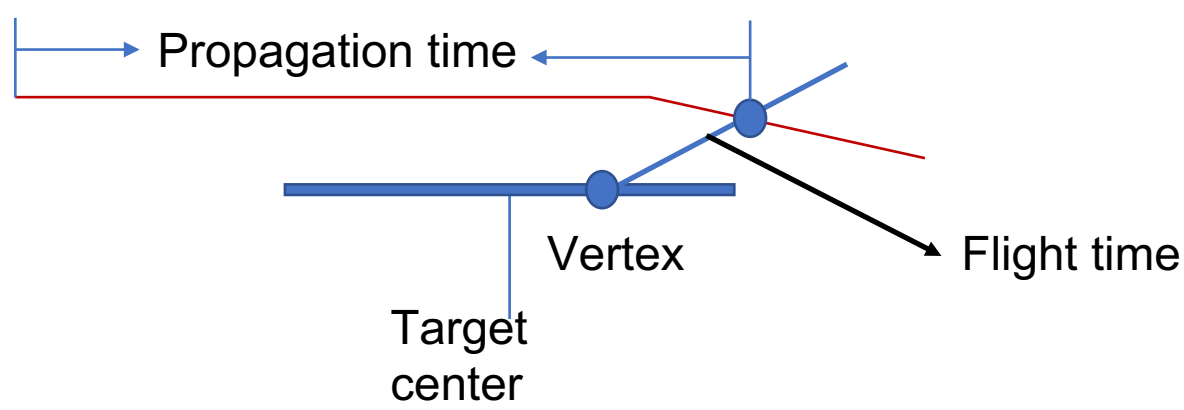

Figure 2.8: Sketch to illustrate the SC propagation time

SC Propagation Time

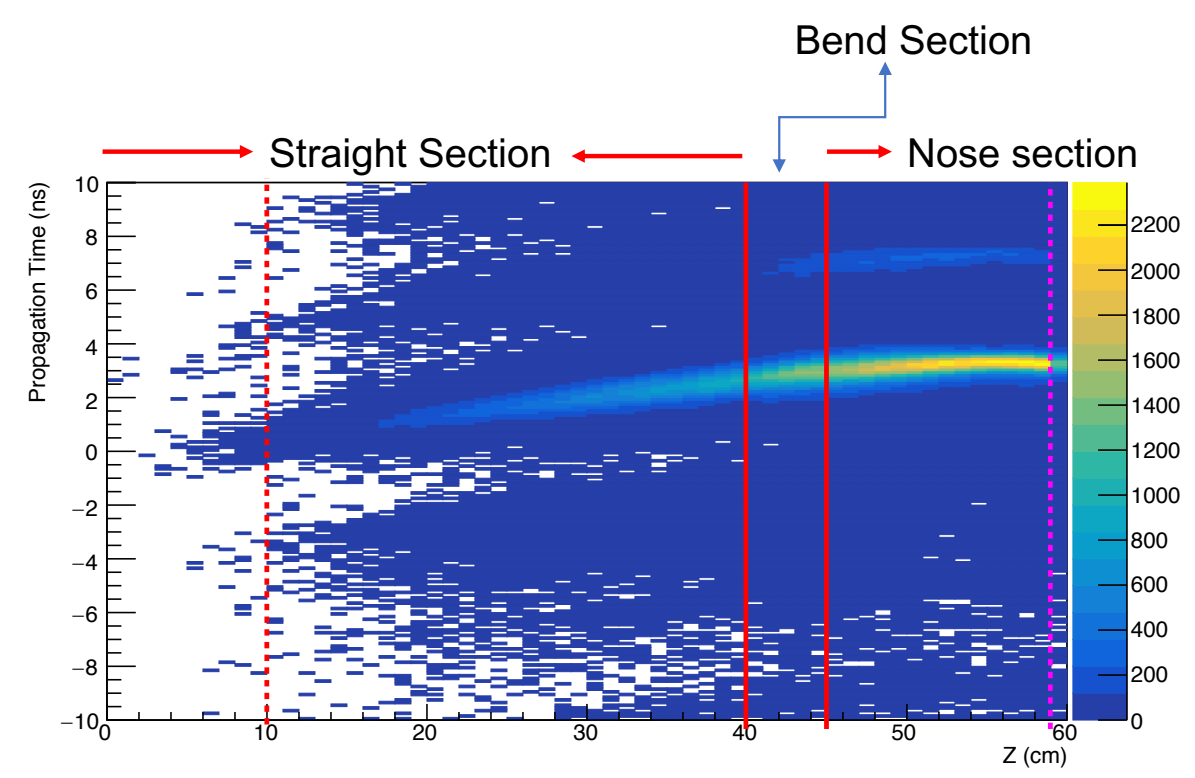

Figure 2.9: Propagation time $(\mathrm{ns}) \mathrm{vs} \mathrm{z}(\mathrm{cm})$. The red dotted line denotes the first $10 \mathrm{~cm}$ of the straight section. The length from the red dotted line to the first solid line denotes the rest of the straight section. The bend section is located between the red solid lines. The nose section starts right after the second solid red line. The magenta line is at the beginning of the last nose section interval. 


\section{Gaussian fits for SC propagation time peaks}
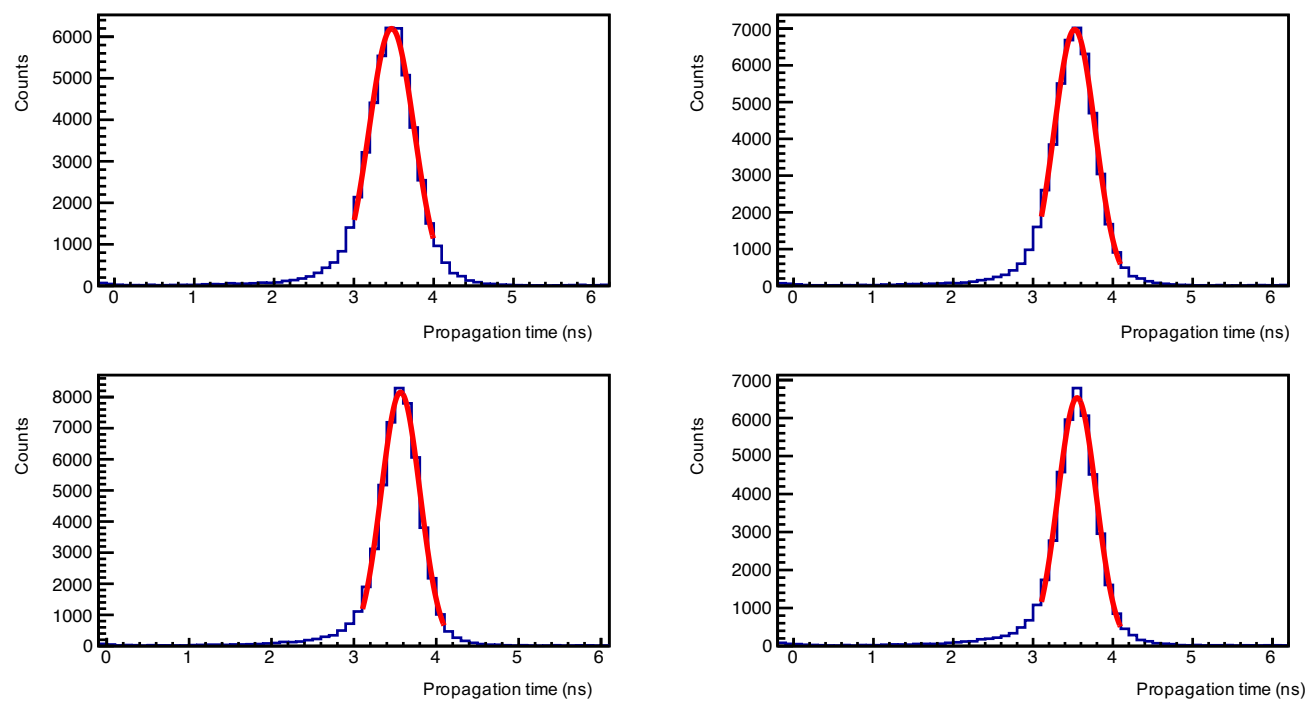

Figure 2.10: Gaussian fits for SC propagation time peaks for four selected intervals in the nose section.

reconstructed track, from tracking detectors, for the SC PT calibration is determined by the following criteria:

- Track confidence level (CL) > 0.0027 .

- Track originated within the target geometry with a radial cut $(r<0.5 \mathrm{~cm})$, where $r=\sqrt{x^{2}+y^{2}}$, and $(\mathrm{x}, \mathrm{y}, \mathrm{z})$ are the coordinates of the track vertex.

- In addition, a z-coordinate cut from $50 \mathrm{~cm}$ to $80 \mathrm{~cm}$ is applied, making sure the vertex is located inside the hydrogen target cell.

A plot of a typical SC propagation time as a function of the hit location $\mathrm{z}$, measured along the SC paddle to the SiPM is shown in Fig. 2.9. The main coincidence peak as well as other out of time peaks are clearly shown as band structures. The first $10 \mathrm{~cm}$ upstream of the straight section can be ignored because of a lack of statistics compared to the other parts of the SC. The remaining, approximately $30 \mathrm{~cm}$, of the straight section was divided into 6 intervals, while the bend section was divided into 3 intervals and the nose section was segmented into 9 intervals. The propagation time distribution is then obtained for each of the eighteen SC intervals as well as the central $\mathrm{z}$ value for all the thirty sectors of the SC. The propagation time peak for each of the intervals is fitted with a Gaussian as in Fig. 


\section{SC Propagation Time Fits}
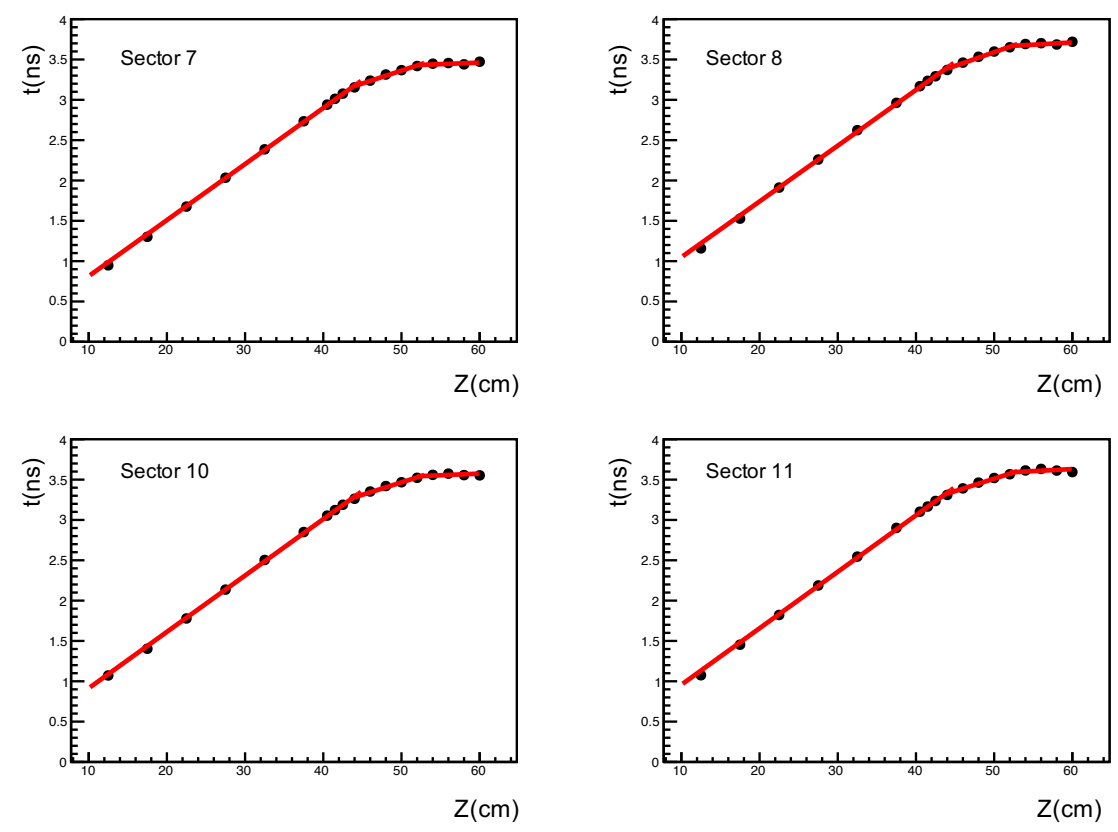

Figure 2.11: Propagation time (ns) vs z $(\mathrm{cm})$. Three linear fitted regions

2.10, and the mean propagation time for each interval is determined. Three linear fits of the propagation time as a function of $\mathrm{z}$ were performed for each paddle, since there are three different effective light propagation speeds associated with the geometrical shape of the SC $\left(f_{i}(z)=a_{i}+b_{i} z\right)$. These fit parameters are saved in the Calibration Constants Data Base (CCDB). The inverse of $b_{i}$ is the effective light propagation speed in the scintillator material. It ranges from $13 \mathrm{~cm} / \mathrm{ns}$ to $25 \mathrm{~cm} / \mathrm{ns}$, in most of the paddles. Figure 2.11 shows the fits for four different paddles of the SC. For each hit location(z) the propagation time correction is applied and the corrected time is calculated as in equation 2.2. The boundaries between the geometrical region were made continuous by allowing the fits to extend for a distance $(\delta z)$ into each side and the intersection point between the two lines was used as an adjusted boundary.

$$
P T_{\text {Corrected }}=P T-f_{i}(z)
$$

The corrected propagation time as a function of the hit location $\mathrm{z}$ is shown in figure 2.12. The propagation time after correction has a flat distribution near zero as expected. 
SC Propagation Time After Correction

Corrected Time vs. Z

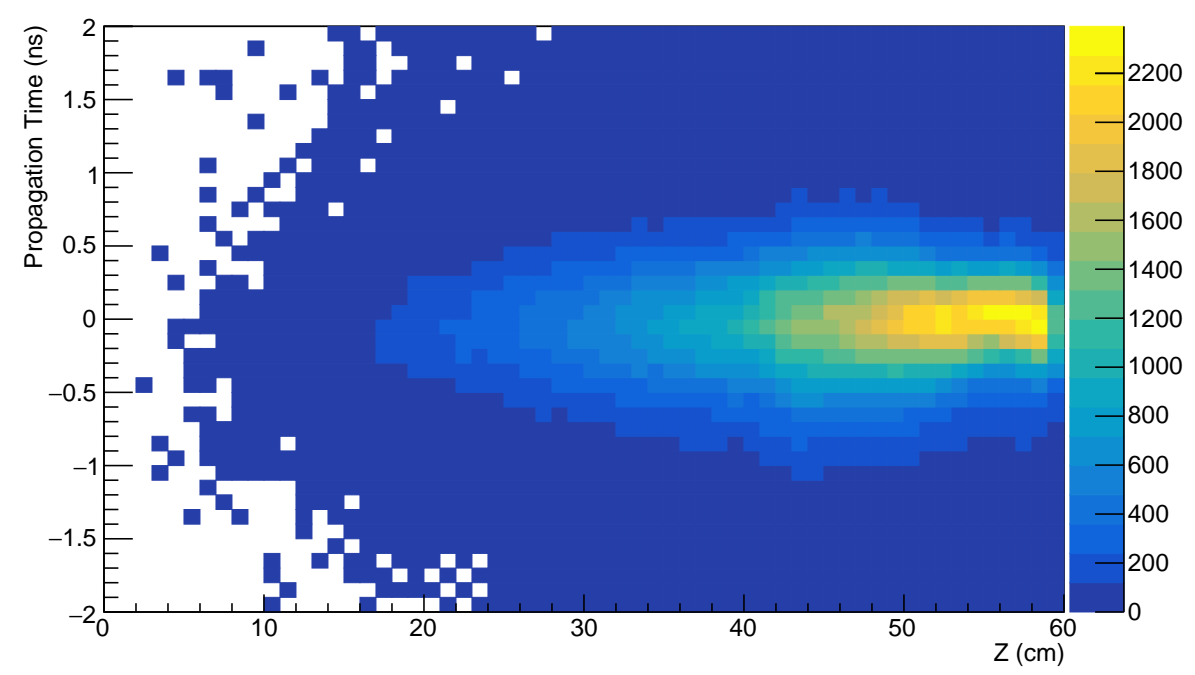

Figure 2.12: SC propagation time (ns) vs $\mathrm{z}(\mathrm{cm})$. The main $\mathrm{RF}$ bunch band is aligned at about 0 ns after correction.

\subsubsection{SC Efficiency}

The efficiency of the start counter is defined as the ratio of the number of recorded SC hits to the number of tracks reconstructed from the (CDC/FDC) through the start counter, i.e., the tracking algorithm indicates a particle that has passed through the SC (projected tracks to the SC). To calculate efficiency, one needs to use these projected tracks where no SC timing information has been used at their reconstruction. The track reconstruction procedure is discussed in details in ref. [40]. The following conditions were used to select good quality tracks:

1. The track must be reconstructed with at least 14 hits through out the Gluex detector components.

2. The track must have a CL greater than $2.7 \times 10^{-3}$.

3. It must originate from within the target geometry.

4. The charged particle track is matched either to the BCAL or (FCAL, and TOF) detectors. 


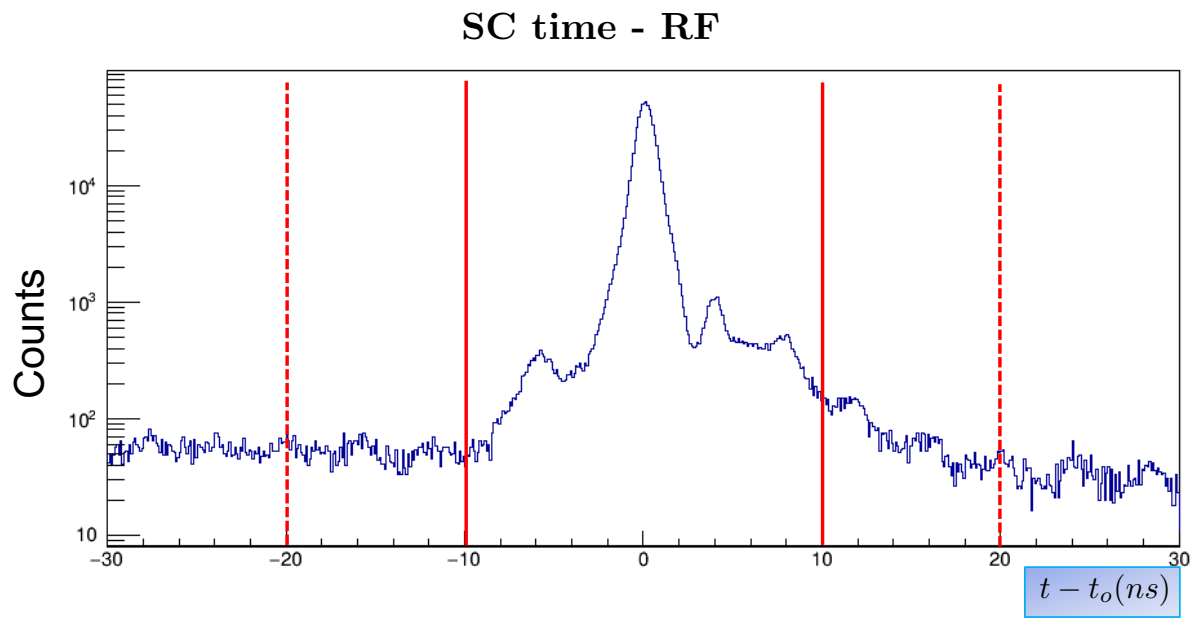

Figure 2.13: The total number of the SC recorded hit is determined within the two solid lines. The dashed line in both sides is the end of each side band intervals used for accidental calculations.

The total number of projected tracks that satisfy these conditions is defined as $N_{p}$, and their corresponding RF times as $t_{o}$. The recorded $\mathrm{SC}$ hits are determined as well as the actual SC corrected hit time $t$. The spectrum of the time difference between the RF time and the SC time matched for hits is shown in figure 2.13 .

Once the sector number of a projected SC hit is identified, one checks if the same sector or its neighboring paddle had a hit, and the total number of the recorded hits $N_{r}$ in the coincidence time peak is identified. The total number of the accidental hits $\left(N_{a}\right)$ can be calculated using the side band subtraction method on a $10 \mathrm{~ns}$ interval on either side of the main interval. The efficiency $\epsilon_{i}$ for each scintillator is then given by

$$
\epsilon_{i}=\frac{N_{r}-N_{a}}{N_{p}}
$$

The SC efficiency calculated during the Spring 2018 run period for only a single run is shown in Fig. 2.14. The overall SC efficiency $\epsilon$ is calculated using equation 2.4. Figure 2.14 shows the overall SC efficiency $\epsilon=97.2 \pm 0.1 \%$ in (a), and the SC efficiency for each geometrical sections in (b).

$$
\epsilon=\frac{1}{30} \sum_{i}^{30} \epsilon_{i}
$$




\section{SC Efficiency}
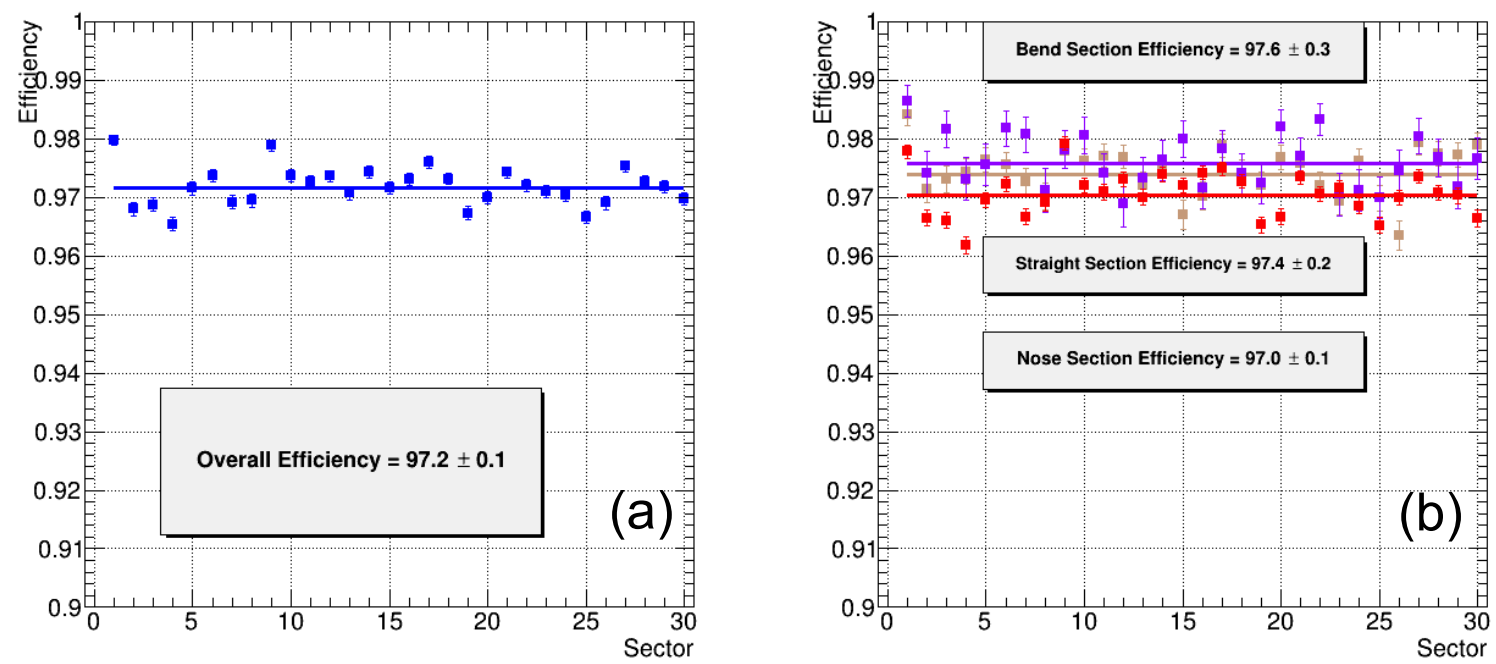

Figure 2.14: The overall SC efficiency (a). The Straight (brown), bend (violet), and nose (red) section efficiencies (b).

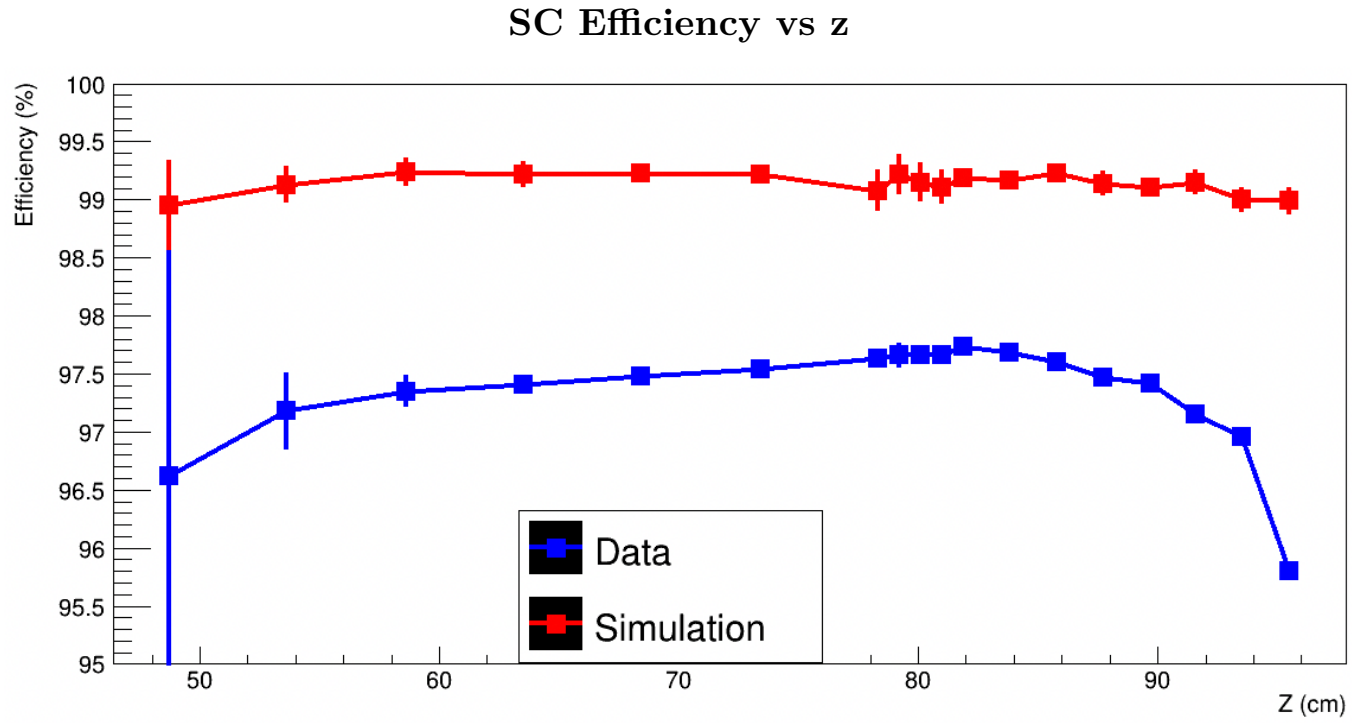

Figure 2.15: The SC efficiency as a function $\mathrm{z}$ coordinate of the charged particle track intersection with the SC paddle. 


\section{Efficiency Distributions.}
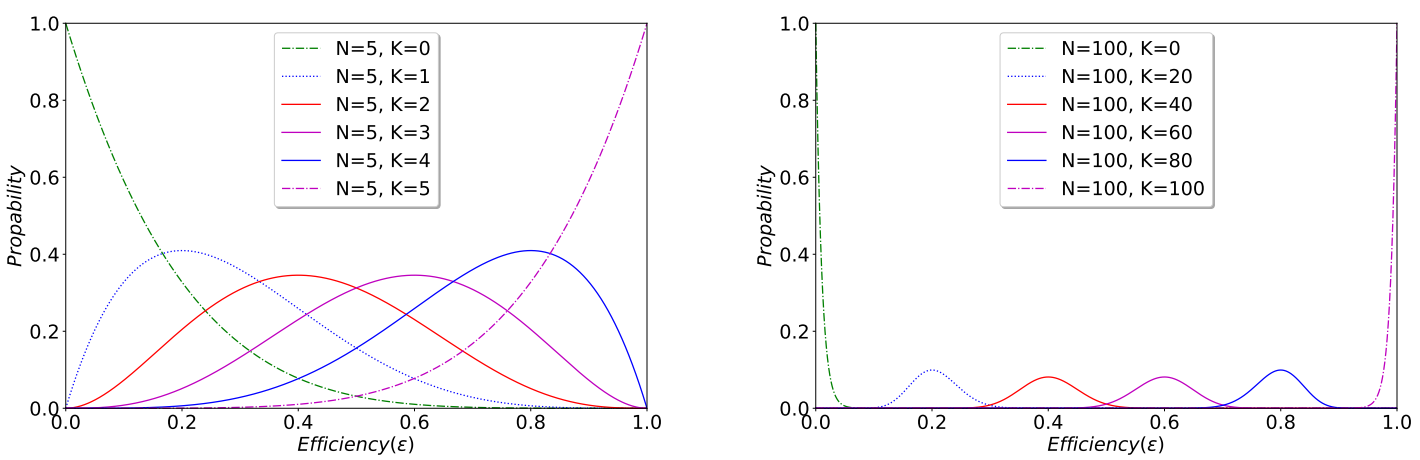

Figure 2.16: Probability distribution for efficiency calculation for total number of events $\mathrm{N}=5$, and $\mathrm{N}=100$.

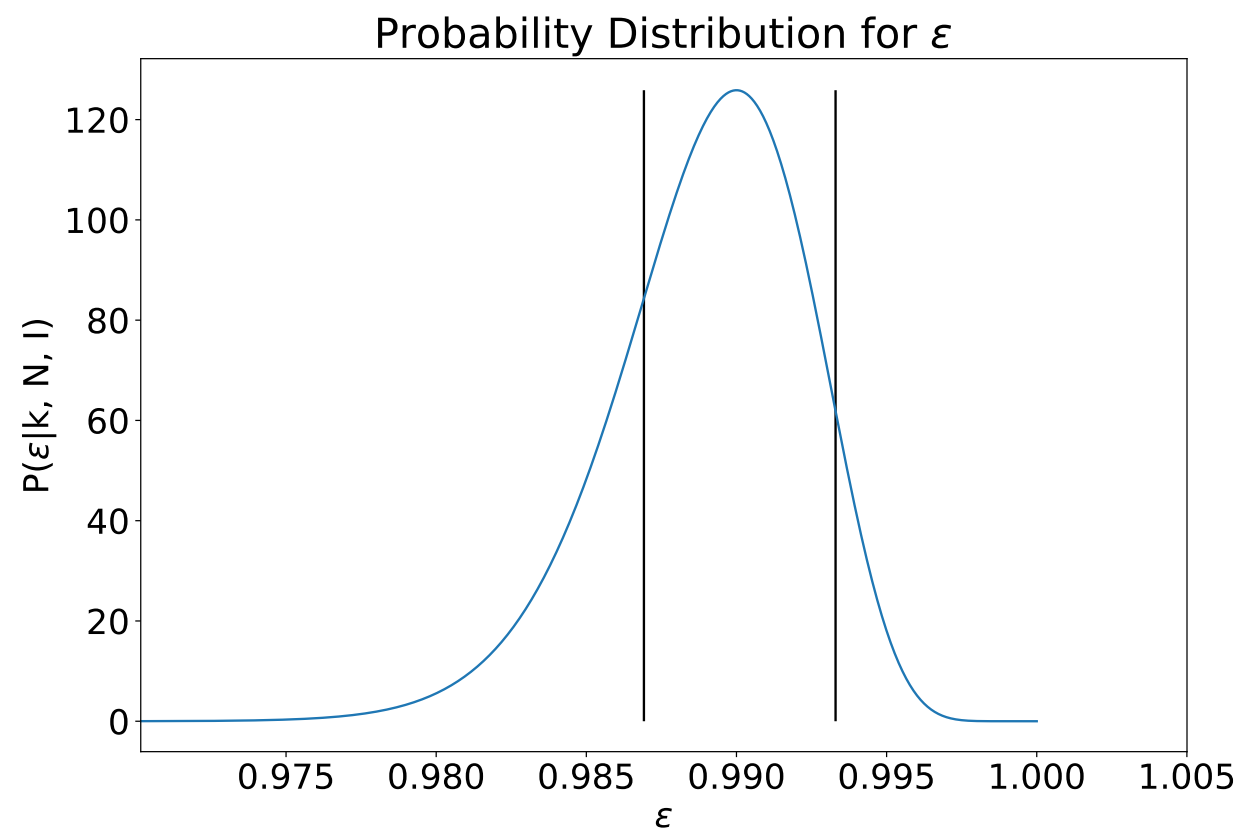

Figure 2.17: Efficiency error estimation using the Baysian method for $99 \%$ efficiency of $\mathrm{N}=1000$. 
The efficiency in the nose section is slightly smaller than the straight and bend sections efficiencies. This is a consequence of very difficult tracking at the far tip of the nose section where the width of the SC paddle becomes less than $2 \mathrm{~mm}$. The comparison between the SC efficiency from data and simulation is presented in Fig. 2.15. The simulated Monte Carlo (MC) sample is generated using one of the first generators to simulate the photoproduction cross section, named bggen, and was based on Pythia [41]. In general, the simulated efficiencies are $\sim 1.5 \%$ higher than the experimental ones. In the most forward $2-4 \mathrm{~cm}$ the simulation overestimates the experimental ones by $2-4 \%$. The simulated efficiency is almost flat as a function of $\mathrm{z}$ with an average value of $99.2 \%$. The SC data efficiency drops by at least $1 \%$ from the adjacent points at the tip of the nose most likely due to the reasons of difficult tracking in tip of the nose region.

The determination of the efficiency uncertainity is discussed in details in ref. [42]. There are two commonly used approaches to estimate the efficiency uncertainities using "Poisson", and "binomial" errors. Both methods produce absurd results in limiting cases as discussed in ref. [42]. According to a Bayesian analysis the probability $P(\epsilon \mid K, N, I)$ of having an efficiency $\epsilon$ from a total number of $\mathrm{N}$ events, where $\mathrm{K}$ events passed the cut conditions (I) is given by

$$
P(\epsilon \mid K, N, I)=\frac{\Gamma(N+2)}{\Gamma(K+1) \Gamma(N-K)} \epsilon^{K}(1-\epsilon)^{N-K}
$$

Figure 2.16 shows the probability $(P(\epsilon \mid K, N, I))$ as a function of $\epsilon$ for fixed values of the total number of events $\mathrm{N}=5$, and $\mathrm{N}=100$ using various number of event $\mathrm{K}$, that passed condition I. In all cases the probability is zero to have an efficiency smaller than zero or greater than one. There are two necessary conditions, the probability is zero for $\epsilon=0$ except for $\mathrm{K}=0$. Similarly, the probability is zero for $\epsilon=1$ except when $\mathrm{K}=\mathrm{N}$. For any other values, the probability peaks at the most probable value of the efficiency. As the sample number $(\mathrm{N})$ increases the probability peaks at the exact efficiency value with a narrow distribution around the most probable value. 

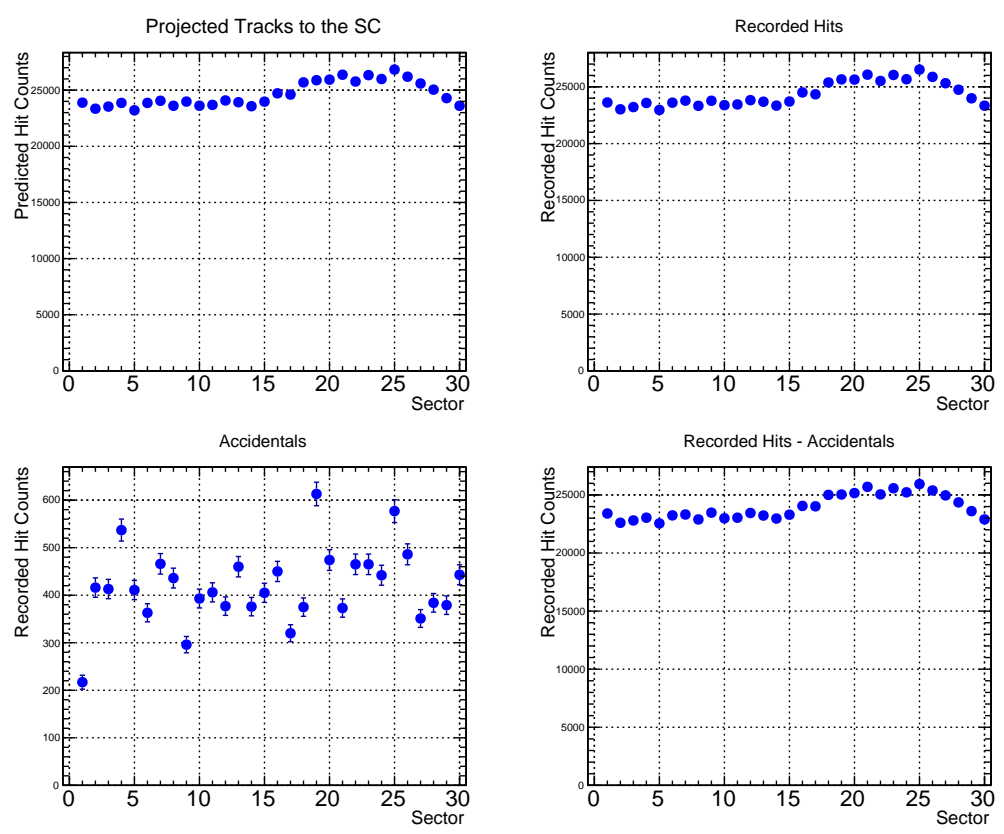

Figure 2.18: Predicted $\left(N_{p}\right)$ and recorded $\operatorname{tracks}\left(N_{r}\right)$ to the SC. The accidental tracks $\left(N_{a}\right)$ and the difference $N_{r}-N_{a}$ are shown in the bottom plots.

Since the total number of hits at each SC paddle is mathematically going to infinity, the probability and consequently the error becomes very small around an exact efficiency value. The error calculation in the efficiency given by eqn.(2.4) is determined by error propagation when dividing the two histograms of the numerator and denominator. Since N is very large, and $\mathrm{K}$ is not zero nor equals $\mathrm{N}$, the error in the efficiency using simple error propagation method is an overestimate. Consider an example of 990 events passed the condition I from a total number of 1000 event. The efficiency and its error calculated using the error propagation method is $0.99 \pm 0.04$, while the error from the probability distribution within $68 \%$ cumulative probability is less than 0.003 , see Fig. 2.17. A typical projected and recorded SC tracks count are shown in Fig. 2.18.

\subsubsection{SC Time Resolution}

The SC time resolution is discussed in the performance section of ref. [39]. It is calculated for all the 30 scintillators and for each of the geometrical sections. The time resolution is mostly uniform among all the SC paddles with values well below the 350 ns design specification. 


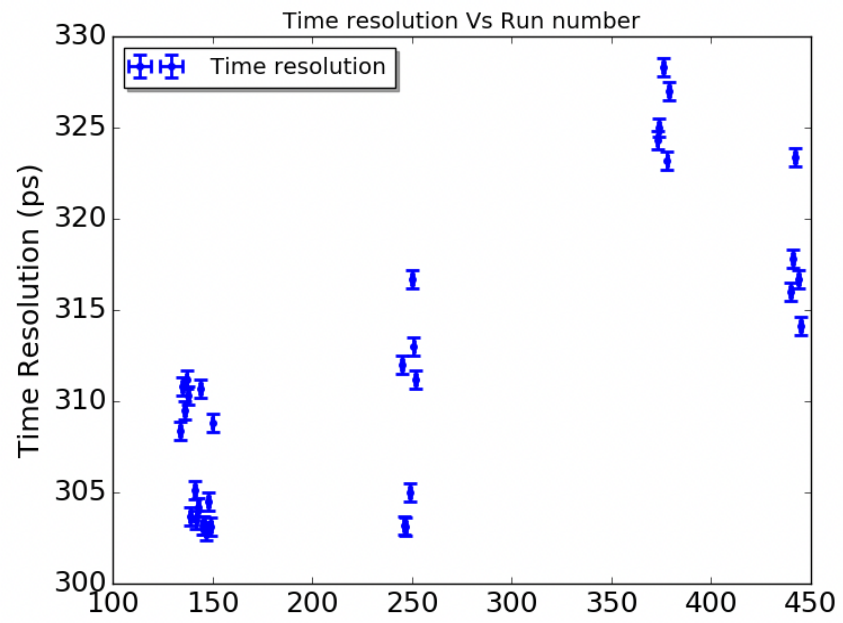

Figure 2.19: The SC Time resolution during Spring 2018 run period as a function of the run number. These runs started with $41 \mathrm{NNN}$, where $\mathrm{NNN}$ is the $\mathrm{x}$-axis number.

These values are enough to identify the individual electron beam bunches delivered by the CEBAF within $94 \%$ accuracy for $500 \mathrm{MHz}$ operation [39]. Figure 2.19 shows a time series of the average start counter time resolution during the Spring 2018 run period. The period started at run number 41NNN. The run numbers shown in Fig. 2.19 started at 41100 and ended at 41450 . The two digits 41 were omitted from the plot. The average SC time resolution was 307 ps after several years of operation with no signs of deterioration.

\subsection{Central Drift Chamber}

The CDC surrounds the SC, and it is one of the charged particles tracking detectors in the Gluex experiment. It is a straw tube drift chamber covering polar angles between $6^{\circ}$ and $168^{\circ}$. The CDC consists of 28 layers of closely-packed straw tubes. Twelve of these layers have an axial arrangement while the rest of the layers have stereo angles of $\pm 6^{\circ}$ with respect to the axial straws, see Fig. 2.20, in order to provide z-position information along the beam line. Each of these tubes are $0.775 \mathrm{~cm}$ in radius and $150 \mathrm{~cm}$ long. A $20 \mu \mathrm{m}$ diameter wire runs down the center of each tube and is held at high voltage (typically 2100 V during normal operation) with respect to the tube surface. The volume of the tube is filled with a gas mixture of $50 \%$ argon and $50 \%$ carbon dioxide at atmospheric pressure. 


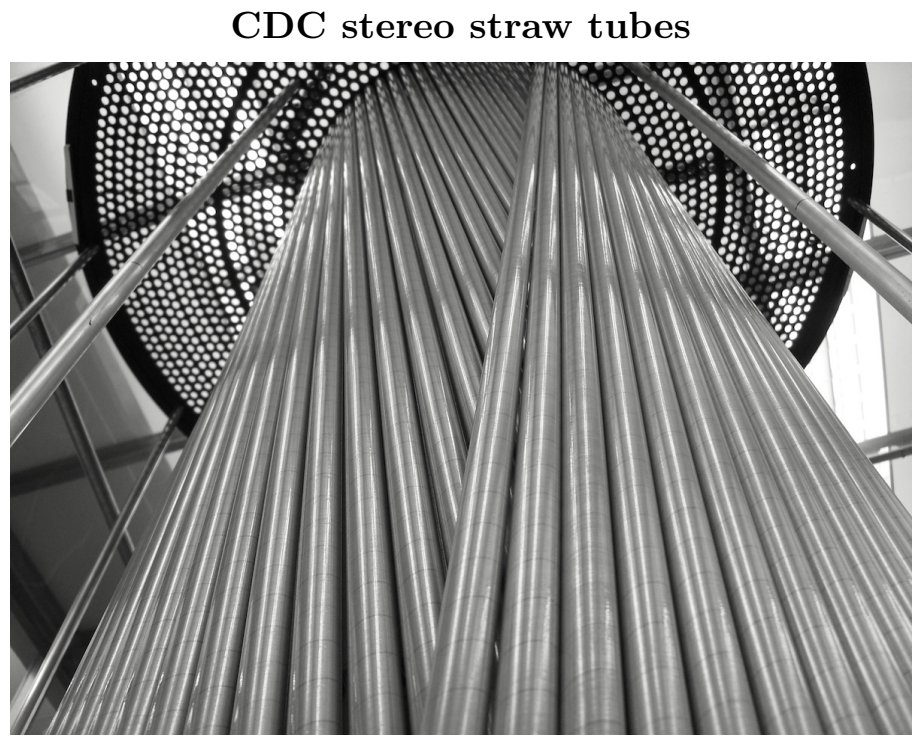

Figure 2.20: CDC stereo straw tubes. Partially completed layer of stereo straw tubes, during CDC construction, surrounding a layer of straw tubes at the opposite angle.

A passage of a charged particle through the straw will ionize the gas and the free electrons drift toward the wire. The initial ionization charge is multiplied by the high electric field near the wires and electron avalanche is measured by the readout electronics. The ionization charge is proportional to the energy lost by the particle. CDC pulse information from the anode wires are read out using 72-channel $125 \mathrm{MHz}$ flash ADCs. More details about the electronics can be found at references [37], [43], and [44].

The CDC also measures the energy deposition along the length of the track in the straws. The amount of energy deposited per unit length depends on the momentum and the particle type. At low momentum, this information can be used to distinguish between protons and mesons up to roughly $1 \mathrm{GeV}$. For more details about calibration and performance of the CDC see reference [45].

\subsection{Forward Drift Chamber}

The FDC is a cathode strip chamber covering polar angles between $1^{\circ}$ and $20^{\circ}$, and is located down stream of the CDC inside the magnetic field. The forward region of the detector has a very large particle density and therefore multi-track separation capability is 


\section{FDC package outlook}

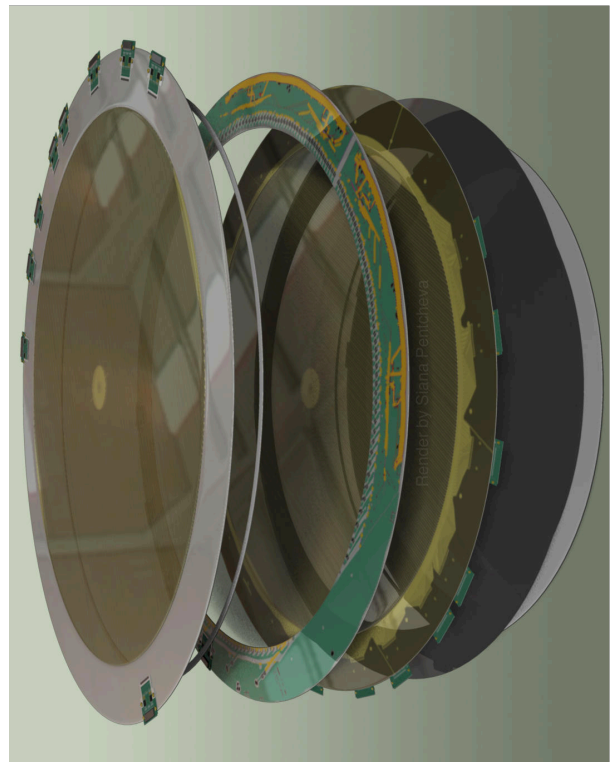

Figure 2.21: FDC cell view. Artistic view of one detector cell from left to right: upstream cathode, spacer ring, wire plane, downstream cathode, ground plane (black disk) glued to the back of the downstream cathode, end window (gray) only at the end of the package.

required in the forward tracking system. The FDC consists of 24 disk-shaped planar drift chambers of radius $0.5 \mathrm{~m}$ grouped in 4 packages. Six chambers are grouped together to form one of the four FDC packages. Figure 2.21, reproduced from ref. [35], shows an artistic view of one chamber of the FDC. Each chamber contains a wire plane with two cathode planes, each of which, is located on either side of the wire plane. The copper strips on the cathodes are arranged at an angle of $30^{\circ}$ relative to each other and at angles of $75^{\circ}$, and $105^{\circ}$ relative to the wires [37]. The read out of FDC detector contains the charge induced on the strips and the timing information from the wires. There are a total of 2304 wires and 10368 strips. The information from the strips and the wires is used to reconstruct the location of the track along the wire, as well as its location transverse to the wire (using the drift time) [35].

\subsection{Barrel Calorimeter}

The BCAL is the outermost detector inside the solenoidal magnetic field of the Gluex detector. Together with the FCAL it forms the Gluex calorimetry system. Both calorimeters 


\section{BCAL Geometry}

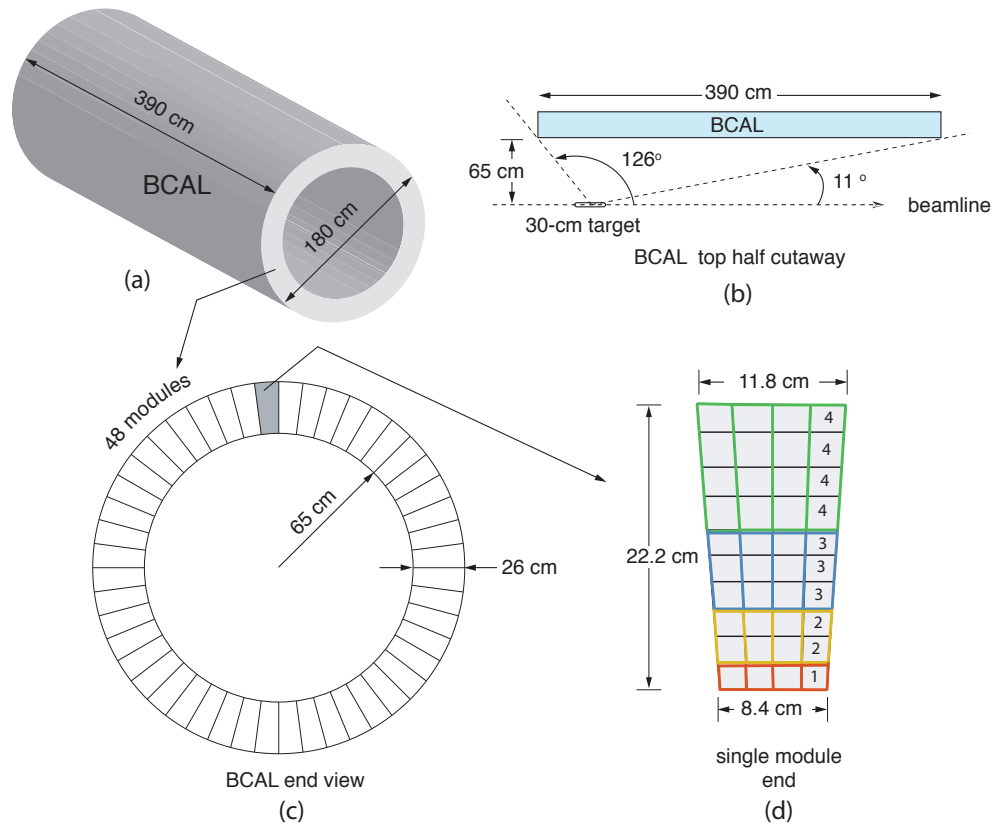

Figure 2.22: Sketch of the Barrel Calorimeter and readout. (a) A three-dimensional BCAL view; (b) top-half cutaway (partial side view) of a BCAL module showing its polar angle coverage and location with respect to the GlueX target; (c) end view of the BCAL depicting all 48 azimuthal modules and (d) wedge-shaped end view of a single module.

measure energies of photons resulting from the decays of $\pi^{0^{\prime}} s, \eta^{\prime} s$, and other radiative decay processes. The BCAL detector determines the energy and information of electromagnetic showers produced, as well as the corresponding timing information. It can also provide timing information for charged particles, allowing for additional time-of-flight data for particle identification. The BCAL is a lead scintillating fiber callorimeter. It consists of 48 optically isolated modules each with a trapezoidal cross section, forming a $390 \mathrm{~cm}$-long cylindrical shell with inner and outer radii of $65 \mathrm{~cm}$ and $90 \mathrm{~cm}$, respectively. The scintillating fibers run parallel to the cylindrical axis of the detector. Schematics showing the geometry of the BCAL and readout segmentation are shown in Fig. 2.22 from ref. [46]. The detected photon showers have an energy range between $0.05 \mathrm{GeV}$ and several $\mathrm{GeV}$. They cover a polar angle range between $11^{\circ}$ and $126^{\circ}$, for all the azimuthal angles. More details about the construction, and performance of the BCAL can be found in reference [46]. 
TOF Detector

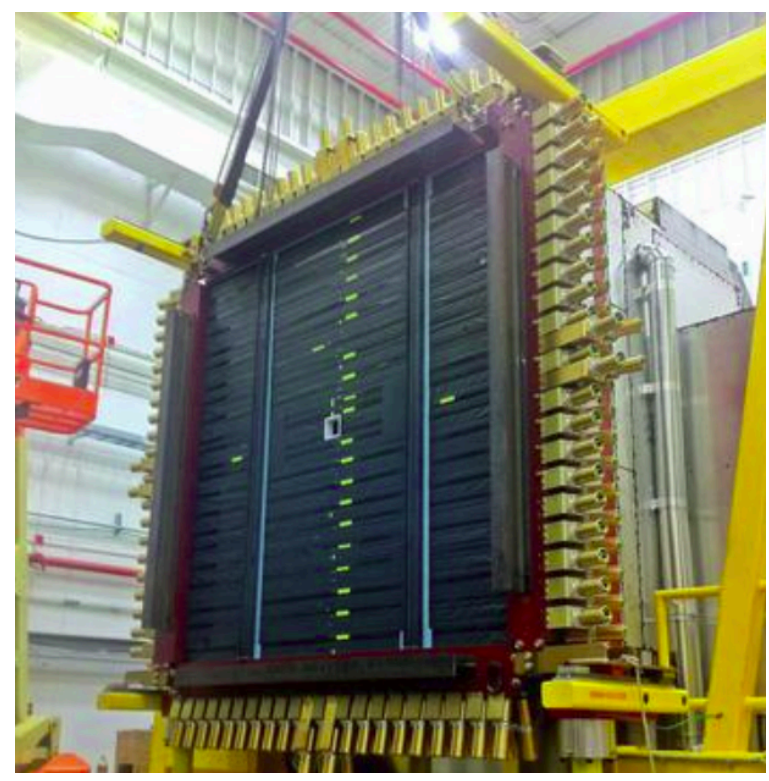

Figure 2.23: TOF wall on its support frame.

\subsection{Time of Flight}

The time of flight (TOF) detector, Fig. 2.23, is a large wall of scintillator bars located about $5.5 \mathrm{~m}$ downstream of the target. The scintillator bars are arranged in two planes where they are oriented horizontally in the first plane and vertically in the second one. The detector measures the flight time of charged particles leaving the target from the event vertex. It covers a polar angle range between $0.6^{\circ}$ and $13^{\circ}$ [37]. By combining the path length of the particle found using the tracking information with the timing information from the TOF, the particles velocities can be determined. One can then compare the expected flight time for a given mass hypothesis against the observed time-of-flight to reject misidentified particle hypotheses. Thus TOF detector provides important PID information.

\subsection{Forward Calorimeter}

The forward calorimeter (FCAL) consists of 2800 lead-glass blocks, each $4 \times 4 \times 45 \mathrm{~cm}^{3}$, stacked behind the Time-of-Flight detector in a circular pattern. In lead glass, electromagnetic showers produce Cherenkov radiation that is detected by photo-multipliers attached 


\section{Photon Resolution}
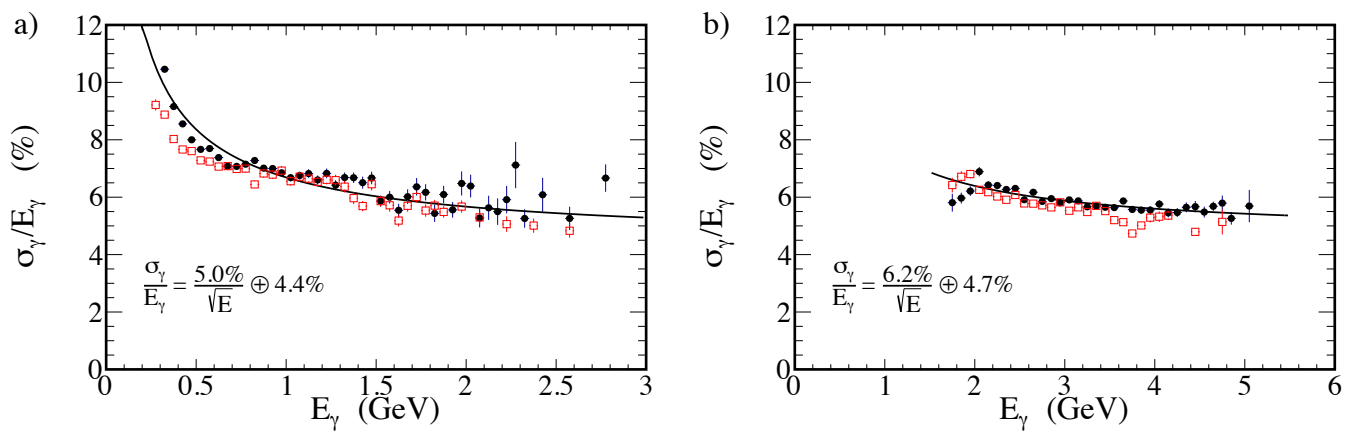

Figure 2.24: The energy resolution $\sigma_{\gamma} / E_{\gamma}$ for single photons in the a) BCAL and b)FCAL calculated from $\eta$ mass distribution with the assumption that only the energy resolution contribute to its width. Black solid circles are data, and red open squares are simulation.

to the back of each block. The showers typically spread over multiple blocks in the FCAL. The detected number of cherenkov photons is proportional to the energy deposited in the blocks, and therefore can be used to determine the total energy of the shower. By combining information from the individual detector blocks, the shower energy, position, and arrival time can be determined. Typical photon energies measured by the FCAL range from 0.1 $\mathrm{GeV}$ to several $\mathrm{GeV}$, and the polar angle coverage for the FCAL is bewteen $1^{\circ}$ and $11^{\circ}$. The single photon energy resolution $\sigma_{\gamma} / E_{\gamma}$ as a function of photon energy is shown in Fig. 2.11(from [37]) for the two calirometers. 


\section{CHAPTER 3}

\section{Event Selection for $\eta$ Photo-production for $2.9 \mathrm{GeV} \leq E_{\gamma} \leq 5.9 \mathrm{GeV}$}

In Fall 2018, the GlueX collaboration collected data for photon energies ranging from 2.9 to $5.9 \mathrm{GeV}$ during a short dedicated running period. The beam energy has been chosen to create a kinematic overlap with the CLAS $\eta$ photo-production experiments. The published CLAS results $[8,9]$, allow for a comparison with the new GlueX results in the same kinematic region. The Fall 2018 low energy dataset consists of 48 runs collected in Run Period 2018-08 between November 8 and November 12, 2018. For this data set the dipole magnet of the Pair Spectrometer was adjusted as described previously for photon flux measurements at the lower photon beam energies. The $\eta$ is identified by its decay into two photons. The reaction and decay products can be written as $\gamma+p \rightarrow \eta+p$, and $\eta \rightarrow \gamma \gamma$. The branching ratio for this $\eta$ decay according to the particle data group [47] is $39.41 \pm 0.20 \%$. All the final state particles were detected for this reaction.

In the first section of Chapter 3, the $\eta$ event selection will be discussed. The data Monte Carlo (MC) comparison will be presented in section 3.2 with a brief explanation of the simulation framework. The differential cross section determination is described in section 3.3 .

\subsection{Event Selection of $\eta$ Mesons}

The data were reconstructed using the standard Hall D reconstruction software, which produces Reconstructed Events STorage (REST) files denoted by version 2018-08_ver02. These REST files include information about all possible $\gamma p$ reactions. The standard analysis software frame work [48] allows one to subsequently select a specific reaction of interest. The $\gamma+p \rightarrow \eta+p$, and $\eta \rightarrow \gamma \gamma$ reactions have been extracted from the REST files using the Hall D analysis version 2018-08_ver05 and stored in ROOT trees [49]. The ROOT trees contain 4-vectors of all particle combinations of the reaction of interest. These combinations have to satisfy a set of minimal requirements such as convergence of the kinematic fitting procedure, requiring energy and momentum conservation, and a common production event 
BCAL $\Delta T$ cut

$\mathrm{p}$

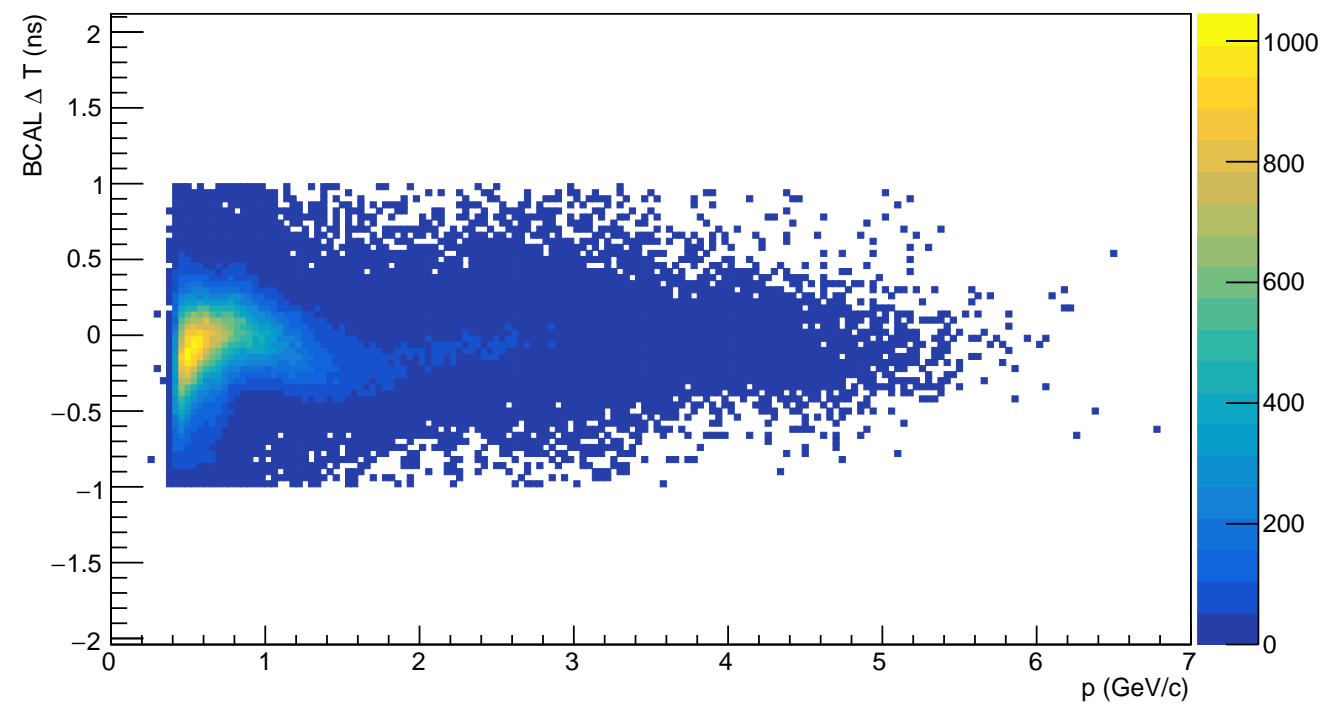

Figure 3.1: Proton identification timing cut in the BCAL

vertex. During kinematic fitting, the original measurements of the final state four vectors are adjusted within the detector resolution subject to constraints such as energy and momentum conservation and common vertex requirements and additional constraints. During the initial selection of the reactions, common predefined cuts for any GlueX analysis such as particle identification time difference cuts were applied as well. The common cuts are generally very wide, and are applied for all possible reactions. An example of a typical common cut is the BCAL $\Delta T$ cut for proton identification shown in Fig. 3.1, where $\Delta T$ refers to the time difference between the measured and calculated time for a proton candidate. A true proton will have a timing distribution centered around $\Delta T=0$ within the time resolution.

The produced trees were further analyzed using the DSelector class [50], derived from the more general ROOT TSelector class. In the DSelector analysis step, several custom cuts were applied that are specific to the reaction under study. For the current analysis two main cuts have been applied at the beginning of the DSelector code. The first is a beam energy cut between 2.9 and $5.9 \mathrm{GeV}$. The second is a broad missing mass $M_{M}^{2}=\left(p_{i}-p_{f}\right)^{2}$ cut, where $\left|M_{M}^{2}\right|<0.1 \mathrm{GeV}^{2}$, and $p_{i}-p_{f}$ is the difference between the initial and final state total 


\section{Prompt and Accidental Peaks}

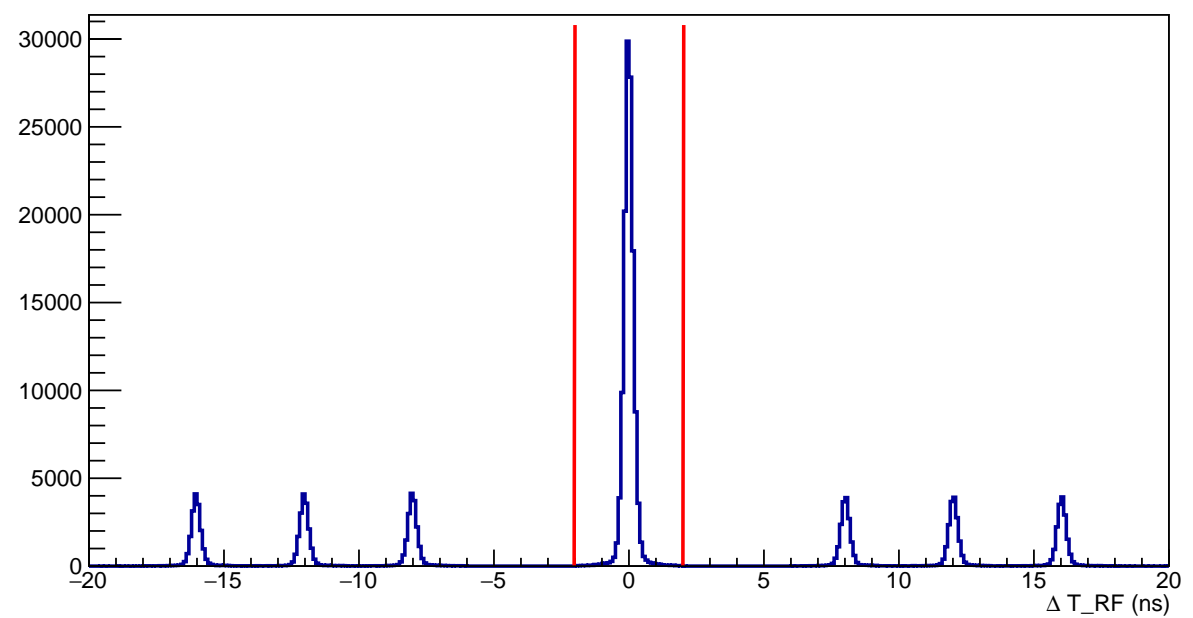

Figure 3.2: Time difference between tagged beam photons and RF time. The peak in between the red lines indicate the in-time beam photons. Outside these red lines show the out-of-time beam photons that will be used for accidental subtraction.

four momentum vectors. This difference is equivalent to the expression given by equation 3.1 .

$$
p_{i}-p_{f}=p_{\text {photon }}^{\text {beam }}+p_{\text {proton }}^{\text {target }}-\left(p_{\text {proton }}^{\text {recoil }}+p_{\gamma_{1}}+p_{\gamma_{2}}\right)
$$

where $p_{\text {photon }}^{\text {beam }}, p_{\text {proton }}^{\text {target }}, p_{\text {proton }}^{\text {recoil }}$ and $p_{\gamma_{1(2)}}$ are the 4-momenta of the tagged beam photon, target proton at rest, measured final state proton, and measured final-state photons, respectively. The time of the primary interaction is determined by a match between a SC hit and the proton track which identifies the RF bunch of the electron beam. Figure 3.2 shows the time difference $\delta t=t_{\text {beam }}-t_{R F}$ between the tagged-beam photon and the machine RF signal, where the region between the solid red lines indicates the signal region, consisting of a single RF bunch. Figure 3.2 also shows the out-of-time accidental tagged photons, which can be estimated with the following method.

The accidentally tagged photon contribution to the main peak distribution is estimated by selecting a sample of accidentally tagged combos with $6.012<|\delta t|<18.036$ ns. The accidental contribution has been subtracted by scaling the accidental sample with a 


\section{Kinematic Fitting $\chi^{2}$ cut}

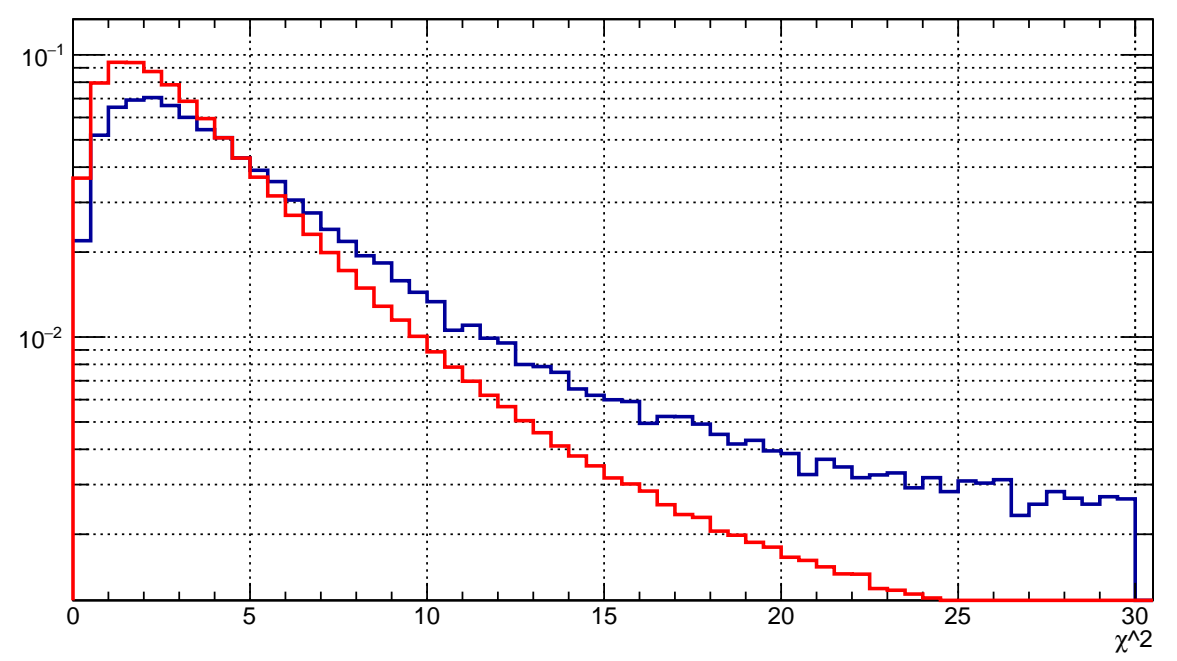

Figure 3.3: $\chi^{2}$ distributions scaling from kinematic fitting of data event (blue) and simulation events (red). A nominal cut is applied at $\chi^{2}=30$.

factor of $1 / 6$ to account for the number of bunches that were integrated over. Therefore each histogram was filled with a weight of $-1 / 6$ for events that are outside of the coincidence window (red lines) in Fig. 3.2. The events within the beam photons timing window have a weight $=1$.

The $\eta$ photo-production reaction was also simulated in a Monte-Carlo (MC) calculation. The theoretical $\eta / \eta^{\prime}$ cross section used in the event generation used a Regge based model by JM.Laget $[51,52]$. Since this model is based on t-channel exchange processes and

\section{Kinematic Fitting confidence level.}
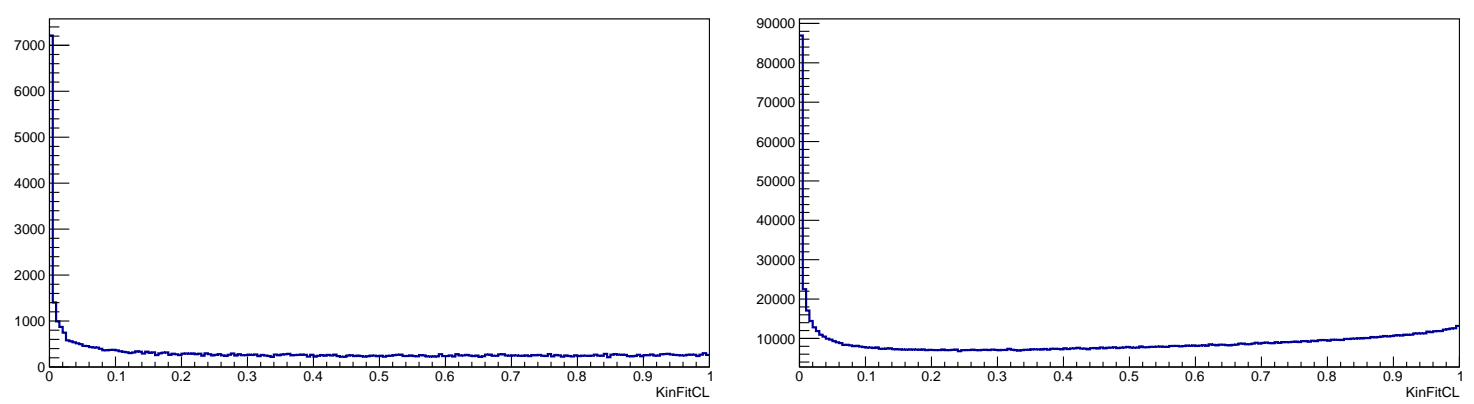

Figure 3.4: Kinematic fitting confidence level for data (left), and simulation (right) after $\chi^{2}$ cut has been applied. 


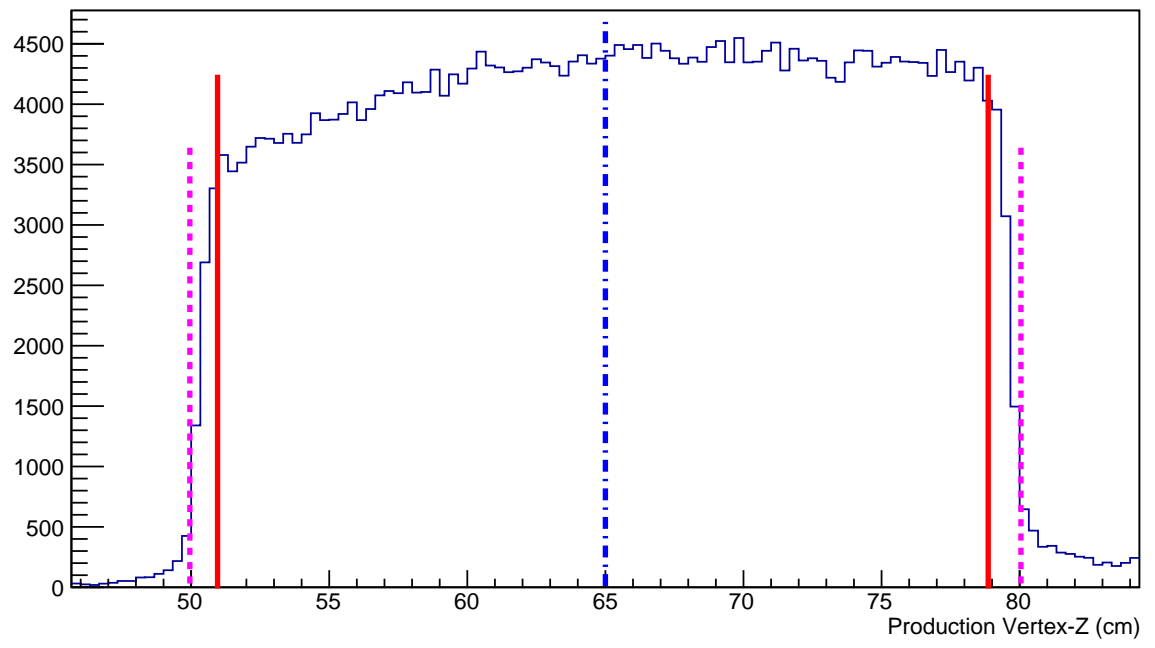

Figure 3.5: The production vertex $\mathrm{z}$ coordinate. The center of the target is located at $\mathrm{z}$ $=65 \mathrm{~cm}$ (dashed dotted blue). The selected events (between red lines) have their vertices within the target and are avoiding the target wall (magenta lines).

consequently most of the events were created in the forward direction, one also needs to create events using phase space based generator called genr8. This is essential to determine the acceptance for the photo-produced $\eta$ 's in the backward angles $\left(\theta_{c m}^{\eta}>90^{\circ}\right)$. In both cases, the detector response was simulated using the GEANT4 framework [53], which simulates the passage of particles through matter. More details about the GlueX reaction simulation and data comparison are discussed in section 3.2. The kinematic fit $\chi^{2}$ distributions for the measured and simulated data are normalized by each total area and are shown in Fig. 3.3, where a nominal $\chi^{2}=30$ cut has been applied. For $\chi^{2}>25$, the simulated data have zero counts, while the measured data have at least an order of magnitude less counts than its peak. The fall off in the data is slower than in the simulation because of the relatively more background in the data more than in the simulation. Another measure for the goodness of the kinematic fitting process is the confidence level where it shows a flat distribution in both data and simulation as in Fig. 3.4.

The $\gamma p \rightarrow p \eta$ event candidates were selected using the following cuts:

- $\Delta T=t_{\text {tagger }}-t_{R F}:-2.004<|\Delta t|<2.004$ ns. (Figure 3.2)

- Vertex cut: $R<1$, and $51.0<z<79.0$. (Figure 3.5) 


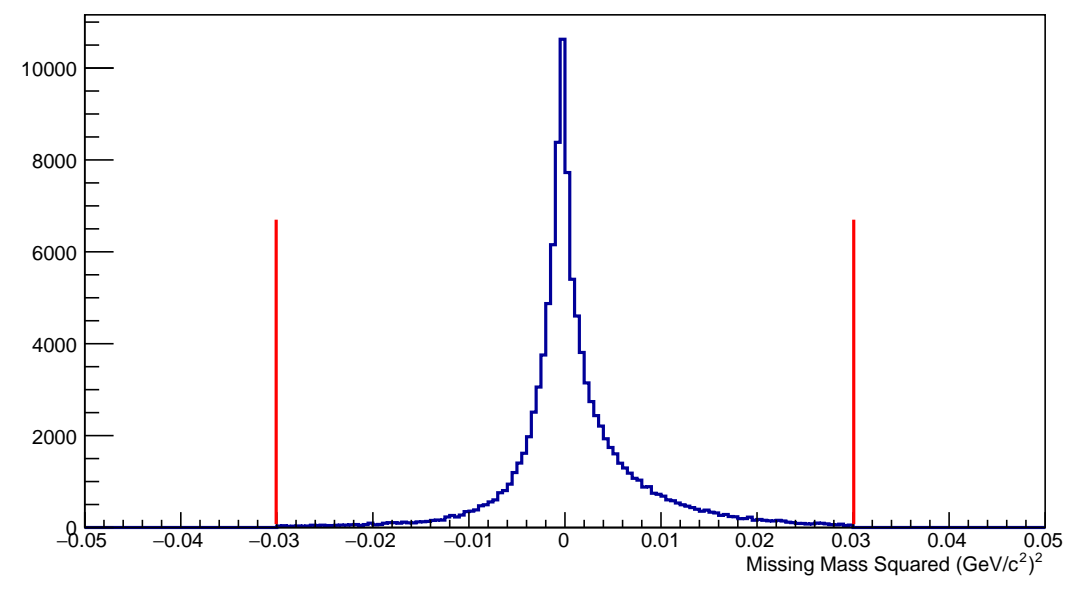

Figure 3.6: $\left|M_{M}\right|^{2}$ distribution with cut (red lines) .

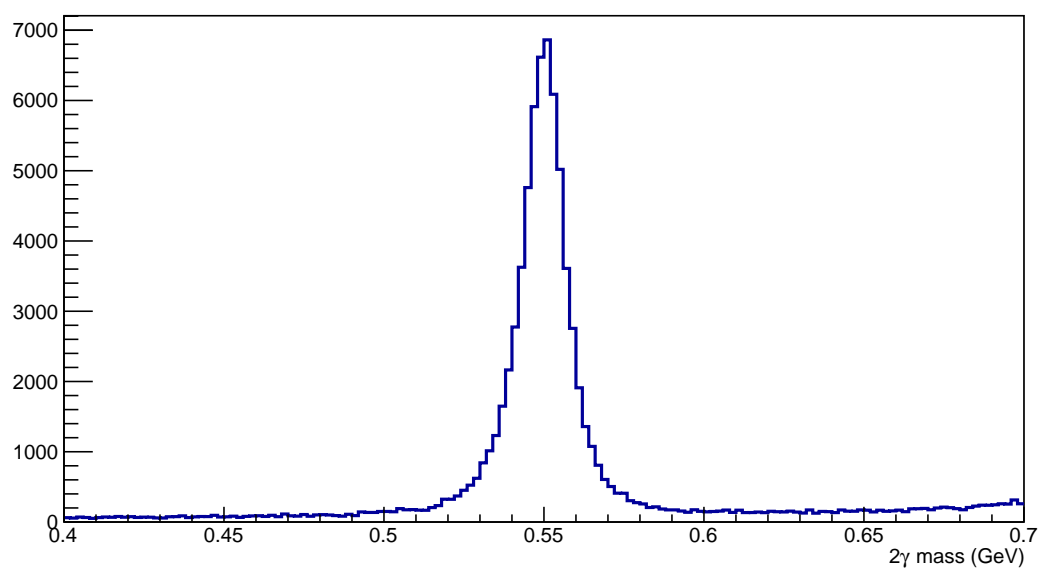

Figure 3.7: The $2 \gamma$ invariant mass spectrum for events passing all the selection cuts.

- Missing Mass: $\left|M_{M}^{2}\right|<0.03 \mathrm{GeV}^{2}$. (Figure. 3.6)

- Recoil proton momentum >0.350 GeV.(Figure. 3.10)

The vertex cut was applied to ensure that all the final state particles originated within the target itself and not from the walls. Figure 3.5 shows the production vertex z coordinate (along the photon beam) and the selected limits. Figure 3.6 show the missing mass squared $\left|M_{M}\right|^{2}$ distribution after its cut has been applied. A clear $\eta$ signal is obtained with a background increasing with increasing $2 \gamma$ invariant mass, as shown in Fig.3.7. This quite clean $\eta$ signal will be used to extract the $\eta$ yield and to determine differential cross sections. 


\subsection{Data Monte-Carlo comparisons}

During this analysis an event generator [52], called genEtaRegge, based on Regge modeled cross sections, is used to generate either $\eta$ or $\eta^{\prime}$ events. These events are created by selecting a beam energy range where the cross section is calculated according to the model by J.M. Laget [51]. The interaction between the photon beam and the proton target is described via the exchange of several particles (Regge trajectories). At beam energies away from the resonance region, $\eta$ photoproduction on a proton target is a t-channel process with vector mesons exchange. The coupling constant $\left(V_{\gamma \eta}\right)$ of the vector meson photon vertices has contibutions from $\rho, \omega$, and the non negligible branching ratio of $\phi$. At the low energy Gluex data set, $\eta$ mesons are also produced in a backward angles $\theta>90^{\circ}$. Therefore another generator is needed to calculate the acceptance for none t-channel production mechanisms (u-channel Regge trajectories). A simple phase space event generator [54], genr8, is used. In genr8 the differential cross section $d \sigma / d t$ is proportional to $e^{-b|t|}$, where $\mathrm{b}$ is a parameter determined by the user, and $|t|$ is the momentum transfer squared. A sample of $10 \mathrm{M}$ events was generated with $b=-0.1$, to have enough events in the backward angles to calculate the acceptance.

The response of the GlueX detector to the generated events (from genEtaRegge + genr8) has been simulated using GEANT4. The simulation uses the same geometry definitions and magnetic field that are used in the reconstruction of the measured events. The experiment geometry includes the photon beam line starting at the radiator and ending at the Hall D photon beam dump. Background events collected during the experiment using a random trigger are combined with $\mathrm{MC}$ signal events during the event smearing step. In the event smearing step corrections to simulated detector hits are applied to account for inefficiencies and the finite resolution of the GlueX detector. All detectors have been calibrated and their calibration constants and inefficiency information have been stored in the Calibration Constants Data Base (CCDB). The Run Conditions Data Base (RCDB) contains the experimental conditions during each run such as polarization direction, beam current, energy,...etc. Both CCDB and RCDB provide the needed information for the smearing step. 
After the event smearing step the simulated event data should be a realistic representation of a real reaction event. The smeared MC events are then reconstructed using the same software used for data reconstruction and the results are stored in simulation REST files. The same analysis steps are then performed for the reaction of interest producing ROOT trees with simulated data ready for the final, DSELECTOR based, physics analysis.

To determine cross sections, a reasonable agreement between real data and simulated data is required. Figure 3.8 show the comparison between data and simulation of $\eta$ polar angle in the lab frame, from genEtaRegge, for 12 center of mass energy (W) bins ranging from $2.52 \mathrm{GeV}$ to $3.35 \mathrm{GeV}$. The central values of $\mathrm{W}$ for these bins are listed in table 3.2. There is a very good agreement between data and simulation, for $W<3 G e V$, up to $\theta=25^{\circ}(\theta$ being the angle between the $\eta$ and the beam), where most of the events were occurring. The agreement starts to degrade for $W>3 \mathrm{GeV}$, and the number of recorded and generated events have also decreased by at least an order of magnitude. For large angles, the genEtaRegge MC sample has scarce statistics compared to data, hence the genra8 phase space generator is used to get the events needed for acceptance calculations. Figure 3.8, shows also that $\eta$ production is peaked at forward angles and is further increasing with increasing center of mass energy.

The agreement between data and $\mathrm{MC}$ were also checked for individual final state particles. The normalized polar angle (momentum) distributions of each particle in the lab frame are compared between data and MC. Figure 3.9 presents the final state proton polar angle distribution for data and simulation for all center of mass energy bins. This distribution peaks at a polar angle of $\sim 70^{\circ}$ in both data and simulation. The higher the center of mass energy, the steeper the increase in the measured recoil proton polar angle, which corresponds to the $\eta$ peaking at increasingly forward angles. There is a very good agreement between data and simulation for $W<2.81$ for mostly all angles. The comparison between data and simulation for the photo-produced $\eta$ momentum is shown in Fig. 3.11. In general there is a good agreement between data and simulation of the $\eta$ momentum distribution for momentum $\left(p_{\max }-2 \mathrm{GeV}<p_{\eta}<p_{\max } G e V\right)$, where $p_{\max }$ is the maximum $\eta$ momentum for this $\mathrm{W}$ bin. There are two peaks in the $\eta$ momentum 

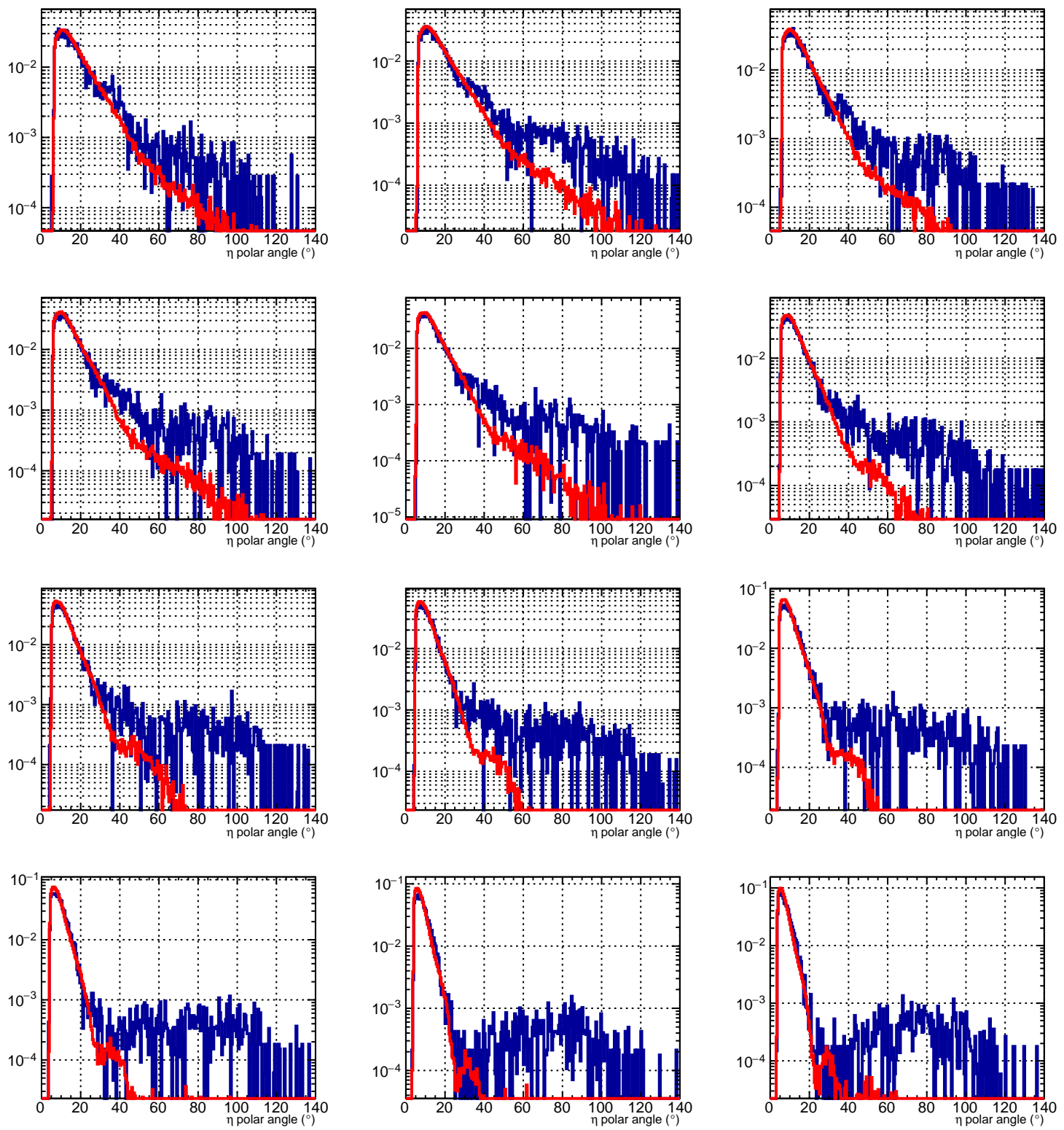

Figure 3.8: $\eta$ polar angular distribution comparison between data (blue) and simulation (red) in the lab frame for all center of mass energy bins, $\mathrm{W}=2.542 \mathrm{GeV}$ (top left) up to $\mathrm{W}=3.277 \mathrm{GeV}$ (bottom right). 

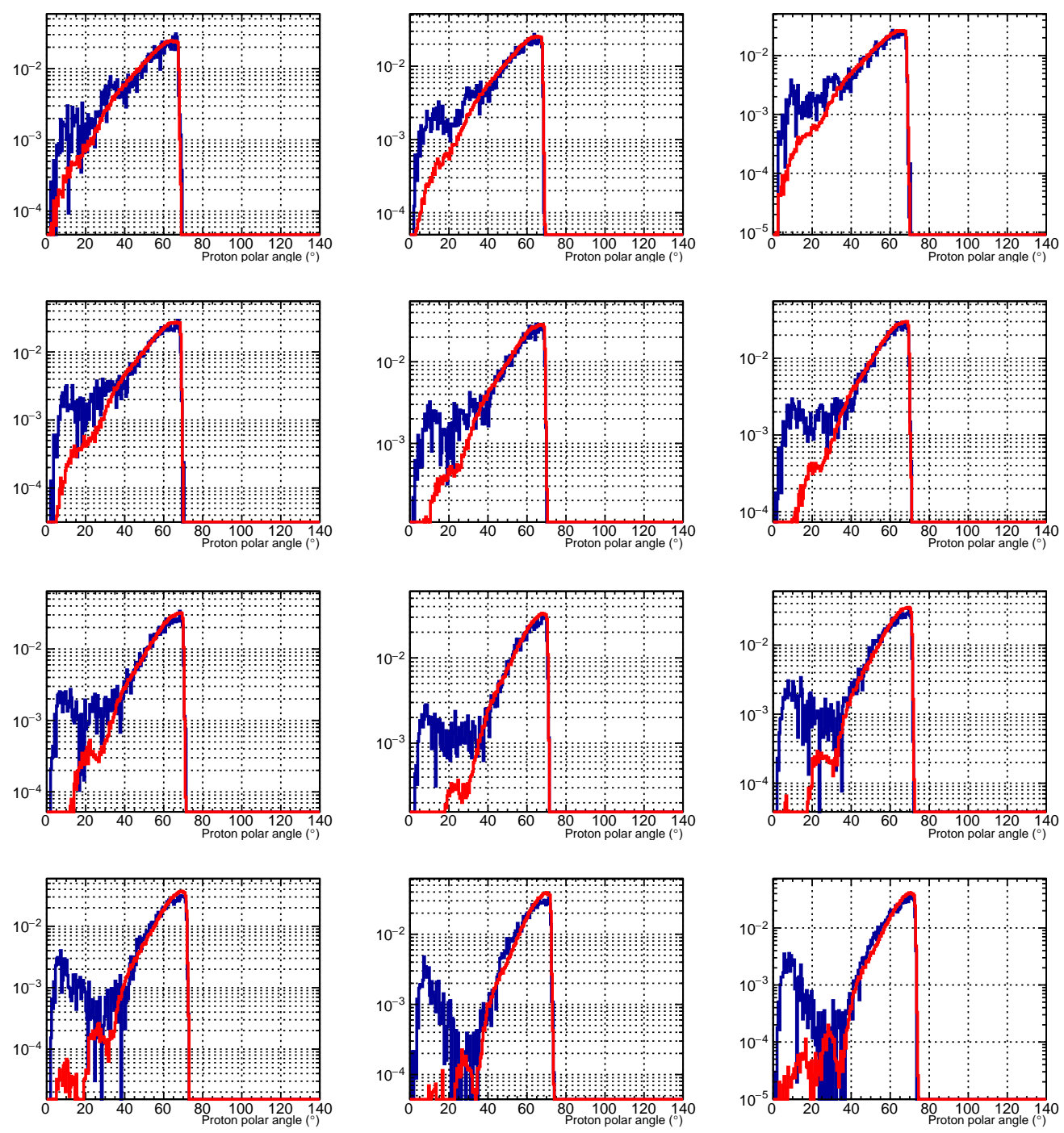

Figure 3.9: Recoil proton angular distribution comparison between data (blue) and simulation (red) in the lab frame for $\mathrm{W}=2.542 \mathrm{GeV}$ (top left) up to $\mathrm{W}=3.277 \mathrm{GeV}$ (bottom right). 

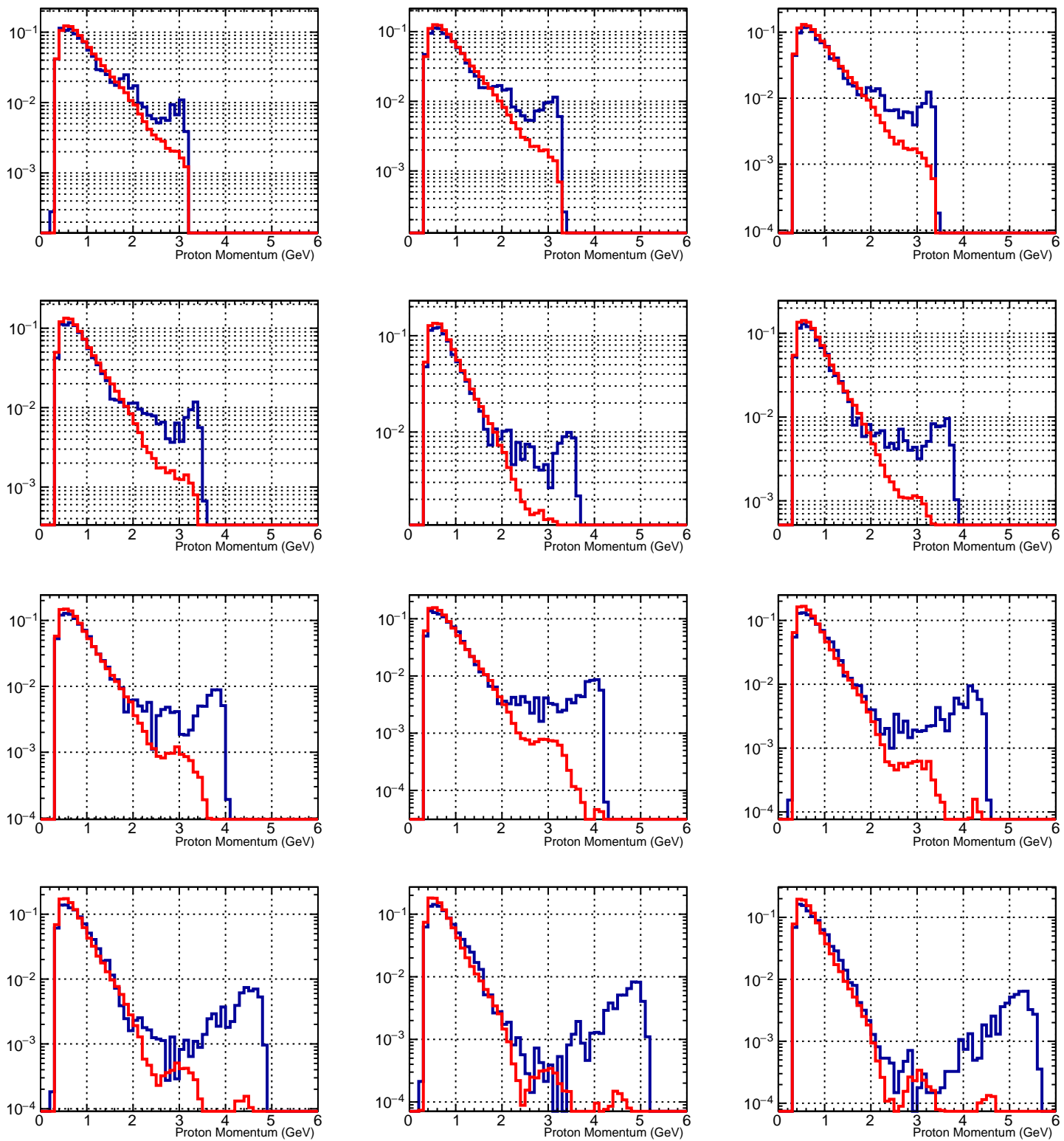

Figure 3.10: Recoil proton momentum distribution comparison between data (blue) and simulation (red) in the lab frame for $\mathrm{W}=2.542 \mathrm{GeV}$ (top left) up to $\mathrm{W}=3.277 \mathrm{GeV}$ (bottom right). 

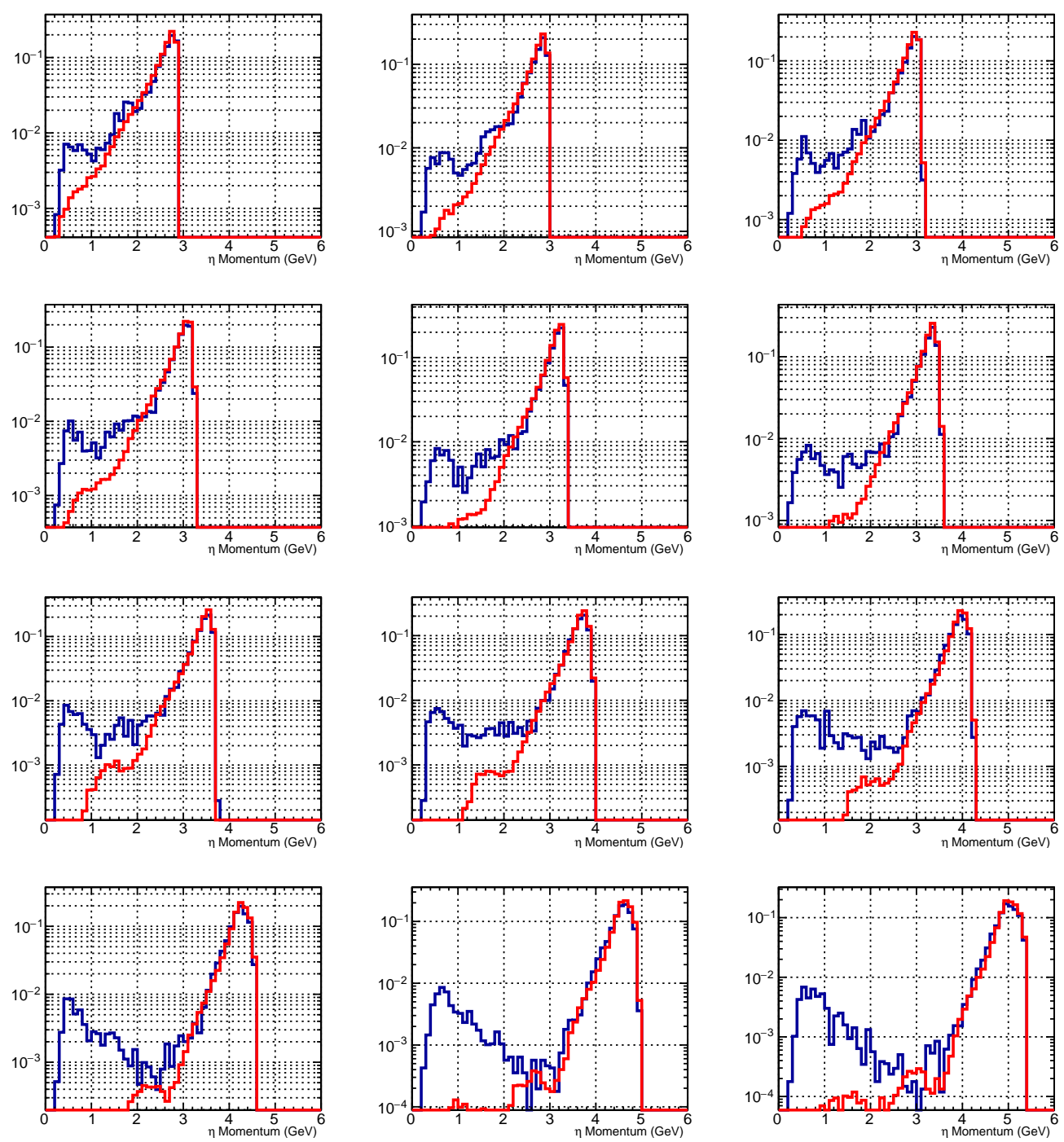

Figure 3.11: $\eta$ momentum distribution comparison between data (blue) and simulation (red) in the lab frame. 
distribution. The first peak is at a low momentum range with a nearly constant strength for all energy bins, probably indicating a u-channel production mechanism. The second peak is at the high momentum end and is at least an order of magnitude higher than the $\mathrm{u}$-channel peak. The high momentum peak corresponds to the forward angles, indicating the t-channel production mechanisms.

\subsection{Differential Cross Section Determination}

The differential cross section is extracted from the data using the following expression (eqn. $3.2)$.

$$
\frac{d \sigma}{d \Omega}=\frac{Y\left(W, \cos \left(\theta_{c m}^{\eta}\right)\right)}{A C C\left(W, \cos \left(\theta_{c m}^{\eta}\right)\right) N_{\gamma}} \frac{1}{\rho_{\text {target }}^{a}} \frac{1}{d \Omega} \frac{1}{B R}
$$

where:

- $Y\left(W, \cos \left(\theta_{c m}^{\eta}\right)\right)$ is the yield as a function of center of mass energy (W) and the production polar angle of $\eta$ in the center of mass frame $\cos \left(\theta_{c m}^{\eta}\right)$.

- $A C C\left(W, \cos \left(\theta_{c m}^{\eta}\right)\right)$ is the acceptance in each $\mathrm{W}$ and $\cos \left(\theta_{c m}^{\eta}\right)$ bins

- $N_{\gamma}$ is the number of incident photons in each $\mathrm{W}$ bin.

- $d \Omega=2 \pi \Delta \cos \left(\theta_{c m}^{\eta}\right)$

- BR is the branching fraction of the selected decay channel. In the case of $\eta \rightarrow \gamma \gamma$, $\mathrm{BR}=39.41 \pm 0.002 \%$.

- $\rho_{\text {target }}^{a}$ is the aerial target density, which is given by

$$
\rho_{\text {target }}^{a}=\frac{\rho_{T} L_{T} N_{A}}{A_{T}}
$$

where:

- $\rho_{T}$ is the target density

- $L_{T}$ is the target Length

- $N_{A}$ is Avogadro's number

- $A_{T}$ is the target atomic weight

The experimental cross section is determined as a function of the center of mass energy (W) and as a function of $\cos \left(\theta_{c m}^{\eta}\right)$, where $\theta_{c m}^{\eta}$ is the polar angle of the produced $\eta$ in the $\eta$-p center of mass frame. The $\eta^{\prime}$ differential cross section has been determined in the same way. 
$\eta$ Signal Fit
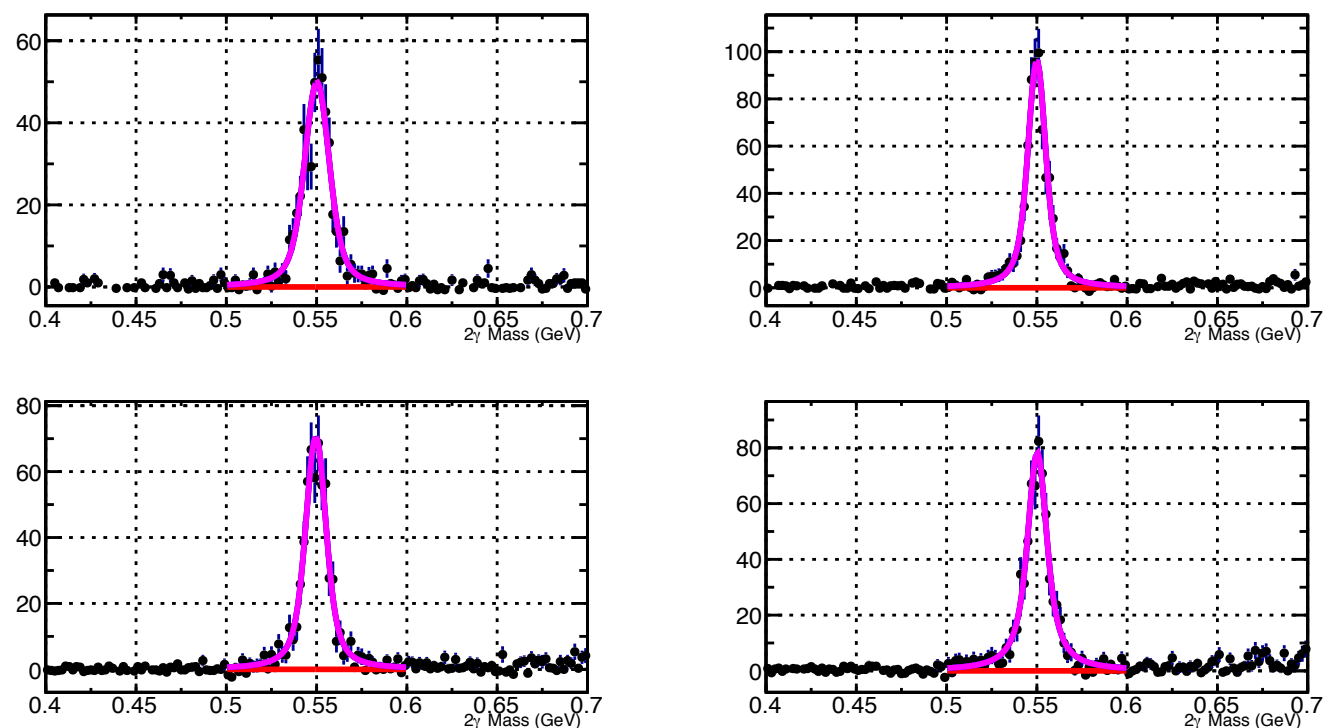

Figure 3.12: $\gamma \gamma$ invariant mass distribution fit to extract the yield for several bins at the center of mass energy $\mathrm{W}=3.053 \mathrm{GeV}$.

The constants used in equation 3.3 are shown in table 3.1. The center of mass energies are shown in table 3.2 as well as the corresponding beam energy values. The width of each bin $\left(\Delta \cos \left(\theta_{c m}^{\eta}\right)\right)$ is recorded in table 3.3 . The yield $Y\left(W, \cos \left(\theta_{c m}^{\eta}\right)\right)$ has been obtained by fitting a Voight function with a linear background to the $\gamma \gamma$ invariant mass spectrum for each center of mass energy $(\mathrm{W})$ and for each $\cos \left(\theta_{c m}^{\eta}\right)$ and integrating the fitted function over a $100 \mathrm{MeV}$ range, see Fig. 3.12. The fitted yield has been compared to the histogram sum over the same range, and deviation was less than $1 \%$. The yield is also calculated from the histogram sum with background subtraction from two side bands of equal width of $50 \mathrm{MeV}$ in both sides of the peak, with no significant deviation from the previous two methods. The incident photon $\left(N_{\gamma}\right)$ distribution as a function of $\mathrm{W}$ is shown in figure 3.13. In general the photon flux increases as $\mathrm{W}$ increases.

The acceptance has been calculated using the MC simulation described in section 3.2. The acceptance for each bin is given by the ratio between the reconstructed MC signal to the generated MC signal. The resulting acceptance for the twelve center of mass energy bins, calculated from the genEtaRegge MC sample, are shown in Fig. 3.14, and 3.15. This 


\begin{tabular}{|c|c|}
\hline variable & value \\
\hline$A_{T}$ & $1.00794 \mathrm{~g} / \mathrm{mole}$ \\
$\rho_{T}$ & $0.07092 \mathrm{~g} / \mathrm{cm}^{3}$ \\
$L_{T}$ & $28.0 \mathrm{~cm}$ \\
$N_{A}$ & $6.022 \times 10^{23}$ \\
\hline
\end{tabular}

Table 3.1: The value of constants used in equation 3.2.

\begin{tabular}{|c|c|}
\hline W center value $(\mathrm{GeV})$ & E center value $(\mathrm{GeV})$ \\
\hline 2.542 & 2.98 \\
2.580 & 3.08 \\
2.620 & 3.19 \\
2.660 & 3.30 \\
2.700 & 3.42 \\
2.750 & 3.56 \\
2.810 & 3.74 \\
2.875 & 3.94 \\
2.955 & 4.19 \\
3.053 & 4.50 \\
3.155 & 4.84 \\
3.277 & 5.26 \\
\hline
\end{tabular}

Table 3.2: The central values of each of the center of mass energy bins and the corresponding beam energy.

thesis concentrates on the t-channel processes and therefore the relatively large acceptance errors of the backward angles are not concerning. The general pattern for the acceptance as a function of $\cos \theta_{c m}^{\eta}$ is nearly similar for all bins with $W<2.875 \mathrm{GeV}$. Starting at the backward angles, the acceptance increases to reach a steady maximum of $\sim 65 \%$ at about $\cos \theta_{c m}^{\eta}>0.1$ then decreases gradually starting from $\cos \theta_{c m}^{\eta}>0.92$ up to the most forward point, where the acceptance decreases by about $10 \%$ from the maximum. The value of $\cos \theta_{c m}^{\eta}$ at the most forward point changes with the energy from about 0.94 up to 0.975 , because more forward bins start to populate with increasing $\mathrm{W}$ in agreement with the kinematics of the reaction. For $\mathrm{W} \geq 2.955 \mathrm{GeV}$, the backward angles have much less statistics than the forward angles. 
Incident Photon Flux Distribution

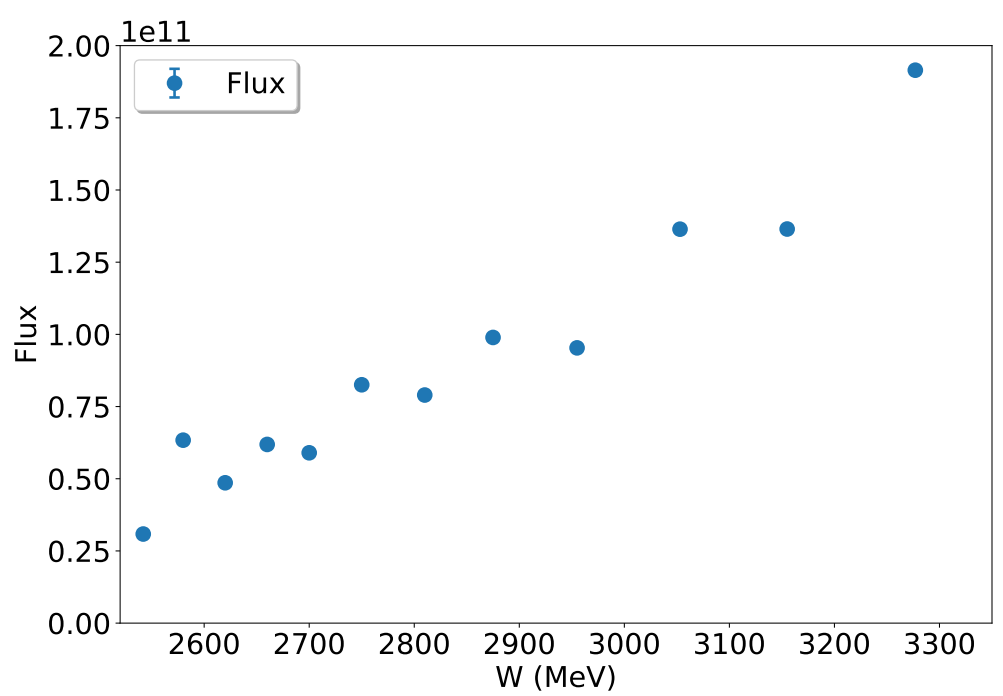

Figure 3.13: Photon flux at each of the center of mass energy bin

\begin{tabular}{|c|c|c|}
\hline Point number & Bin Start & Bin Width \\
\hline 1 & -1.00 & 0.19 \\
2 & -0.81 & 0.20 \\
3 & -0.61 & 0.30 \\
4 & -0.31 & 0.30 \\
5 & -0.01 & 0.20 \\
6 & 0.19 & 0.20 \\
7 & 0.39 & 0.10 \\
8 & 0.49 & 0.10 \\
9 & 0.59 & 0.10 \\
10 & 0.69 & 0.10 \\
11 & 0.79 & 0.04 \\
12 & 0.83 & 0.04 \\
13 & 0.87 & 0.04 \\
14 & 0.91 & 0.02 \\
15 & 0.93 & 0.02 \\
16 & 0.95 & 0.01 \\
17 & 0.96 & 0.01 \\
18 & 0.97 & 0.01 \\
\hline
\end{tabular}

Table 3.3: The beginning of the $\cos (\theta)$ bin and its width. 


\section{Acceptance.}
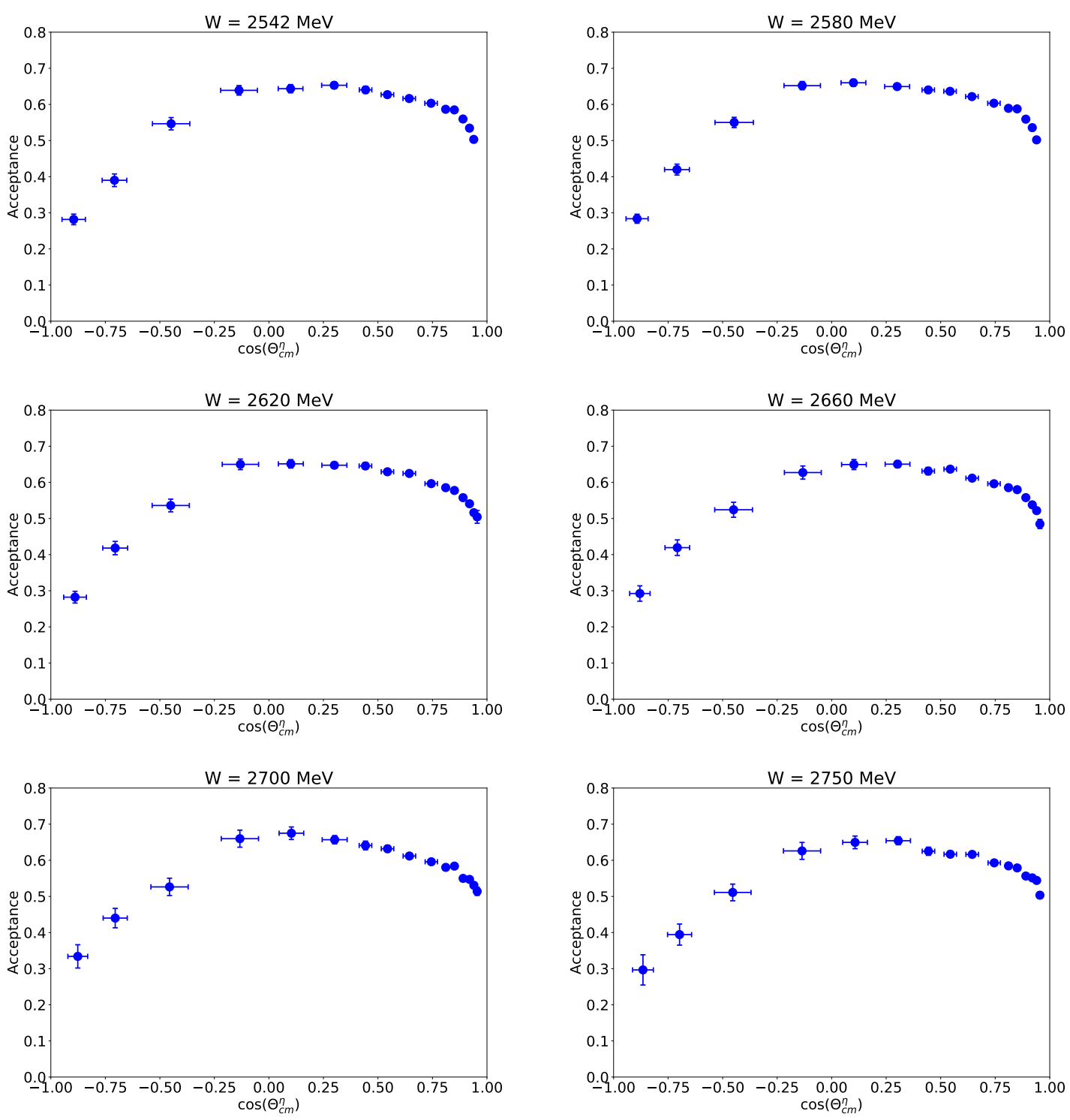

Figure 3.14: Acceptance as a function of $\cos \left(\theta_{c m}^{\eta}\right)$ from $\mathrm{W}=2.542$ up to $2.750 \mathrm{GeV}$. 


\section{Acceptance.}
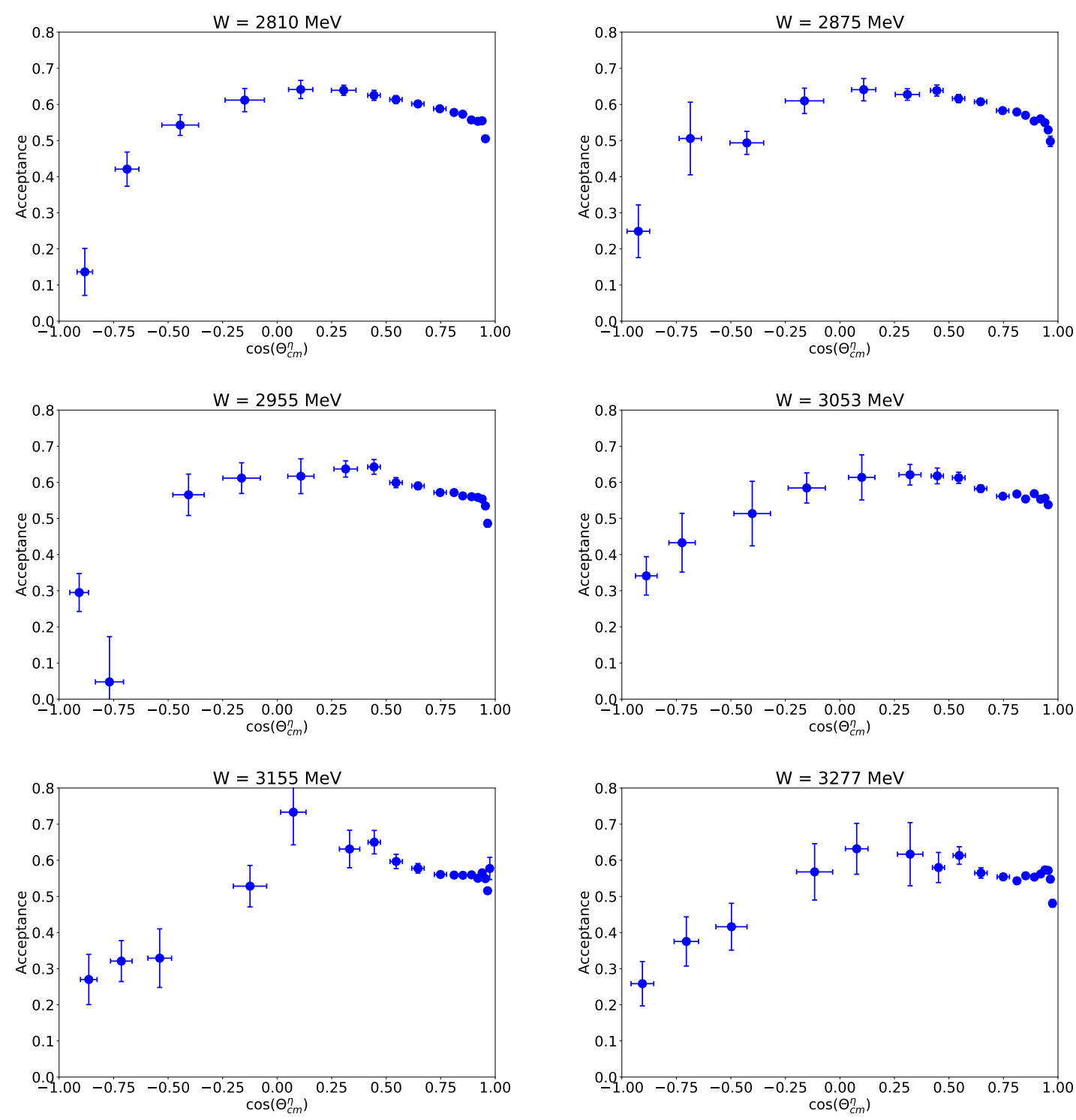

Figure 3.15: Acceptance as a function of $\cos \left(\theta_{c m}^{\eta}\right)$ from $\mathrm{W}=2.810$ up to $3.277 \mathrm{GeV}$. 


\section{CHAPTER 4}

\section{Differential Cross Sections For $\eta$-Photo-production At Low Beam Energies}

\section{$2.9 \mathrm{GeV} \leq E_{\gamma} \leq 5.9 \mathrm{GeV}$}

In this chapter the results of the differential cross sections $d \sigma / d \Omega$, and $d \sigma / d t$ are presented as well as a systematic error study.

\subsection{Differential Cross Section $(\mathrm{d} \sigma / \mathrm{d} \Omega)$ Results}

The differential cross section for $\eta$ photoproduction has been determined for twelve center of mass energy bins ranging from $2.542 \mathrm{GeV}$ to $3.277 \mathrm{GeV}$. The cross section results from this work are compared to published CLAS results [8,9], EtaMAID2018 theoretical calculations [11], and a Regge model by the Joint Physics Analysis Center (JPAC) [55,56]. In the EtaMAID2018 model, $\eta$ photoproduction contains contributions of $21 \mathrm{~N}^{*}$ resonance states in addition to a Regge-cut model background [10,11]. In the Regge model the $\eta$ differential cross section is computed with Regge amplitudes and the t-dependence is determined using finite energy sum rules $[55,56]$.

Fig. $\quad 4.1$ shows the differential cross sections $(d \sigma / d \Omega)$ as a function of $\left(\cos \theta_{c m}^{\eta}\right)$ in comparison with the published data [8,9], EtaMAID2018 theoretical predictions [11], and the model by V. Mathieu [55], in both linear and log scale plots to capture the comparison details over the full range of $-1.0<\cos \theta_{c m}^{\eta}<1.0$. The GlueX data are covering a larger $\theta_{c m}^{\eta}$ angle range than the CLAS data in both forward and backward directions. Overall the GlueX results are systematically larger than the previously published CLAS data by about 50\%, and are also larger than the EtaMAID2018 calculations. Calculations by V.M. based on the Regge model predictions agree with the GlueX experimental results for small polar angles where $\cos \theta_{c m}^{\eta}>0.80$. The new data roughly follow the shape of the EtaMAID2018 model specially for $\cos \theta_{c m}^{\eta}<0.80$ as shown in Figs 4.1, and 4.2. The experimental results from both GlueX and CLAS2020 are in a good agreement for backward angles range of $-0.8<\cos \theta_{c m}^{\eta}<-0.3$. 
Cross Section Results For $\mathrm{w}=2542 \mathrm{MeV}$ up to $2620 \mathrm{MeV}$.
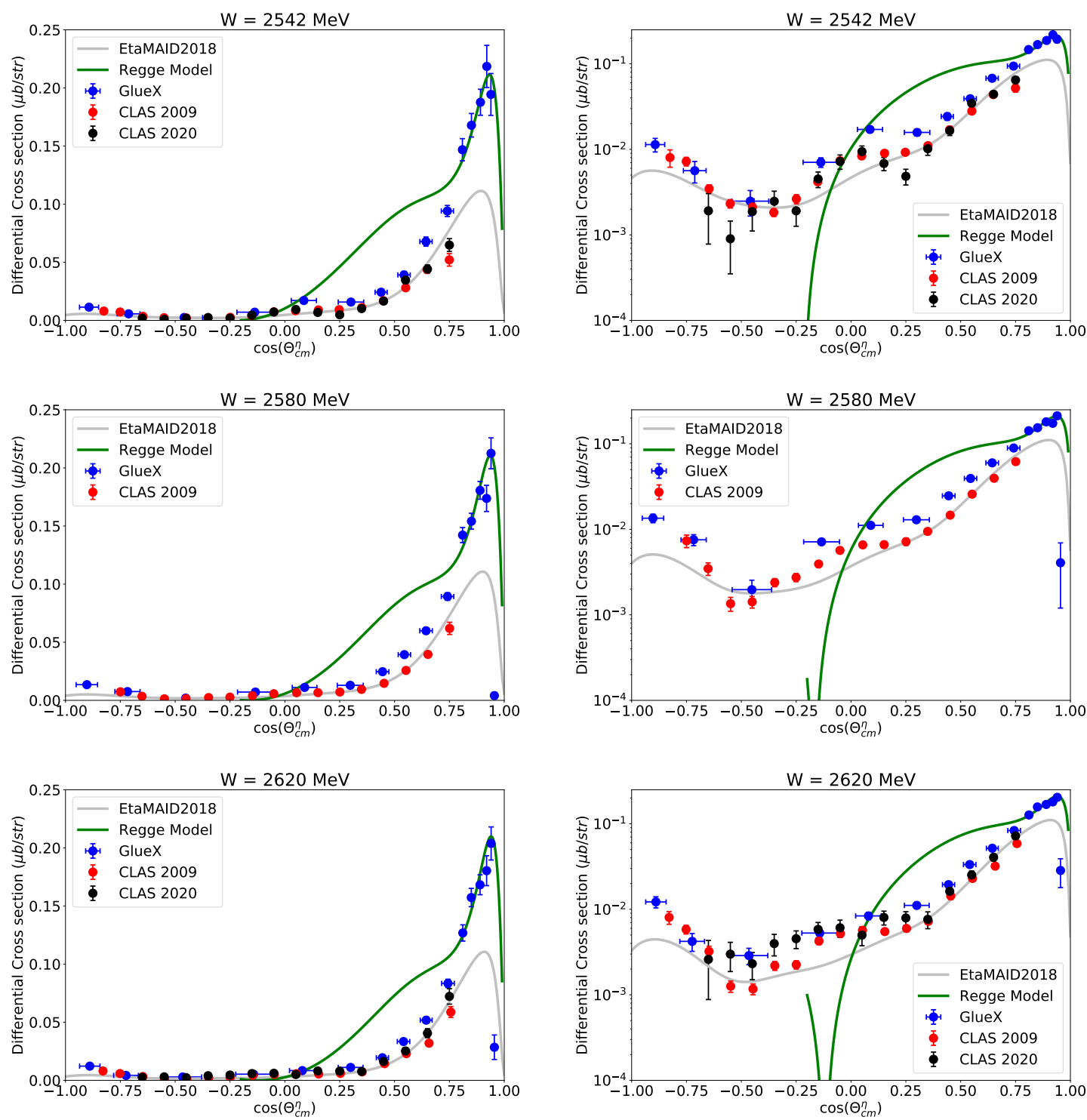

Figure 4.1: Differential cross section results in both linear (left), and log scale (right) for $\mathrm{W}=2542,2580$, and $2620 \mathrm{MeV}$. 
Cross Section Results for $\mathrm{w}=2660 \mathrm{MeV}$ up to $2750 \mathrm{MeV}$.
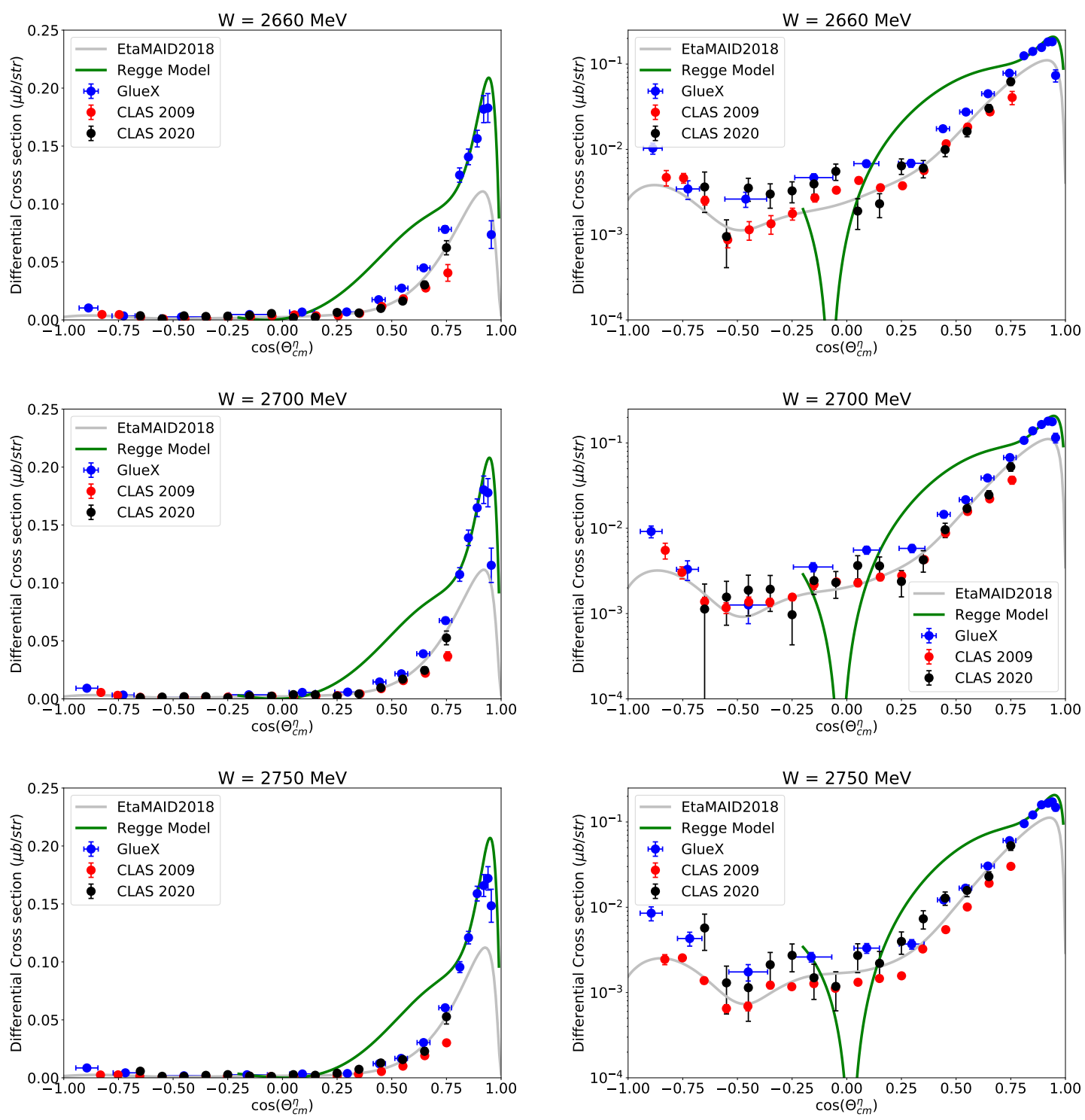

Figure 4.2: Same as Fig. 4.1, but $\mathrm{W}=2660,2700$, and $2750 \mathrm{MeV}$. 
In general, the EtaMAID2018 model predictions for the differential cross sections are much smaller than the GlueX data especially for small angles. The agreement between the EtaMAID2018 and this work results improves with increasing center of mass energy W and for large angles, where $\cos \theta_{c m}^{\eta}<0.3$. Therefore on average the EtaMAID2018 model agrees better with the data for large angles, especially for large W, while the Regge model agrees better at very small angles. Fig. 4.2 shows $d \sigma / d \Omega$ results for $\mathrm{W}=2660 \mathrm{MeV}$ up to 2750 $\mathrm{MeV}$. As $\mathrm{W}$ increases above $2700 \mathrm{MeV}$, the Regge model is overestimating the differential cross section while the EtaMAID2018 model is underestimating it for $\cos \theta_{c m}^{\eta}>0.9$. The differences between the new data and the EtaMAID2018 will be presented in more details in the next section discussing the $d \sigma / d t$ results.

Figs. $\quad 4.3$ shows the differential cross section results for center of mass energies $2.8<W<3.0 \mathrm{GeV}$, and Fig. and 4.4 for $3.0<W<3.35 \mathrm{GeV}$. The overlap between the CLAS2020 data and the current work extends up to $3155 \mathrm{MeV}$. There is a good agreement between the new GlueX data and the new CLAS data. These data cover an energy region above the production threshold $(\mathrm{W}=1.485 \mathrm{GeV})$ by more than $1.5 \mathrm{GeV}$ and extend beyond the resonance region. The threshold and the resonance region have been extensively studied during the last two decades $[2-7]$. At $\mathrm{W}=2875 \mathrm{GeV}$, the cross section does not vary very much over a large range of angles $\left(-0.7<\cos \theta_{c m}^{\eta}<0.2\right)$. The rise of the cross section at the backward angle is the results of u-channel production mechanisms. For center of mass energies of $W \geq 3.155 \mathrm{GeV}$, the production mechanism seems to be increasingly dominated by the t-channel processes in the forward direction $\left(\cos \theta_{c m}^{\eta}>0.7\right)$. At very small angles $\left(\cos \theta_{c m}^{\eta}>0.9\right)$, and $W \geqslant 3.155 \mathrm{GeV}$, the experimental differential cross section are increasingly over predicted by the Regge model and under predicted by the EtaMAID2018. At $\mathrm{W}>2.9 \mathrm{GeV}$ where the corresponding beam energy is greater than $4.7 \mathrm{GeV}$, the GlueX differential cross sections data are the most precise data currently available.

\subsection{Differential Cross Section (d $\sigma / \mathrm{dt})$ Results}

The differential cross section $(d \sigma / d \Omega)$, presented in the previous section, can be expressed as a function of the momentum transfer (-t) using the transformation shown in section 
Cross Section Results for $\mathrm{w}=2810 \mathrm{MeV}$ up to $2955 \mathrm{MeV}$.
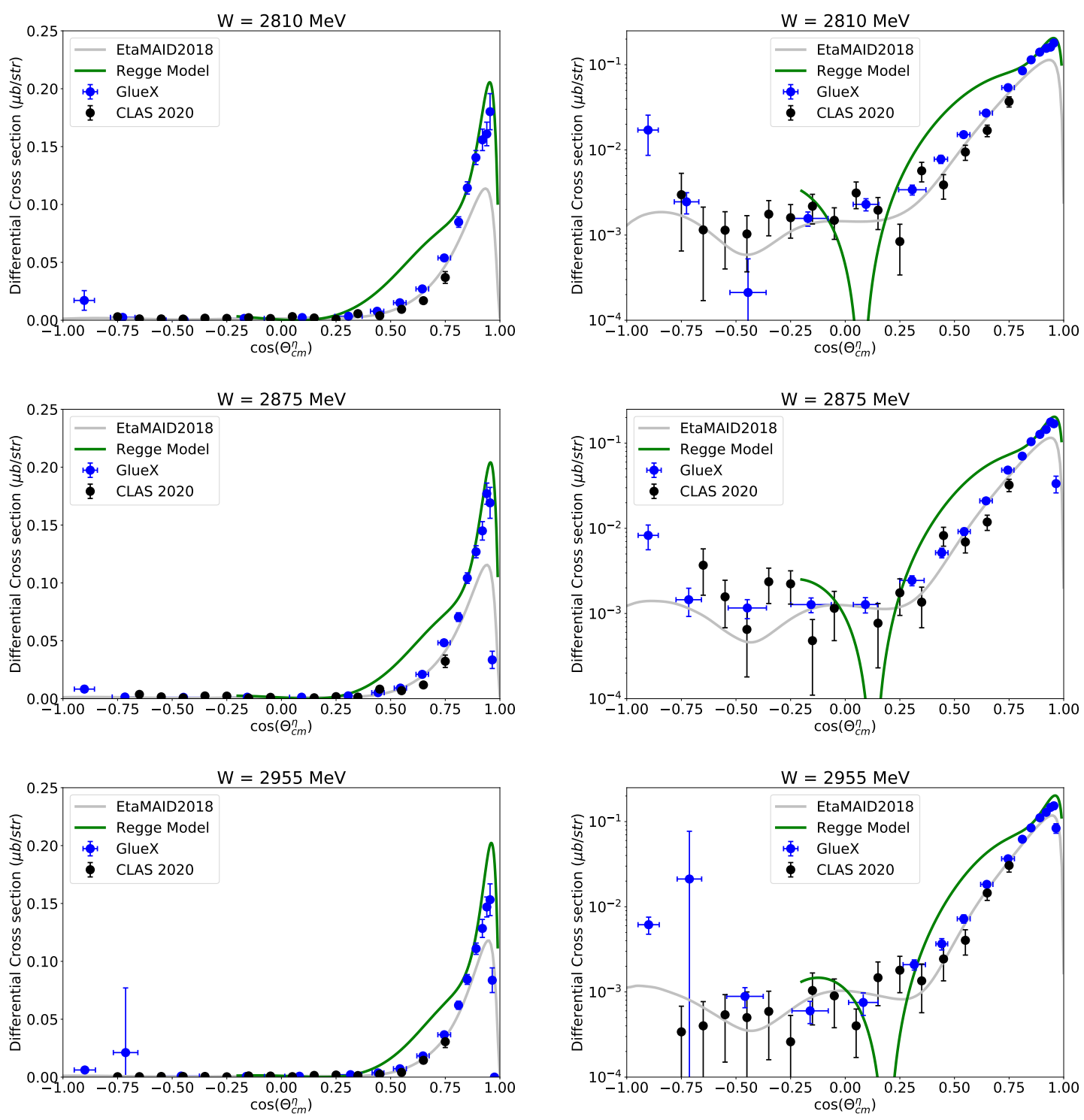

Figure 4.3: Same as Fig. 4.1, but $\mathrm{W}=2810,2875$, and $2955 \mathrm{MeV}$. 
Cross Section Results for $\mathrm{w}=3053 \mathrm{MeV}$ up to $3277 \mathrm{MeV}$.
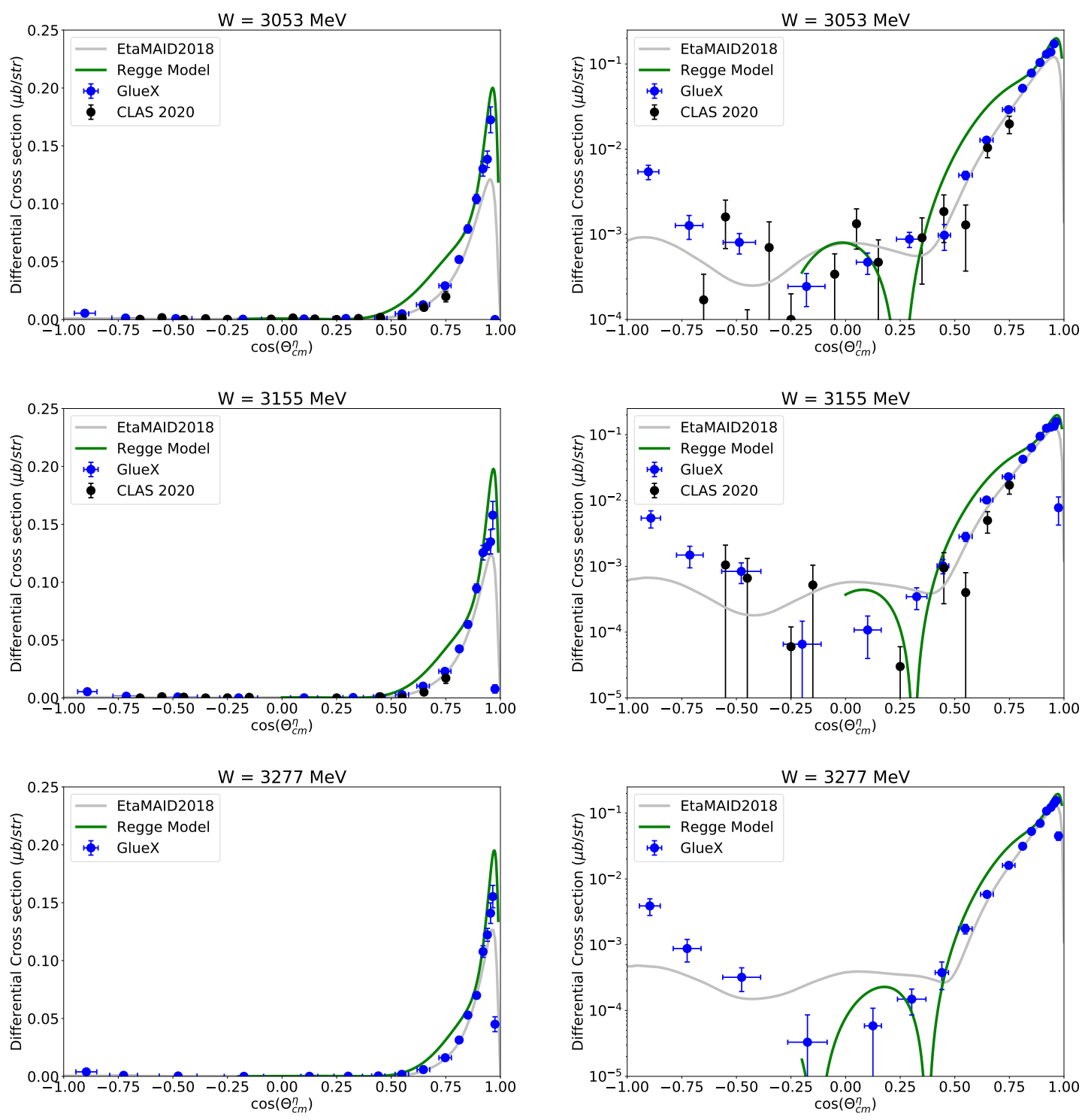

Figure 4.4: Same as in Fig. 4.1, but for $\mathrm{W}=3.053$ up to $3.277 \mathrm{GeV}$. 
1.4. Differential cross sections $d \sigma / d t$ are of great interest at beam energies $E_{\gamma}>4 \mathrm{GeV}$ in order to test Regge based models. The maximum available center of mass energy for the low energy data is $3.36 \mathrm{GeV}$. For the high energy data that will be discussed in the next chapter, the minimum available center of mass energy at $E_{\gamma}=6.2 \mathrm{GeV}$ is $3.54 \mathrm{GeV}$.

The differential cross section of photo-produced $\eta$ mesons at low energy as a function of $(-\mathrm{t})$ is presented in Fig. 4.5, and 4.6. A theoretical model prediction [51,52] by J.M. Laget is added for comparison with the data. For $-t<0.5 \mathrm{GeV}^{2}$, The Laget model is overestimating the cross section by about $20 \%$. The GlueX data provides differential cross sections for high (-t) values up to $8.5 \mathrm{GeV}^{2}$ at $\mathrm{W}=3.277 \mathrm{GeV}$ as well as for very small (-t) less than $0.2 \mathrm{GeV}^{2}$. For $0.5<-t<2.5 \mathrm{GeV}^{2}$, the GlueX measurements are in the middle between the Laget model and the EtaMAID2018 calculation. It is important to note that our data have not been used for parameter optimization in either model calculations. An improved agreement with the models can be expected when the GlueX data are taken into consideration. The Laget Model,on which the genEtaRegge generator is based on, has the same shape as the data $(0.9<-t<2.5)$. For $\mathrm{W}$ bins greater than $2810 \mathrm{MeV}$, the $\eta$ production peaks in the forward direction at very small values of (-t), typically at (-t $\left.<0.4 \mathrm{GeV}^{2}\right)$. At the highest energy bin $(\mathrm{W}=3.277 \mathrm{GeV})$, the GlueX data agree with the EtaMAID2018 model in the peak region, while agree better with the Regge model at lower energies as shown in Fig. 4.6.

Figs 4.7, and 4.8 show the ratio between the experimental results from CLAS2020 (C), GlueX (G) and the EtaMAID2018 (M) model. The ratio of $d \sigma / d t$ for each of the data sets are plotted with respect to the EtaMAID2018. The weighted average is then calculated for each data set. For $\mathrm{W}<2750 \mathrm{MeV}$, the GlueX data is on average higher than the EtaMAID2018 by about 50\%. The CLAS2020 results are also higher than EtaMAID2020 in some cases by about $60 \%$. The weighted average of $G / M$ is almost the same as $C / M$ at $\mathrm{W}=2620 \mathrm{MeV}$, and $2750 \mathrm{MeV}$. As stated before, EtaMAID2018 agrees well within 15\% of the GlueX data for $\mathrm{W}$ bins greater than $2955 \mathrm{MeV}$, and specifically in t-range of $0.2<$ $-t<2.0 G e V^{2}$. 
Differential Cross Section as a function of $\mathbf{- t}, 2.52<W<2.78 \mathrm{GeV}$.
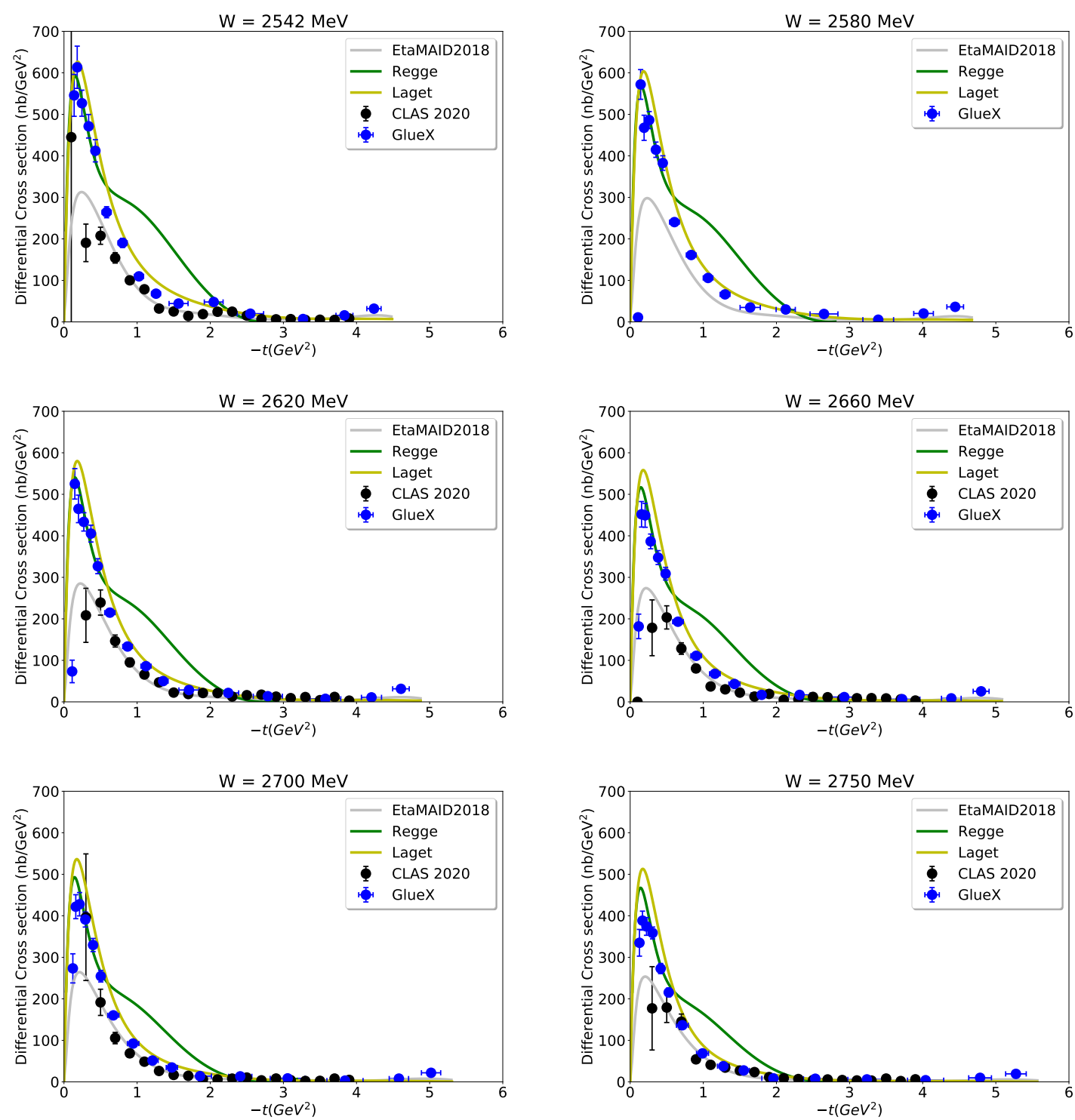

Figure 4.5: Differential cross section of $\eta$ mesons in a linear scale as a function of $-\mathrm{t} . \mathrm{W}=$ $2.542 \mathrm{GeV}$ up to $\mathrm{W}=2.750 \mathrm{GeV}$. 
Differential Cross Section as a function of $\mathbf{- t}, 2.78<W<3.36 \mathrm{GeV}$.
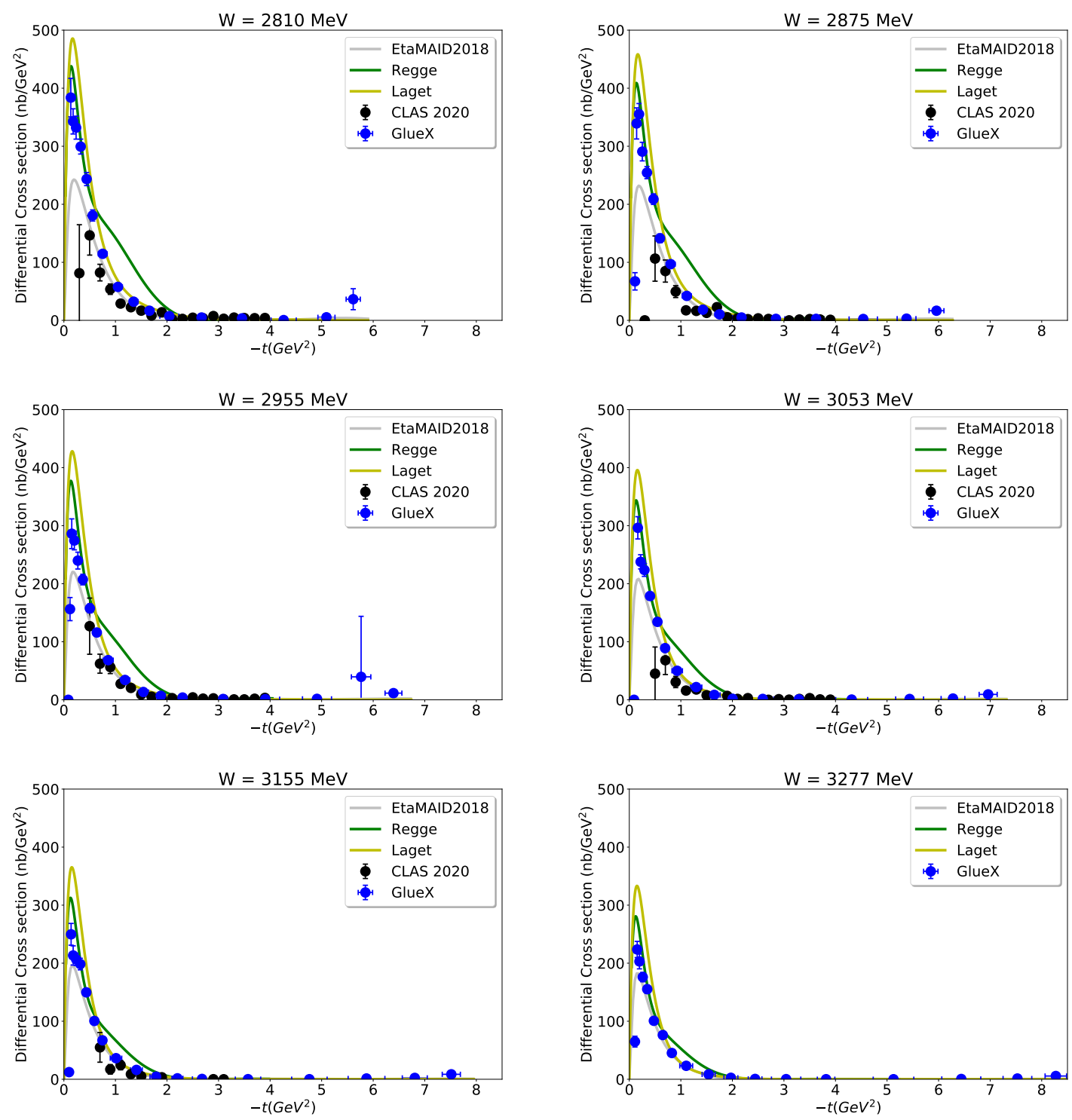

Figure 4.6: Same as 4.5 but for $\mathrm{W}=2.810 \mathrm{GeV}$ up to $\mathrm{W}=3.277 \mathrm{GeV}$. 
Weighted Average Differential Cross Section Ratios, W=2542 MeV up to $\mathrm{W}=2750 \mathrm{MeV}$.
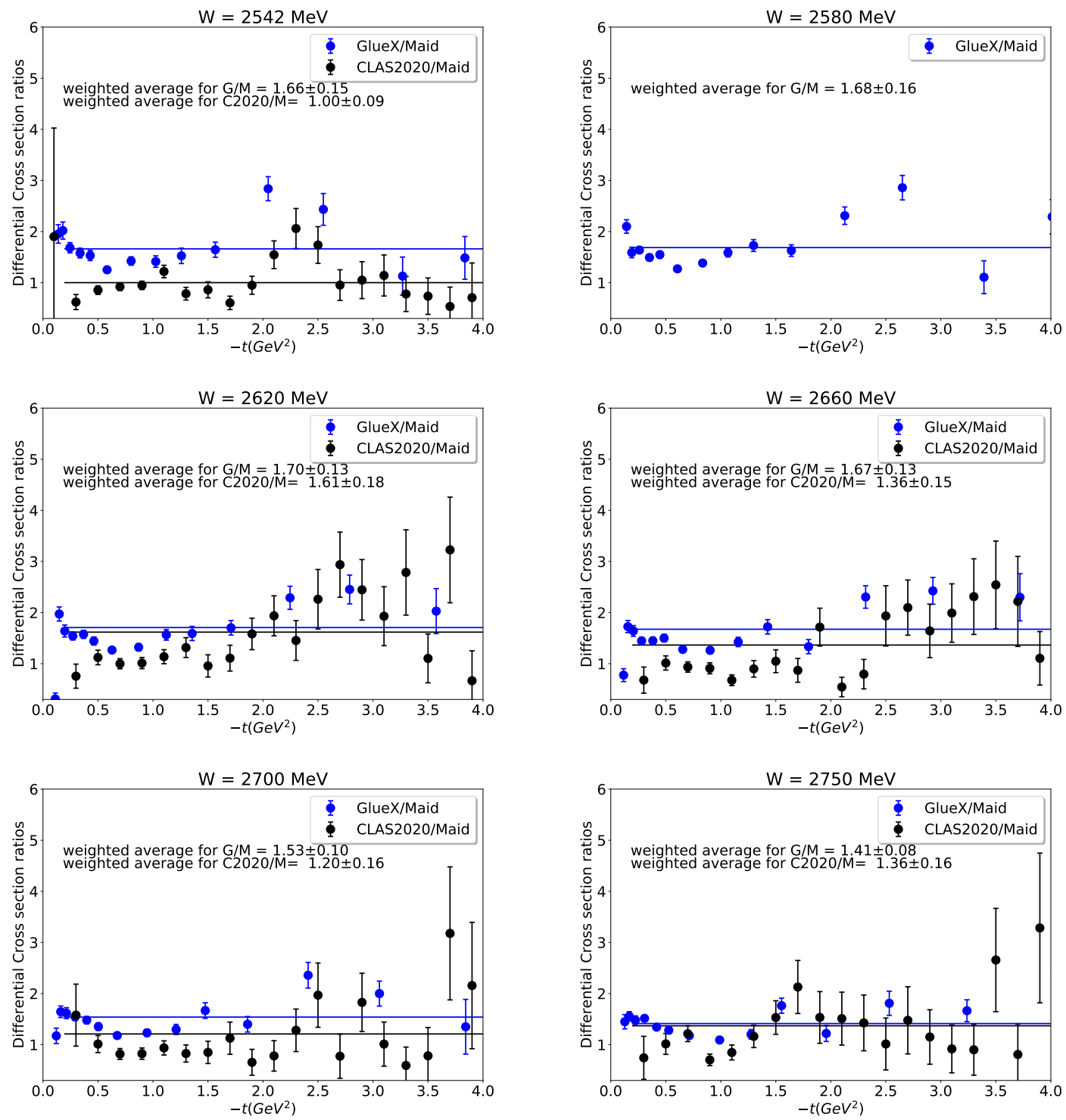

Figure 4.7: Weighted average differential cross section ratios between GlueX and EtaMAID2018 (blue), and CLAS2020 and EtaMAID2018 (black), as a function of -t. W = $2.542 \mathrm{GeV}$ up to $\mathrm{W}=2.750 \mathrm{GeV}$. 
Weighted Average Differential Cross Section Ratios, $\mathrm{W}=2810 \mathrm{MeV}$ up to $\mathrm{W}=3277 \mathrm{MeV}$.
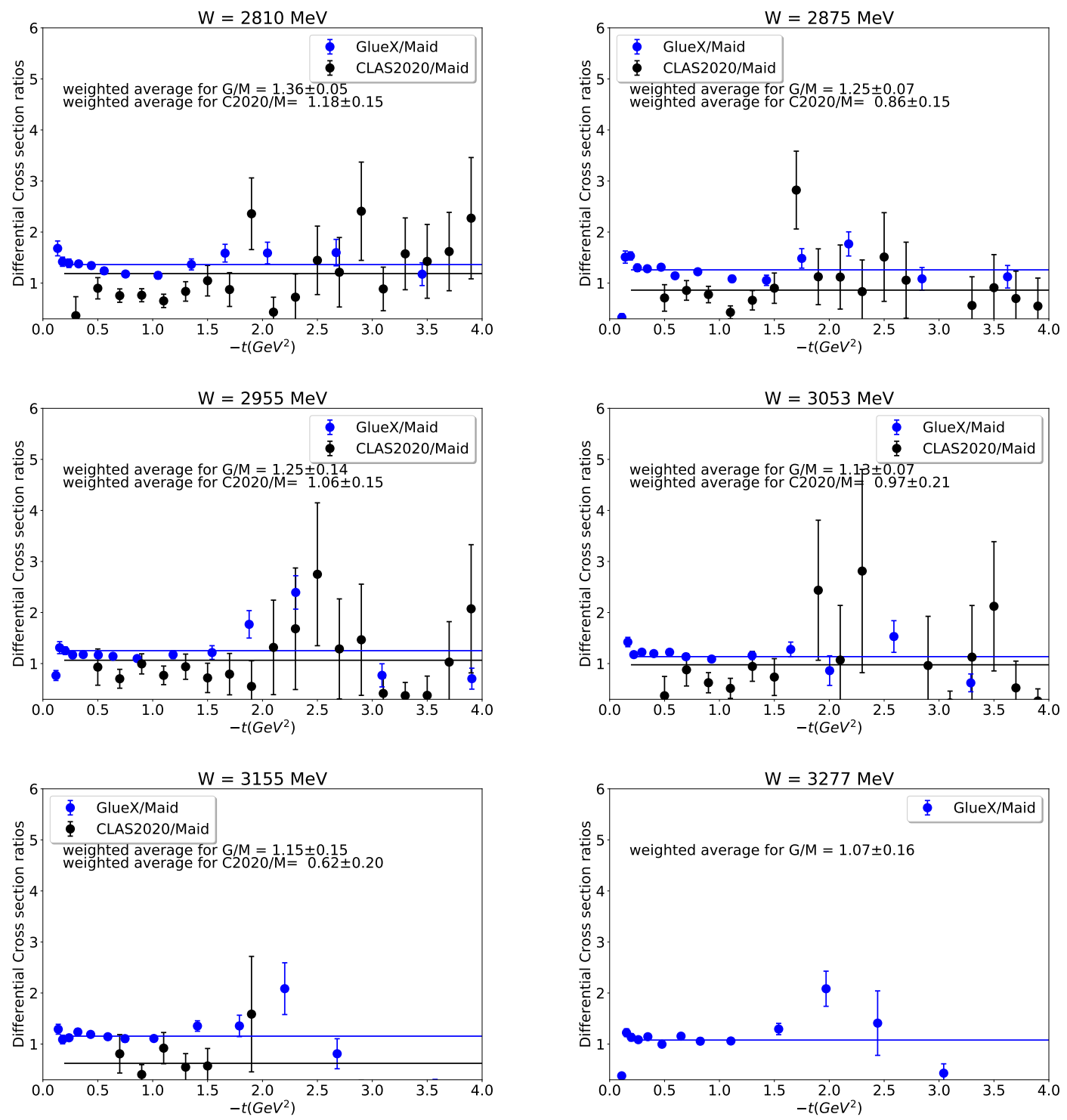

Figure 4.8: Same as 4.7, but for $\mathrm{W}=2.810 \mathrm{GeV}$ up to $\mathrm{W}=3.277 \mathrm{GeV}$. 
$\log (d \sigma / d t)$ Vs. $-\mathbf{t}, 2.54<W<2.78 \mathbf{G e V}$
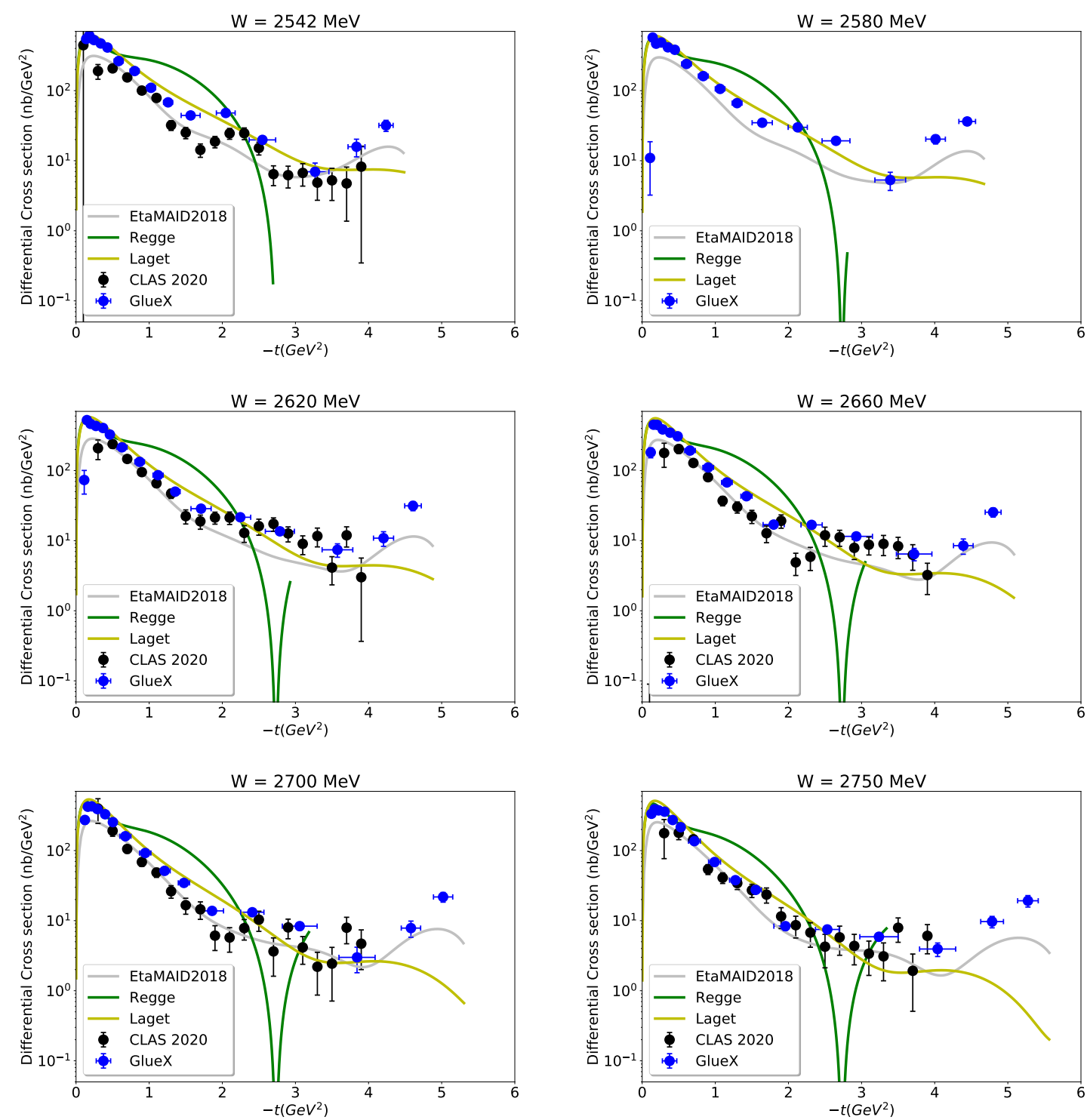

Figure 4.9: Differential cross section of $\eta$ mesons in a log-scale as a function of -t. $\mathrm{W}=$ $2.542 \mathrm{GeV}$ up to $\mathrm{W}=2.750 \mathrm{GeV}$. 
$\log (d \sigma / d t)$ Vs. $-\mathbf{t}, 2.78<W<3.36 \mathbf{G e V}$.
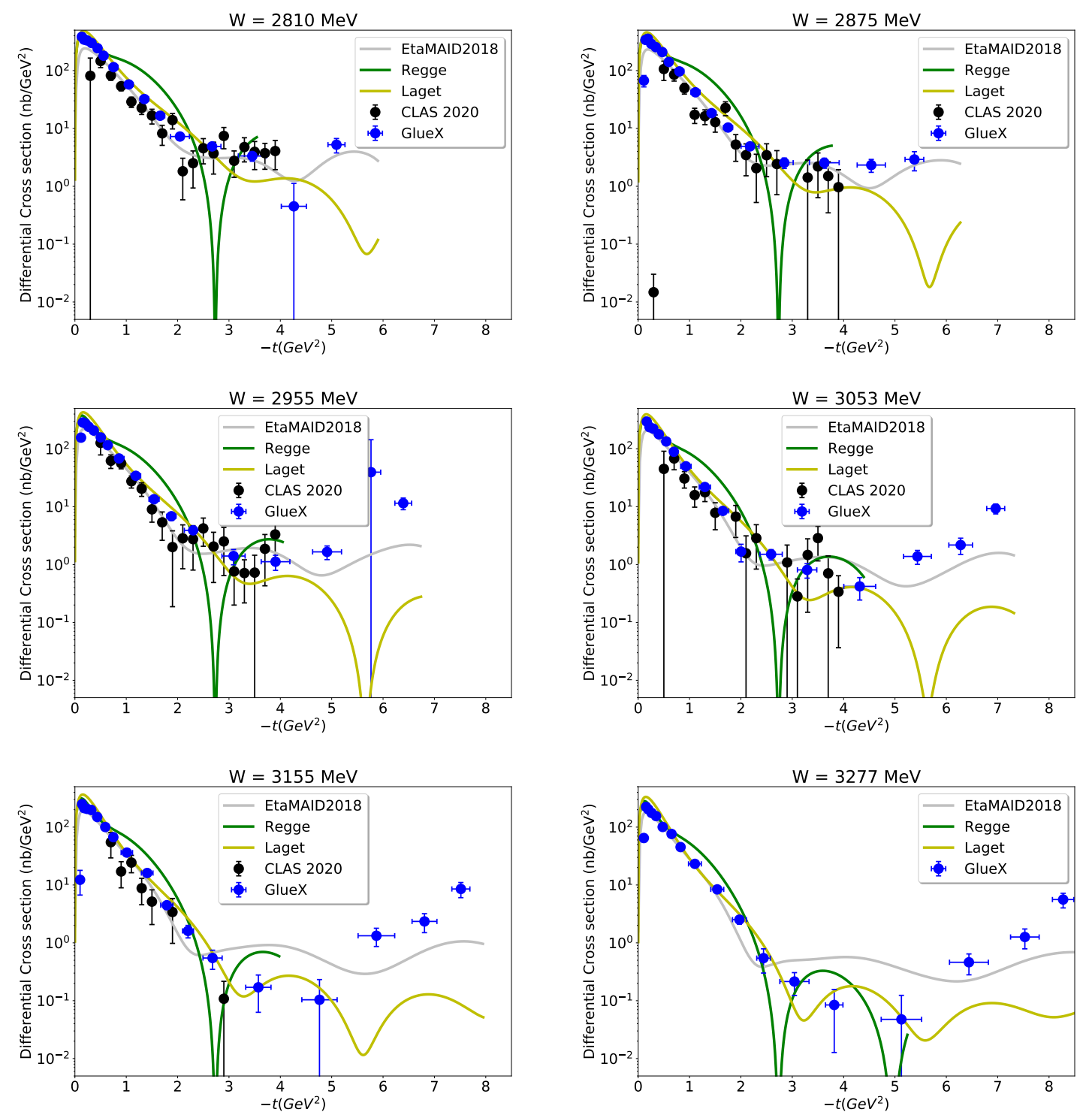

Figure 4.10: Same as Fig. 4.9, but for $\mathrm{W}=2.810 \mathrm{GeV}$ up to $\mathrm{W}=3.277 \mathrm{GeV}$. 
Differential Cross Section as a function of $-\mathrm{t}$
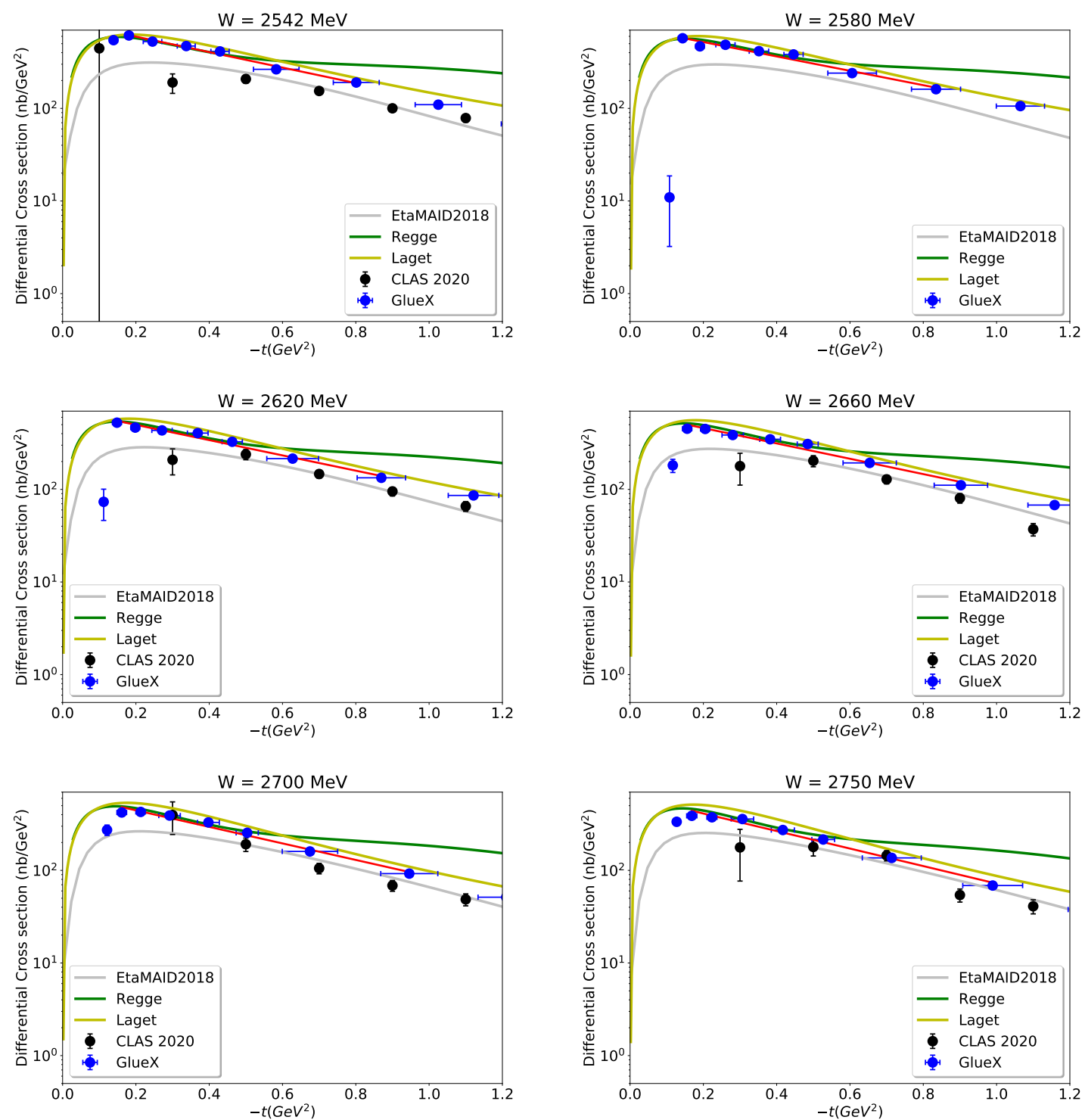

Figure 4.11: Differential cross section of $\eta$ mesons for $-\mathrm{t}<1.2 \mathrm{GeV}^{2}$ at $\mathrm{W}=2.542 \mathrm{GeV}$ up to $\mathrm{W}=2.750 \mathrm{GeV}$. The red line is the linear fit in the range of $0.14<-t<1 \mathrm{GeV}^{2}$ 
In order to see the details for large $(-\mathrm{t})>2.5 \mathrm{GeV}^{2}$, the $\log (d \sigma / d t)$ results are presented in Figs 4.9, and 4.10. The GlueX data have a wider cross section coverage (- $\mathrm{t}>4 G e V^{2}$ ) than CLAS. There is a minimum in $d \sigma / d t$ at around $-t \backsim 3 G e V^{2}$, after which the production mechanism is dominated by baryon exchange ( $\mathrm{u}$-channel processes). The location of these (-t) minima increase with increasing $\mathrm{W}$. The corresponding $\mathrm{u}$-value of these minima can be calculated using the equations in section 1.4. These minima are located at $u \sim 1 G e V^{2}$. There are two different slopes in agreement with the Regge trajectories in the forward (low - $t$, high $\mathrm{u}$ ) and backward direction (high - $\mathrm{t}$, low $\mathrm{u}$ ). The EtaMAID model cross section predictions agree with the data for $\mathrm{W}>3.053 \mathrm{GeV}^{2}$ in a relatively wide t-range of $0.3<-t<2.0$. There were no previous data available for large (-t) with high statistics, and high energies.

Figs 4.11 , and 4.12 show $d \sigma / d t$ over a small range of $-t<1.2 G e V^{2}$. The red line is a linear fit for the points in a small t-range $\left(0.14<-t<1 G e V^{2}\right)$. The extrapolation of this line to $\mathrm{t}=0$ gives the value of $C_{0}$ of equation 1.36. Although $\mathrm{t}=0$ is nonphysical, the $t_{\min }$ derived in chapter 1 for the used center of mass energies is very small and is very close to zero. The value of $d \sigma / d t$ at $\mathrm{t}=t_{\min }$ is proportional to the total cross section $\sigma_{\text {total }}$. These results will be discussed further in chapter 5 , with similar results from the high energy data. 
Differential Cross Section as a function of -t.
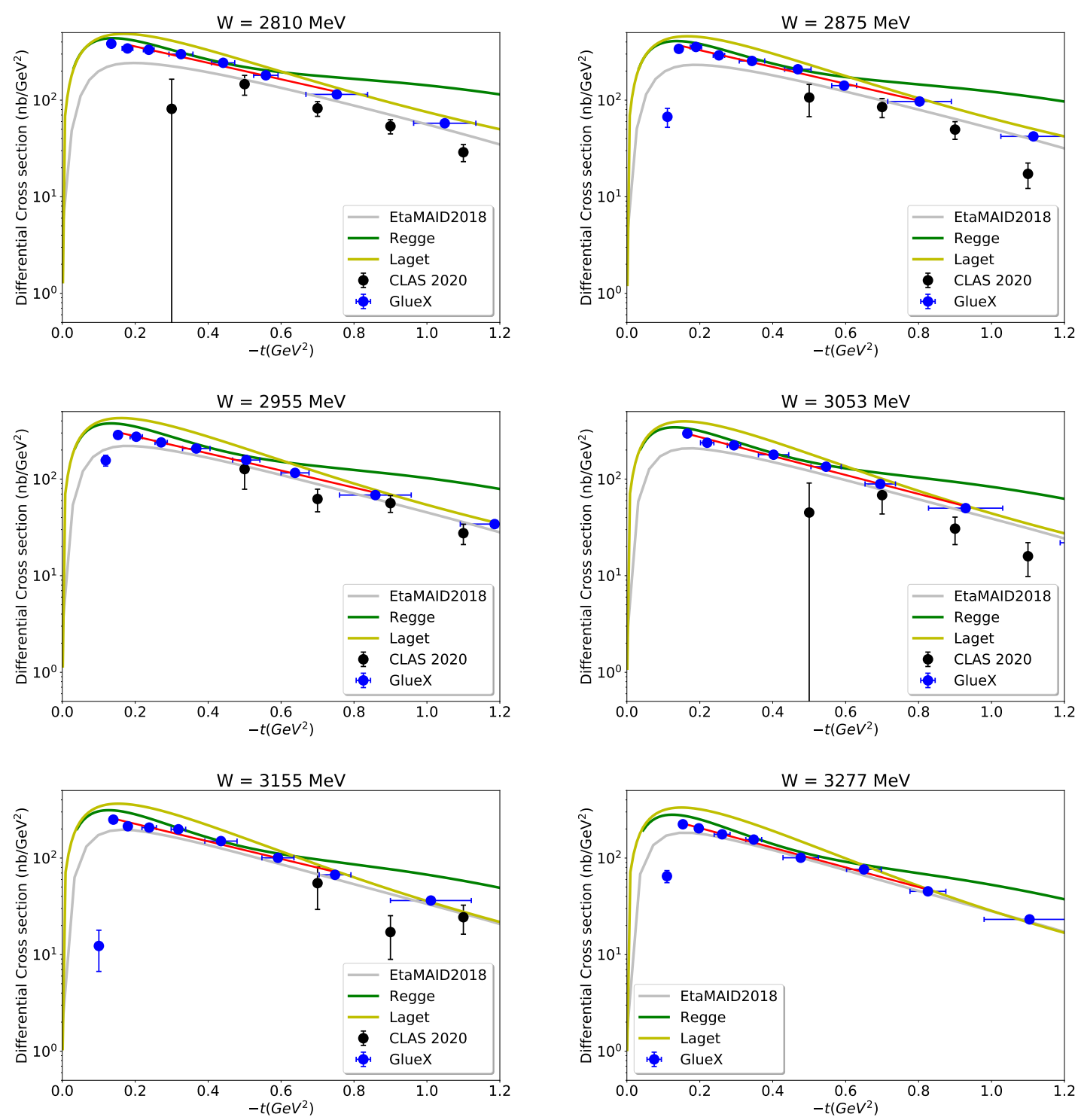

Figure 4.12: Same as Fig. 4.11, but for $\mathrm{W}=2.810 \mathrm{GeV}$ up to $\mathrm{W}=3.277 \mathrm{GeV}$. 


\subsection{Systematic Studies}

In this section the sensitivity of the experimental differential cross sections $(d \sigma / d \Omega)$ will be studied. A simulated Monte-Carlo data set was generated using the phase space generator(genr8), combined with the genEtaRegge sample in order to have more statistics in the backward direction, then the acceptance and hence, the cross sections were calculated. In order to quantify the cross section variation as a function of the variation of an analysis parameter, the ratio $N_{\sigma_{i}}$ is formed as:

$$
N_{\sigma_{i}}=\frac{\sigma_{i}-\sigma_{n}}{\Delta \sigma_{n}}
$$

Where $\sigma_{i}$ refers to the differential cross section with a certain variation of an analysis parameter, and $\sigma_{n}\left(\Delta \sigma_{n}\right)$ is the nominal differential cross section (and its uncertainty). Figs 4.13, and 4.14 show $N_{\sigma_{i}}$ when the acceptance is calculated using simulated data from the combined genr8 and genEtaRegge Monte-Carlo samples. The difference in the cross section is less than half of the nominal error bars for most of the points.

$N_{\sigma_{i}}$ has also been determined for each nominal cut that has been applied during the event selection. The kinematic fitting $\chi^{2}$ cut was varied between 20 and 40, while the missing mass squared cut was changed between $0.02 \mathrm{GeV}^{2}$ and $0.04 \mathrm{GeV}^{2}$. The vertex z-cut is also changed to be $52.0<z<78.0 \mathrm{~cm}$, where the target length would be $L_{T}=26$ $\mathrm{cm}$. Another wider cut, where $50.0<z<80.0 \mathrm{~cm}$, with full target length of $30 \mathrm{~cm}$ is used. The recoil proton momentum cut has been varied between $300 \mathrm{MeV}$ and $400 \mathrm{MeV}$.

The mean values of $N_{\sigma_{i}}$ in most of the energy bins are smaller than 0.5. This indicates that the systematic error due to an individual cut variation is less than half of the error calculated in section 4.1. The standard deviations $(\sigma)$ for $N_{\sigma_{i}}$ have been calculated for each cut at each center of mass energy bin. The standard deviations due to the cut variation upper and lower limits are written on each bin, see as an example Figs 4.15, and 4.16 . The differential cross section variations due to the $\chi^{2}$ cut is in general the largest variation compared to all the other ones. $N_{\sigma_{i}}$ for the $M_{M}^{2}$ (z-vertex cut) cut is shown in Figs. 4.17, 


\section{Normalized Differential Cross Section with genr8 events added.}
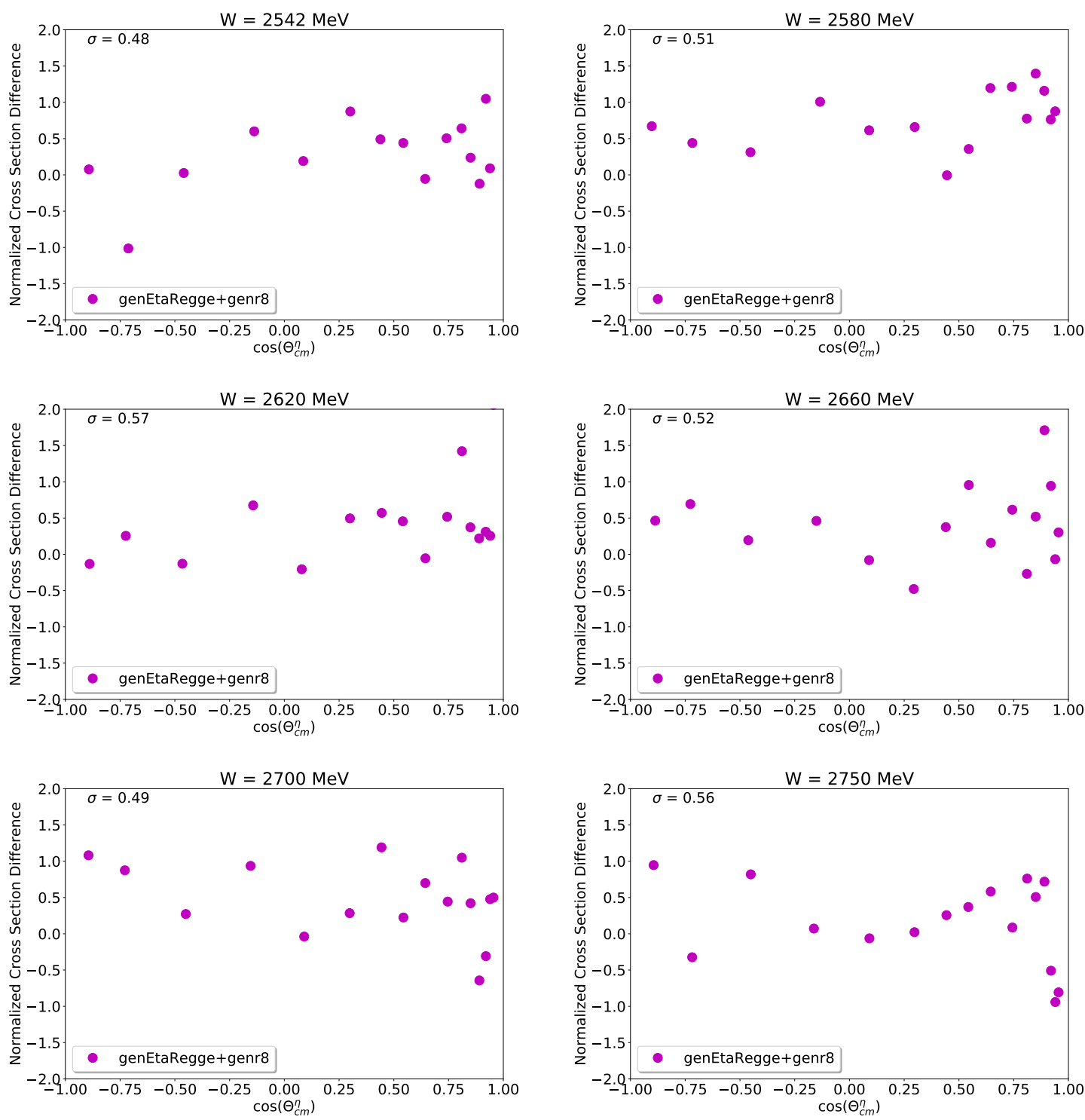

Figure 4.13: Normalized differential cross section difference using genr8 events in addition to genEtaRegge. 


\section{Normalized Differential Cross Section with genr8 events added.}
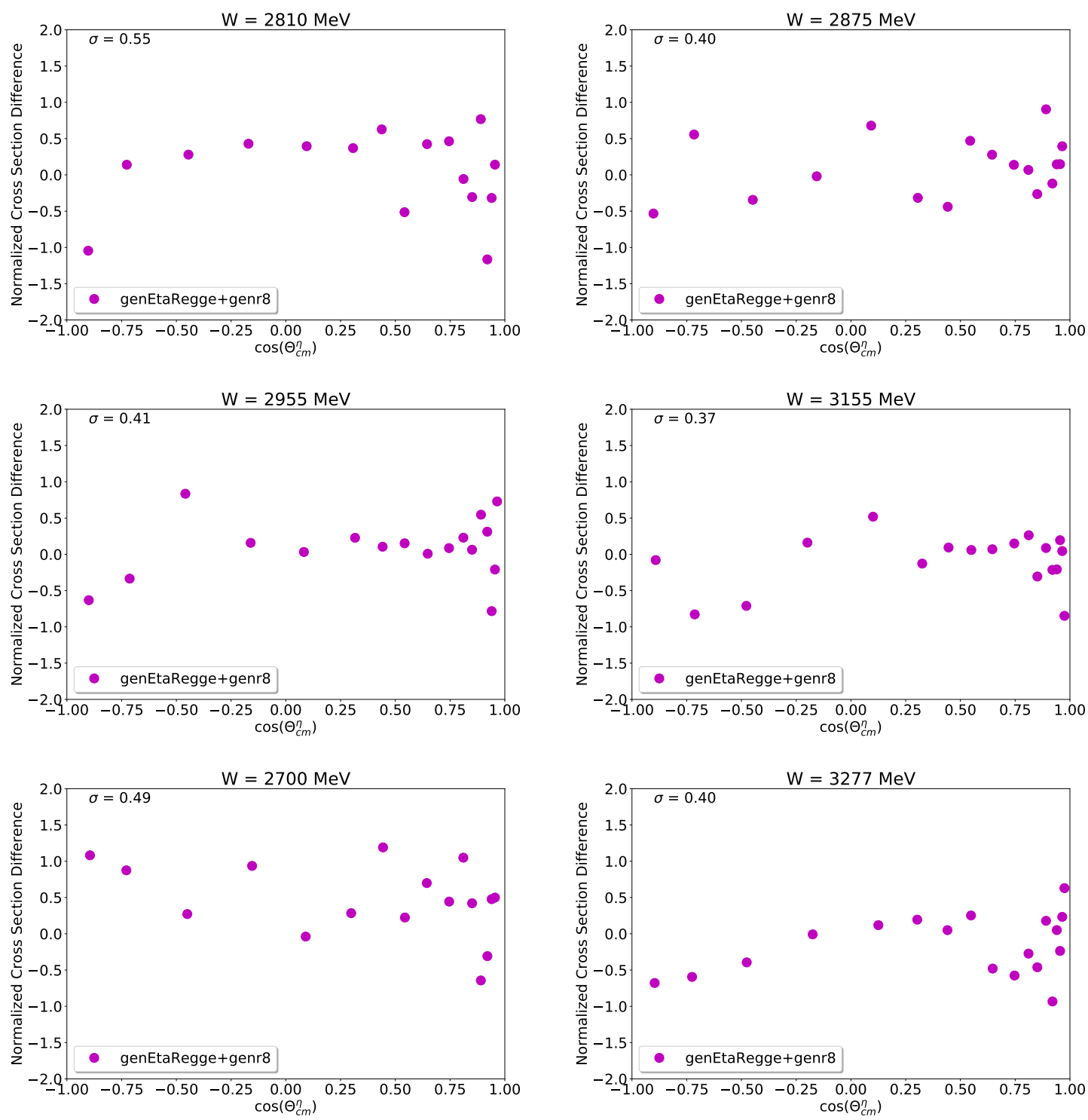

Figure 4.14: Normalized differential cross section difference using genr8 events in addition to genEtaRegge for $\mathrm{W}=2810 \mathrm{MeV}$ up to $3277 \mathrm{MeV}$. 
and 4.18 (Figs. 4.19, and 4.20). The $N_{\sigma_{i}}$ for the proton momentum cuts are shown in Fig. 4.21, and 4.22. Clearly this cut affects only the data for $\cos \theta_{c m}^{\eta}>0.92$.

In order to calculate a conservative systematic error for a cut variation that has upper (u), and lower (l) cut limits, the mean of $N_{\sigma_{i}}$ and its standard deviation $(\sigma(u), \sigma(l))$ is calculated for upper and lower cut limits, respectively. A conservative systematic error for each energy bin as a result of a parameter variation (i), is then given by $\sigma_{s y s_{i}}=\max (\sigma(u), \sigma(l))$. An overall conservative systematic error estimate $\left(s y s_{e r r}\right)$ is then calculated for each $\mathrm{W}$ bin as follows:

$$
\text { sys }_{\text {err }}=\sqrt{\sum_{i} \sigma_{s y s_{i}}^{2}}
$$

Where i runs over all analysis parameters. This conservative systematic error is then calculated for each $\left(\cos \theta_{c m}^{\eta}\right)$ in that center of mass energy $\mathrm{W}$, and tabulated in appendix A. 


\section{Normalized Differential Cross Section for $\chi^{2}$ Cuts.}
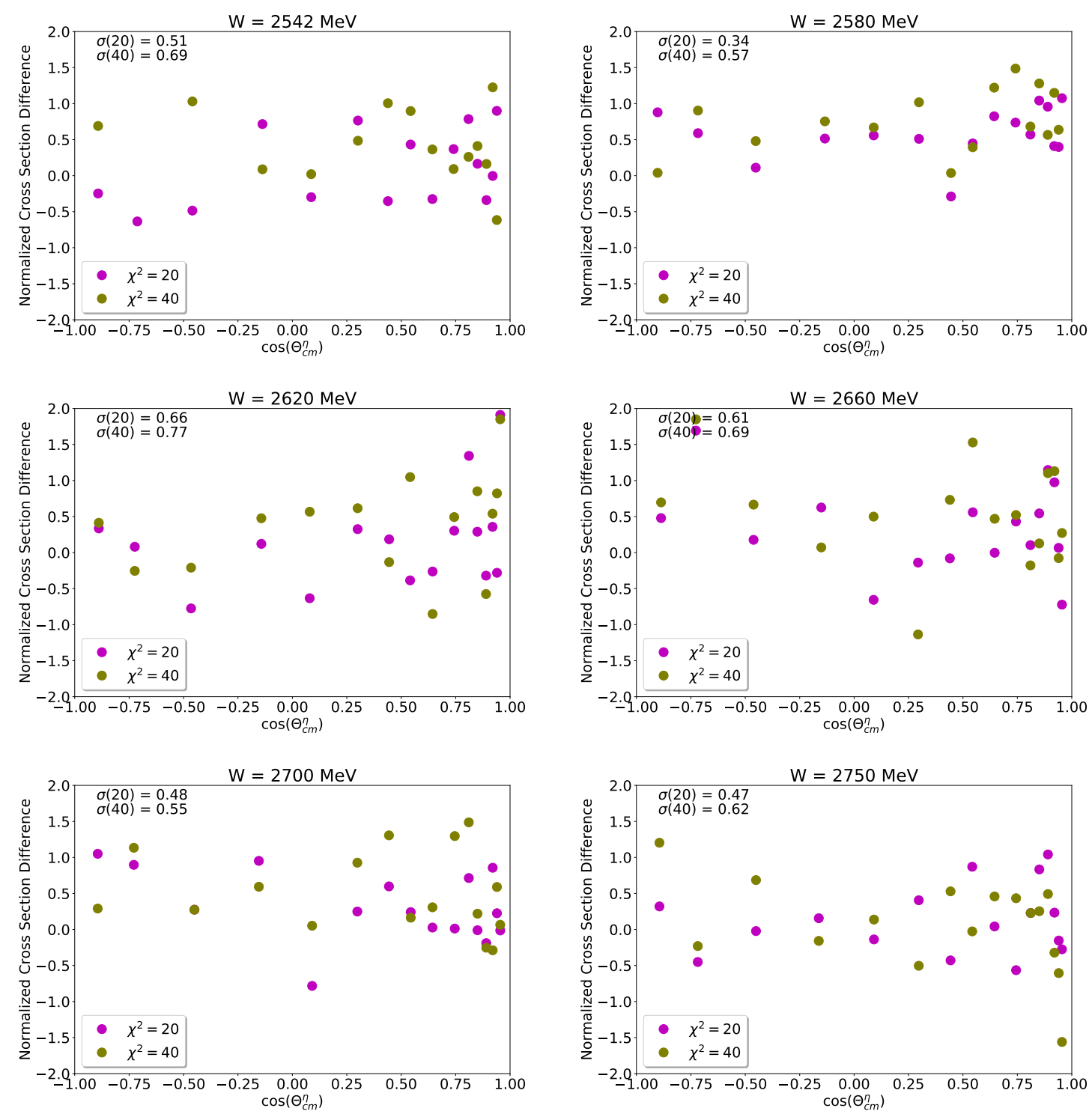

Figure 4.15: Normalized differential cross section difference for $\chi^{2}$ cuts. Magenta points are the cut of $\chi^{2}<20$, while the green points are $\chi^{2}<40$. 
Normalized Differential Cross Section for $\chi^{2}$ Cuts.
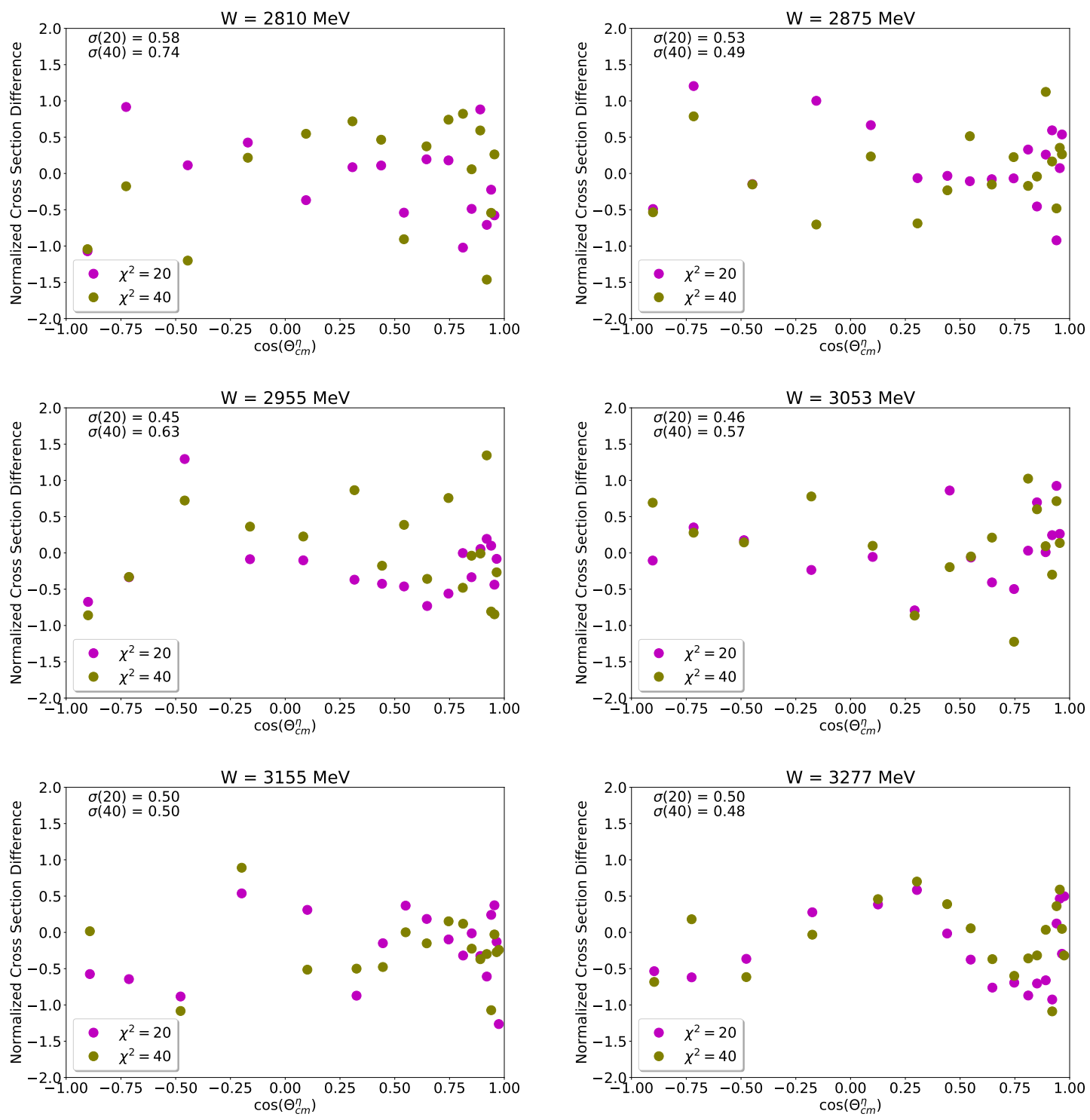

Figure 4.16: Same as 4.15 , but $\mathrm{W}=2.810 \mathrm{GeV}$ up to $\mathrm{W}=3.277 \mathrm{GeV}$. 
Normalized Differential Cross Section for Missing Mass Squared Cuts.
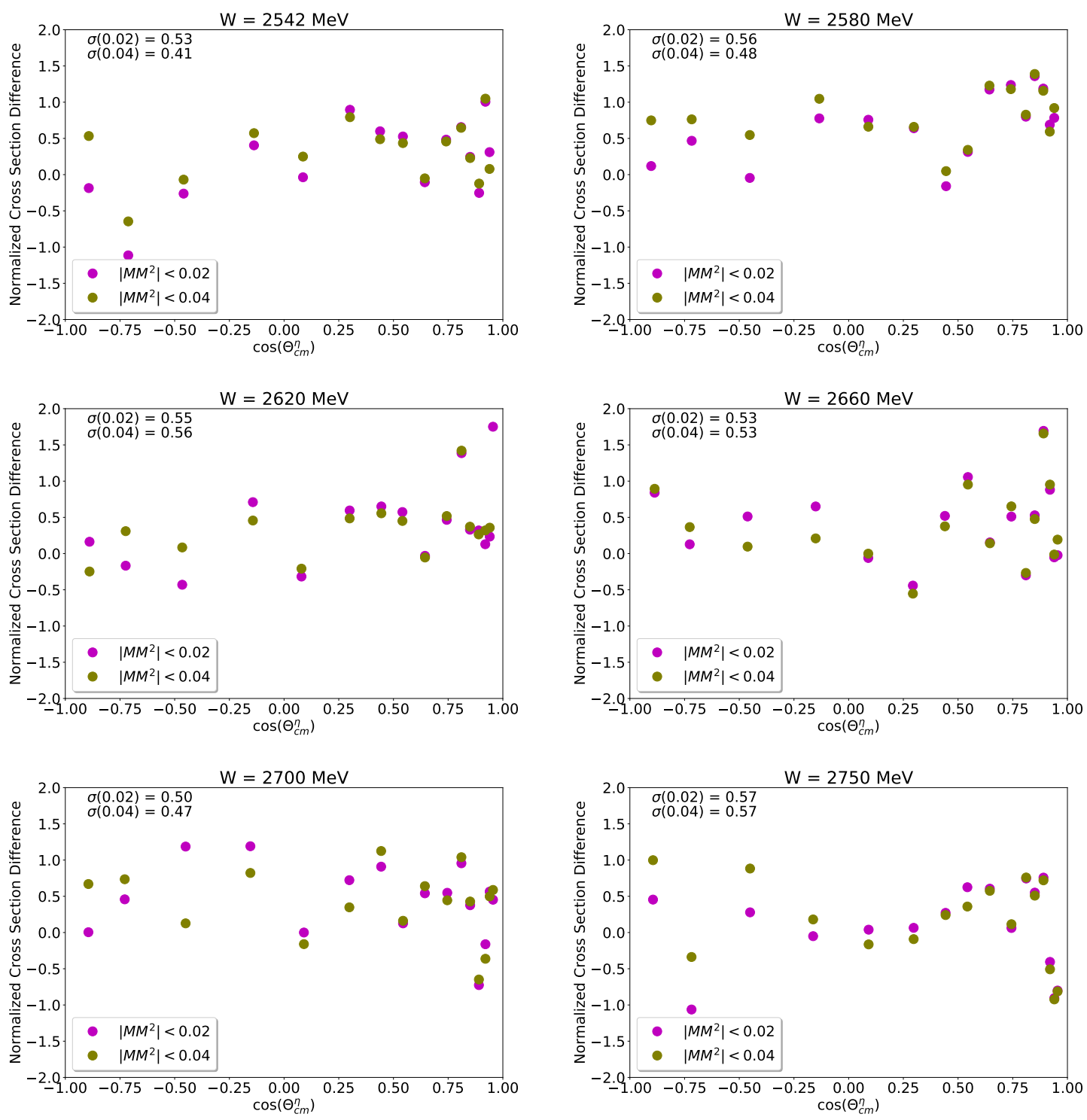

Figure 4.17: Normalized differential cross section differences for missing mass squared cuts. $\left|M_{M}^{2}\right|<0.020 \mathrm{GeV}^{2}$ in magenta, and $\left|M_{M}^{2}\right|<0.040 \mathrm{GeV}^{2}$ in green. 
Normalized Differential Cross Section for Missing Mass Squared Cuts.
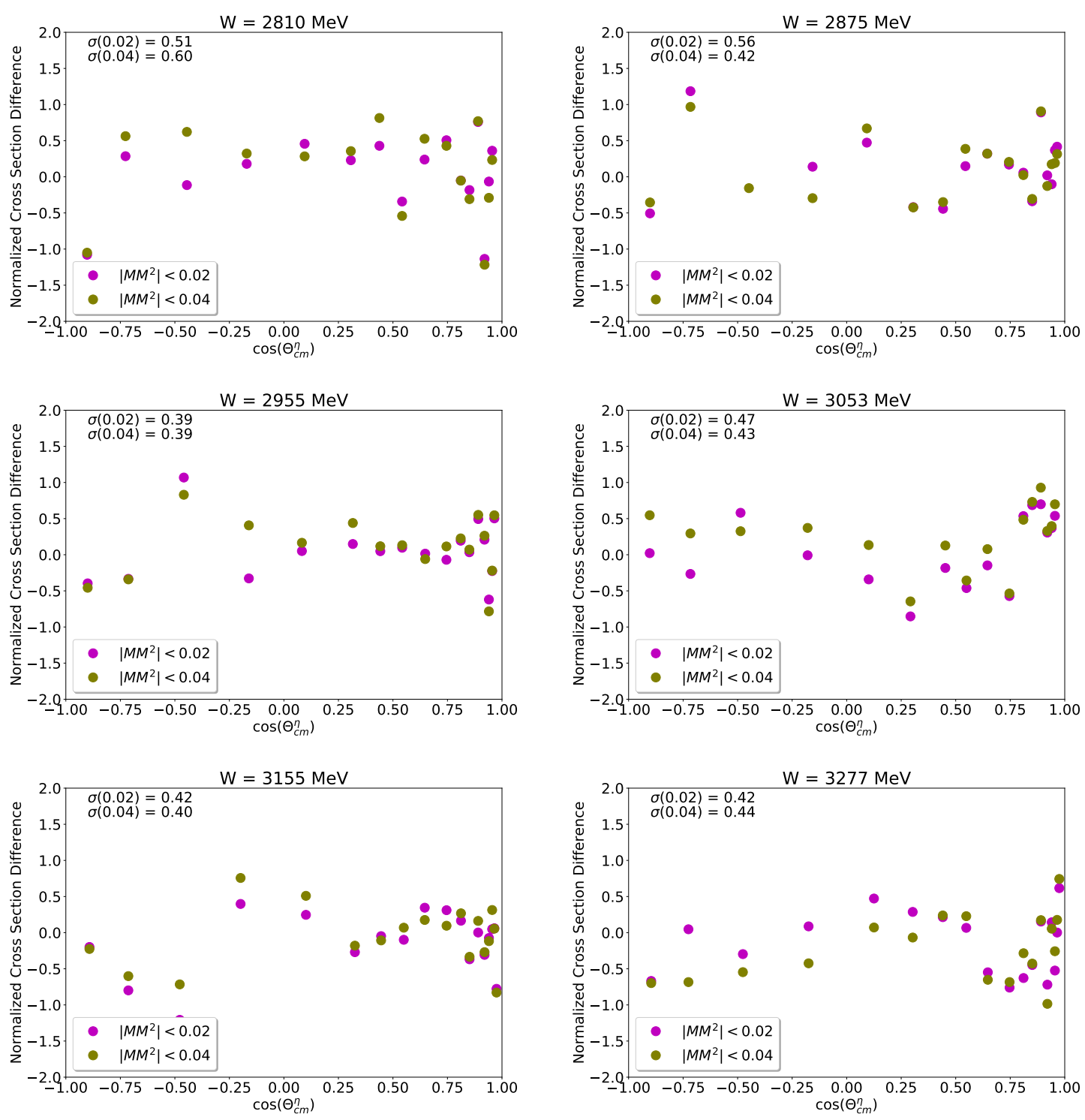

Figure 4.18: Same as 4.17 , but $\mathrm{W}=2.810 \mathrm{GeV}$ up to $\mathrm{W}=3.277 \mathrm{GeV}$. 


\section{Normalized Differential Cross Section for z-vertex Cuts.}
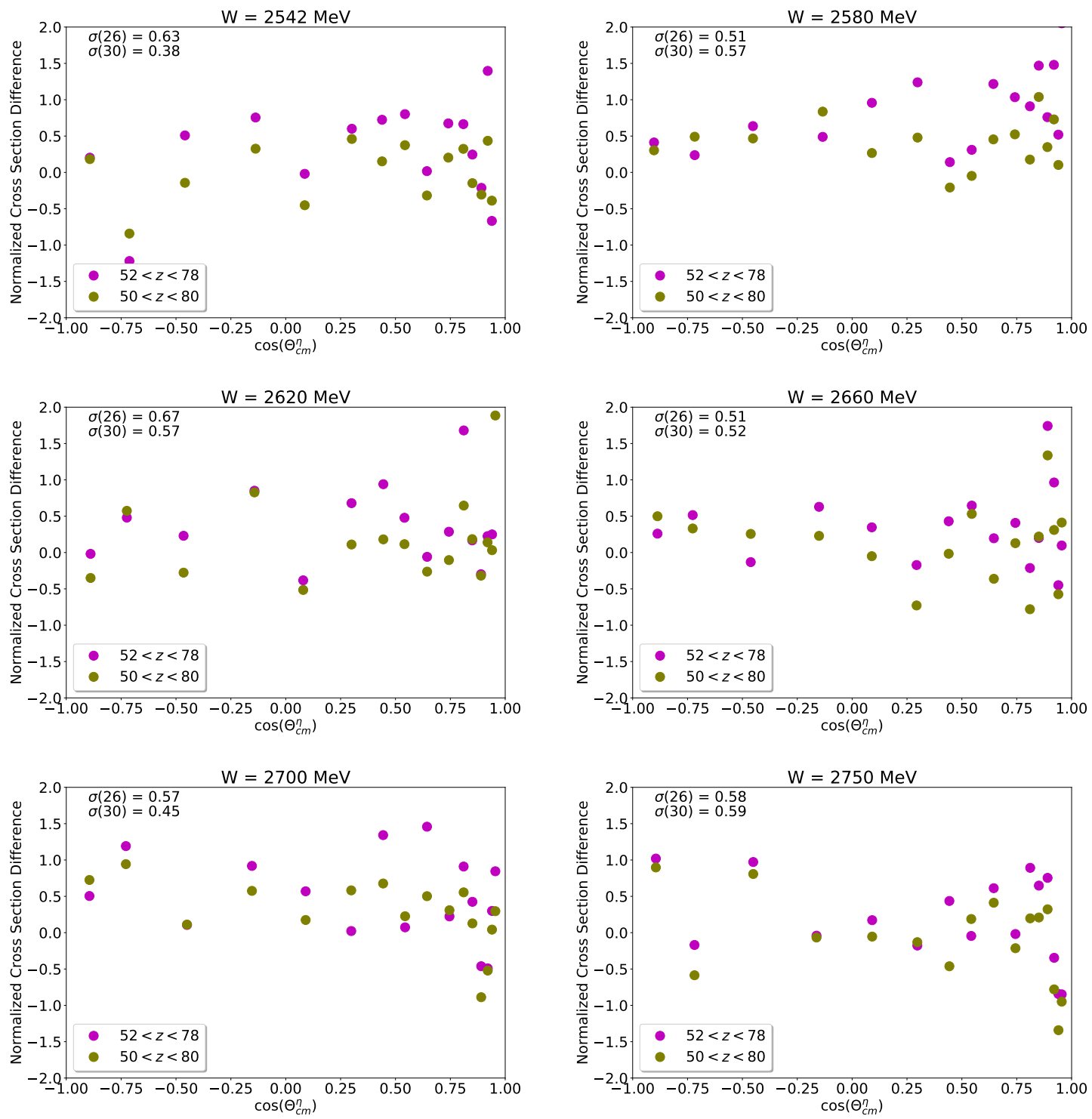

Figure 4.19: Normalized differential cross section differences for z-vertex cuts for $52<z<$ $78 \mathrm{~cm}$ in magenta, and for $50<z<80 \mathrm{~cm}$ in green. 


\section{Normalized Differential Cross Section for $\chi^{2}$ Cuts.}
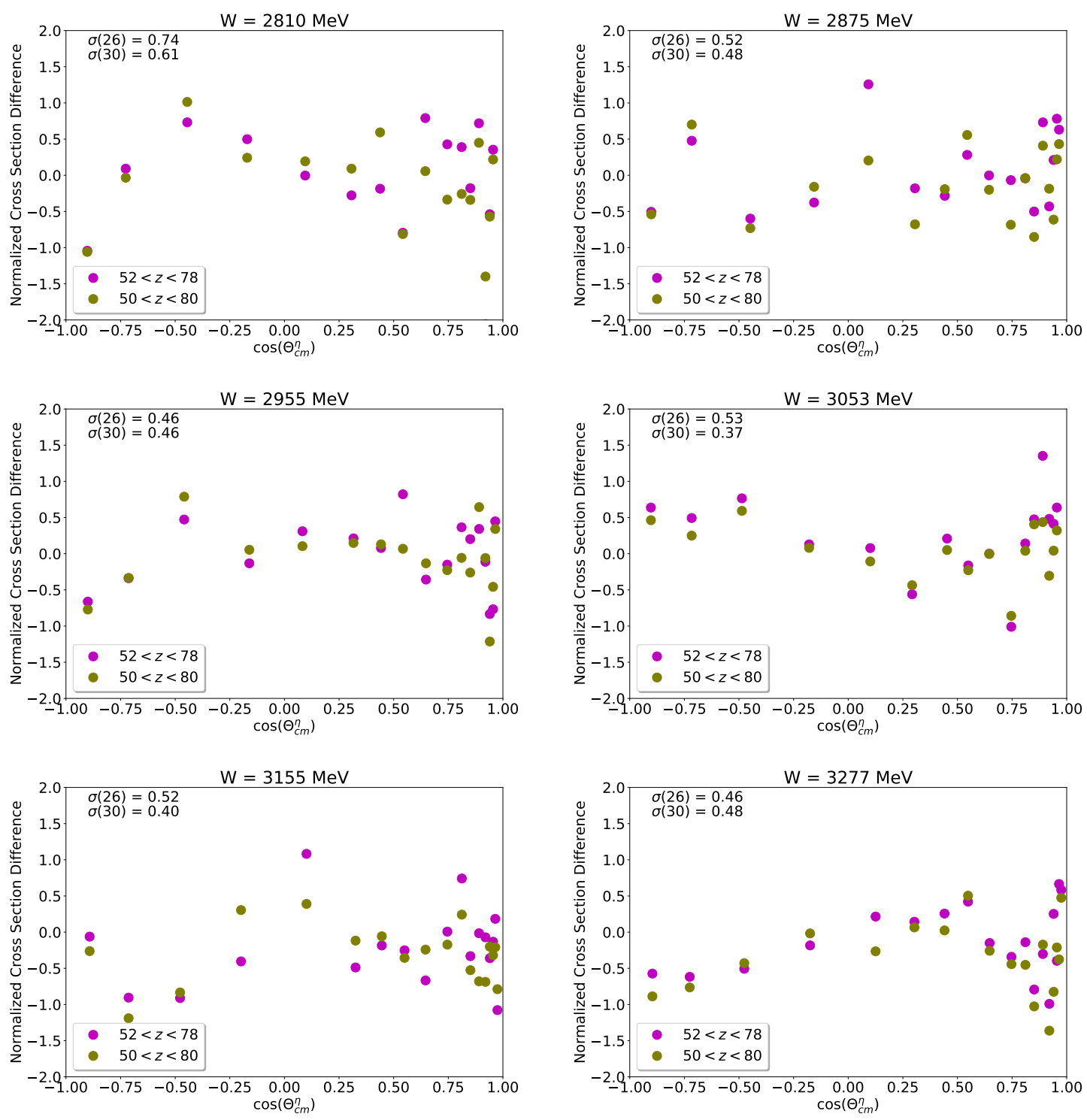

Figure 4.20: Same as 4.19 , but $\mathrm{W}=2.810 \mathrm{GeV}$ up to $\mathrm{W}=3.277 \mathrm{GeV}$. 


\section{Normalized Differential Cross Section for Proton momentum Cuts.}
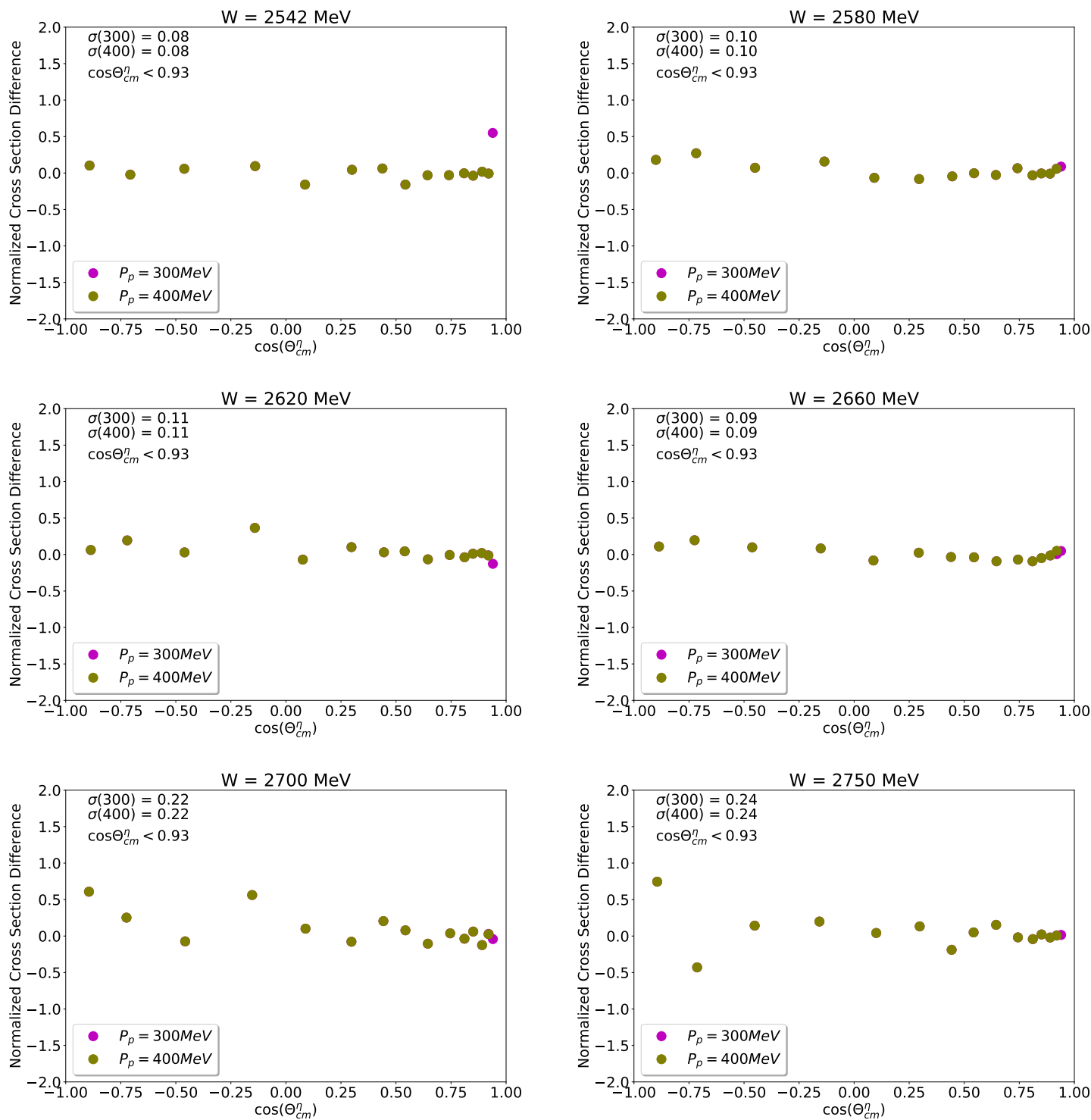

Figure 4.21: Normalized differential cross section differences for z-vertex cuts for proton momentum $<400 \mathrm{MeV}$ in green and for proton momentum $<300 \mathrm{MeV}$ in magenta. 


\section{Normalized Differential Cross Section for Proton momentum Cuts.}
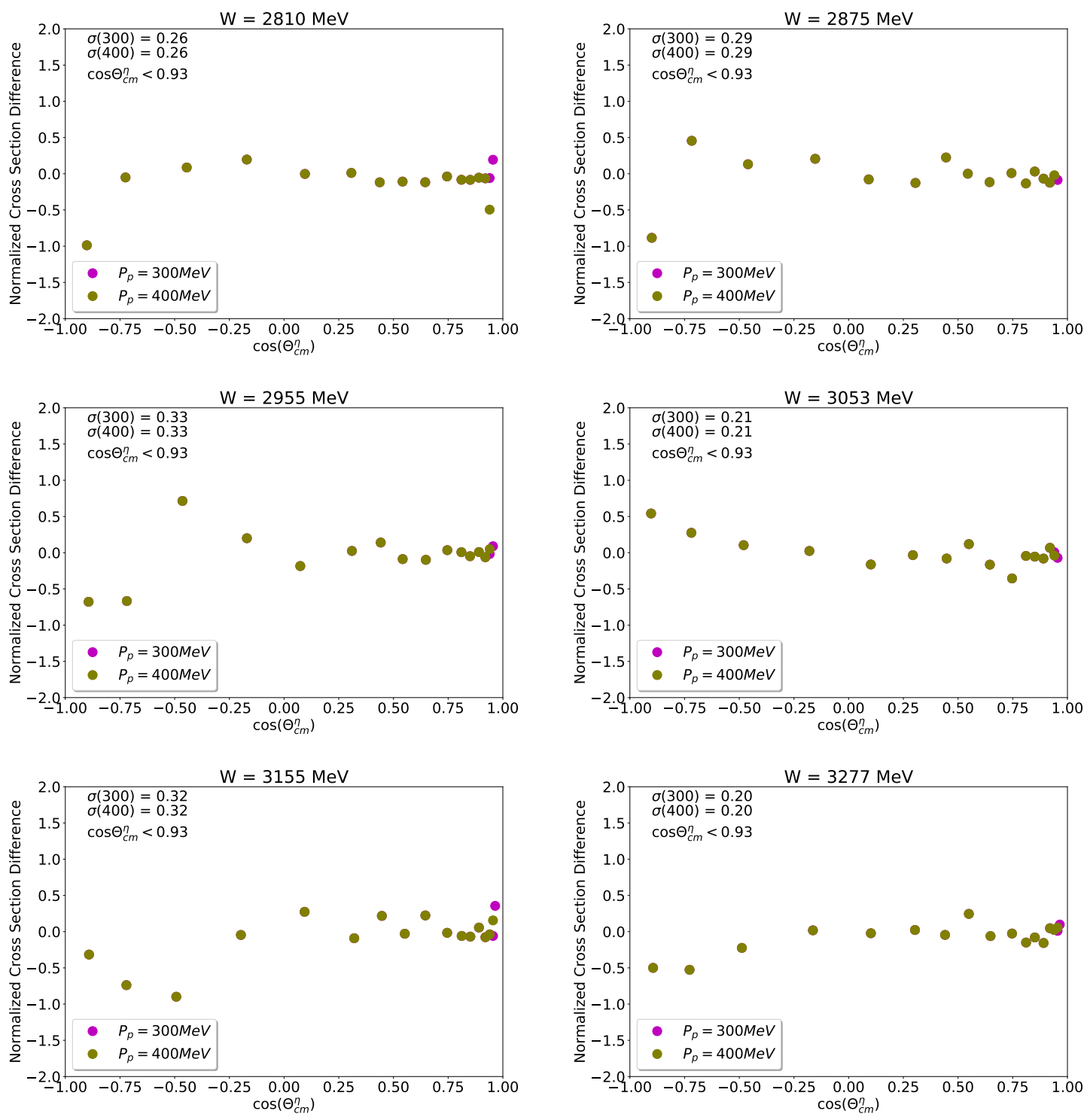

Figure 4.22: Same as 4.19 , but $\mathrm{W}=2.810 \mathrm{GeV}$ up to $\mathrm{W}=3.277 \mathrm{GeV}$. 


\section{CHAPTER 5}

\section{Differential Cross Sections For $\eta$-Photo-production at High Beam Energies}

\section{$6.2 \mathrm{GeV} \leq E_{\gamma} \leq 11.6 \mathrm{GeV}$}

$\eta$-Photo-production cross sections have also been determined at higher photon energies between $6.2 \mathrm{GeV}$ and $11.6 \mathrm{GeV}$ corresponding to center of mass energies $3.54 \mathrm{GeV}<W<$ 4.8 GeV. These cross sections have been determined from the Gluex Spring 2017 data set. The beam energy range has been divided into 12 bins of nearly equal width of about 450 $\mathrm{MeV}$. The center of each beam energy bin and the corresponding central value of the center of mass energy bins are shown in table 5.1. The photon flux has been measured by the pair spectrometer as discussed in chapter 2. Fig. 5.1 shows the photon flux distribution as a function of the central $\mathrm{W}$ values. The coherent peak includes three $\mathrm{W}$ bins, $W=3.93,4.04$, and $4.14 \mathrm{GeV}$.

The event selection for the 2017 high energy data set is the same as for the the low energy data and was discussed in details in the previous chapter. The missing mass squared cut of $\left|M_{\text {missing }}^{2}\right|<0.03 \mathrm{GeV}^{2}$ is shown in Fig. 5.2. The final $\eta$ mass distribution is shown in Fig. 5.3. This data set contains considerably more events than the low energy data set as we recorded a total of $472,873 \eta$ events in this data set compared to 58,488 in the low energy data set.

\subsection{Data and Monte-Carlo (MC) Comparisons}

The Data and Monte-Carlo comparison has been performed in a similar way as for the low energy data, see section 3.2. A sample of $10 \mathrm{M} \eta$ events in a beam energy range between 6.2 and $11.6 \mathrm{GeV}$ have been generated using the genEtaRegge generator, and the response of the GlueX detector has again been simulated using GEANT4. The comparisons between data and MC for the $\eta$ momentum and polar angle distributions are shown in Figs. 5.4, and 5.5 , respectively. There is a good agreement between data and MC especially at the high momentum end of each bin (Fig. 5.5). The low momentum peaks in the data correspond to the u-channel $\eta$ photo-production. The model does not include the u-channel production 


\begin{tabular}{|c|c|}
\hline W center value $(\mathrm{GeV})$ & E center value $(\mathrm{GeV})$ \\
\hline 3.60 & 6.5 \\
3.71 & 6.9 \\
3.82 & 7.3 \\
3.93 & 7.8 \\
4.04 & 8.2 \\
4.14 & 8.7 \\
4.24 & 9.1 \\
4.34 & 9.6 \\
4.44 & 10.0 \\
4.53 & 10.5 \\
4.62 & 10.9 \\
4.71 & 11.3 \\
\hline
\end{tabular}

Table 5.1: The central values of each center of mass energy bins and the corresponding beam energy for the high energy data.

Photon Flux from 2017 data set.

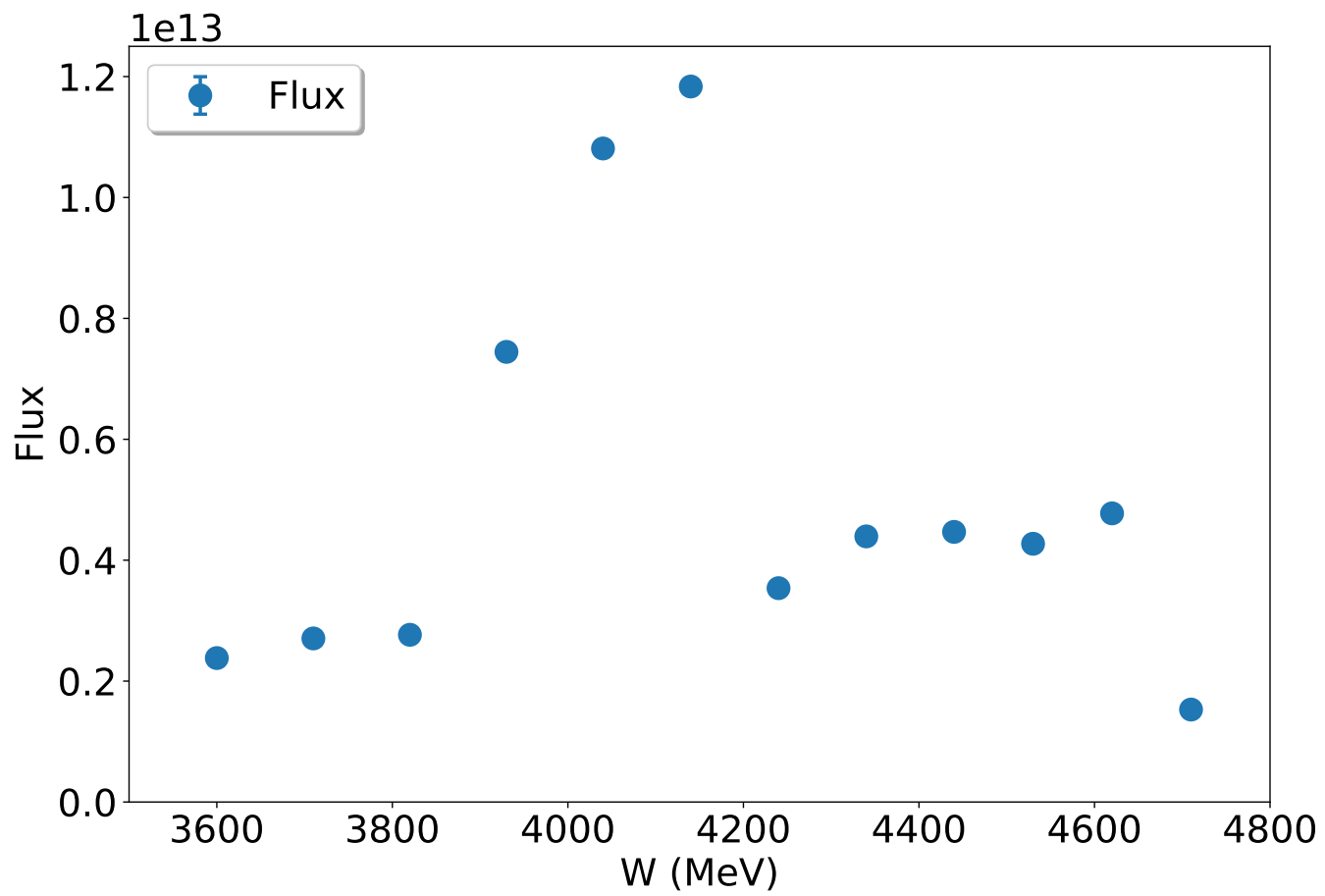

Figure 5.1: Photon flux in 2017 data set 
Missing mass squared for $\gamma p \rightarrow \eta p$ using 2017 data set.

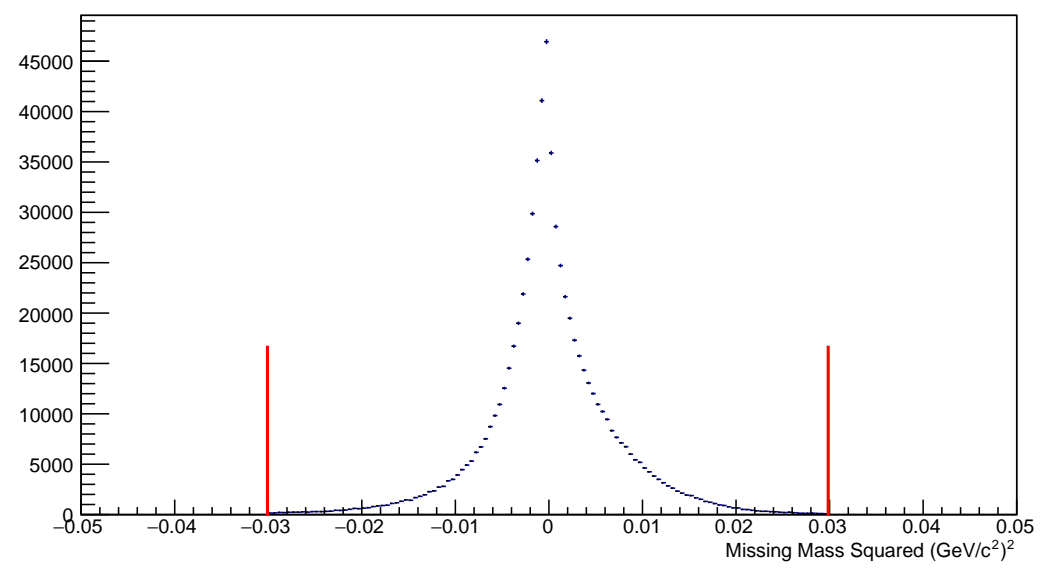

Figure 5.2: $\left|M_{\text {missing }}^{2}\right|$ distribution with cut (red line)

Mass distribution of $\eta$ mesons.

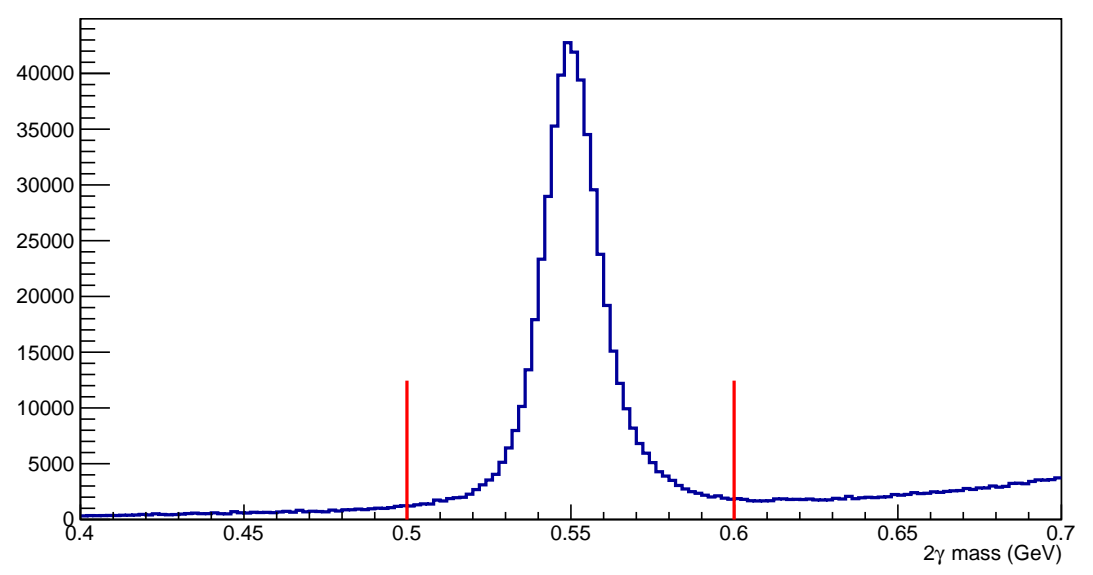

Figure 5.3: $\gamma \gamma$ mass distribution after applying all the event selection cuts. The red lines denote the mass interval over which the fit has been performed to extract the yield 
mechanism. Fig.5.4 confirms the small $\eta$ photo-production polar angle distribution in the center of mass system. As W increases the production polar angle decreases, and the t-channel forward peak magnitude increases.

\subsection{Raw Yield Extraction and Acceptance Calculations}

The yield is extracted for each $\cos \theta_{c m}^{\eta}$ in an identical way to the low energy data set, see chapter 3. The extracted yield for all $\cos \theta_{c m}^{\eta}$ in each of the center of mass energy bins is shown in Figs. 5.6, and5.7. The yield at backward angles is much smaller than at forward

angles. Therefore the inset figure is used to zoom in for $\cos \theta_{c m}^{\eta}>0.6$. The horizontal error bars are the standard deviation of $\cos \theta_{c m}^{\eta}$ in each bin. The yields are highest in $\mathrm{W}$ regions corresponding to the coherent peak because of the increased flux. At $\mathrm{W}=4.71$, the photon flux drops by an order of magnitude as shown in Fig. 5.1 and therefore the yield drops dramatically in this last energy bin.

The acceptance for the high energy data set is calculated in the same way discussed in section 3.3. The acceptance increases from a minimum of approximately $15 \%$ at $\cos \theta_{c m}^{\eta} \backsim$ -0.5 up to a maximum of approximately $50 \%$ at $\cos \theta_{c m}^{\eta} \backsim 0.5$, (see Figs 5.8, and 5.9). In the forward directions, the acceptance pattern has a second peak at very small angles, where the acceptance becomes $50 \%$. For $\mathrm{W}>4.14 \mathrm{GeV}$, the large angles have much less statistics than the forward small angles. In this work we concentrate in the t-channel $\eta$ photo-production, where most of the cross section is produced, thus we do not have large MC samples for the backward directions. 

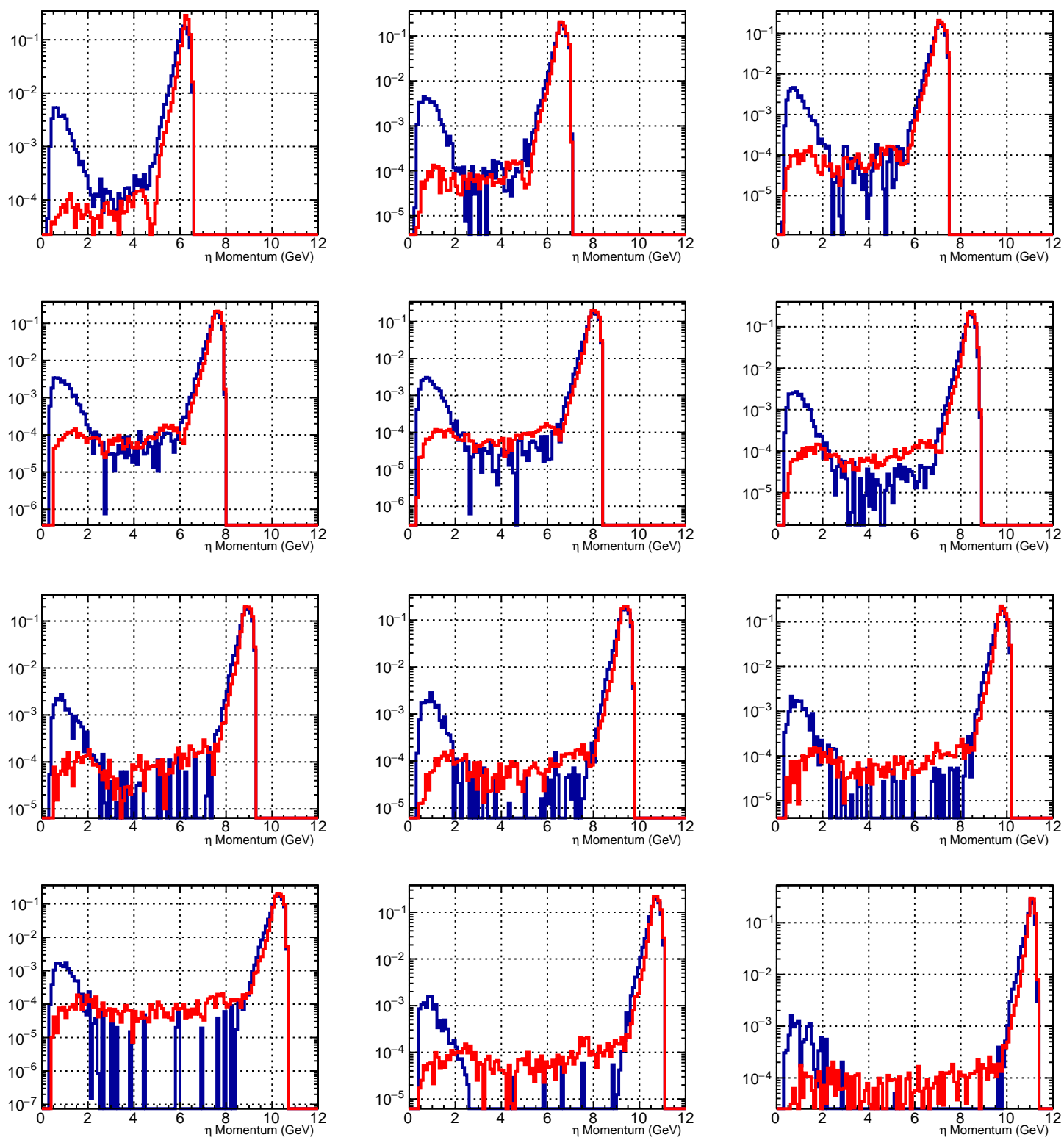

Figure 5.4: Momentum comparison of $\eta$ photo-production between data (blue) and simulation (red) in the lab frame. 

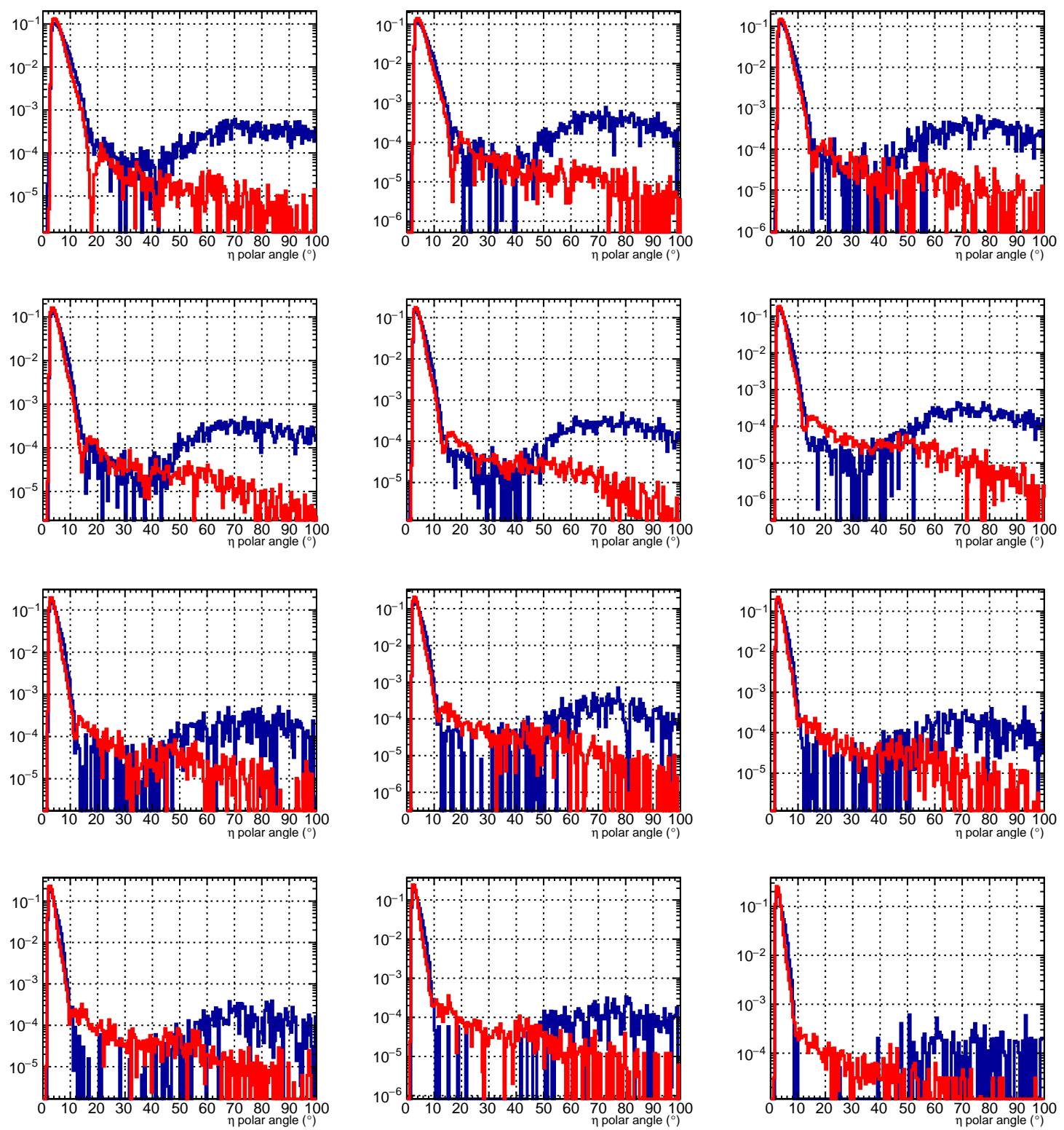

Figure 5.5: Polar angle comparison of $\eta$ photo-production between data (blue) and simulation (red) in the lab frame. 
Raw yield of $\eta$ in 2017 data set.
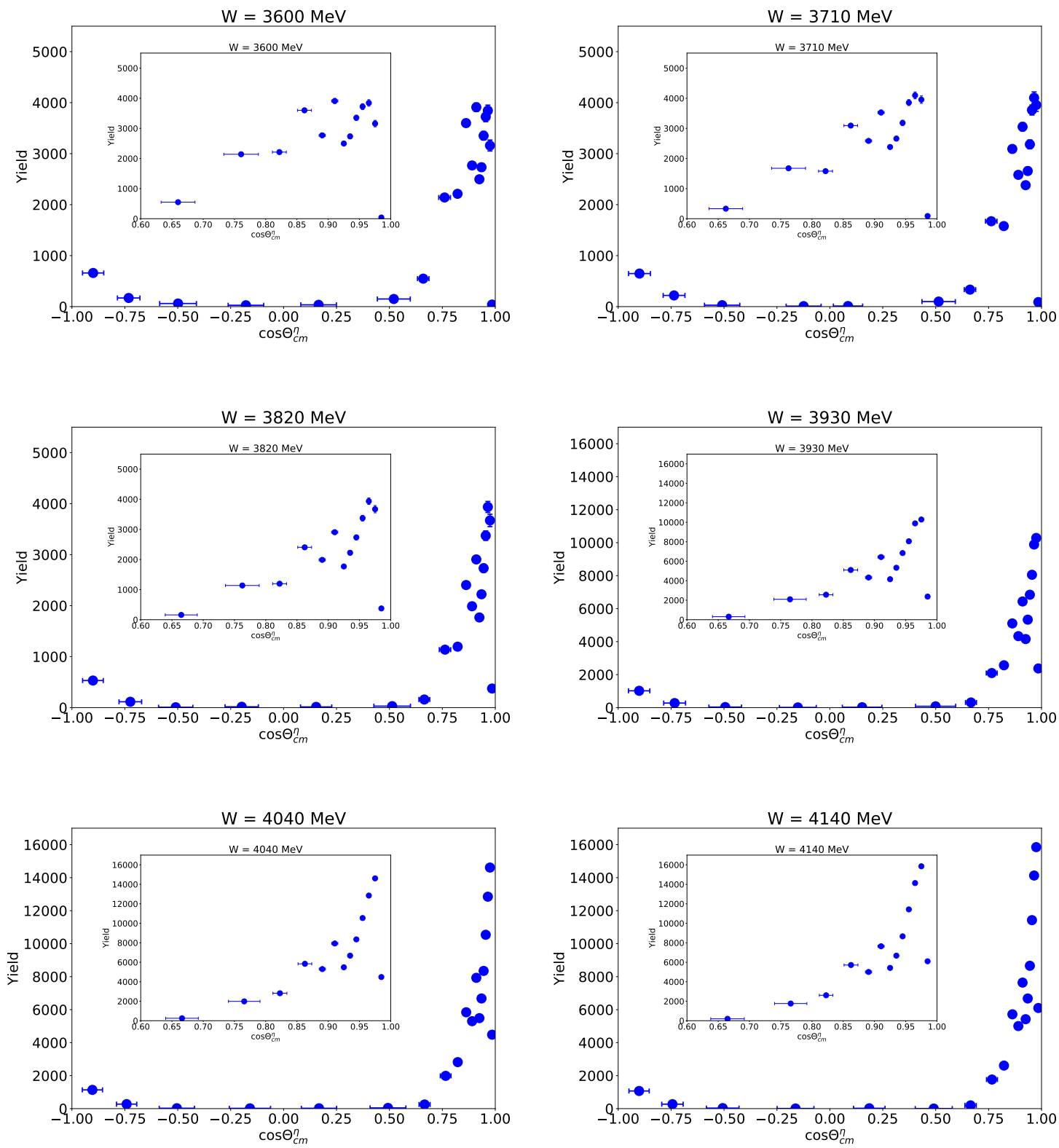

Figure 5.6: Raw $\eta 2017$ yield for 6 center of mass energy bins 3.60, 3.71, 3.82, 3.93, 4.04, and $4.14 \mathrm{GeV}$. The inset shows zoom in yield for $\cos \theta_{c m}^{\eta}>0.6$ values. 
Raw yield of $\eta$ in 2017 data set.
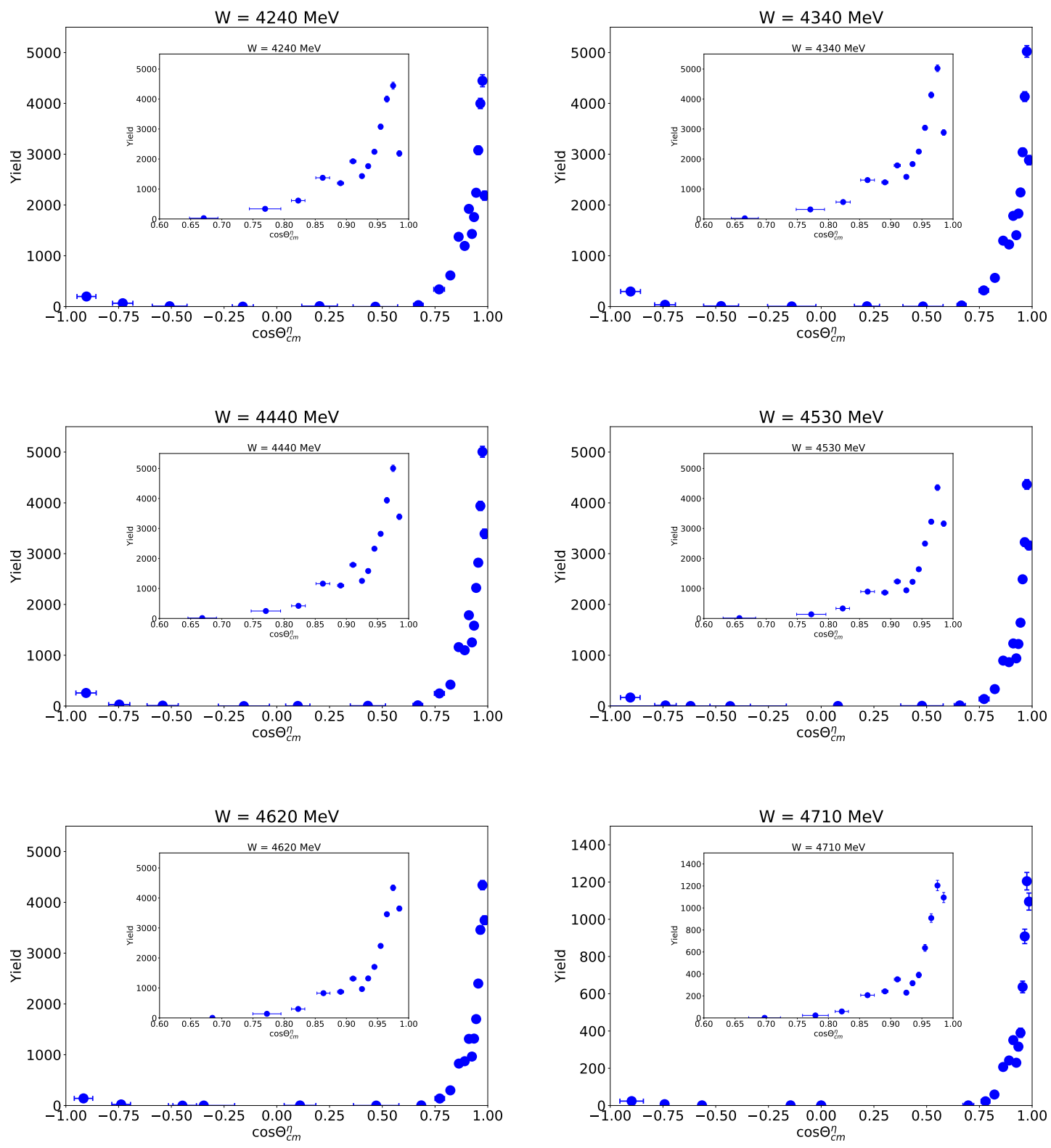

Figure 5.7: Same as Fig. 5.6 but for $\mathrm{W}$ values of 4.24, 4.34, 4.44, 4.53, 4.62 and $4.71 \mathrm{GeV}$. 
Acceptance for High Energy Data.
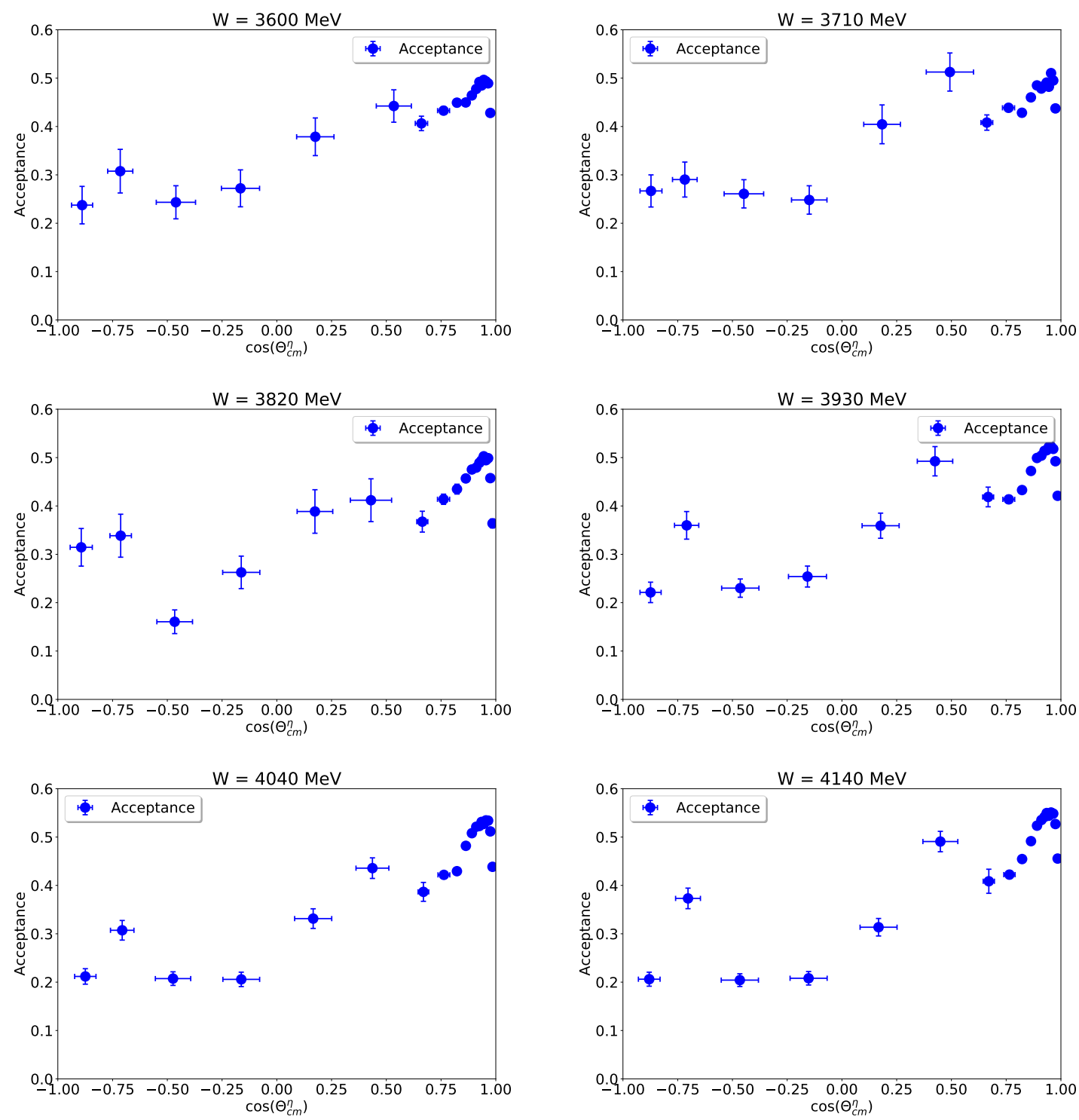

Figure 5.8: GlueX detector acceptance for 6 center of mass energy bins 3.60, 3.71, 3.82, 3.93, 4.04, and $4.14 \mathrm{GeV}$. 
Acceptance for High Energy Data.
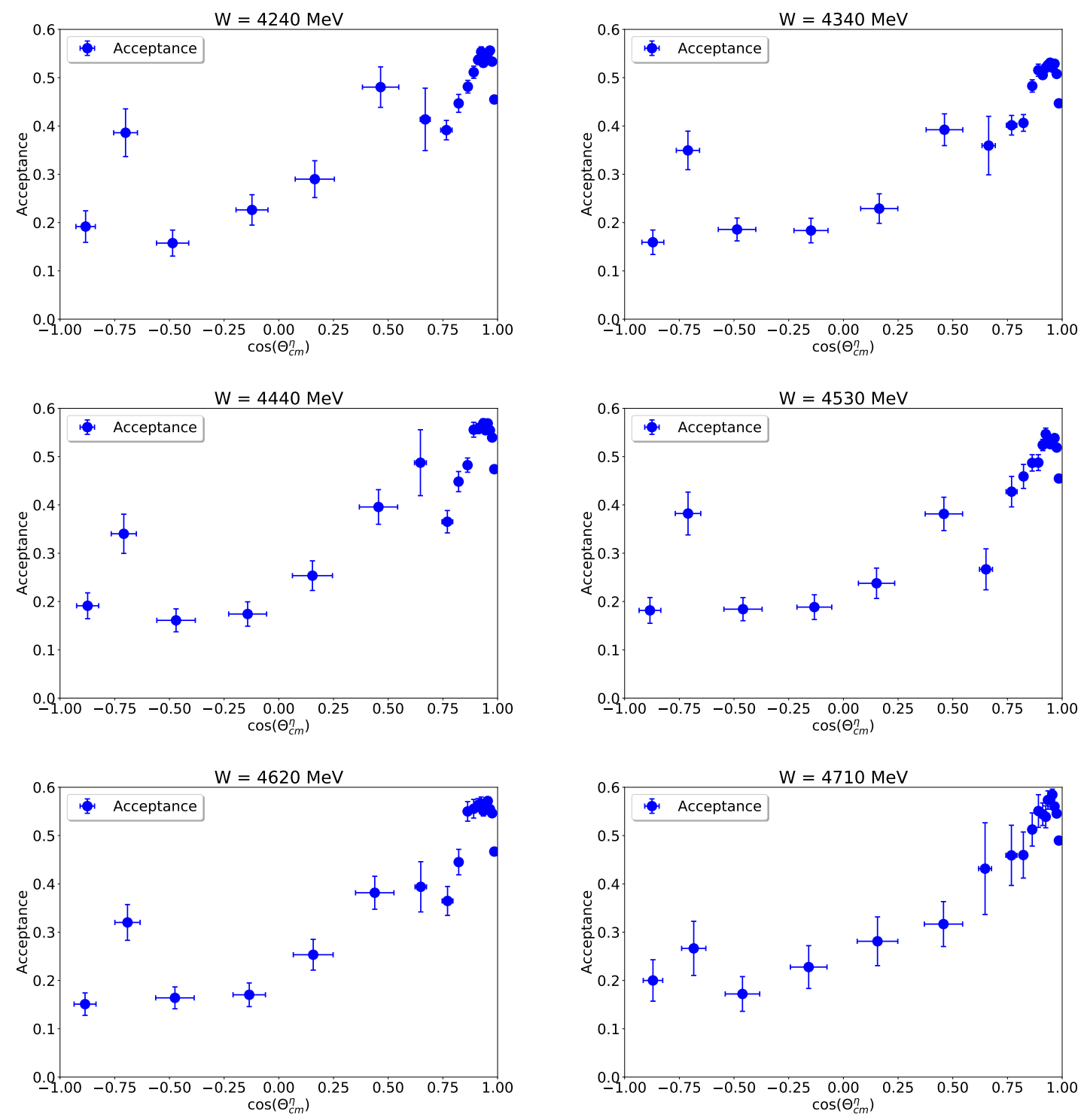

Figure 5.9: Same as 5.8, but for $W=3.60,3.71,3.82,3.93,4.04$, and $4.14 \mathrm{GeV}$. 


\subsection{Differential Cross Section Results $(d \sigma / d \Omega)$ for the High Energy Data}

The differential cross sections shown in figures 5.10, and 5.11 represent the most extensive high statistic available data set up to date for $\eta$ photo-production differential cross sections in the center of mass energies between $3.60 \mathrm{GeV}$ and $4.71 \mathrm{GeV}$. Differential cross sections have been determined for the full range of $\cos \theta_{c m}^{\eta}$, and are compared to the EtaMAID2018 and Regge model calculations. The angular distributions follow the general shape of the EtaMAID2018 model over a big range of $\cos \theta_{c m}^{\eta}$ and energy bins. For $\mathrm{W}>4140$, Fig.5.11, some angles do not have enough statistics and only an upper limit of the cross section can be provided. The forward cross section peak is larger, by at least an order of magnitude, than the backward peak at $\mathrm{W}=3.6 \mathrm{GeV}$. This difference becomes bigger for the higher energy bins.

The forward peak in the cross sections at each energy bin is shown in Fig. 5.12, and 5.13. The Regge model is overestimating the cross section for small angles by at least $50 \%$ for $\mathrm{W}<4.04 \mathrm{GeV}$. This overestimation increases with increasing $\mathrm{W}$ to reach a range of 2 to 3 times the experimental cross sections, Fig. 5.13. For $3.6 \mathrm{GeV} \leq W \leq 4.14 \mathrm{GeV}$, the EtaMaid2018 model is in good agreement within $20 \%$ of the GlueX experimental results for all polar angles with $\cos \theta_{c m}^{\eta}>0.8$, see Fig. 5.14 where the ratio of the GlueX $(\mathrm{G})$ to the EtaMAID2018(M) is calculated. Starting at $\mathrm{W}=4.24 \mathrm{GeV}$, and up to $\mathrm{W}=4.71$ $\mathrm{GeV}$, the model is overestimating the cross section for small angles where $\cos \theta_{c m}^{\eta}>0.9$. The agreement between EtaMAID2018 and the data decrease to about $70 \%$ for $\mathrm{W} \geq 4.24$ $\mathrm{GeV}$. No agreement in the forward peak is found for $\mathrm{W}>4.53$, where EtaMAID2018 over predicts the experimental data by more than $50 \%$, see Fig. 5.15 . 


\section{Differential Cross Section Results.}
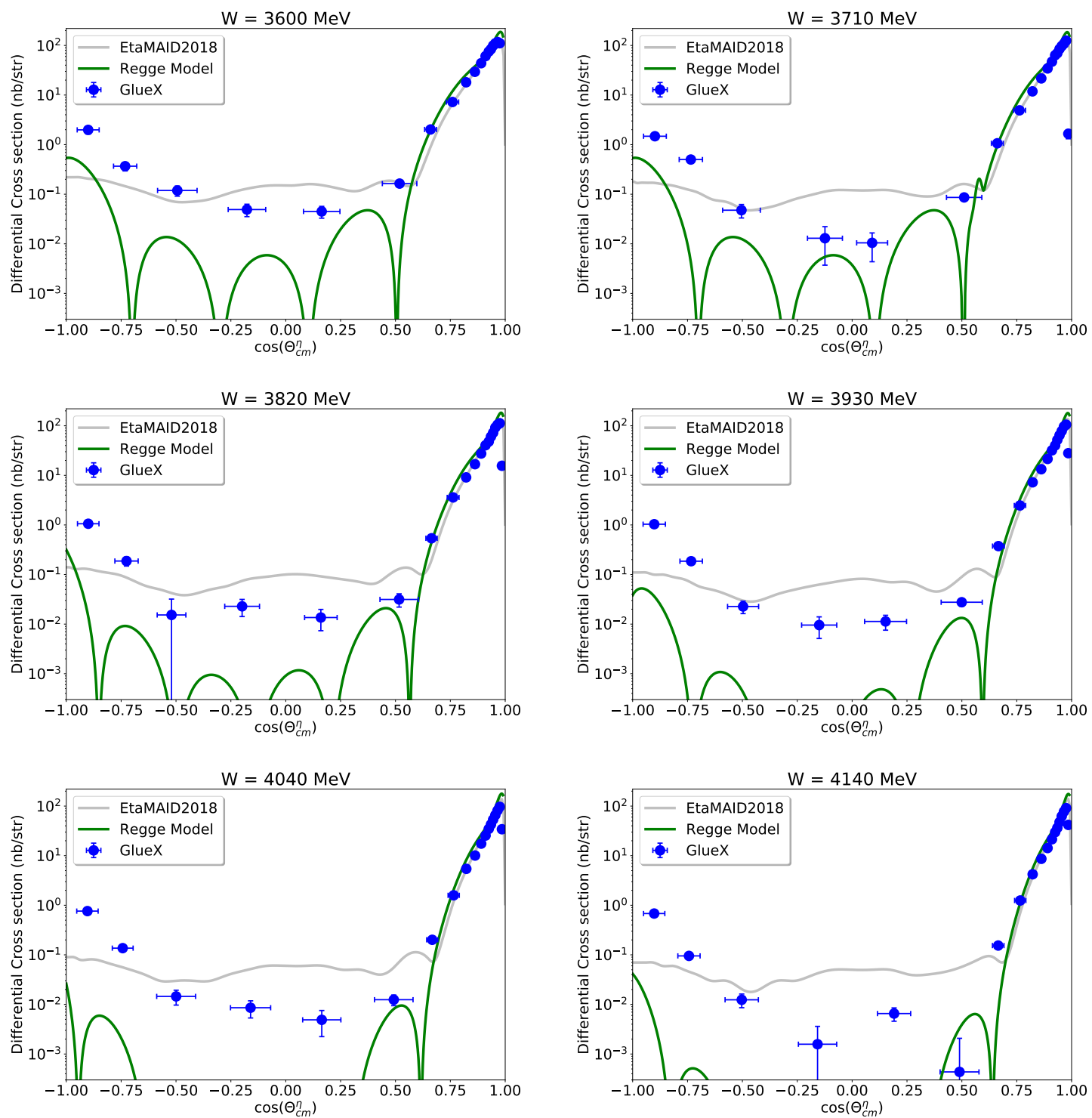

Figure 5.10: Differential cross section $\left(\frac{d \sigma}{d \Omega}\right)$ for 6 center of mass energy bins 3.60, 3.71, 3.82, 3.93, 4.04, and $4.14 \mathrm{GeV}$. Gluex data(blue), EtaMAID2018, and Regge theoretical models are in grey and green lines, respectively. 


\section{Differential Cross Section Results.}
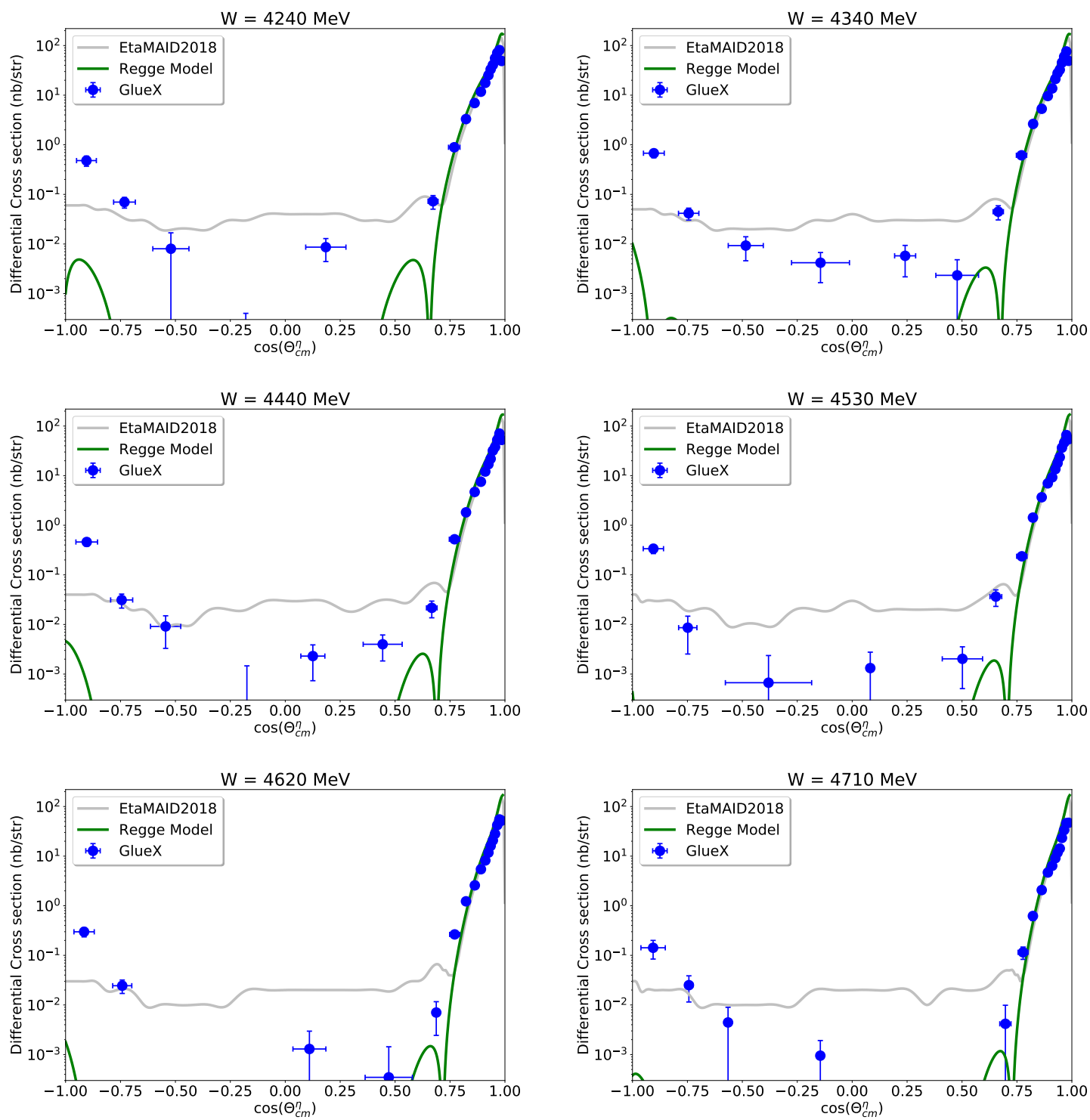

Figure 5.11: Same as 5.10 but for center of mass energy bins 4.24, 4.34, 4.44, 4.53, 4.62, and $4.71 \mathrm{GeV}$. 


\section{Differential Cross Section Results.}
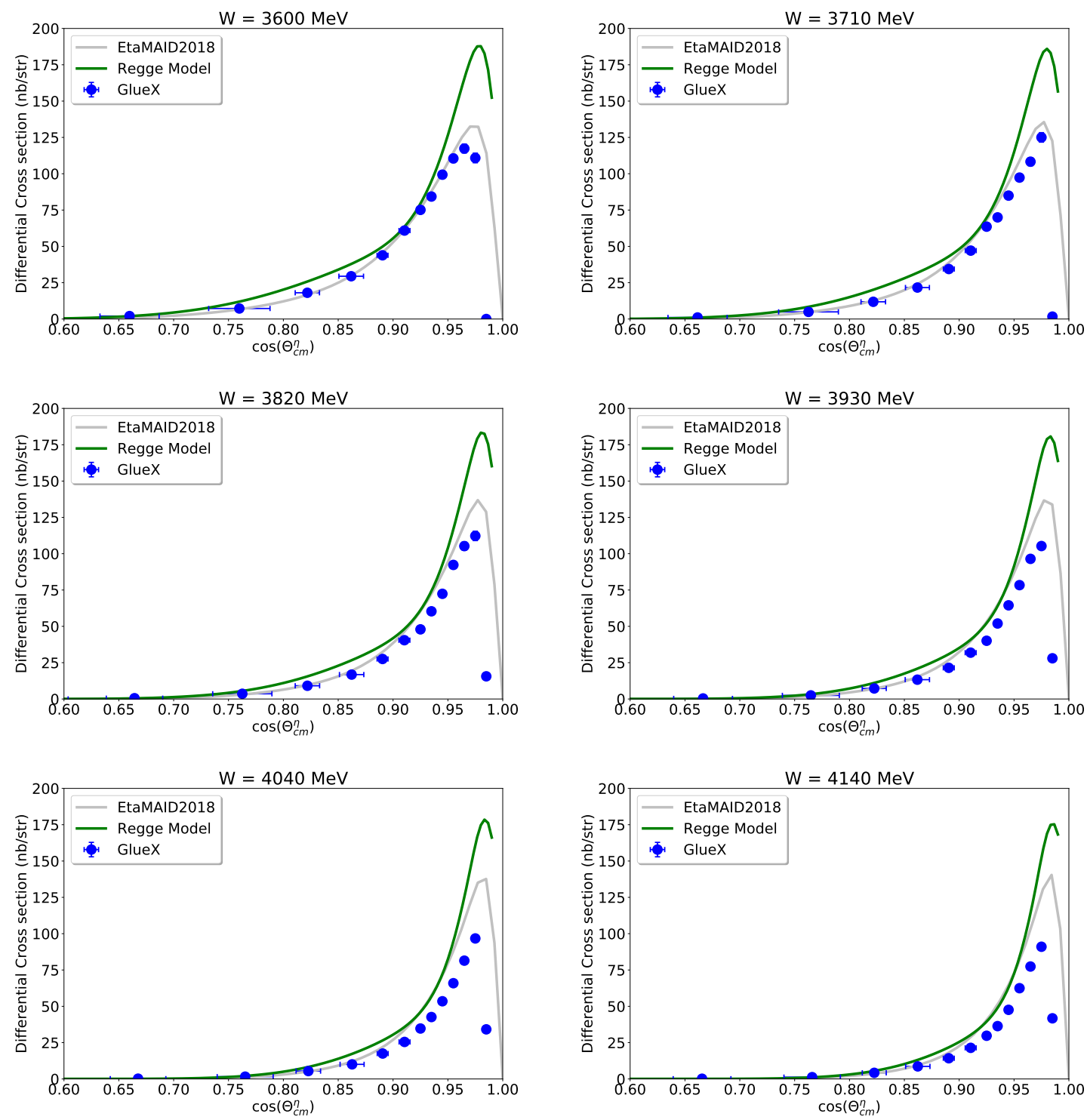

Figure 5.12: Differential cross section $\left(\frac{d \sigma}{d \Omega}\right)$ for $\cos \theta_{c m}^{\eta}>0.6$ in a linear scale for 6 center of mass energy bins 3.60, 3.71, 3.82, 3.93, 4.04, and $4.14 \mathrm{GeV}$. Gluex data(blue), EtaMAID2018, and Regge theoretical models are in grey and green lines, respectively. 


\section{Differential Cross Section Results.}
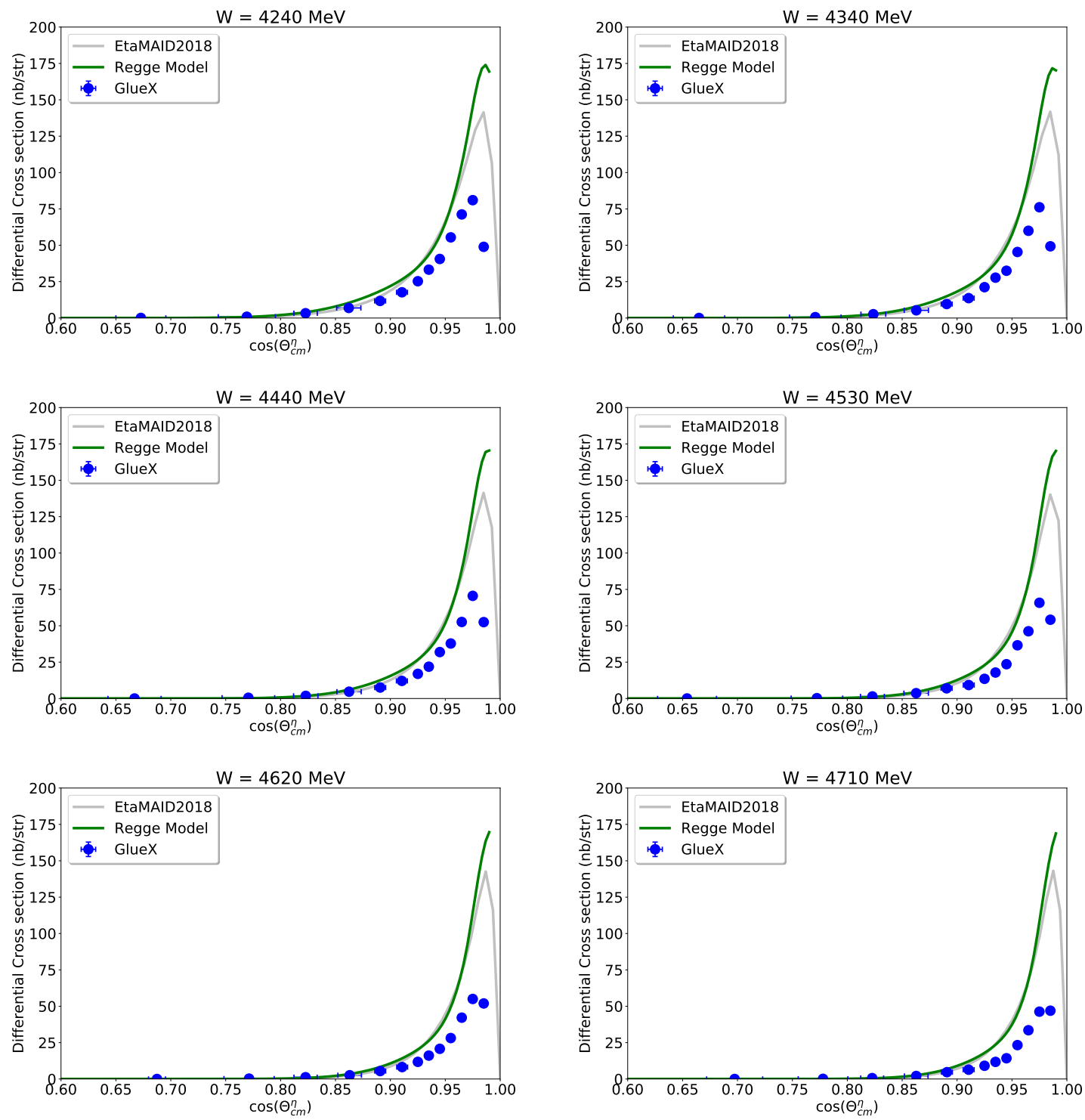

Figure 5.13: Same as 5.12 but for center of mass energy bins $4.24,4.34,4.44,4.53,4.62$, and $4.71 \mathrm{GeV}$. 
Weighted average differential cross section ratio
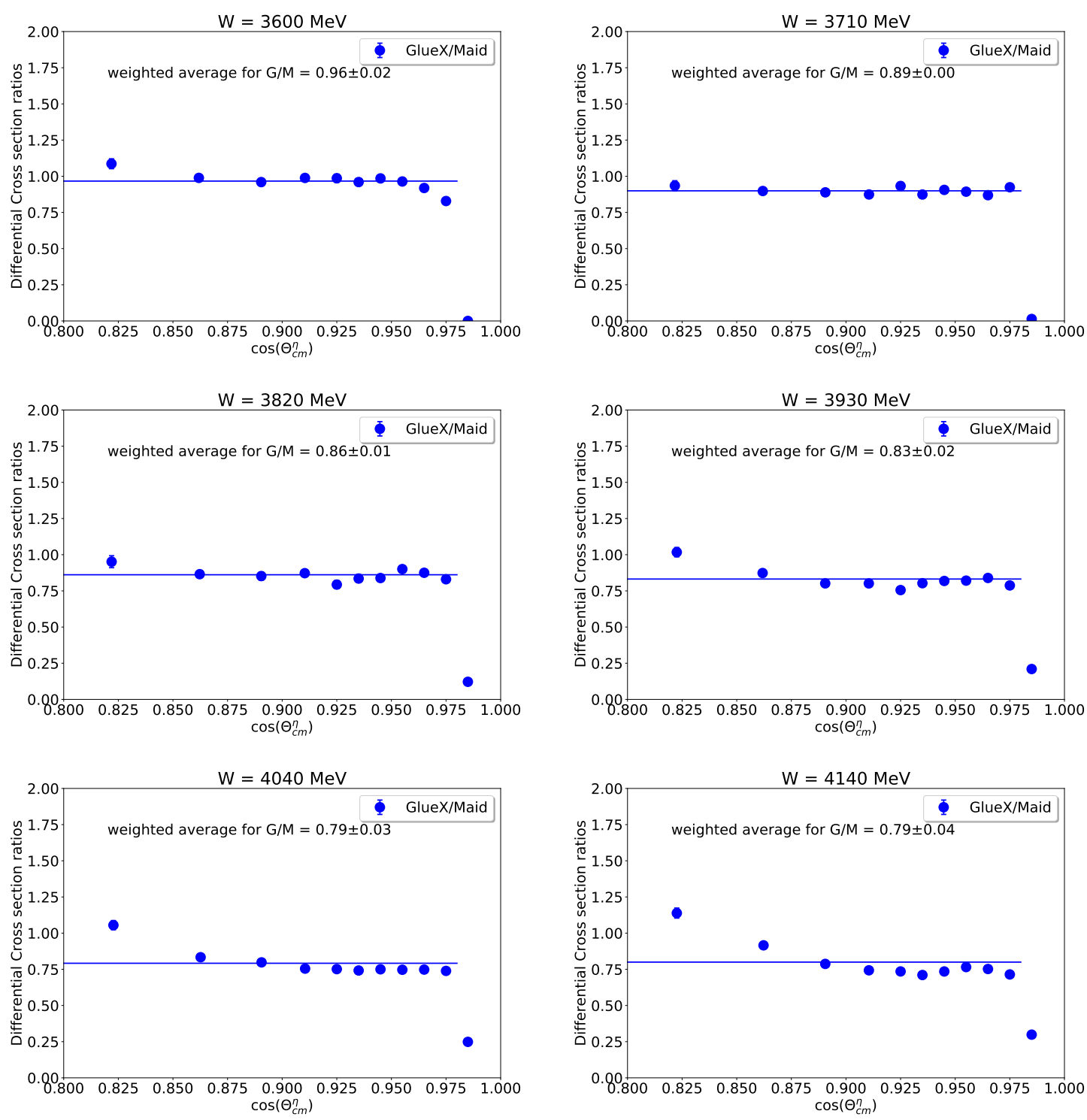

Figure 5.14: Weighted average differential cross section ratios between GlueX data and EtaMAID2018 model at $\cos \theta_{c m}^{\eta}>0.8$ for 6 center of mass energy bins $3.60,3.71,3.82,3.93$, 4.04 , and $4.14 \mathrm{GeV}$. 
Weighted average differential cross section ratio.
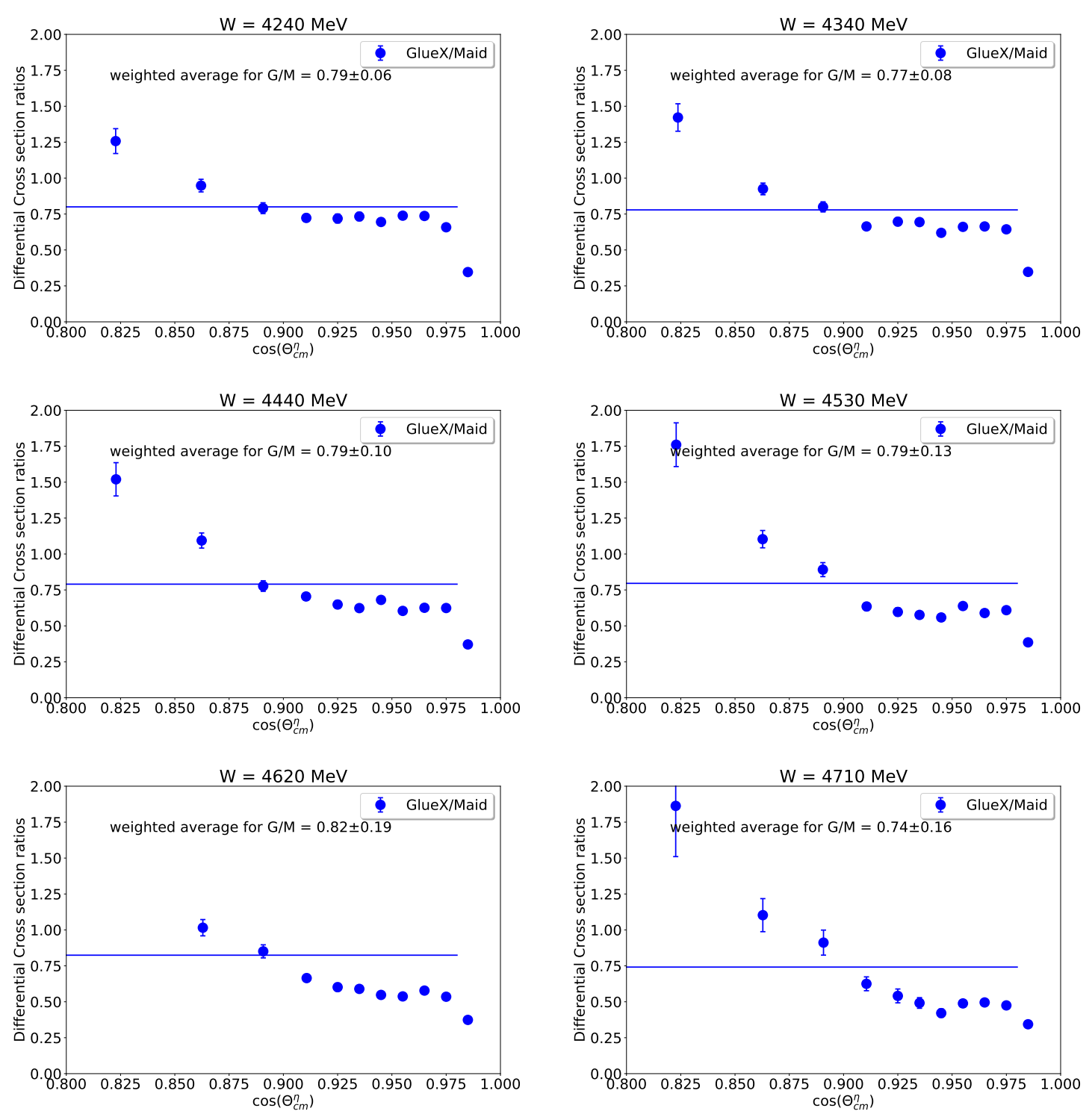

Figure 5.15: Same as Fig. 5.14. 


\subsection{Differential cross sections $(d \sigma / d t)$ for High Energy Data}

As for the low energy data differential cross sections $d \sigma / d t$ have also been determined. The high energy $\eta$ photo-production cross section as a function of -t are shown in figures 5.16, and 5.17. The GlueX data show the typical increase of the cross section at very large $|t|$ (low $\mathrm{u}$ ) up to $20 \mathrm{GeV}^{2}$. The new GlueX data at large $\mathrm{t}$ will provide new information to improve the understanding of the production mechanism of $\eta$ mesons.

At small $|t|<2 G e V^{2}$, the data follow roughly the shape of the EtaMAID2018 model. Figs. 5.18, and 5.19 show a linear fit for $\log (d \sigma / d t)$ over a $-\mathrm{t}$ range of $0.14<-t<1.0 \mathrm{GeV}^{2}$. In general, the fitted line is parallel to the predicted EtaMAID2018 cross sections up to W $=4.53 \mathrm{GeV}$, and within $20 \%$ of their values. At $\mathrm{W} \geq 4.62 \mathrm{GeV}$, the two lines are not parallel, and the there is no agreement between the data and the models. 
Differential Cross Section Results.
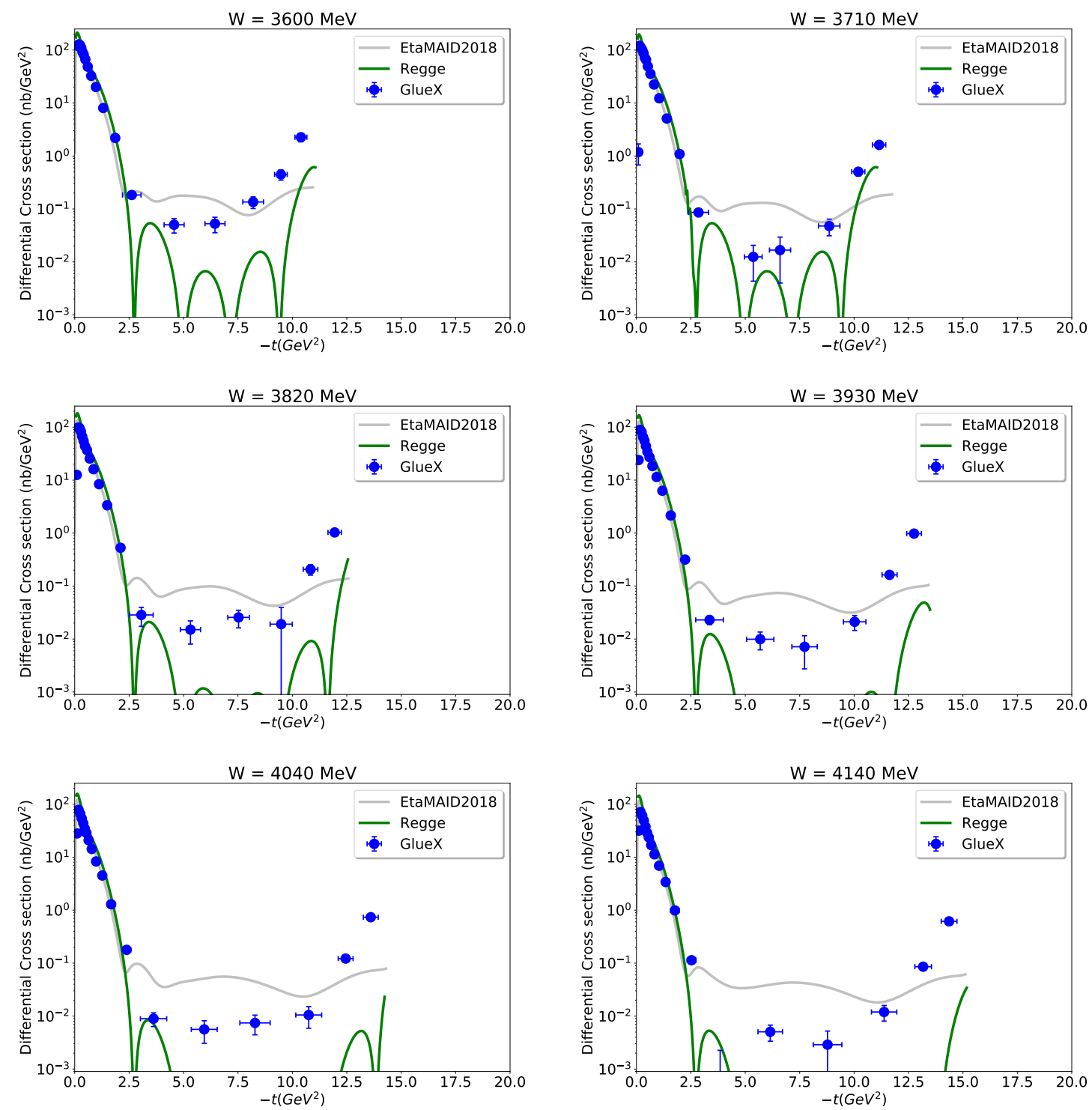

Figure 5.16: Differential cross section $\left(\frac{d \sigma}{d t}\right)$ for 6 center of mass energy bins 3.60, 3.71, 3.82, 3.93, 4.04, and $4.14 \mathrm{GeV}$ as a function of (-t). Gluex data(blue), EtaMAID2018 and Regge theoretical models are in grey and green lines, respectively. 
Differential Cross Section Results.
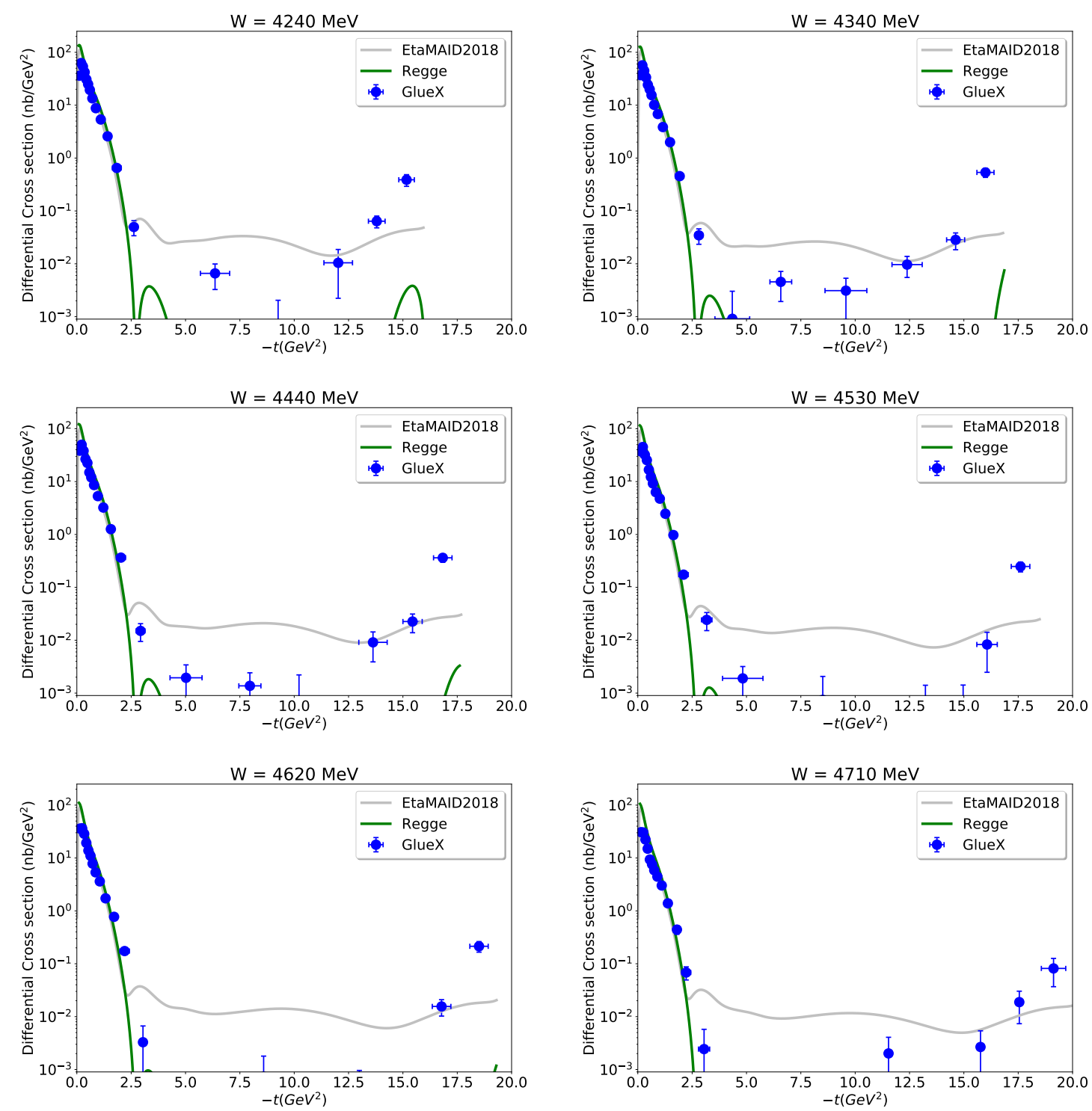

Figure 5.17: Same as Fig. 5.16 but for 6 center of mass energy bins 4.24, 4.34, 4.44, 4.53, 4.62, and $4.71 \mathrm{GeV}$. 
Differential Cross Section Results.
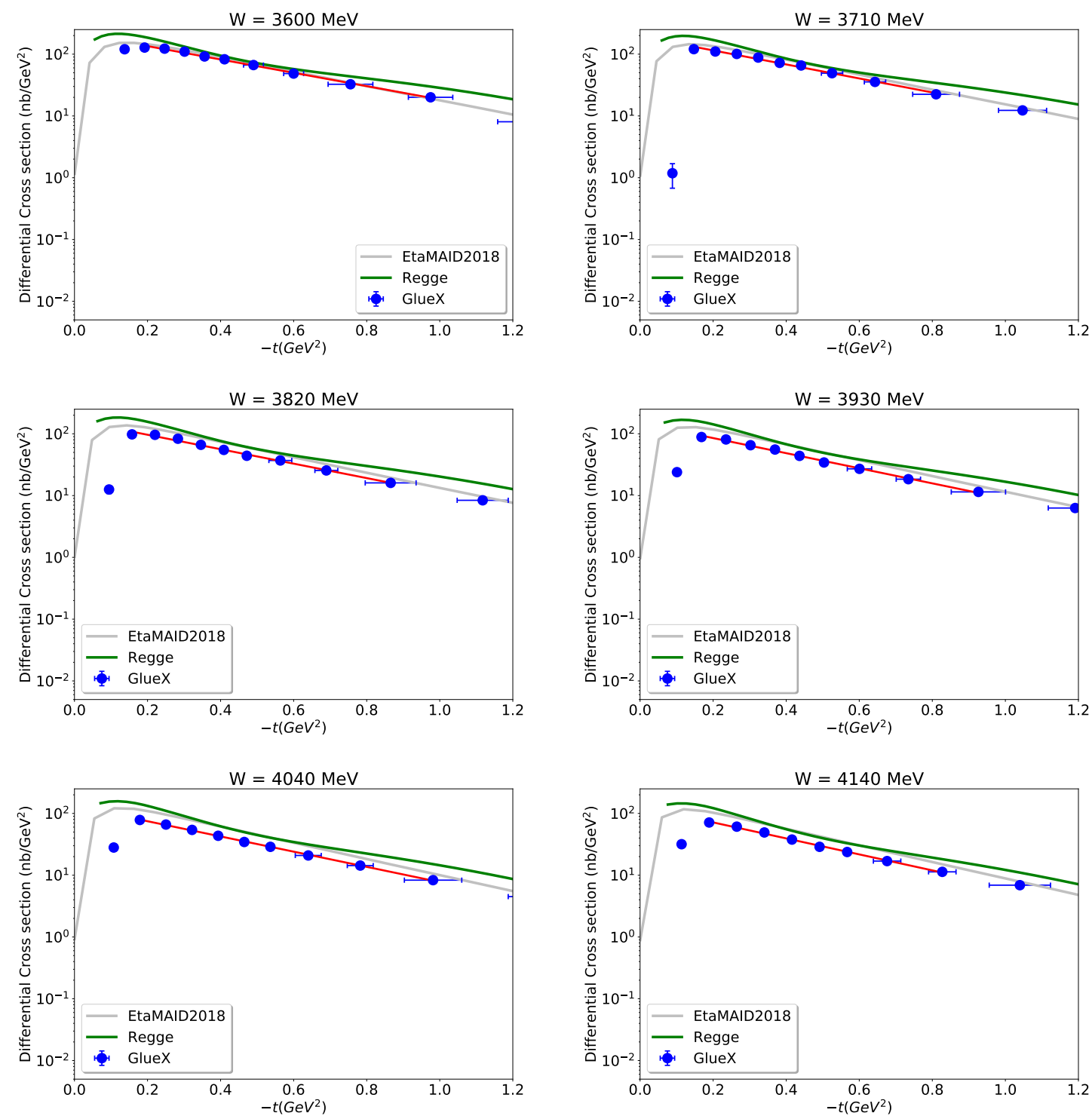

Figure 5.18: Differential cross section $\left(\frac{d \sigma}{d t}\right)$ for 6 center of mass energy bins 3.60, 3.71, 3.82, $3.93,4.04$, and $4.14 \mathrm{GeV}$ as a function of $(-\mathrm{t})$ for $-t<1.2 \mathrm{GeV}^{2}$. The red line is a linear fit for the GlueX data at $0.14<-t<1.0 \mathrm{GeV}^{2}$ 
Differential Cross Section Results.
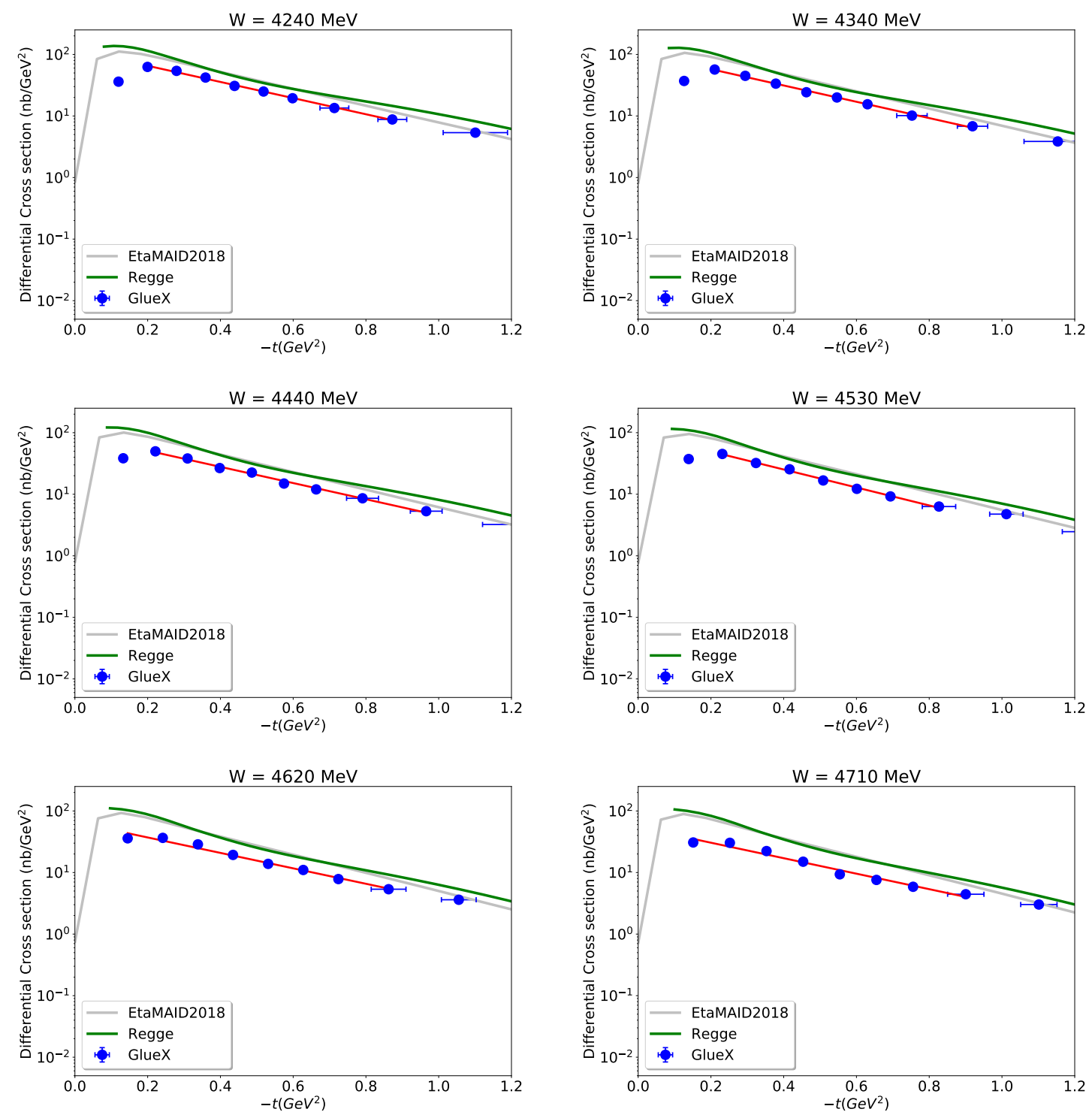

Figure 5.19: Same as Fig. 5.18 but for 6 center of mass energy bins 4.24, 4.34, 4.44, 4.53, 4.62 , and $4.71 \mathrm{GeV}$. 


\section{Extracted fit parameters}
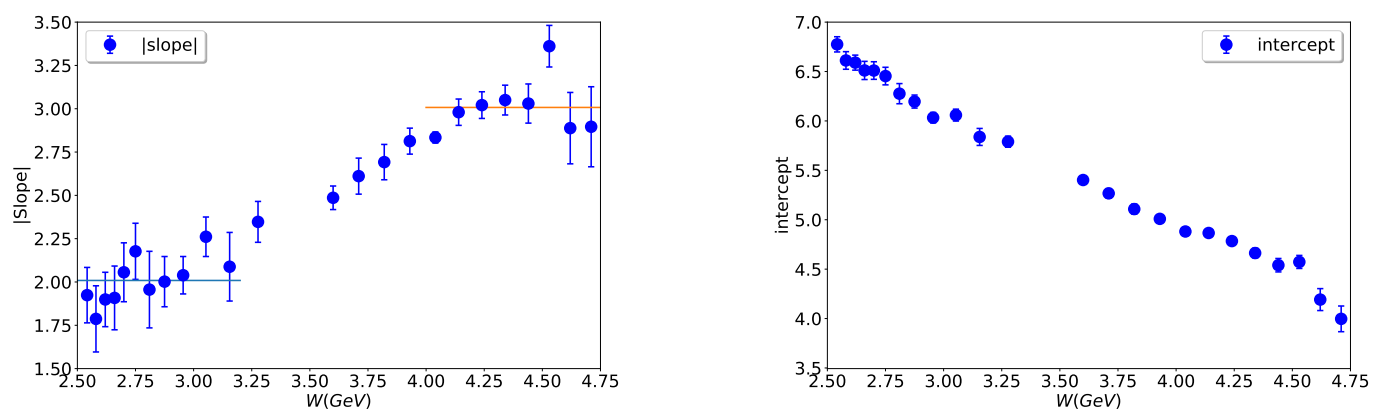

Figure 5.20: Absolute value of the slopes and intercepts extracted from each energy bin vs W.

\subsection{Combined $d \sigma / d t$ discussion for $2.54<W<4.8 \mathrm{GeV}$}

This section summarizes the results based on the discussion in section 1.4. Figs. 5.18, and 5.19 as well as the similar fits for the low energy data show a linear fit of $\log (d \sigma / d t)$ vs (-t) in a very small values of $0.14<-t<1 G e V^{2}$. The slope and intercept extracted from all the 24 energy bins are shown in Fig. 5.20.

The absolute value of the slope could be fit with two constants, one is for the low energy data $(2.01 \pm 0.05)$, and the other one is for $W>4 \mathrm{GeV}(3.01 \pm 0.07)$. This may indicate different exchanged Regge particle. The extrapolation of these linear fits to $t=t_{\text {min }}$, where $t_{\min }$ is very close to zero as shown in chapter 1 , are proportional to the total $\eta$ photoproduction cross section. Fig. 5.21 shows these extrapolated values in blue as well as the integrated (IC) total cross section calculated from the equation:

$$
I C=\int_{D A} \frac{d \sigma}{d \Omega} d \Omega
$$

where DA is the detector acceptance ( $97 \%$ for the low energy data, while it is $98 \%$ for the high energy data). Dividing the extraploated data by $\log (s)$, results in a total cross section $\sigma_{t}$ that is in agreement with the theoretical predictions from Byung-Geel $\mathrm{Yu}$ [57] at the low energy end $(\mathrm{W}<3.5 \mathrm{GeV})$. The IC is also in a good agreement with the Yu predictions for $\mathrm{W}<2.8 \mathrm{GeV}$. The ratios of the extrapolated $d \sigma / d t$ at $t=t_{m i n}$, and $\sigma_{t}$ with respect to 


\section{Total Cross Section}
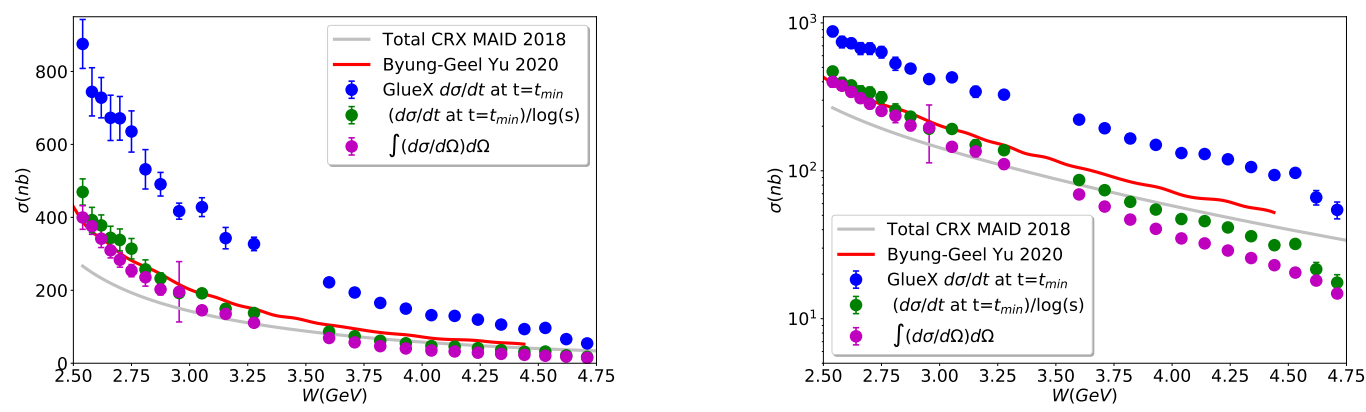

Figure 5.21: Combined total cross section in a linear (left), and log (right) scales from the low and high energy data sets Vs center of mass energy W.

the IC are shown in Fig. 5.22. The latter ratio is fitted with the constant green line. This fit show an agreement between the two experimentally calculated total cross sections (IC and $\left.\sigma_{t}\right)$ within $24 \pm 3 \%$.

The ratios of the experimental cross sections to the EtaMAID2018 total cross sections are shown in Fig. 5.23. There is a good agreement within $10 \%$ between GlueX $\left(\sigma_{t}\right)$ and EtaMAID2018 total cross section for $3.5<W<4.6 \mathrm{GeV}$. The ratio between these two total cross sections is $88 \pm 3 \%$ (the green line).

Comparing equation 1.37 to the straight line equation, one can calculate the value of $\alpha^{\prime}=\frac{\mid \text { slope } \mid}{C_{1}}$. The values of $\alpha^{\prime}$ for all $2.54<W<2.8 \mathrm{GeV}$ are shown in Fig. 5.24. These values have a constant fit of $0.50 \pm 0.01$. 


\section{Total Cross Section Ratios}

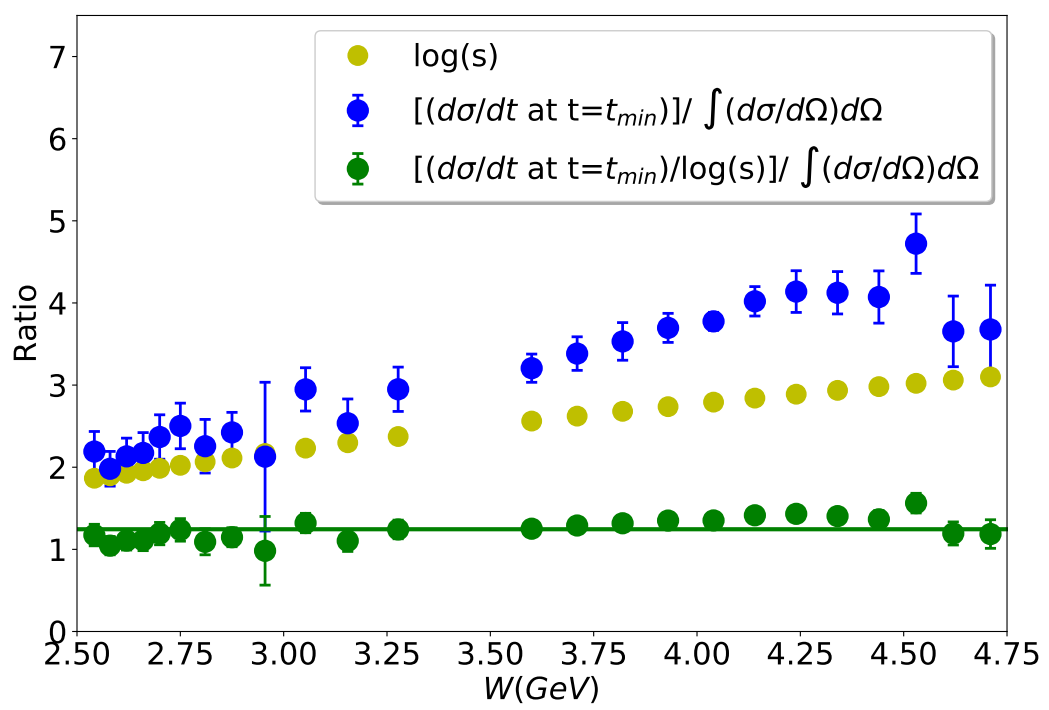

Figure 5.22: The ratios of the extrapolated cross section (blue) and $\sigma_{t}$ (green) with respect to the IC. The yellow points are the $\log (s)$ values.

\section{Total Cross Section Ratios}

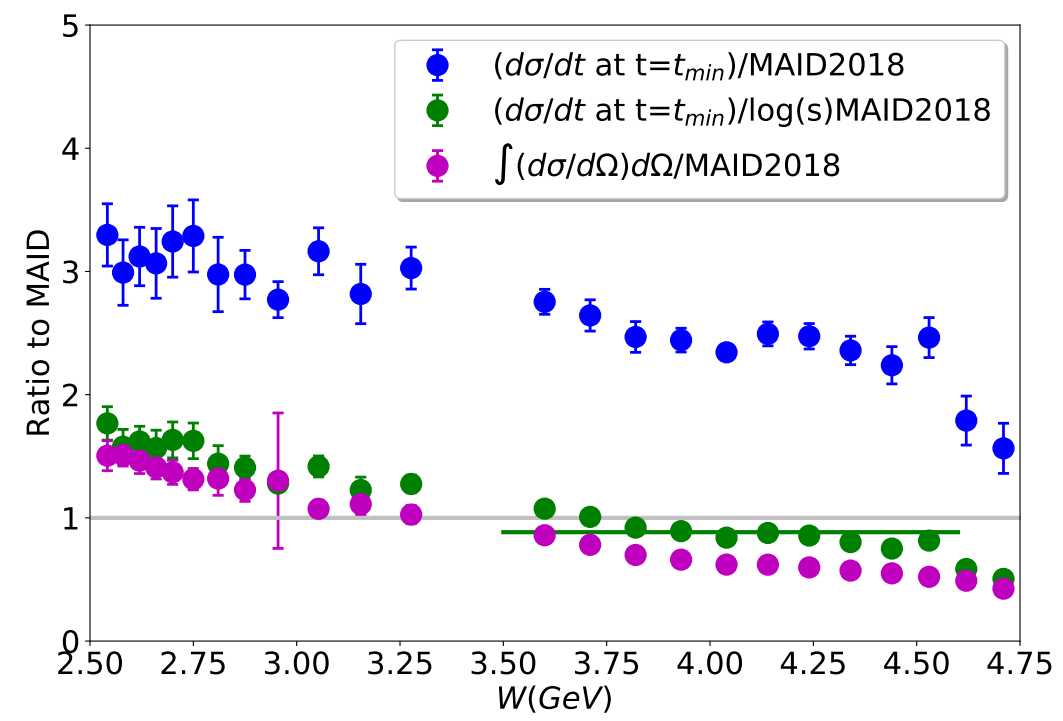

Figure 5.23: The ratios of the extrapolated cross section (blue), $\sigma_{t}$ (green), and IC (magenta) with respect to the EtaMAID2018 total cross section. 
$\alpha^{\prime}$ values of equation 1.37

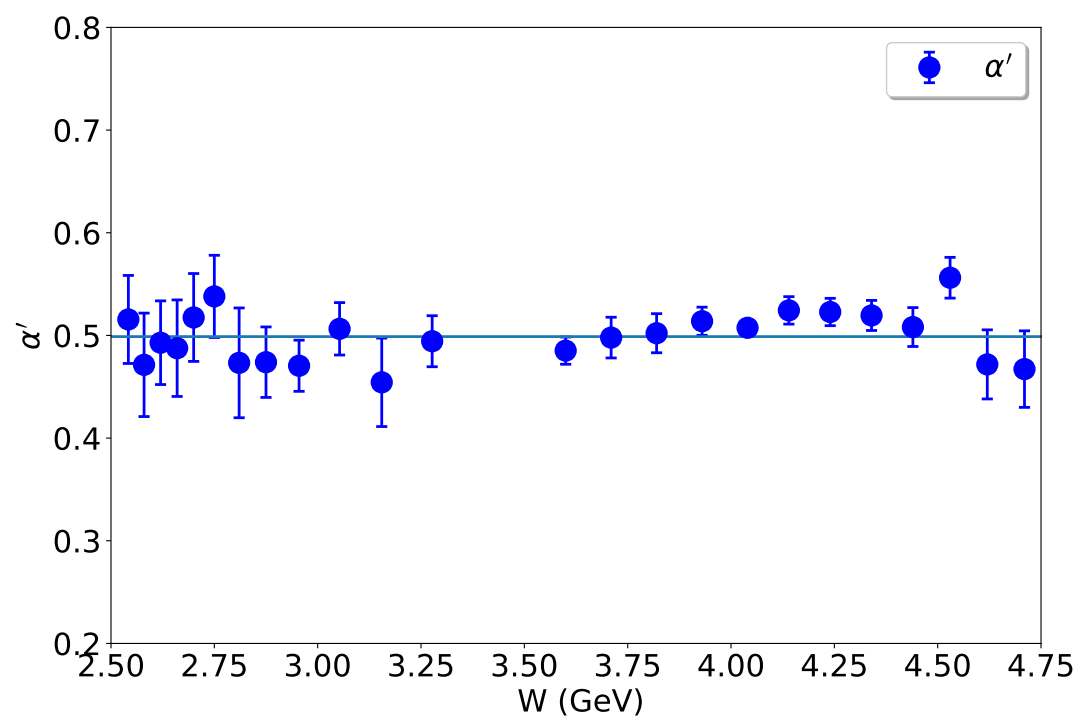

Figure 5.24: The values of $\alpha^{\prime}$ for all energy bins. The blue line is a linear fit of $0.50 \pm 0.01$ 


\section{CHAPTER 6}

\section{Summary and Conclusion}

The GlueX experiment is studying the light meson spectrum and searching for exotic and hybrid mesons. The GlueX start counter detector propagation time calibration procedure has been updated. In general, the effective speed of light measured from this calibration ranges from $13 \mathrm{~cm} / \mathrm{ns}$ to $25 \mathrm{~cm} / \mathrm{ns}$. The start counter efficiency has been calculated for each geometrical section, and for each scintillator paddle. The overall start counter efficiency has been determined to be $97.2 \pm 0.1 \%$. The start counter time resolution is well below the 350 ns, which allows for identifying individual electron beam bunches, sent by CEBAF at a frequency of $249.5 \mathrm{MHz}$, and the coincidence photon from which an event has been created. The start counter time resolution has been stable through a total run period of over 5 years with no clear sign of deterioration.

In this work, the reaction $\gamma p \rightarrow \eta p$ was used to test the Regge theory for $\mathrm{s}$, and t- dependence. The $\eta$ was identified by the decay $\eta \rightarrow \gamma \gamma$, which has a branching ratio of $39.41 \pm 0.20 \%$. The photo-production differential cross sections $(d \sigma / d \Omega)$ have been determined for a beam energy ranging from 2.9 up to $11.6 \mathrm{GeV}$. These results have been presented in 24 center of mass energy ( $\mathrm{W}=2.542 \mathrm{GeV}$ up to $\mathrm{W}=4.71 \mathrm{GeV}$ ) bins as a function of the cosine of the $\eta$ production polar angle in the $\eta-p$ center of mass frame. The new measurements are the most extensive high statistics data set covering a big range of $\mathrm{W}$. Two data sets have been used to determine the $\eta$ differential cross sections:

- The nominal GlueX high energy data with a beam energy range of $6.2 \mathrm{GeV}<E_{\gamma}<$ 11.6 GeV have been collected in Spring 2017.

- The low energy data set of $2.9 \mathrm{GeV}<E_{\gamma}<5.9 \mathrm{GeV}$ have been collected in Fall 2018. Differential cross sections $(d \sigma / d \Omega)$ of $\eta$ photo-production off the proton, from the low energy data set, have been compared with the overlapping CLAS data and the recent theoretical EtaMAID2018 model as well as the Regge cross section predictions by JPAC. The GlueX data are covering a larger $\theta_{c m}^{\eta}$ angle range than the CLAS data in both forward and backward directions. In general the new GlueX results are systematically higher than 
the CLAS measurements from 2009 like recent 2020 CLAS results. The GlueX results agree for $-0.8<\cos \theta_{c m}^{\eta}<-0.3$. The GlueX cross sections roughly follow the shape of the EtaMAID2018 model. They agree with the Regge model calculations within $10 \%$ for forward going $\eta$ 's with small angles $\Theta_{c m}^{\eta}<20^{\circ}$, while the EtaMAID2018 model underestimates these cross sections.

A detailed comparison between the experimental results and the theoretical EtaMAID2018 model has been performed for all W bins through this study. A good agreement has been found for small angles between the Gluex measurements and the Regge model. As W increases, a good agreement between the new data and the EtaMaid2018 model is not expected due to the dominance of t-channel processes of $\eta$ 's photo-production, while the model is an isobar model with a Regge background. At high energies, $\eta$ photo-production is mainly a t-channel process. The new high energy data can be used to constraint the production mechanisms within the theoretical model predictions, and a better agreement between data and theoretical model is expected in the future.

The $\frac{d \sigma}{d t}$ data have been fitted with an exponential for a small range of $0.14<-t<1$ $\mathrm{GeV}^{2}$. The extracted slope factor for the energy data $W<3.2 \mathrm{GeV}$ have a fitted value of $2.01 \pm 0.05$. This fitted value increased to $3.01 \pm 0.07$ for the high energy data $W>4.0 \mathrm{GeV}$. This indicates that there are two different exchanged particles at low and high energies. The integrated cross sections calculated from $(d \sigma / d \Omega)$ were compared to the extrapolated $\left(d \sigma / d t\right.$ at $\left.\mathrm{t}=t_{\min }\right) / \log (s)$, which is denoted $\sigma_{t}$. The two are in agreement within $20 \%$. Both experimental cross sections were also compared to the EtaMAID2018, and ByungGeel Yu 2020 total cross sections. The experimental cross sections agree with the ByungGeel Yu 2020 within 15\% at the low energy, while agree within same percentage with the EtaMAID2018 at the high energy data.

In the appendix A, a first look at the photo-production of $\eta^{\prime}$ has been presented with its radiative decay branch $\left(\pi^{+} \pi^{-} \gamma\right)$, with a branching ratio of $29 \%$. This branch was chosen since it has the lowest number of detected final state particles. The background in this channel was very challenging. Therefore understanding the yield outcomes has been compared to the highest branching ratio decay $\left(\pi^{+} \pi^{-} \eta\right)$, with $42 \%$. The $\eta$ decay has also 
been identified with the decay into $\gamma \gamma$ with a branching ratio of $39.4 \%$. The ratio of the $\eta^{\prime}$ yield has been determined from the two decay channels and compared to the expected value of 1.72 from the PDG. They are in agreement within $12 \%$. 


\section{Appendix}

In this appendix, a summary of $\eta^{\prime}$ photo-production analysis will be presented as well as differential cross section tables for $\eta$ photo-production with systematic error calculations for the GlueX low energy data set.

\section{Photo-production of $\eta^{\prime}$ Mesons}

Photo-production of $\eta^{\prime}$ mesons is an important process since it is one of the decay products of the potential exotic candidate $\pi_{1}(1600) . \quad \eta^{\prime}$ mesons are pseudoscaler particles like $\eta$ mesons. According to flavor symmetry SU(3), both particles would have similar masses if the strange quark had the same mass as the up and down quarks. However the strange quark is considerably heavier, resulting in an $\eta^{\prime}$ mass of $0.958 \mathrm{GeV}$.

There are two main $\eta^{\prime}$ decay channels. The first one is $\eta^{\prime} \rightarrow \pi^{+} \pi^{-} \eta$ with a branching ratio $42.6 \pm 0.70 \%$, and the $\eta$ is identified via the decay $\eta \rightarrow \gamma \gamma$ as in chapter 3 . The second common decay mode of the $\eta^{\prime}$ is $\eta^{\prime} \rightarrow \pi^{+} \pi^{-} \gamma$ with a branching ratio of $28.9 \pm 0.5 \%$. We analyzed this decay mode first. This channel is very challenging due to a large background under the invariant $\eta^{\prime}$ mass peak and a large peak in the invariant mass spectrum near the $\rho$ mass due to a superposition of a $1 \%$ branching ratio of the $\rho$ decaying to $\pi^{+} \pi^{-} \gamma$, and the decay $\omega \rightarrow \pi^{+} \pi^{-} \pi^{0}$ with a missing undetected photon from one of the $\pi^{0}$, decay. Because of this challenging background, $\eta^{\prime}$ decay to $\pi^{+} \pi^{-} \eta$ has been analyzed as well. This allows one to evaluate and study the single photon and double photons detection efficiency. The yield ratio of the two $\eta^{\prime}$ decay branches have been determined and found in agreement with the PDG value within $10 \%$.

The invariant mass histogram of the $\pi^{+} \pi^{-} \gamma$ channel is shown in Fig. 1. The signal to background ratio is about 1:4. One way to improve the signal to background ratio in this channel is to apply a linear cut as indicated in the two dimensional Dalitz plot of $M_{M}^{2}\left(\pi^{-} \gamma\right)$ vs $M_{M}^{2}\left(\pi^{+} \gamma\right)$. Fig. 2 shows the comparison of dalitz plot in data and simulation. In data we found a large number of low energy photons due to hadronic split off in the calorimeter near the bottom left corner of the figure. The MC simulation using the standard GlueX 


\section{$\pi^{+} \pi^{-} \gamma$ invariant mass.}

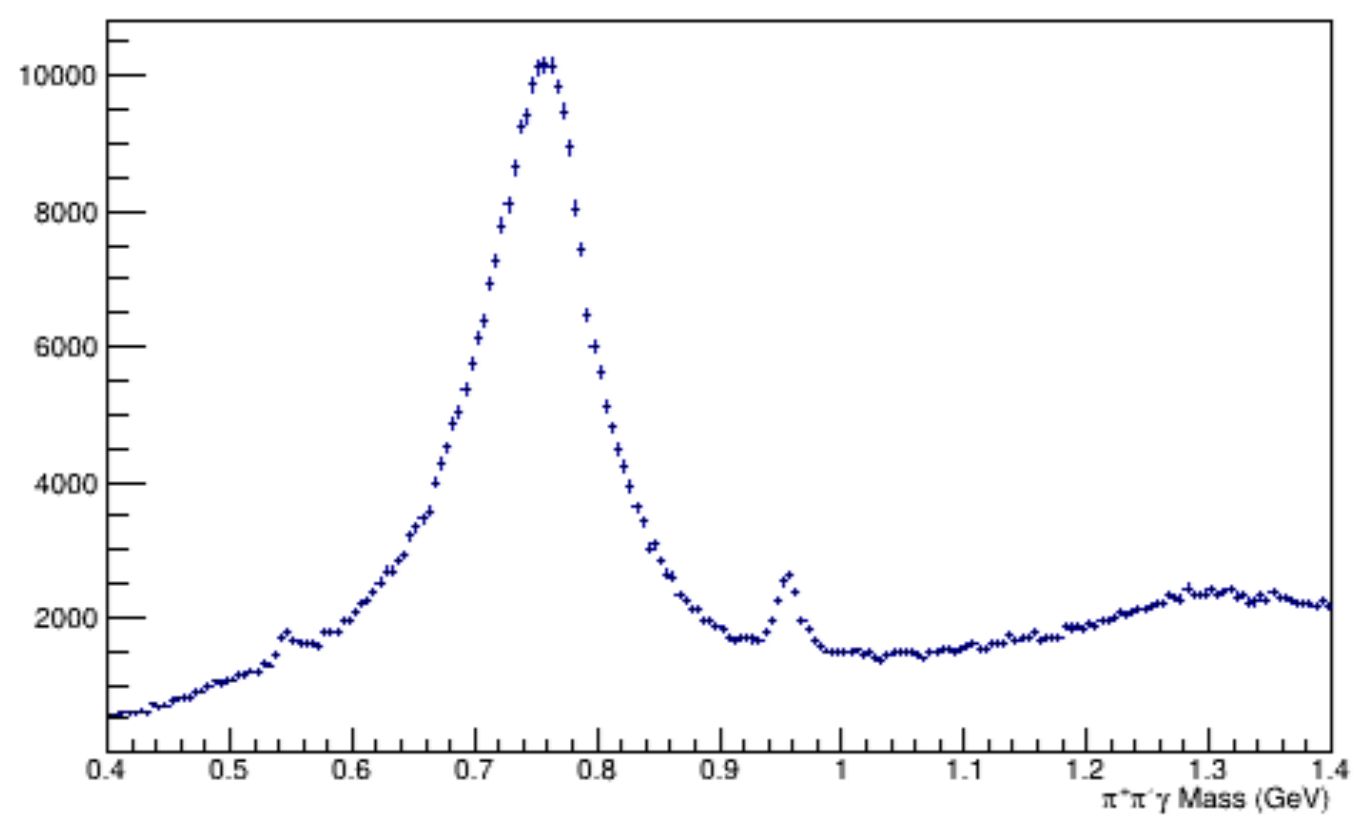

Figure 1: $\pi^{+} \pi^{-} \gamma$ invariant mass before applying Dalitz cut.

inclusive $\eta / \eta^{\prime}$ generator as in chapter 3 , does not show this structure. By applying this cut in the simulation, results in a signal loss of only $3 \%$. The Dalitz plot after the cut are shown in Fig. 3 for data and simulation. The signal to background ratio after the cut is now almost 1:1 as shown in Fig. 4. Also a clear $\eta$ peak decaying to $\pi^{+} \pi^{-} \gamma$ with $4 \%$ branching ratio is obtained.

We also analyzed the $\pi^{+} \pi^{-} \eta$ decay channel, where the $\eta$ is identified via the two decay photons. An integrated cross section for $\eta^{\prime}$ photo-production has been calculated for 10 beam energy bins at an energy range from $3 \mathrm{GeV}$ to $6 \mathrm{GeV}$. In each bin the $\eta^{\prime}$ yield is calculated from the radiative decay by fitting a Gaussian signal and either quadratic or linear background. The $\eta^{\prime}$ yield for the charged decay, where a very clean signal is obtained, is extracted by fitting a Gaussian and an exponential background. The yield comparison from the two channels is shown in Fig. 5 .

The relative branching ratio $R$ of the $\eta^{\prime}$ decay to $\pi^{+} \pi^{-} \gamma$ and to $\pi^{+} \pi^{-} \eta$ is calculated in equation 1 , which is taking into consideration that the $\eta$ is identified by $\gamma \gamma$ decay, which 


\section{Dalitz Plot Before Cut.}
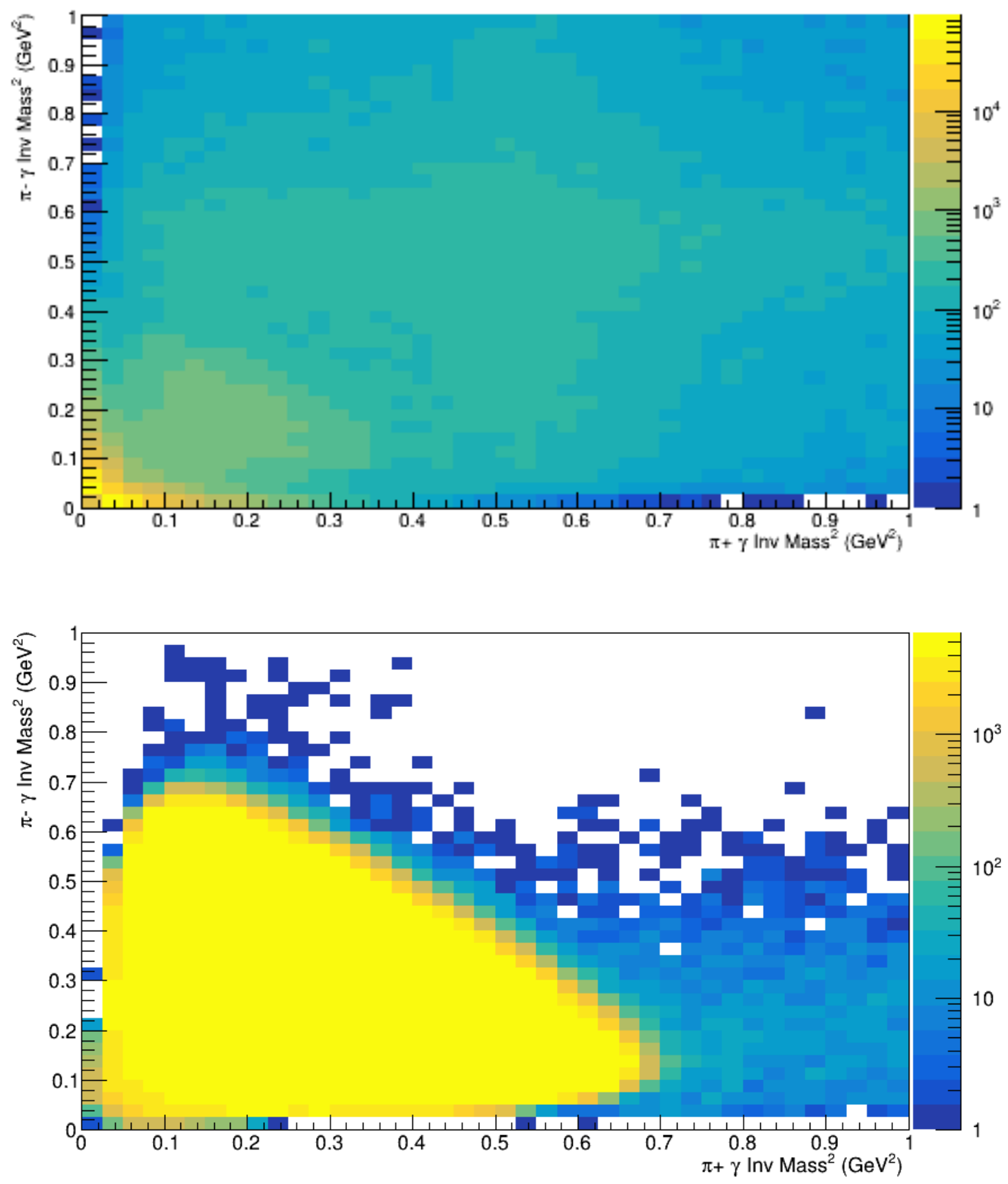

Figure 2: Invariant mass squared of $\pi^{-} \gamma \operatorname{vs} \pi^{+} \gamma$ for data (top) and simulation (bottom). 
Dalitz plots after cut.
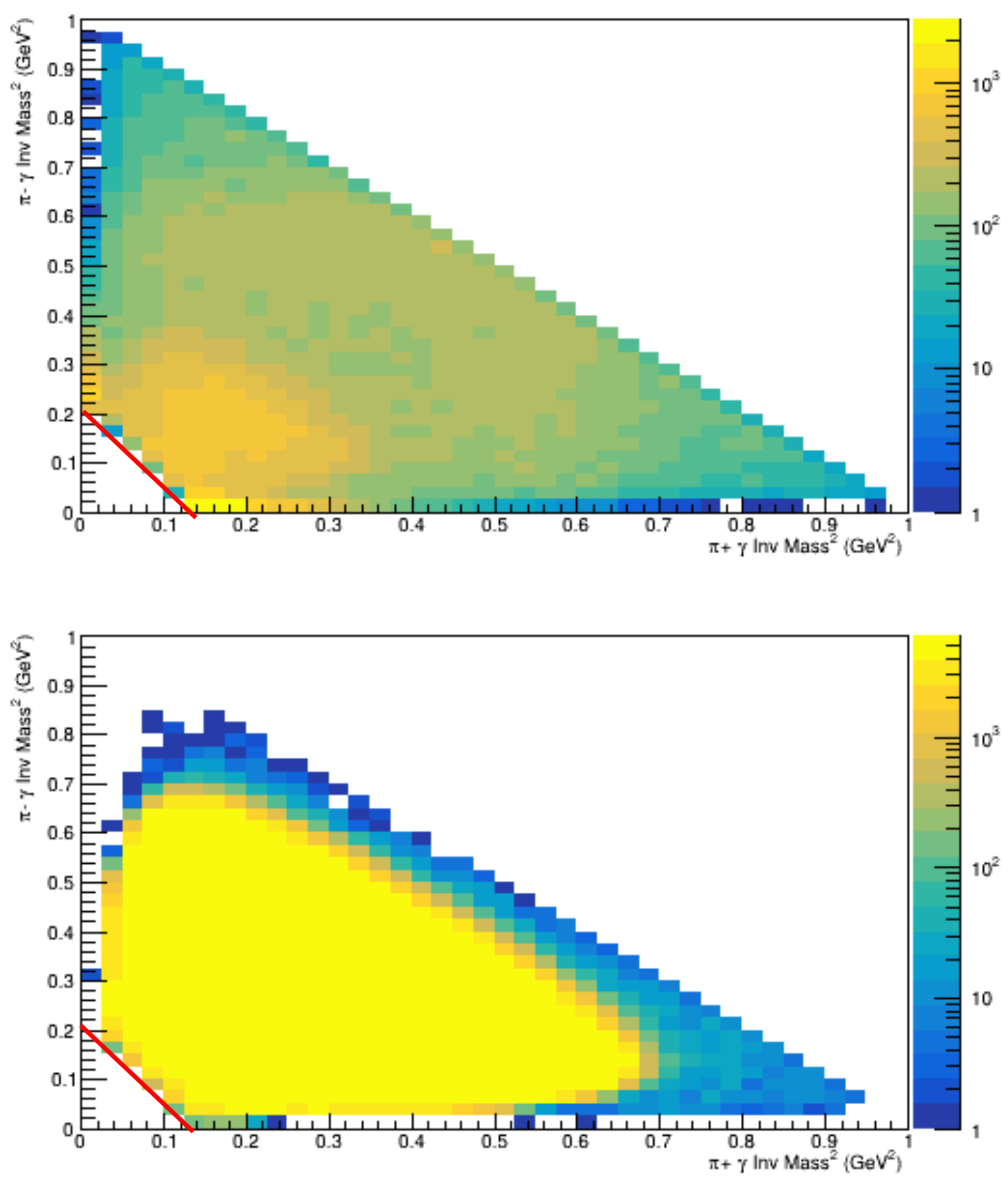

Figure 3: Invariant mass squared of $\pi^{-} \gamma \mathrm{vs} \pi^{+} \gamma$ for data (top) and simulation (bottom) after 2D Dalitz cut. 


\section{$\pi^{+} \pi^{-} \gamma$ invariant mass.}

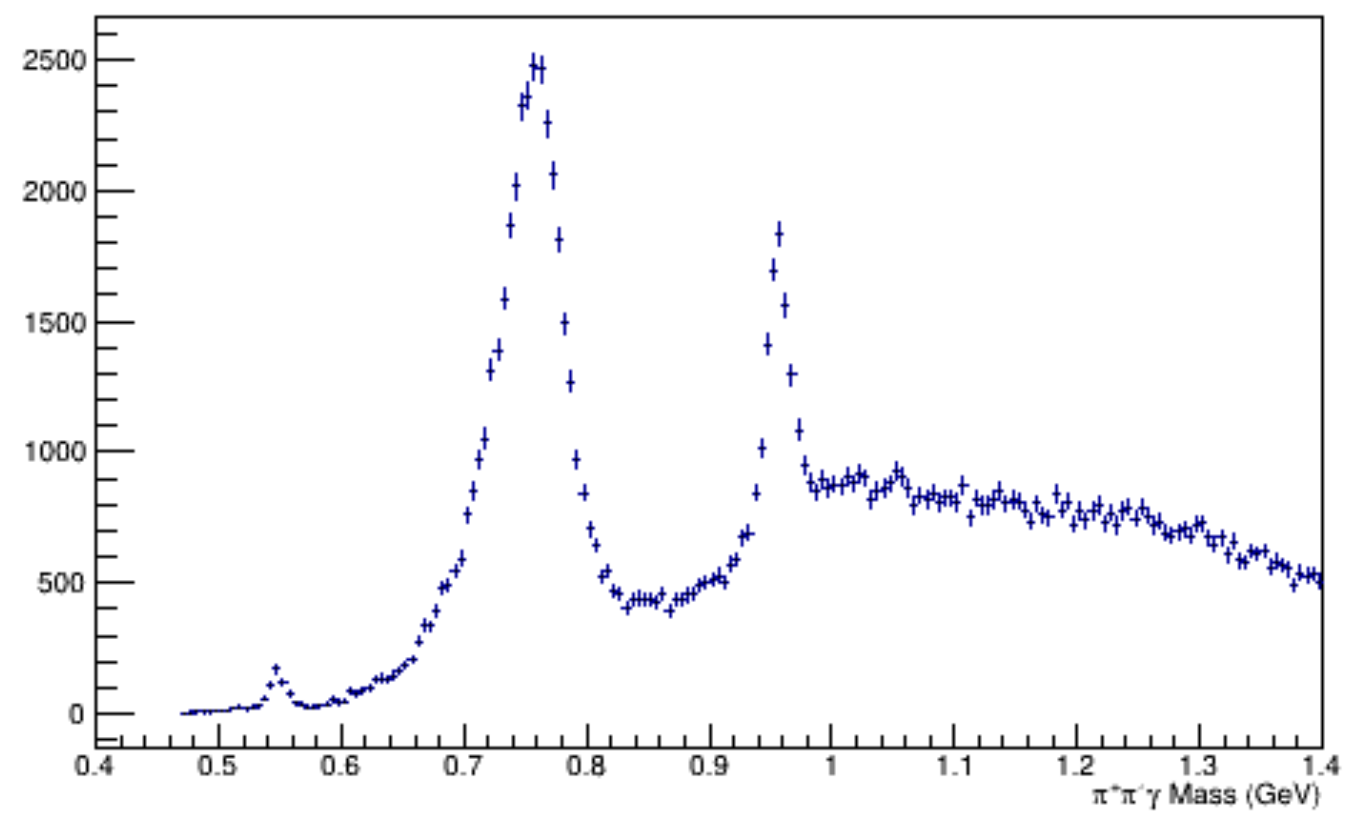

Figure 4: $\pi^{+} \pi^{-} \gamma$ invariant mass after applying Dalitz cut.

has a branching ratio of $39.41 \%$ itself. This ratio $R$ has been determined experimentally from the ratio of the flux normalized yields of both channels. The PDG calculated $R$ and the experimental one from the Fig. 5 of $(1.51 \pm 0.08)$ are in agreement within $12 \%$.

$$
R=\frac{B R_{\eta^{\prime} \rightarrow \pi^{+} \pi^{-} \gamma}}{B R_{\eta^{\prime} \rightarrow \pi^{+} \pi^{-} \eta} \times B R_{\eta \rightarrow \gamma \gamma}}=1.72 \pm 0.05
$$



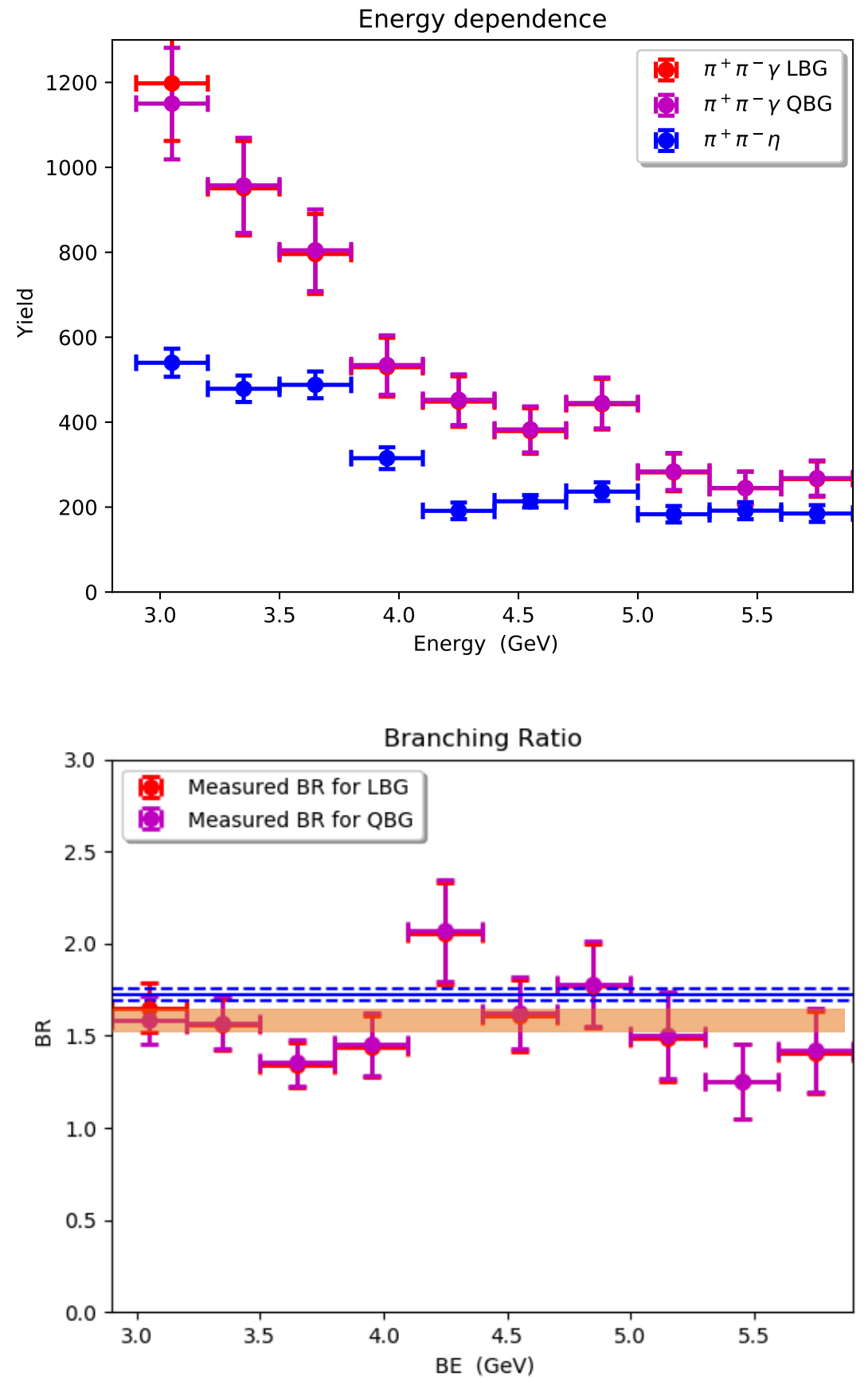

Figure 5: (Top) $\pi^{+} \pi^{-} \gamma$ yield with linear (quadratic) background in red (magenta). The yield of $\pi^{+} \pi^{-} \eta$ is in blue. (bottom) Experimental branching ratio fit(yellow box) and comparison to the PDG value with its error (blue solid and dashed lines). 


\section{Low Energy $\eta$ Cross section tables}

\begin{tabular}{|c|c|c|c|}
\hline $\cos (\theta)$ & CRX $($ nb/str $)$ & statistical error & sys err \\
\hline-0.893 & 11.37 & 2.05 & 2.42 \\
-0.712 & 5.63 & 1.59 & 1.88 \\
-0.459 & 2.48 & 0.82 & 0.97 \\
-0.137 & 7.06 & 0.90 & 1.06 \\
0.087 & 17.10 & 1.42 & 1.68 \\
0.301 & 15.77 & 1.41 & 1.66 \\
0.439 & 24.24 & 2.40 & 2.83 \\
0.543 & 39.09 & 3.09 & 3.65 \\
0.643 & 67.81 & 3.91 & 4.61 \\
0.741 & 94.13 & 4.76 & 5.62 \\
0.809 & 146.84 & 9.53 & 11.25 \\
0.850 & 167.88 & 10.11 & 11.93 \\
0.892 & 187.68 & 11.19 & 13.20 \\
0.920 & 218.50 & 18.13 & 21.39 \\
0.939 & 194.40 & 17.99 & 21.23 \\
\hline
\end{tabular}

Table 1: $\eta$ differential cross section for center of mass energy $=2542 \mathrm{MeV}$ 


\begin{tabular}{|c|c|c|c|}
\hline $\cos (\theta)$ & CRX $($ nb/str $)$ & statistical error & sys $s_{\text {err }}$ \\
\hline-0.903 & 13.49 & 1.56 & 1.73 \\
-0.717 & 7.54 & 1.10 & 1.22 \\
-0.452 & 1.97 & 0.57 & 0.63 \\
-0.134 & 7.14 & 0.60 & 0.67 \\
0.090 & 11.12 & 0.82 & 0.91 \\
0.298 & 12.94 & 0.90 & 1.00 \\
0.445 & 24.65 & 1.65 & 1.83 \\
0.545 & 39.35 & 2.07 & 2.30 \\
0.644 & 59.88 & 2.63 & 2.92 \\
0.742 & 89.33 & 3.25 & 3.61 \\
0.810 & 142.20 & 6.49 & 7.20 \\
0.850 & 154.12 & 6.84 & 7.59 \\
0.890 & 180.66 & 7.64 & 8.48 \\
0.919 & 173.75 & 11.25 & 12.49 \\
0.940 & 212.54 & 13.30 & 14.76 \\
0.955 & 4.07 & 2.87 & 3.19 \\
\hline
\end{tabular}

Table 2: $\eta$ differential cross section for center of mass energy $=2580 \mathrm{MeV}$

\begin{tabular}{|c|c|c|c|}
\hline $\cos (\theta)$ & CRX $($ nb/str $)$ & statistical error & sys $s_{\text {err }}$ \\
\hline-0.889 & 12.19 & 1.81 & 2.35 \\
-0.724 & 4.22 & 0.99 & 1.29 \\
-0.465 & 2.87 & 0.62 & 0.81 \\
-0.143 & 5.27 & 0.61 & 0.79 \\
0.080 & 8.37 & 0.83 & 1.08 \\
0.300 & 11.11 & 0.93 & 1.21 \\
0.445 & 19.43 & 1.69 & 2.20 \\
0.541 & 33.37 & 2.20 & 2.86 \\
0.644 & 51.75 & 2.71 & 3.52 \\
0.743 & 83.41 & 3.58 & 4.65 \\
0.811 & 126.79 & 6.99 & 9.09 \\
0.850 & 157.27 & 7.87 & 10.23 \\
0.890 & 168.20 & 8.55 & 11.12 \\
0.920 & 180.36 & 12.86 & 16.72 \\
0.940 & 203.78 & 14.24 & 18.51 \\
0.955 & 28.46 & 10.54 & 13.70 \\
\hline
\end{tabular}

Table 3: $\eta$ differential cross section for center of mass energy $=2620 \mathrm{MeV}$ 


\begin{tabular}{|c|c|c|c|}
\hline $\cos (\theta)$ & CRX $($ nb/str $)$ & statistical error & sys $s_{\text {err }}$ \\
\hline-0.887 & 10.30 & 1.52 & 1.73 \\
-0.726 & 3.43 & 0.84 & 0.96 \\
-0.462 & 2.61 & 0.52 & 0.59 \\
-0.151 & 4.66 & 0.50 & 0.57 \\
0.090 & 6.80 & 0.65 & 0.74 \\
0.294 & 6.86 & 0.71 & 0.81 \\
0.440 & 17.44 & 1.43 & 1.63 \\
0.545 & 27.41 & 1.76 & 2.01 \\
0.646 & 44.89 & 2.30 & 2.62 \\
0.744 & 78.15 & 3.07 & 3.50 \\
0.810 & 124.95 & 6.17 & 7.03 \\
0.851 & 140.65 & 6.75 & 7.69 \\
0.891 & 156.40 & 7.19 & 8.20 \\
0.920 & 181.80 & 11.63 & 13.26 \\
0.940 & 182.83 & 12.44 & 14.18 \\
0.955 & 73.56 & 11.96 & 13.63 \\
\hline
\end{tabular}

Table 4: $\eta$ differential cross section for center of mass energy $=2660 \mathrm{MeV}$

\begin{tabular}{|c|c|c|c|}
\hline $\cos (\theta)$ & CRX $($ nb/str $)$ & statistical error & sys $s_{\text {err }}$ \\
\hline-0.894 & 9.18 & 1.45 & 1.54 \\
-0.728 & 3.30 & 0.85 & 0.90 \\
-0.450 & 1.26 & 0.50 & 0.53 \\
-0.154 & 3.52 & 0.43 & 0.46 \\
0.091 & 5.56 & 0.59 & 0.63 \\
0.298 & 5.81 & 0.63 & 0.67 \\
0.444 & 14.57 & 1.34 & 1.42 \\
0.544 & 21.64 & 1.65 & 1.75 \\
0.644 & 38.91 & 2.22 & 2.35 \\
0.746 & 67.52 & 2.93 & 3.11 \\
0.811 & 107.29 & 5.87 & 6.22 \\
0.851 & 138.95 & 6.66 & 7.06 \\
0.891 & 164.84 & 7.65 & 8.11 \\
0.920 & 180.35 & 11.85 & 12.56 \\
0.940 & 177.84 & 12.17 & 12.90 \\
0.955 & 115.24 & 14.79 & 15.68 \\
\hline
\end{tabular}

Table 5: $\eta$ differential cross section for center of mass energy $=2700 \mathrm{MeV}$ 


\begin{tabular}{|c|c|c|c|}
\hline $\cos (\theta)$ & CRX $($ nb/str $)$ & statistical error & sys $s_{\text {err }}$ \\
\hline-0.894 & 8.51 & 1.59 & 1.86 \\
-0.718 & 4.30 & 0.80 & 0.94 \\
-0.451 & 1.74 & 0.38 & 0.44 \\
-0.162 & 2.61 & 0.34 & 0.40 \\
0.091 & 3.32 & 0.43 & 0.50 \\
0.297 & 3.70 & 0.47 & 0.55 \\
0.443 & 12.18 & 1.02 & 1.19 \\
0.543 & 16.74 & 1.28 & 1.50 \\
0.645 & 30.40 & 1.66 & 1.94 \\
0.744 & 60.43 & 2.35 & 2.75 \\
0.811 & 95.47 & 4.71 & 5.51 \\
0.851 & 120.91 & 5.41 & 6.33 \\
0.891 & 158.90 & 6.33 & 7.41 \\
0.920 & 165.85 & 9.45 & 11.06 \\
0.940 & 172.06 & 10.11 & 11.83 \\
0.955 & 148.41 & 14.25 & 16.67 \\
\hline
\end{tabular}

Table 6: $\eta$ differential cross section for center of mass energy $=2750 \mathrm{MeV}$

\begin{tabular}{|c|c|c|c|}
\hline $\cos (\theta)$ & CRX $($ nb/str $)$ & statistical error & sys $s_{\text {err }}$ \\
\hline-0.902 & 17.10 & 8.47 & 11.27 \\
-0.726 & 2.46 & 0.69 & 0.92 \\
-0.445 & 0.21 & 0.31 & 0.41 \\
-0.171 & 1.57 & 0.30 & 0.40 \\
0.095 & 2.30 & 0.37 & 0.49 \\
0.307 & 3.40 & 0.45 & 0.60 \\
0.438 & 7.78 & 0.85 & 1.13 \\
0.542 & 15.11 & 1.20 & 1.60 \\
0.645 & 27.06 & 1.58 & 2.10 \\
0.745 & 53.82 & 2.29 & 3.05 \\
0.811 & 84.83 & 4.51 & 6.00 \\
0.851 & 114.27 & 5.29 & 7.04 \\
0.890 & 140.53 & 6.03 & 8.02 \\
0.920 & 155.85 & 9.28 & 12.34 \\
0.940 & 160.93 & 10.19 & 13.55 \\
0.955 & 180.17 & 15.58 & 20.72 \\
\hline
\end{tabular}

Table 7: $\eta$ differential cross section for center of mass energy $=2810 \mathrm{MeV}$ 


\begin{tabular}{|c|c|c|c|}
\hline $\cos (\theta)$ & CRX $($ nb/str $)$ & statistical error & sys $_{\text {err }}$ \\
\hline-0.902 & 8.27 & 2.66 & 2.69 \\
-0.716 & 1.45 & 0.53 & 0.54 \\
-0.448 & 1.16 & 0.29 & 0.29 \\
-0.156 & 1.27 & 0.25 & 0.25 \\
0.092 & 1.28 & 0.26 & 0.26 \\
0.306 & 2.44 & 0.33 & 0.33 \\
0.442 & 5.17 & 0.67 & 0.68 \\
0.545 & 9.17 & 0.88 & 0.89 \\
0.645 & 21.01 & 1.26 & 1.27 \\
0.744 & 48.30 & 1.92 & 1.94 \\
0.810 & 70.47 & 3.76 & 3.80 \\
0.851 & 104.14 & 4.54 & 4.59 \\
0.891 & 126.89 & 5.20 & 5.25 \\
0.920 & 144.98 & 7.97 & 8.05 \\
0.940 & 177.12 & 9.15 & 9.24 \\
0.955 & 169.17 & 13.36 & 13.49 \\
0.965 & 33.52 & 7.45 & 7.52 \\
\hline
\end{tabular}

Table 8: $\eta$ differential cross section for center of mass energy $=2875 \mathrm{MeV}$

\begin{tabular}{|c|c|c|c|}
\hline $\cos (\theta)$ & CRX $($ nb/str $)$ & statistical error & sys $_{\text {err }}$ \\
\hline-0.900 & 6.16 & 1.40 & 1.34 \\
-0.713 & 21.18 & 55.84 & 53.61 \\
-0.459 & 0.89 & 0.24 & 0.23 \\
-0.161 & 0.60 & 0.17 & 0.16 \\
0.082 & 0.75 & 0.22 & 0.21 \\
0.316 & 2.09 & 0.29 & 0.28 \\
0.442 & 3.67 & 0.56 & 0.54 \\
0.542 & 7.22 & 0.80 & 0.77 \\
0.648 & 18.32 & 1.22 & 1.17 \\
0.745 & 36.59 & 1.77 & 1.70 \\
0.811 & 62.08 & 3.48 & 3.34 \\
0.850 & 84.38 & 4.21 & 4.04 \\
0.891 & 110.93 & 4.88 & 4.68 \\
0.920 & 128.44 & 7.78 & 7.47 \\
0.940 & 146.98 & 8.62 & 8.28 \\
0.955 & 153.23 & 13.71 & 13.16 \\
0.965 & 83.69 & 10.66 & 10.23 \\
\hline
\end{tabular}

Table 9: $\eta$ differential cross section for center of mass energy $=2955 \mathrm{MeV}$ 


\begin{tabular}{|c|c|c|c|}
\hline $\cos (\theta)$ & CRX $($ nb/str $)$ & statistical error & sys $_{\text {err }}$ \\
\hline-0.903 & 5.42 & 1.05 & 1.03 \\
-0.717 & 1.27 & 0.40 & 0.39 \\
-0.487 & 0.80 & 0.22 & 0.22 \\
-0.179 & 0.24 & 0.10 & 0.10 \\
0.101 & 0.47 & 0.13 & 0.13 \\
0.292 & 0.88 & 0.18 & 0.18 \\
0.452 & 0.98 & 0.33 & 0.32 \\
0.549 & 4.93 & 0.55 & 0.54 \\
0.646 & 12.76 & 0.87 & 0.85 \\
0.746 & 29.11 & 1.31 & 1.28 \\
0.810 & 51.84 & 2.77 & 2.71 \\
0.851 & 78.20 & 3.45 & 3.38 \\
0.890 & 104.08 & 3.96 & 3.88 \\
0.920 & 130.19 & 6.39 & 6.26 \\
0.940 & 138.39 & 7.11 & 6.97 \\
0.955 & 172.49 & 11.15 & 10.93 \\
\hline
\end{tabular}

Table 10: $\eta$ differential cross section for center of mass energy $=3053 \mathrm{MeV}$

\begin{tabular}{|c|c|c|c|}
\hline $\cos (\theta)$ & CRX $($ nb/str $)$ & statistical error & sys err \\
\hline-0.892 & 5.40 & 1.58 & 1.53 \\
-0.713 & 1.48 & 0.53 & 0.51 \\
-0.478 & 0.84 & 0.29 & 0.28 \\
-0.199 & 0.07 & 0.08 & 0.08 \\
0.101 & 0.11 & 0.07 & 0.07 \\
0.326 & 0.35 & 0.13 & 0.13 \\
0.446 & 1.02 & 0.25 & 0.24 \\
0.550 & 2.83 & 0.44 & 0.43 \\
0.646 & 10.19 & 0.77 & 0.75 \\
0.746 & 22.96 & 1.16 & 1.13 \\
0.812 & 42.40 & 2.50 & 2.42 \\
0.851 & 63.52 & 3.05 & 2.96 \\
0.891 & 94.68 & 3.78 & 3.67 \\
0.920 & 125.47 & 6.29 & 6.10 \\
0.940 & 130.64 & 6.66 & 6.46 \\
0.955 & 134.87 & 10.45 & 10.14 \\
0.965 & 157.90 & 11.85 & 11.49 \\
0.975 & 7.78 & 3.54 & 3.43 \\
\hline
\end{tabular}

Table 11: $\eta$ differential cross section for center of mass energy $=3155 \mathrm{MeV}$ 


\begin{tabular}{|c|c|c|c|}
\hline $\cos (\theta)$ & CRX $($ nb/str $)$ & statistical error & sys err \\
\hline-0.896 & 3.89 & 1.11 & 1.01 \\
-0.726 & 0.88 & 0.33 & 0.30 \\
-0.476 & 0.32 & 0.13 & 0.12 \\
-0.175 & 0.03 & 0.05 & 0.05 \\
0.125 & 0.06 & 0.05 & 0.05 \\
0.303 & 0.15 & 0.06 & 0.05 \\
0.441 & 0.38 & 0.17 & 0.15 \\
0.548 & 1.75 & 0.29 & 0.26 \\
0.647 & 5.83 & 0.50 & 0.46 \\
0.747 & 16.09 & 0.84 & 0.76 \\
0.811 & 31.39 & 1.89 & 1.72 \\
0.851 & 52.89 & 2.36 & 2.15 \\
0.891 & 69.93 & 2.83 & 2.58 \\
0.920 & 107.78 & 4.96 & 4.51 \\
0.940 & 122.25 & 5.57 & 5.07 \\
0.955 & 140.99 & 8.88 & 8.08 \\
0.965 & 155.30 & 9.67 & 8.80 \\
0.975 & 45.04 & 6.43 & 5.85 \\
\hline
\end{tabular}

Table 12: $\eta$ differential cross section for center of mass energy $=3277 \mathrm{MeV}$ 


\section{Bibliography}

[1] V. Mathieu, J. Nys, C. Fernndez-Ramrez, A. Jackura, M. Mikhasenko, A. Pilloni, A.P. Szczepaniak, and G. Fox. On the and photoproduction beam asymmetry at high energies. Physics Letters B, 774:362 - 367, 2017.

[2] B. Krusche and C. Wilkin. Production of $\eta$ and $\eta^{\prime}$ mesons on nucleons and nuclei. Progress in Particle and Nuclear Physics, 80:4395, Jan 2015.

[3] M Dugger, JP Ball, P Collins, E Pasyuk, BG Ritchie, G Adams, P Ambrozewicz, E Anciant, M Anghinolfi, B Asavapibhop, et al. $\eta^{\prime}$ photoproduction on the proton for photon energies from 1.527 to 2.227 gev. Physical review letters, 96(6):062001, 2006.

[4] Volker Crede, A McVeigh, AV Anisovich, JCS Bacelar, R Bantes, O Bartholomy, D Bayadilov, R Beck, YA Beloglazov, R Castelijns, et al. Photoproduction of $\eta$ and $\eta$ 'mesons off protons. Physical Review C, 80(5):055202, 2009.

[5] F Huang, H Haberzettl, and K Nakayama. Combined analysis of $\eta^{\prime}$ production reactions: $\gamma n \rightarrow \eta^{\prime} n, n n \rightarrow n n \eta^{\prime}$, and $\pi n \rightarrow \eta^{\prime} n$. Physical Review C, 87(5):054004, 2013 .

[6] J Nys, V Mathieu, C Fernández-Ramírez, AN Blin, A Jackura, M Mikhasenko, A Pilloni, AP Szczepaniak, G Fox, J Ryckebusch, et al. Finite-energy sum rules in eta photoproduction off the nucleon. arXiv preprint arXiv:1611.04658, 2016.

[7] Joseph Mancell, IJ Douglas MacGregor, Mainz A2 Collaboration, et al. Eta photoproduction on the proton. In Journal of Physics: Conference Series, volume 381, page 012024. IOP Publishing, 2012.

[8] T. Hu, Z. Akbar, and V. Crede et. al. Photoproduction of mesons off the proton for $1.2<e<4.7$ gev using clas at jefferson laboratory. 2020 .

[9] M Williams, Z Krahn, D Applegate, M Bellis, Curtis A Meyer, KP Adhikari, M Anghinolfi, H Baghdasaryan, J Ball, M Battaglieri, et al. Differential cross sections for the reactions $\gamma p \rightarrow p \eta$ and $\gamma p \rightarrow p \eta^{\prime}$. Physical Review C, 80(4):045213, 2009.

[10] V. L. Kashevarov, M. Ostrick, and L. Tiator. Regge phenomenology in $\pi^{0}$ and $\eta$ photoproduction. Phys. Rev. C, 96:035207, Sep 2017.

[11] L. Tiator, M. Gorchtein, V. L. Kashevarov, K. Nikonov, M. Ostrick, M. Hadimehmedovi, R. Omerovi, H. Osmanovi, J. Stahov, and A. varc. Eta and etaprime photoproduction on the nucleon with the isobar model etamaid2018. The European Physical Journal A, 54(12), Dec 2018.

[12] Curtis A Meyer and Y Van Haarlem. Status of exotic-quantum-number mesons. Physical Review C, 82(2):025208, 2010.

[13] GlueX Collaboration et al. Mapping the spectrum of light quark mesons and gluonic excitations with linearly polarized photons. Presentation to PAC, 30, 2006. 
[14] GlueX Collaboration. https://halldweb.jlab.org/doc-private/DocDB/ ShowDocument?docid=2442, 2016.

[15] Volker Crede and CA Meyer. The experimental status of glueballs. Progress in Particle and Nuclear Physics, 63(1):74-116, 2009.

[16] GS Adams, T Adams, Z Bar-Yam, JM Bishop, VA Bodyagin, BB Brabson, DS Brown, NM Cason, SU Chung, RR Crittenden, et al. Observation of a new j pc=1-+ exotic state in the reaction $\pi^{-} p \rightarrow \pi^{+} \pi^{-} \pi^{-} p$ at $18 \mathrm{gev} / \mathrm{c}$. Physical Review Letters, 81(26):5760, 1998.

[17] EI Ivanov, DL Stienike, DI Ryabchikov, GS Adams, T Adams, Z Bar-Yam, JM Bishop, VA Bodyagin, DS Brown, NM Cason, et al. Observation of exotic meson production in the reaction $\pi^{-} p \rightarrow \eta^{\prime} \pi^{-} p$ at 18 gev/c. Physical review letters, 86(18):3977, 2001.

[18] Frank Nerling. Observation of a $j^{P C}=1^{-+}$exotic signal in the $\pi^{-} \pi^{0} \pi^{0}$ system diffractively produced at compass, and comparison to the charged decay mode. In EPJ Web of Conferences, volume 37, page 09025. EDP Sciences, 2012.

[19] Jozef J Dudek. The lightest hybrid meson supermultiplet in qcd. Physical Review D, 84(7):074023, 2011.

[20] C.A. Meyer and E.S. Swanson. Hybrid mesons. Progress in Particle and Nuclear Physics, 82:21 - 58, 2015.

[21] Isgur Nathan and Paton Jack. Flux-tube model for hadrons in qcd. Phys. Rev. D, 31:2910-2929, Jun 1985.

[22] Thomson Mark. Modern particle physics. Cambridge University Press, 2013.

[23] C. Patrignani et al. Review of Particle Physics. Chin. Phys., C40(10):100001, 2016.

[24] Eberhard Klempt and Alexander Zaitsev. Glueballs, hybrids, multiquarks: Experimental facts versus qcd inspired concepts. Physics Reports, 454(1):1-202, 2007.

[25] M. Guidal, J.-M. Laget, and M. Vanderhaeghen. Pion and kaon photoproduction at high energies: forward and intermediate angles. Nuclear Physics A, 627(4):645 - 678, 1997.

[26] Eero Byckling and K. Kajantie. Particle Kinematics. University of Jyvaskyla, Jyvaskyla, Finland, 1971.

[27] Donnachie and Sandy et al. Pomeron Physics and QCD. Cambridge University Press, 2002 .

[28] Yan Wang. Cebaf overview. 2010.

[29] Charles E. Reece. Erratum: Continuous wave superconducting radio frequency electron linac for nuclear physics research [phys. rev. accel. beams 19, 124801 (2016)]. Phys. Rev. Accel. Beams, 22:129901, Dec 2019.

[30] Charles E. Reece. Continuous wave superconducting radio frequency electron linac for nuclear physics research. Phys. Rev. Accel. Beams, 19:124801, Dec 2016. 
[31] Richard T. Jones. Intense beams of polarized and nearly monochromatic photons from coherent bremsstrahlung. Talk given at the summer 1997 Hall D Workshop., 1997.

[32] U. Timm. Coherent bremsstrahlung of electrons in crystals. Fortschritte der Physik, 17(12):765-808, 1969.

[33] A. W. Sáenz and H. Überall. Theory of Coherent Bremsstrahlung, pages 5-31. Springer Berlin Heidelberg, Berlin, Heidelberg, 1985.

[34] Brendan Pratt. Diamond Radiator Fabrication, Characterization and Performance for the GlueX Experiment. UConn Library Catalog, 2017.

[35] GlueX Collaboration. Halld/gluex technical construction report. 2014.

[36] Stuart Fegan. The broadband fixed-array tagging hodoscope for the coheren bremsstrahlung facility in hall d at jefferson lab. https://halldweb.jlab.org/ doc-private/DocDB/ShowDocument?docid=4082, 2019.

[37] GlueX Collaboration. The gluex beamline and detector. Nuclear Instruments and Methods in Physics Research Section A: Accelerators, Spectrometers, Detectors and Associated Equipment, 2020.

[38] A Somov, I Tolstukhin, S V Somov, and V V Berdnikov. Commissioning of the pair spectrometer of the GlueX experiment. Journal of Physics: Conference Series, 798:012175, jan 2017.

[39] E. Pooser, F. Barbosa, W. Boeglin, C. Hutton, M.M. Ito, M. Kamel, P. Khetarpal, A. LLodra, N. Sandoval, S. Taylor, and et al. The gluex start counter detector. Nuclear Instruments and Methods in Physics Research Section A: Accelerators, Spectrometers, Detectors and Associated Equipment, 927:330342, May 2019.

[40] S. Adhikari, A. Ali, M. Amaryan, and et. al. The gluex beamline and detector. 2020.

[41] Torbjrn Sjstrand, Stephen Mrenna, and Peter Skands. PYTHIA 6.4 physics and manual. Journal of High Energy Physics, 2006(05):026-026, may 2006.

[42] Marc Paterno and /Fermilab and. Calculating efficiencies and their uncertainties. 12 2004.

[43] F. Barbosa. Electronics overview. https://halldweb.jlab.org/doc-public/ DocDB/ShowDocument?docid=2515, 2014.

[44] G. Visser, D. Abbot, F. Barbosa, C. Cuevas, H. Dong, E. Jastrzembski, B. Moffit, and B. Raydo. A 72 channel 125 msps analog-to-digital converter module for drift chamber readout for the gluex detector. In IEEE Nuclear Science Symposuim Medical Imaging Conference, pages 777-781, 2010.

[45] Michael Justin Staib. Calibrations for charged particle tracking and measurements of photoproduction with the GlueX detector. Carnegie Mellon University, 2017. 
[46] T.D. Beattie, A.M. Foda, C.L. Henschel, S. Katsaganis, S.T. Krueger, G.J. Lolos, Z. Papandreou, E.L. Plummer, I.A. Semenova, A.Yu. Semenov, and et al. Construction and performance of the barrel electromagnetic calorimeter for the gluex experiment. Nuclear Instruments and Methods in Physics Research Section A: Accelerators, Spectrometers, Detectors and Associated Equipment, 896:2442, Jul 2018.

[47] M. Tanabashi et al. Particle data group. Phys. Rev. D 98, 030001 (2018) and 2019 update.

[48] Paul Mattione. https://halldweb.jlab.org/wiki/index.php/Gluex_ Analysis_Software, 2013.

[49] Alex A. https://halldweb.jlab.org/wiki-private/index.php/Fall_ 2018_Analysis_Launch, 2020.

[50] Paul Mattione. https://halldweb.jlab.org/wiki/index.php/Dselector, 2016.

[51] Laget J M. Rescattering in meson photoproduction from few body systems. Phys. Rev., C73:044003, 2006.

[52] Simon Taylor. Modelling of $\eta$ and $\eta^{\prime}$ cross section. 2015.

[53] S Agostinelli et al. Geant4a simulation toolkit. Nuclear Instruments and Methods in Physics Research Section A: Accelerators, Spectrometers, Detectors and Associated Equipment, 506(3):250 - 303, 2003.

[54] Paul Eugenio. https://halldweb.jlab.org/doc-public/DocDB/ ShowDocument?docid=11.

[55] Vincent Mathieu. http//cgl.soic.indiana.edu/jpac/EtaPhot.php\# EtaPhot.

[56] J. Nys, V. Mathieu, C. Fernández-Ramírez, A. N. Hiller Blin, A. Jackura, M. Mikhasenko, A. Pilloni, A. P. Szczepaniak, G. Fox, and J. Ryckebusch. Finiteenergy sum rules in eta photoproduction off a nucleon. Phys. Rev. D, 95:034014, Feb 2017.

[57] Byung-Geel Yu and Kook-Jin Kong. Photoproductions of $f_{1}(1285)$ and $\eta^{\prime}(958)$ from the analysis of clas data with the primakoff effect at high energies. Phys. Rev. D, 102:054019, Sep 2020. 


\section{MAHMOUD KAMEL}

$1992-1997$

$1998-2003$

$2006-2011$

$2011-2013$

$2013-2020$
B.Sc., Physics

Cairo University

Cairo, Egypt

M.Sc., Meteorology

Cairo University

Cairo, Egypt

M.Sc., Oceanography

Old Dominion University

Norfolk, Virginia

M.Sc., Physics

Old Dominion University

Norfolk, Virginia

Ph.D., Physics

Florida International University

Miami, Florida

\section{SELECTED PUBLICATIONS AND PRESENTATIONS}

1. The GlueX Collaboration, S. Adhikari et al. Measurement of the Beam Asymmetry in $\gamma p \rightarrow K^{+} \Sigma$ at $E_{\gamma}=8.5 \mathrm{GeV}$, Phys. Rev. C (2020) arXiv:2003.08038

2. The GlueX Collaboration, S. Adhikari et al. First measurement of near-threshold $J / \Psi$ exclusive photoproduction off the proton, Phys. Rev. Lett. 123, 072001 (2019)

3. Pooser et al The GlueX Start Counter Detector, Nuclear Instruments and Methods in Physics Research Section A: Accelerators, Spectrometers, Detectors and Associated Equipment, 927:330342, May 2019 